Merci d'utiliser le titre suivant lorsque vous citez ce document :

Jones, D. et A. Kwieciński (2010-10-01), « Mesures prises dans les économies émergentes face aux flambées des cours internationaux des produits agricoles de base ", Éditions OCDE, Paris.

http://dx.doi.org/10.1787/5km6c60xbfhk-fr

\title{
Mesures prises dans les économies émergentes face aux flambées des cours internationaux des produits agricoles de base
}

Darryl Jones

Andrzej Kwieciński

La version originale de ce document a été publiée comme suit :

Jones, D. and A. Kwieciński (2010-10-01), "Policy Responses in Emerging Economies to International Agricultural Commodity Price Surges", OECD Food, Agriculture and Fisheries Papers, No. 34, OECD Publishing, Paris. http://dx.doi.org/10.1787/5km6c61fv40w-en 


\section{TABLE DES MATIÈRES}

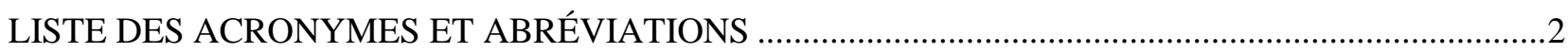

MESURES PRISES DANS LES ÉCONOMIES ÉMERGENTES FACE AUX FLAMBÉES DES COURS INTERNATIONAUX DES PRODUITS AGRICOLES DE BASE ..............................................

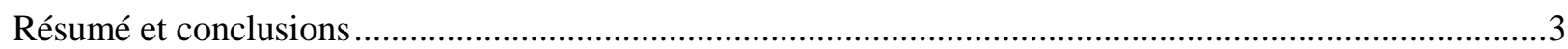

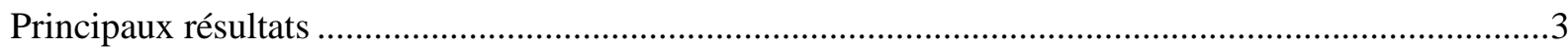

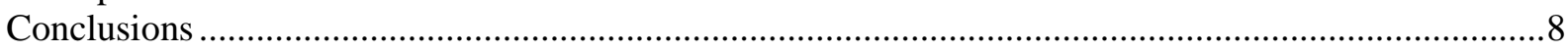

1. Examen et classification des mesures à court terme prises par les pouvoirs publics.........................10

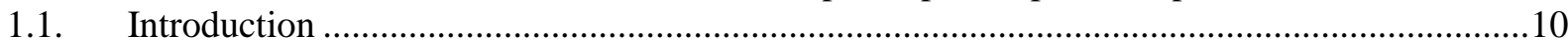

1.2. Mode de classification des mesures prises ..........................................................................

1.3. Estimation de l'impact budgétaire des mesures prises .............................................................15

1.4. Quelles ont été les mesures prises par les pouvoirs publics ?................................................16

1.5. Pourquoi les gouvernements ne prennent-ils pas tous des mesures identiques ? ........................30

2. Évaluation de l'impact sur le marché intérieur des mesures à court terme prises par les

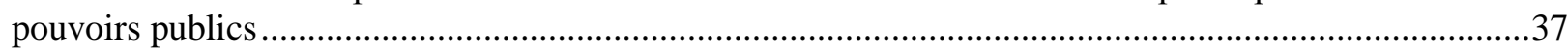

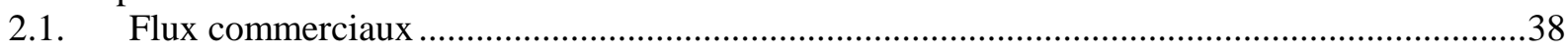

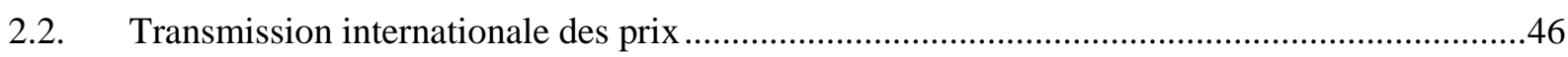

2.3. Prix à la consommation des produits alimentaires et inflation ..............................................53

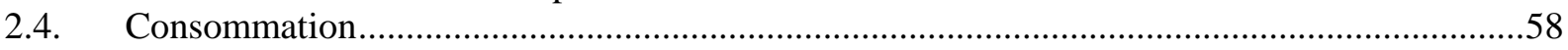

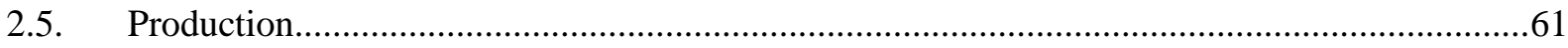

ANNEXE A. TABLEAUX DÉTAILLÉS DES MESURES À COURT TERME PRISES

(INCLUANT LE RENFORCEMENT DES MESURES DÉJÀ EN PLACE) …........................................68

ANNEXE B. ÉVOLUTIONS DES PRIX DES PRODUITS DE BASE DEPUIS 2006..............................98

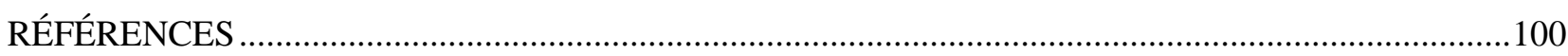




\section{LISTE DES ACRONYMES ET ABRÉVIATIONS}

\begin{tabular}{|c|c|}
\hline ARG & Argentine \\
\hline BRA & Brésil \\
\hline TEC & Tarif extérieur commun \\
\hline CHL & Chili \\
\hline $\mathrm{CHN}$ & Chine \\
\hline CAF & Coût, assurance et fret \\
\hline IPC & Indice des prix à la consommation \\
\hline $\mathrm{VC}$ & Variation compensatoire \\
\hline UE & Union européenne \\
\hline FAO & Organisation des Nations Unies pour l'alimentation et l'agriculture \\
\hline FAB & Franco à bord \\
\hline PIB & Produit intérieur brut \\
\hline RNB & Revenu national brut \\
\hline DI & Dollar international \\
\hline IDN & Indonésie \\
\hline IFPRI & Institut international de recherche sur les politiques alimentaires \\
\hline FMI & Fonds monétaire international \\
\hline IND & Inde \\
\hline UML & Unité monétaire locale \\
\hline PME & Prix minimum à l'exportation \\
\hline NPF & Nation la plus favorisée \\
\hline PSM & Prix de soutien minimum \\
\hline OCDE & Organisation de coopération et de développement économiques \\
\hline PPA & Parité de pouvoir d'achat \\
\hline ACR & Accords commerciaux régionaux \\
\hline RUS & Russie \\
\hline UKR & Ukraine \\
\hline USA & États-Unis d'Amérique \\
\hline TVA & Taxe sur la valeur ajoutée \\
\hline VNM & Vietnam \\
\hline PAM & Programme alimentaire mondial \\
\hline OMC & Organisation mondiale du commerce \\
\hline ZAF & Afrique du Sud \\
\hline
\end{tabular}

\section{Monnaies et taux de change}

$\begin{array}{ll}\text { ARS } & \text { Peso argentin } \\ \text { BRL } & \text { Réal brésilien } \\ \text { CLP } & \text { Peso chilien } \\ \text { CNY } & \text { Yuan/renminbi chinois } \\ \text { EUR } & \text { Euro } \\ \text { IDR } & \text { Roupie indonésienne } \\ \text { INR } & \text { Roupie indienne } \\ \text { RUB } & \text { Rouble russe } \\ \text { UAH } & \text { Hryvnia ukrainienne } \\ \text { USD } & \text { Dollar des États-Unis } \\ \text { VND } & \text { Dong vietnamien } \\ \text { ZAR } & \text { Rand sud-africain }\end{array}$




\section{MESURES PRISES DANS LES ÉCONOMIES ÉMERGENTES FACE AUX FLAMBÉES DES COURS INTERNATIONAUX DES PRODUITS AGRICOLES DE BASE}

\section{Résumé et conclusions}

Ce rapport porte sur dix grandes économies émergentes : l'Afrique du Sud, l'Argentine, le Brésil, le Chili, la Chine, l'Inde, l'Indonésie, la Russie, l'Ukraine et le Vietnam. Il a deux objectifs : (a) examiner et classer en différentes catégories les mesures à court terme prises dans ces pays face à la hausse des cours internationaux des produits de base au cours de la période 2006-08 et (b) analyser leurs répercussions sur les marchés intérieurs afin d'évaluer leur efficacité au regard des résultats visés. Il s'intéresse aux effets de l'action publique sur les flux commerciaux en provenance ou à destination de ces pays, mais il ne prend pas en considération les répercussions des réorientations des flux commerciaux sur les marchés internationaux, et notamment les pressions à la hausse des cours mondiaux des produits de base générées par les mesures de restriction des exportations. Ces répercussions sont examinées dans un autre document intitulé «Effets potentiels sur les marchés de certaines mesures envisageables dans les économies émergentes pour parer aux futures flambées des prix des produits agricoles de base » (Thompson et Tallard, 2010).

Pour répondre au premier de ces objectifs, un système de classification conforme à celui utilisé par les autres organismes internationaux qui ont étudié les mesures prises par les pouvoirs publics a été établi. Ce système répartit ces mesures en quatre grandes catégories : les interventions sur le marché destinées à limiter la hausse des prix alimentaires, les interventions sur le marché visant à maîtriser l'inflation, les mesures d'aide aux consommateurs au travers de filets de sécurité et celles de soutien aux producteurs. Toutes les mesures prises par les pouvoirs publics n'ont pas nécessairement des conséquences budgétaires clairement identifiables, mais le présent rapport n'en a pas moins pour particularité de s'efforcer d'estimer l'impact budgétaire de ces mesures pour évaluer l'importance relative de chacune d'elles dans un pays donné. Une seule et même méthode a été utilisée pour estimer les variations marginales des dépenses et des recettes budgétaires, à la hausse comme à la baisse. Les évolutions des flux commerciaux, la transmission des prix, l'inflation, la consommation et la production ont été autant d'éléments utilisés pour atteindre le second objectif. L'analyse porte essentiellement sur trois céréales (le blé, le maïs et le riz) et une oléagineuse (le soja). Bien souvent, un même produit de base était visé par plusieurs des mesures prises par les pouvoirs publics. Il n'est dès lors pas possible d'isoler les effets de chacune d'elles.

\section{Principaux résultats}

Huit des dix pays ont pris des mesures pour agir directement sur le prix de produits agricoles de base ou pour en accroître l'offre afin de limiter la hausse des prix des denrées alimentaires. Seuls l'Afrique du Sud et le Chili s'en sont abstenus. Le Brésil, la Chine, l'Inde, l'Indonésie, la Russie et le Vietnam on tous réduit ou supprimé les droits de douane sur certains produits de base, et ne sont dans certains cas pas revenus depuis sur ces dispositions. L'Argentine, la Chine, l'Inde, l'Indonésie, la Russie et le Vietnam ont instauré des taxes à l'exportation ou en ont accru le montant, ou bien ils ont réduit les incitations par les prix fournies pour encourager les exportations. Il faut toutefois souligner que, dans leur grande majorité, les interventions à court terme ont visé à renforcer les politiques déjà en place plutôt qu'à prendre de nouvelles mesures. Lorsque de nouvelles politiques ont été adoptées, elles ont le plus souvent consisté à imposer sous une forme ou une autre des mesures de restriction des exportations. Aussi peut-on dire que 
les mesures à court terme prises par les pouvoirs publics se sont dans la plupart des cas inscrites dans des cadres d'action à long terme et qu'elles étaient motivées par des objectifs à long terme tels que la sécurité alimentaire ou la stabilisation des revenus agricoles.

Pour ce qui est des conséquences budgétaires estimées, l'augmentation des recettes a été supérieure à celle des dépenses en Argentine, à hauteur de $0.1 \%$ de l'ensemble des recettes en 2008. Dans tous les autres pays, la progression des dépenses a été plus forte que celle des recettes. Révélateur d'un coût budgétaire, l'écart entre les dépenses et les recettes allait de $0.1 \%$ des recettes budgétaires au Chili jusqu'à $19 \%$ en Inde, mais se situait dans la plupart des cas dans une fourchette de $0.5 \%$ à $2.5 \%$. En 2008, d'un point de vue budgétaire, ce sont les filets de sécurité destinés à protéger les consommateurs qui ont pesé le plus lourd en Afrique du Sud, au Brésil et au Chili ; les interventions sur le marché en Argentine et au Vietnam; et les mesures de soutien aux producteurs en Chine, en Inde, en Indonésie, en Russie et en Ukraine.

Les différences d'un pays à l'autre du point de vue des types de mesures prises par les pouvoirs publics, tout comme de leur échelonnement dans le temps et de leur portée, reflètent leurs disparités sous l'angle du revenu national, du degré de pauvreté et de son incidence, de la part des dépenses consacrée à l'alimentation, de la contribution de l'agriculture au PIB et à l'emploi, du cadre d'action préexistant et des capacités budgétaires. Les mesures prises ont toutefois également été influencées par d'autres facteurs plus difficiles à quantifier tels que les systèmes politiques, les calendriers électoraux, les capacités institutionnelles existantes, les expériences passées, l'échelle de valeurs dominante ou la conception générale de l'action des pouvoirs publics. Par exemple, dans les quatre pays d'Asie, la place de l'alimentation dans la consommation des ménages et le poids de l'agriculture dans l'économie contribuent à expliquer qu'ils s'efforcent tout à la fois de protéger les consommateurs de la hausse des prix et de soutenir les producteurs en augmentant les subventions aux intrants et les prix d'achat minimums. En Afrique du Sud, au Brésil et au Chili, les pouvoirs publics disposent de plus grandes capacités budgétaires qui leur permettent de dédommager les consommateurs de la hausse des prix alimentaires, aussi ont-ils privilégié l'octroi d'un soutien direct aux consommateurs. Le Brésil et l'Argentine présentent certes de nombreuses similarités du point de vue des variables macro-économiques, ces deux pays ont réagi de manière très dissemblable, du fait en grande partie de différences concernant le cadre d'action en vigueur et les objectifs des pouvoirs publics.

Pour ce qui est des flux commerciaux, l'évaluation met l'accent sur l'effet des mesures de restriction des exportations. Il en ressort que les mesures prises en ce sens en Ukraine (contingents d'exportation), en Inde (interdiction des exportations, prix minimums et taxes à l'exportation) et en Chine (contingents, taxes et réduction des restitutions à l'exportation) ont sensiblement limité le volume des exportations des produits de base considérés. Les exportations annuelles de l'Argentine sont restées proches de leurs niveaux antérieurs, mais les mesures de restriction les ont maintenues en deçà de celui qu'elles auraient dû atteindre sous l'effet d'un marché mondial orienté à la hausse. Les mesures prises en Russie et au Vietnam n'ont pas nécessairement rejailli sur le volume global des exportations, mais elles en ont assurément modifié la composition et l'échelonnement dans le temps.

Des calculs de l'élasticité de la transmission des prix ont été effectués en vue de déterminer dans quelle mesure les dispositions prises ont permis d'isoler les marchés de gros intérieurs des fluctuations des cours internationaux des produits de base. L'appréciation en termes réels enregistrée par les monnaies nationales par rapport au dollar des États-Unis au cours de la période 2006-08 a atténué la hausse des cours mondiaux exprimée en monnaie locale dans chacun des dix pays étudiés à l'exception de l'Afrique du Sud, où le rand a perdu du terrain face au dollar des États-Unis. L'effet compensateur de l'appréciation de la monnaie en termes réels a été particulièrement marqué au Brésil, en Russie et en Ukraine. Ainsi, si le cours international du maïs exprimé en USD a progressé de 120\% en termes réels entre avril-juin 2006 et 
avril-juin 2008, son augmentation n'a été que de $60 \%$ environ en termes réels dans la monnaie locale de chacun de ces trois pays.

Après prise en compte des fluctuations des taux de change, il ressort de l'étude que ce sont les mesures prises en Inde et en Chine pour le riz, le blé et le maïs, et en Indonésie pour le riz et le soja qui ont le mieux isolé le marché intérieur pendant la période de hausse des cours internationaux (2006-08). Cette constatation découle d'une comparaison avec les degrés de transmission des prix pour ces mêmes produits dans d'autres pays ou pour d'autres produits dans le même pays (le soja dans le cas de l'Inde et de la Chine, et le blé dans celui de l'Indonésie), et avec le degré de transmission des prix sur les trois années précédentes (2003-06). En agissant sur l'échelonnement dans le temps des exportations et en augmentant les taxes à l'exportation, l'Argentine est parvenue à maintenir à un niveau relativement modéré pour un grand pays exportateur le degré de transmission à son marché intérieur des prix en vigueur sur le marché mondial. L'Ukraine a certes réussi à maîtriser les volumes exportés, mais elle n'a pas su limiter la transmission des prix de façon notable. Au Vietnam, les tentatives pour juguler la hausse des prix du riz n'ont eu aucun résultat. Si l'on compare les produits les produits, les degrés de transmission des prix ont généralement été plus élevés pour le soja que pour les trois céréales. Si l'on compare les pays, c'est en Afrique du Sud, au Brésil, au Chili et en Russie qu'ils ont atteint les plus hauts niveaux, tous produits confondus.

Dans les dix pays étudiés, les prix à la consommation des denrées alimentaires ont augmenté plus rapidement au cours de la période 2006/07-07/08 qu'en 2003/04-05/06, et c'est en Afrique du Sud et au Chili, deux économies relativement petites et ouvertes, qu'ils ont progressé le plus vite. Aussi l'inflation imputable aux prix alimentaires s'est-elle accrue dans chacun des dix pays entre ces deux périodes. En 2006/07-07/08, la hausse de l'inflation due à l'augmentation des prix alimentaires s'est établie entre $1.5 \%$ et $2.5 \%$ en Afrique du Sud, au Brésil, au Chili et en Indonésie, entre 4\% et 5\% en Argentine, en Chine, en Inde et en Russie, et entre $7.5 \%$ à $8.5 \%$ en Ukraine et au Vietnam, alors qu'elle n'était que de $0.6 \%$ dans la zone de l'OCDE. Les valeurs observées sont plus élevées dans les économies émergentes que dans les pays de l'OCDE, car les produits alimentaires pèsent plus lourd dans les indices de prix des premières que dans ceux des seconds. Surtout, dans la totalité des dix pays étudiés, les prix des denrées alimentaires ont augmenté plus vite que ceux des autres produits, en particulier au Chili, en Chine, en Ukraine et au Vietnam. Cependant, la totalité de la hausse des prix alimentaires ne peut être attribuée à l'augmentation des cours internationaux. Ces quatre pays ont connu des conditions météorologiques défavorables qui ont provoqué une augmentation conséquente des prix des fruits et des légumes produits sur place.

Pour mesurer l'impact sur la consommation de denrées alimentaires, la variation compensatoire (VC) liée aux évolutions des prix des aliments sur les périodes 2004-06 et 2007-09 a été estimée dans l'étude. La VC mesure la variation des revenus ou des dépenses monétaires nécessaire pour maintenir un niveau d'utilité constant après une modification des prix relatifs. Elle prend en considération l'évolution des prix des denrées alimentaires par rapport à ceux des autres produits, le poids de l'alimentation dans la consommation, mais aussi jusqu'à quel point les consommateurs peuvent aisément procéder à des substitutions entre la consommation de denrées alimentaires et celle d'autres produits de nature non alimentaire. Bien que l'Afrique du Sud et le Chili aient subi les plus fortes hausses des prix alimentaires, l'impact sur les consommateurs y a été relativement modeste si l'on prend ces autres facteurs en considération. L'étude montre que c'est probablement en Chine, en Inde, en Indonésie et au Vietnam qu'il a été le plus sensible. Or, ces quatre pays sont parmi ceux qui ont pris les mesures les plus vigoureuses pour intervenir sur leurs marchés intérieurs en vue de limiter le degré de transmission des prix, avec plus ou moins de réussite.

Si l'on utilise le PIB réel par habitant pour représenter le revenu des ménages, on s'aperçoit que la croissance économique a été suffisante dans tous les pays au cours de la période 2006-08 pour compenser largement la perte d'utilité subie par le consommateur moyen provoquée par l'évolution générale des prix 
relatifs au niveau national. En revanche, dans ce cas de figure, les consommateurs à faibles revenus sont durement touchés. Par ailleurs, le ralentissement de l'activité économique enregistré en 2008/09, venu s'ajouter à l'augmentation ininterrompue des prix des denrées alimentaires par rapport à ceux des autres produits, a probablement exercé sur l'ensemble des ménages une pression plus forte qu'entre la mi-2006 et la mi-2008, période où les cours mondiaux des produits de base ont connu leur hausse la plus rapide.

Il ressort de l'étude que les superficies consacrées à la culture des trois céréales étudiées et du soja ont en général augmenté dans les dix pays durant la période 2007-09, tout comme leurs volumes de production. Cette augmentation a été au moins égale, et bien souvent supérieure, à celle constatée aux États-Unis et dans le monde dans son ensemble. Dans les pays où la transmission des prix n'a pas été totale, comme la Chine, l'Inde et en Indonésie, la hausse des prix des achats publics et des subventions aux intrants a encouragé l'accroissement de la production. Les conditions météorologiques ont contribué à l'augmentation de la production de blé en Russie et en Ukraine, tandis qu'elles ont été défavorables à cette culture en Argentine et au Chili. Malgré des taxes à l'exportation plus élevées, les agriculteurs argentins délaissent le blé et le maïs au profit du soja car ce dernier a des coûts de production inférieurs et s'adapte mieux aux variations du climat, et les gouvernements successifs ont fait en sorte que les prix des céréales restent bas pour ne pas nuire aux consommateurs urbains. C'est tout l'inverse qui se produit en Chine, où les agriculteurs privilégient désormais les céréales au détriment des oléagineux pour profiter de la hausse du soutien accordé au titre de la production des premières.

Le tableau ci-après récapitule les principales mesures prises dans chacun des dix pays et leurs impacts marquants du point de vue des échanges, des prix, etc. Il présente également un résumé des principaux enseignements tirés dans chacun des cas. 
Tableau 1. Récapitulatif des mesures prises et de leurs impacts

\begin{tabular}{|c|c|c|c|}
\hline Pays & Principales mesures prises & Impacts & Résultats \\
\hline Argentine & $\begin{array}{l}\text { Augmentation des taxes à l'exportation et } \\
\text { maintien des restrictions quantitatives } \\
\text { applicables aux exportations de céréales et de } \\
\text { soja. Les recettes supplémentaires générées } \\
\text { par la hausse des taxes à l'exportation ont } \\
\text { permis de subventionner les transformateurs } \\
\text { afin de maintenir à un bas niveau les prix à la } \\
\text { consommation des produits alimentaires. }\end{array}$ & $\begin{array}{l}\text { Le marché intérieur des céréales a été isolé } \\
\text { des variations des cours mondiaux. Limitation } \\
\text { des flux commerciaux à leurs niveaux } \\
\text { antérieurs, probablement inférieurs à ceux } \\
\text { qu'ils auraient pu atteindre en l'absence des } \\
\text { taxes ou des restrictions applicables aux } \\
\text { exportations compte tenu de l'écart entre les } \\
\text { cours mondiaux et les prix intérieurs. }\end{array}$ & $\begin{array}{l}\text { Les mesures prises ont permis de maintenir les } \\
\text { prix intérieurs des céréales à un niveau } \\
\text { relativement bas, et ce à un très faible coût } \\
\text { budgétaire, protégeant ce faisant les } \\
\text { consommateurs mais au détriment des producteurs } \\
\text { qui doivent supporter une charge accrue. Elles ont } \\
\text { également entraîné une baisse de la production. } \\
\text { Les subventions aux transformateurs bénéficient à } \\
\text { tous les consommateurs et non pas uniquement } \\
\text { aux plus durement touchés. }\end{array}$ \\
\hline Brésil & $\begin{array}{l}\text { Augmentation des dépenses ciblées dans le } \\
\text { cadre du programme Bolsa Família; } \\
\text { ouverture de nouvelles lignes de crédit pour } \\
\text { les producteurs, y compris dans le cadre du } \\
\text { programme «Plus de nourriture »; certaines } \\
\text { réductions des droits de douane et autres taxes } \\
\text { à l'importation. }\end{array}$ & $\begin{array}{l}\text { Haut degré de transmission des prix au } \\
\text { marché intérieur. }\end{array}$ & $\begin{array}{l}\text { Les groupes de population les plus vulnérables, } \\
\text { qu'ils soient consommateurs ou producteurs, sont } \\
\text { protégés à un coût budgétaire plus élevé. Les } \\
\text { producteurs ont la possibilité de réagir à la hausse } \\
\text { des cours mondiaux en accroissant leur } \\
\text { production. }\end{array}$ \\
\hline Chili & $\begin{array}{l}\text { Augmentation exceptionnelle des allocations } \\
\text { versées aux consommateurs pauvres. }\end{array}$ & $\begin{array}{l}\text { Haut degré de transmission des prix. L'hiver } \\
\text { particulièrement rude dans le pays s'est } \\
\text { conjugué à la hausse des cours internationaux, } \\
\text { aboutissant à une augmentation relativement } \\
\text { importante des prix des produits alimentaires. }\end{array}$ & $\begin{array}{l}\text { Ce sont les consommateurs qui ont supporté le } \\
\text { poids de l'ajustement, partiellement atténué par } \\
\text { une aide ciblée en faveur des plus pauvres. Très } \\
\text { faible coût budgétaire. Les producteurs ont } \\
\text { bénéficié d'une hausse des prix des produits de } \\
\text { base. }\end{array}$ \\
\hline Chine & $\begin{array}{l}\text { Écoulement des stocks publics ; augmentation } \\
\text { des transferts aux consommateurs ; suspension } \\
\text { des remboursements de TVA à l'exportation; } \\
\text { instauration de taxes à l'exportation; } \\
\text { limitation du volume des exportations; } \\
\text { accroissement des subventions aux intrants; } \\
\text { encadrement des prix de gros et de détail. }\end{array}$ & $\begin{array}{l}\text { Le marché intérieur des céréales a été } \\
\text { partiellement isolé de la hausse des prix. Les } \\
\text { prix alimentaires ont augmenté sous l'effet de } \\
\text { facteurs internes - conditions climatiques et } \\
\text { épidémies. La production de céréales s'accroît } \\
\text { grâce à la hausse des subventions. }\end{array}$ & $\begin{array}{l}\text { Les consommateurs ont bénéficié de prix } \\
\text { relativement bas et stables, mais les producteurs } \\
\text { ont été taxés. Les producteurs ont été } \\
\text { partiellement dédommagés par une augmentation } \\
\text { des subventions aux intrants. Coût supporté par } \\
\text { les contribuables du fait de l'augmentation des } \\
\text { transferts aux consommateurs et du soutien aux } \\
\text { producteurs. }\end{array}$ \\
\hline Inde & $\begin{array}{l}\text { Instauration de mesures d'interdiction des } \\
\text { exportations, de prix minimums à } \\
\text { l'exportation, de taxes à l'exportation et } \\
\text { d'autres restrictions à l'encontre des } \\
\text { exportations; relèvement des prix d'achat } \\
\text { minimums mais maintien des prix } \\
\text { d'écoulement des stocks; augmentation des } \\
\text { subventions en faveur des engrais. }\end{array}$ & $\begin{array}{l}\text { Le marché intérieur des céréales a été isolé } \\
\text { des variations des cours mondiaux. La } \\
\text { production a été encouragée par la hausse du } \\
\text { soutien au titre de la production et des } \\
\text { intrants. Les stocks se sont accumulés. }\end{array}$ & $\begin{array}{l}\text { Les consommateurs ont bénéficié de prix } \\
\text { relativement bas et constants. Les producteurs ont } \\
\text { supporté une taxe sous la forme de prix inférieurs } \\
\text { aux cours internationaux mais ils ont bénéficié } \\
\text { d'un soutien au travers de subventions aux } \\
\text { intrants et de prix d'achat plus élevés fixés par les } \\
\text { autorités administratives. Les contribuables ont } \\
\text { supporté une lourde charge du fait d'une hausse } \\
\text { marquée des dépenses publiques, équivalente à } \\
19 \% \text { des recettes budgétaires. }\end{array}$ \\
\hline Indonésie & $\begin{array}{l}\text { Écoulement des stocks ; réduction des droits } \\
\text { de douane à l'importation; augmentation de la } \\
\text { distribution de riz et d'huile de friture } \\
\text { subventionnés; augmentation des prix de base } \\
\text { à l'exportation et de la taxe sur les } \\
\text { exportations d'huile de palme brute; } \\
\text { relèvement des prix d'achat de référence et } \\
\text { des subventions en faveur des engrais. }\end{array}$ & $\begin{array}{l}\text { Les marchés intérieurs du riz et du soja ont été } \\
\text { isolés de la hausse des cours mondiaux. } \\
\text { L'accroissement de la production a été stimulé } \\
\text { par les subventions accordées au titre de la } \\
\text { production et des intrants. }\end{array}$ & $\begin{array}{l}\text { Les politiques commerciales ont bénéficié aux } \\
\text { consommateurs, mais elles ont taxé les } \\
\text { producteurs. Les producteurs en ont été } \\
\text { partiellement dédommagés par un relèvement des } \\
\text { prix d'achat de référence et des subventions en } \\
\text { faveur des engrais. Les coûts ont principalement } \\
\text { été supportés par les contribuables du fait de la } \\
\text { hausse des dépenses de subvention des denrées } \\
\text { alimentaires et de leur production. }\end{array}$ \\
\hline Russie & $\begin{array}{l}\text { Déblocage des stocks publics ; application de } \\
\text { taxes sur les exportations de blé et d'orge ; } \\
\text { diminution des droits de douane à } \\
\text { l'importation d'un large éventail de produits } \\
\text { alimentaires; mesures de contrôle des prix des } \\
\text { produits alimentaires de première nécessité ; } \\
\text { augmentation des prix d'intervention pour } \\
\text { reconstituer les stocks. }\end{array}$ & $\begin{array}{l}\text { Effet sur l'échelonnement dans le temps des } \\
\text { exportations plutôt que sur le volume global } \\
\text { de celles-ci. Faible impact sur la transmission } \\
\text { des prix. Fort accroissement de la production } \\
\text { à la suite de la hausse des prix et de conditions } \\
\text { météorologiques favorables. }\end{array}$ & $\begin{array}{l}\text { Les consommateurs n'ont pas été protégés des } \\
\text { hausses de prix. Les producteurs ont bénéficié des } \\
\text { évolutions survenues sur les marchés. }\end{array}$ \\
\hline $\begin{array}{l}\text { Afrique du } \\
\text { Sud }\end{array}$ & $\begin{array}{l}\text { Forte hausse des dépenses au titre des aides } \\
\text { sociales ; soutien accru aux petits producteurs. }\end{array}$ & $\begin{array}{l}\text { Haut degré de transmission des prix et } \\
\text { augmentation relativement forte des prix des } \\
\text { produits alimentaires. }\end{array}$ & $\begin{array}{l}\text { Le coût a été supporté par les contribuables et par } \\
\text { les consommateurs ne bénéficiant pas d'aides } \\
\text { sociales. Les consommateurs pauvres ont quant à } \\
\text { eux bénéficié d'une augmentation des aides } \\
\text { sociales. }\end{array}$ \\
\hline Ukraine & $\begin{array}{l}\text { Instauration de contingents d'exportation de } \\
\text { céréales et plafonnement de la hausse des prix } \\
\text { à la consommation ; augmentation des prix } \\
\text { d'achat minimums. }\end{array}$ & $\begin{array}{l}\text { L'action des pouvoirs publics a limité les } \\
\text { exportations, mais elle n'a pas isolé le marché } \\
\text { intérieur de la hausse des cours mondiaux. }\end{array}$ & $\begin{array}{l}\text { Les consommateurs ont dû faire face à la hausse } \\
\text { des prix, tandis que les producteurs n'ont pas été } \\
\text { autorisés à tirer un profit maximum de la hausse } \\
\text { des cours mondiaux. Le coût budgétaire a été } \\
\text { limité. }\end{array}$ \\
\hline Vietnam & $\begin{array}{l}\text { Régulation des exportations de riz en volume } \\
\text { et en valeur ; réduction des droits de douane à } \\
\text { l'importation d'un large éventail de produits. }\end{array}$ & $\begin{array}{l}\text { L'action des pouvoirs publics n'a pas permis } \\
\text { d'isoler le marché intérieur du riz de la hausse } \\
\text { des cours mondiaux; augmentation } \\
\text { relativement forte des prix alimentaires, en } \\
\text { partie imputable aux prix élevés du riz. }\end{array}$ & $\begin{array}{l}\text { Les consommateurs ont supporté le coût de la } \\
\text { hausse des prix. La croissance rapide du PIB les a } \\
\text { aidés à faire face à l'augmentation des prix } \\
\text { alimentaires. Les producteurs n'ont pas été } \\
\text { autorisés à tirer un profit maximum de la hausse } \\
\text { des cours mondiaux. }\end{array}$ \\
\hline
\end{tabular}




\section{Conclusions}

Conjuguées aux difficultés rencontrées par certains pays pour limiter l'augmentation des prix alimentaires sur leur marché intérieur, la durée relativement courte de la flambée des prix internationaux des produits de base et les hausses saisonnières des prix des fruits et légumes survenues dans certains pays sous l'effet d'événements météorologiques marqués militent en faveur de la mise en place de filets de sécurité ciblés dans l'optique d'apporter une réponse pérenne à l'instabilité des prix alimentaires. Du fait de leur souplesse, ces mécanismes permettent en effet de faire face aux conséquences des hausses des prix pour les consommateurs sans perturber les marchés, et en particulier sans fausser les signaux transmis par les prix aux agriculteurs. Une fois le filet de sécurité en place, les transferts peuvent être accrus lorsque les prix montent, et réduits lorsque les prix baissent. La «prime» exceptionnelle versée aux bénéficiaires d'une allocation au Chili en est un bon exemple. En Afrique du Sud et au Brésil, les pouvoirs publics ont également ciblé les personnes les plus durement touchées, mais ont pour ce faire relevé le niveau de l'allocation de base. Il est dans ce cas bien plus difficile de revenir en arrière par la suite, et la charge pèse plus longtemps sur les finances publiques. Il ressort de l'étude qu'il convient de se pencher sur les coûts à long terme de ce type de programmes. Les gouvernements indiens successifs n'ayant pas augmenté depuis 2001 les prix de vente subventionnés appliqués aux personnes défavorisées, le coût budgétaire du système de distribution publique ciblée (Targeted Public Distribution System - TPDS) s'est rapidement alourdi.

L'étude met en évidence le dilemme lié à la panoplie «optimale» d'instruments d'action souvent préconisée par les organismes internationaux, qui consiste à permettre l'augmentation des prix alimentaires de sorte que les producteurs perçoivent les signaux du marché, à combattre l'inflation générale en relevant les taux d'intérêt, etc., et à mettre en place des filets de sécurité ciblés pour les pauvres les plus touchés. Le dilemme tient au fait que les deux premières mesures accroissent le coût de la troisième. Les transferts nécessaires pour dédommager les consommateurs sont en effet d'autant plus élevés que la hausse des prix des denrées alimentaires est importante par rapport à celle des autres produits, non alimentaires. Cela ne signifie pas que cette combinaison de mesures soit inadaptée, mais simplement que les mesures prises par les pouvoirs publics sont interdépendantes, et que plus les premières s'avèrent efficaces, plus le coût budgétaire de la troisième risque d'être élevé.

Outre les filets de sécurité destinés aux pauvres, l'étude révélait à quel point l'augmentation des revenus dans les économies émergentes joue un rôle essentiel en ce qui concerne la compensation des pertes d'utilité subies par les consommateurs du fait de la hausse relative des prix alimentaires. Aussi les politiques susceptibles de stimuler la croissance économique d'ensemble et de garantir que les avantages accordés aux ménages s'inscrive dans une panoplie d'instruments d'action permettant de se préparer efficacement à faire face aux futures flambées des prix.

L'intervention directe des pouvoirs publics, et notamment l'instauration de mesures de restriction des exportations, n'a pas toujours permis de réduire à néant les pressions exercées sur les prix intérieurs. L'expérience du marché des céréales en Ukraine et du marché du riz au Vietnam sert de mise en garde quant aux difficultés que soulève le recours aux mesures commerciales pour maitriser les prix intérieurs. Ces mesures se sont révélées plus efficaces dans les pays qui avaient déjà mis en place un système bien développé d'interventions sur le marché intérieur du produit de base considéré. Cependant, là où elles se sont avérées efficaces, les interventions directes n'ont pas été sans coût. En Argentine, les pouvoirs publics ont su maintenir les prix à la consommation à un niveau inférieur à celui qu'ils auraient atteint en l'absence d'intervention de leur part, mais celle-ci a en partie vidé de leur substance les incitations destinées aux producteurs de blé. La Chine, l'Inde et l'Indonésie ont elles aussi réussi à contenir la hausse des prix, mais pour dédommager les producteurs et stimuler la production, elles ont accru leur soutien aux producteurs lié aux intrants. 
À terme, les signaux transmis aux agriculteurs par ces prix en baisse pourraient bien exacerber les problèmes auxquels les pouvoirs publics tentaient initialement de trouver une solution. Il risque de s'ensuivre une baisse de la production intérieure, entraînant dans certains cas une augmentation des importations des produits de base considérés comme des denrées alimentaires de première nécessité, et ce à des prix supérieurs aux prix réglementés en vigueur sur les marchés intérieurs. En outre, les fréquentes ingérences, parfois opaques, des pouvoirs publics sur les marchés peuvent également avoir pour effet de saper la confiance des participants dans le bon fonctionnement des marchés. Les mesures de contrôle des prix et de restriction des exportations risquent de ne pas contribuer à la création d'un marché concurrentiel capable de supporter les crises soudaines.

Plusieurs pays ont réagi en relevant les prix minimums ou les prix d'intervention afin de reconstituer les stocks publics qui avaient été réduits pour stabiliser les prix intérieurs. Ces mesures ont souvent été annoncées et mises en œuvre juste avant que les cours internationaux des produits de base ne recommencent à baisser, à la mi-2008. Aussi les stocks publics ont-ils considérablement augmenté. Les stocks de blé de l'Inde ont ainsi atteint en juin 2010 un niveau quasiment sans précédent de 35 millions de tonnes, chiffre à peine inférieur à la production annuelle de la France, qui est le cinquième producteur mondial. Bien que ces stocks soient destinés à atténuer l'impact des pénuries temporaires de denrées alimentaires et qu'ils puissent s'avérer utiles, en particulier pour les grands pays qui disposent de capacités limitées de manutention aux frontières et dans les installations portuaires, l'achat et l'entreposage de telles quantités de produits imposent inévitablement d'importants coûts budgétaires. Ils risquent en outre de déstabiliser les marchés. L'existence de stocks aussi importants pourrait provoquer une chute des prix l'année suivante. Ils risquent par ailleurs de déstabiliser les cours mondiaux si les excédents sont exportés moyennant des subventions ou par une entreprise de commerce d'État à des prix inférieurs à leur coût d'achat.

Face à la crise, certains pays ont accru les subventions aux intrants dont bénéficient les producteurs agricoles. Sous réserve qu'elles s'inscrivent dans un train de mesures adapté et qu'elles soient complétées par des conseils pertinents, ces subventions peuvent favoriser la production de certains produits. L'expérience des pays de l'OCDE montre toutefois que leur impact positif sur le revenu des agriculteurs peut s'avérer modeste par rapport à leur coût budgétaire. Les subventions aux intrants agricoles peuvent également être étroitement liées aux atteintes à l'environnement, et en particulier à la pollution de l'eau. 


\section{Examen et classification des mesures à court terme prises par les pouvoirs publics}

\subsection{Introduction}

Au cours de la période de deux ans allant de la mi-2006 à la mi-2008, les cours internationaux des céréales, des oléagineux et des produits laitiers ont spectaculairement augmenté, et ils ont plus que doublé dans bien des cas. Tous les prix ont certes diminué par rapport aux pics précédemment atteints, mais ils demeurent pour bon nombre de produits de base bien supérieurs à leur niveau au milieu des années 2000. Aussi les pouvoirs publics se trouvent-ils confrontés à un certain nombre de problèmes, tout particulièrement dans les pays en développement. Au niveau macroéconomique, de nombreux pays en développement ont dû faire face à une nette augmentation du coût de leurs importation, d'où une dégradation de leur balance des paiements et des pressions sur les réserves en devises qui n'ont pas été sans conséquences sur la croissance et le développement (FMI, 2008a; FMI, 2008b). Au niveau microéconomique, les ménages pauvres des pays importateurs comme des pays exportateurs de produits alimentaires ont été les plus durement touchés compte tenu de la place importante qu'occupe l'alimentation dans leurs dépenses totales et des limitations auxquels ils se heurtent du fait de leurs bas revenus et de leur faible dotation en capital (PAM, 2009; Zezza et al., 2009). Il n'en résulte pas seulement une consommation alimentaire réduite, avec la malnutrition qui s'ensuit, mais aussi une diminution des dépenses et des investissements non alimentaires, prenant par exemple la forme d'une baisse de la fréquentation des établissements scolaires ou d'un moindre recours aux soins de santé (Banque mondiale, 2008a ; Ligon, 2008). En revanche, la hausse des cours mondiaux des produits alimentaires offre aux producteurs agricoles la possibilité de bénéficier de revenus accrus (James et al., 2008 ; Thapa et al., 2009).

Les gouvernements des pays de l'OCDE et des pays non membres de l'Organisation ont réagi à cette hausse marquée des prix par un large éventail d'initiatives, dont l'adoption de mesures de réduction des droits de douane à l'importation, de contrôle des prix, de restriction des exportations, de déblocage des stocks, ou encore la mise en place de programmes alimentaires. Des efforts considérables ont été déployés pour suivre les différents types de mesures adoptées. De vastes enquêtes sur les initiatives prises par les pouvoirs publics ont couvert un grand nombre de pays en développement (Demeke et al., 2008; FAO, 2009a; Viatte et al., 2009). D'ampleur plus restreinte, le rapport intitulé Politiques agricoles des économies émergentes 2009 : Suivi et évaluation présente, pour chacun des sept pays couverts, un encadré spécial sur la hausse des prix alimentaires résumant brièvement les mesures prises par les pouvoirs publics pour y faire face et la transmission au marché intérieur du renchérissement des cours internationaux des produits de base (OCDE, 2009a). Le Forum mondial sur l'Agriculture organisé par l'OCDE en 2009 «Perspectives agricoles : préparer l'avenir »- a consacré une session aux réponses à court terme à la hausse et à la volatilité des prix des produits alimentaires, donnant lieu à des exposés sur la Chine, le Brésil, l'Inde et l'Afrique du Sud (OCDE, 2009b).

En comparaison, relativement peu de travaux ont visé à évaluer l'efficacité des mesures prises par les pouvoirs publics. «Il faut renforcer le suivi et l'évaluation de l'efficacité par rapport aux coûts des mesures adoptées par les gouvernements » (FAO, 2009a). Dans le même ordre d'idées, un consultant a rédigé pour le compte de l'OCDE un rapport sur les conséquences sur le développement qui résultent du prix élevé des produits alimentaires. Il parvient à la conclusion que les futures «priorités en matière de recherche seront notamment de mieux comprendre quels en sont les impacts sur la faim et la pauvreté, eu égard à l'expérience effectivement acquise et à la nature des mesures prises par les pouvoirs publics, jusqu'à quel point ces mesures favorisent la stabilité ou l'instabilité des marchés intérieurs et internationaux, et si les initiatives de développement agricole engagées par la suite vont bien dans le bon sens » (Abbott, 2009).

Cette étude vise à évaluer l'efficacité des mesures prises par les pouvoirs publics au regard de leurs objectifs déclarés. Une telle évaluation présente un grand intérêt car beaucoup d'analystes s'attendent à une 
plus grande volatilité des prix à l'avenir du fait de la tendance générale à la réduction des stocks et du lien toujours plus étroit entre les prix des récoltes et ceux de l'énergie (OCDE, 2009b; OCDE, 2009c). Comprendre quelles sont les mesures qui ont donné de bons résultats durant la crise de 2006-08 et quelles sont celles qui se sont au contraire soldées par un échec contribuera utilement à déterminer les options à privilégier face aux éventuelles hausses de prix futures. Les mesures retenues ont parfois manqué de cohérence, certaines faisant appel à des mécanismes allant à l'encontre des objectifs de certaines autres. Certaines de ces mesures ont en outre eu des effets négatifs sur les échanges internationaux et n'ont fait qu'aggraver les pressions à la hausse des prix. Ces répercussions sont examinées dans une autre étude entreprise dans le cadre d'un projet plus large de l'OCDE portant sur les mutations structurelles des marchés des produits agricoles de base, projet auquel le présent rapport apporte également une contribution.

Compte tenu du peu de temps qui s'est écoulé depuis que pouvoirs publics ont pris ces mesures, et eu égard aux données disponibles, ce rapport porte essentiellement sur les initiatives mises en œuvre pour atteindre des objectifs à court terme, c'est-à-dire sur une durée d'un ou deux ans tout au plus. Il s'intéresse principalement, mais pas exclusivement, à celles de ces mesures qui visent à réduire l'impact exercé sur le marché intérieur par la hausse des cours internationaux. À très court terme (moins d'un an), les responsables de l'élaboration des politiques ne peuvent guère agir sur la production intérieure de produits alimentaires si les exploitants ont déjà pris leurs décisions quant au choix des cultures et à l'utilisation d'intrants pour la récolte à venir. L'évaluation de l'efficacité des mesures visant à atteindre des objectifs à plus long terme, qu'il s'agisse par exemple de renforcer le financement des activités de recherche et de développement afin d'accroître la productivité agricole, ou encore de mettre en place de nouveaux filets de sécurité pour aider les plus vulnérables, n'entre par contre pas dans le champ du présent rapport.

Seules sont prises en considération les mesures mises en œuvre au niveau national. Certaines initiatives ont été prises au plan infranational, comme par exemple les programmes de transfert de revenus à l'échelle d'une province ou encore les subventions à l'industrie meunière locale, mais il n'en sera pas tenu compte. Ne sont pas non plus examinées les mesures adoptées à la suite de la crise financière mondiale et du ralentissement économique qu'elle a entraîné dans le courant du second semestre 2008. Il en est par exemple ainsi de l'extension des possibilités de bénéficier de crédits bonifiés accordées aux exploitants, aux transformateurs, aux négociants, etc., afin de compenser le durcissement des conditions d'accès aux prêts du secteur privé.

Le reste de cette section examine les mesures prises par les pouvoirs publics dans dix grandes économies émergentes : les sept déjà étudiées dans le rapport intitulé Politiques agricoles des économies émergentes 2009: Suivi et évaluation, à savoir le Brésil, le Chili, la Chine, l'Inde, la Russie, l'Afrique du Sud et l'Ukraine, auxquelles viennent s'ajouter l'Argentine, l'Indonésie et le Vietnam. La section 2 évalue l'impact sur le marché intérieur des mesures à court terme prises par les pouvoirs publics et jusqu'à quel point elles sont parvenues à atteindre leurs objectifs. Divers indicateurs sont utilisés pour examiner les évolutions qui affectent les flux commerciaux, la transmission des prix, l'inflation, la consommation et la production.

\section{2. $\quad$ Mode de classification des mesures prises}

Pour chacun des dix pays, un tableau détaillé décrivant chacune des mesures prises par les pouvoirs publics, le(s) produit(s) de base concerné(s), la date où elle a commencé à être appliquée et celle où il y a été mis fin (s'il y a lieu), ses conséquences budgétaires (le cas échéant), de même que ses objectifs déclarés, est présenté à l'annexe A. Ces tableaux font partie intégrante du rapport et contiennent la plupart des informations détaillées concernant les mesures prises par les pouvoirs publics. Ils sont regroupés en annexe afin de mieux structurer le rapport. 
Un système de classification permettant de répartir les diverses mesures prises par les pouvoirs publics en 18 catégories distinctes a été utilisé pour mettre en évidences les différences selon les pays. Les mesures en question sont d'abord classées dans l'une de ces catégories, puis la liste en est dressée au sein de chacune des catégories en fonction du produit de base concerné et selon la date du début de leur mise en œuvre. Ce système de classification répertorie les mesures prises par les pouvoirs publics en fonction de leurs objectifs et de leur mode de fonctionnement. Pour ce qui est de leurs objectifs, les mesures sont divisées en quatre grandes catégories (tableau 1.1), Selon qu'elles visent à : (a) limiter les hausses des prix des produits alimentaires sur le marché intérieur; (b) maîtriser la hausse du niveau général des prix ; (c) aider les consommateurs confrontés au renchérissement des denrées alimentaires ; et (d) soutenir les producteurs pour accroître la production alimentaire. Les grandes catégories (a), (c) et (d) sont similaires à celles utilisées dans les études réalisées à la demande de la FAO (Demeke et al., 2008 ; Viatte et al., 2009), effectuées par la Banque mondiale (Banque mondiale, 2008b) ou menées à bien dans des instituts de recherche tels que l'IFPRI (Benson et al., 2008). Elles correspondent en outre aux trois grands volets du Programme d'intervention en réponse à la crise alimentaire mondiale mis en œuvre par la Banque mondiale (Banque mondiale, 2008b). Les mesures classées dans chacune de ces grandes catégories sont ensuite réparties dans des catégories plus fines mettant en lumière les différences dans leur mode de fonctionnement ou de mise en œuvre.

Les différentes catégories d'interventions sur le marché indiquent sur quels «points » du marché porte l'action des pouvoirs publics. Une distinction est établie entre les mesures spécifiquement axées sur le marché de l'alimentation (M1-M6) et celles de nature macroéconomique qui affectent l'ensemble de l'économie (I1). Les premières s'attachent à modifier le prix relatif des produits alimentaires au sein d'une économie, alors que les secondes ont un impact sur l'ensemble des prix. 
Tableau 1.1. Mode de classification des mesures à court terme prises par les pouvoirs publics

\begin{tabular}{|c|c|c|c|}
\hline $\begin{array}{l}\text { Grands types } \\
\text { de mesures }\end{array}$ & Catégories & Étiquette & Exemples \\
\hline \multirow{9}{*}{$\begin{array}{l}\text { Interventions } \\
\text { sur le marché } \\
\text { pour limiter la } \\
\text { hausse des prix } \\
\text { alimentaires }\end{array}$} & $\begin{array}{l}\text { Agit directement sur le prix des produits de } \\
\text { base - importations }\end{array}$ & M1 & $\begin{array}{l}\text { Réduction/suppression des tarifs et des } \\
\text { droits de douane }\end{array}$ \\
\hline & $\begin{array}{l}\text { Agit directement sur le prix des produits de } \\
\text { base - exportations }\end{array}$ & M2 & $\begin{array}{l}\text { Instauration/augmentation des taxes à } \\
\text { l'exportation }\end{array}$ \\
\hline & $\begin{array}{l}\text { Agit directement sur le prix des produits de } \\
\text { base - mesure de nature budgétaire }\end{array}$ & M3 & Réduction de la TVA, subventions aux prix \\
\hline & $\begin{array}{l}\text { Agit directement sur le prix des produits de } \\
\text { base-mesure non budgétaire }\end{array}$ & M4 & Mesures de contrôle administratif des prix \\
\hline & $\begin{array}{l}\text { Accroître/maintenir l'offre intérieure de produits } \\
\text { de base - importations }\end{array}$ & M5 & $\begin{array}{l}\text { Extension des autorisations sanitaires et } \\
\text { phytosanitaires, assouplissement du } \\
\text { système de licences d'importation }\end{array}$ \\
\hline & $\begin{array}{l}\text { Accroître/maintenir l'offre intérieure de produits } \\
\text { de base - exportations }\end{array}$ & M6 & $\begin{array}{l}\text { Augmentation des prix minimums à } \\
\text { l'exportation, instauration de contingents } \\
\text { d'exportation }\end{array}$ \\
\hline & $\begin{array}{l}\text { Accroître/maintenir l'offre intérieure de produits } \\
\text { de base - stocks }\end{array}$ & M7 & $\begin{array}{l}\text { Déblocage des stocks de réserves } \\
\text { alimentaires, application de sanctions en } \\
\text { cas d'accaparement }\end{array}$ \\
\hline & $\begin{array}{l}\text { Diminuer la demande non alimentaire du produit } \\
\text { de base considéré }\end{array}$ & M8 & $\begin{array}{l}\text { Restrictions aux transactions privées, } \\
\text { réajustements de la politique en matière de } \\
\text { biocarburants }\end{array}$ \\
\hline & Améliorer le fonctionnement du marché & M9 & $\begin{array}{l}\text { Plus grande transparence des prix, } \\
\text { création/interdiction des marchés à terme }\end{array}$ \\
\hline $\begin{array}{l}\text { Interventions } \\
\text { sur le marché } \\
\text { pour maîtriser } \\
\text { l'inflation } \\
\end{array}$ & Impact sur l'ensemble des prix & I1 & $\begin{array}{l}\text { Politique monétaire d'augmentation des } \\
\text { taux d'intérêt officiels, interventions visant } \\
\text { à apprécier le taux de change }\end{array}$ \\
\hline \multirow{2}{*}{$\begin{array}{l}\text { Filets de } \\
\text { sécurité pour } \\
\text { les } \\
\text { consommateurs }\end{array}$} & Aide pécuniaire & $\mathrm{C} 1$ & $\begin{array}{l}\text { Augmentation des transferts en espèces, } \\
\text { distribution de bons ou tickets } \\
\text { d'alimentation }\end{array}$ \\
\hline & Aide alimentaire & $\mathrm{C} 2$ & $\begin{array}{l}\text { Programmes de transferts de produits } \\
\text { alimentaires et de restauration scolaire }\end{array}$ \\
\hline \multirow{5}{*}{$\begin{array}{l}\text { Mesures axées } \\
\text { sur la } \\
\text { production }\end{array}$} & $\begin{array}{l}\text { Transferts au titre de la production de produits de } \\
\text { base }\end{array}$ & $\mathrm{P} 1$ & $\begin{array}{l}\text { Relèvement des prix à la production } \\
\text { garantis }\end{array}$ \\
\hline & Transferts au titre des intrants variables & $\mathrm{P} 2$ & $\begin{array}{l}\text { Augmentation des subventions en faveur } \\
\text { des engrais, développer les programmes de } \\
\text { distribution de semences }\end{array}$ \\
\hline & Transferts au titre de la formation de capital fixe & P3 & $\begin{array}{l}\text { Développement des possibilités d'obtention } \\
\text { de crédits bonifiés }\end{array}$ \\
\hline & $\begin{array}{l}\text { Transferts au titre des services utilisés sur } \\
\text { l'exploitation }\end{array}$ & P4 & $\begin{array}{l}\text { Mise en place/développement des services } \\
\text { de vulgarisation }\end{array}$ \\
\hline & Réglementations & P5 & $\begin{array}{l}\text { Restrictions à la mise hors production de } \\
\text { terres agricoles }\end{array}$ \\
\hline
\end{tabular}

Au sein du premier groupe de mesures, une distinction est établie entre celles qui agissent directement sur les prix des produits alimentaires, telles que les réformes des droits de douane, des taxes et des subventions ou l'application de mesures de contrôle des prix (M1-M4), et celles qui exercent une incidence sur les prix en agissant soit sur les quantités de produits alimentaires disponibles sur le marché, comme c'est le cas de la constitution de réserves de céréales, soit sur l'intensité de la demande du produit considéré (M5-M9). Cette distinction tient à ce que les premières ont un impact plus direct sur les prix alors que l'effet des secondes dépend de l'élasticité de l'offre et de la demande. D'après la théorie économique, dans une petite économie ouverte, les variations de l'offre et de la demande intérieures telles que celles entraînées par un déblocage des stocks de produits alimentaires n'ont guère de probabilité de modifier sensiblement les prix. Les interventions destinées à influer sur l'offre et la demande ne pourront 
probablement avoir d'effet que si les autres mesures prises par les pouvoirs publics limitent la transmission des cours internationaux des produits de base sur le marché intérieur. La classification distingue également les mesures prises aux frontières pour influer sur les échanges commerciaux, qu'il s'agisse des importations (M1 et M5) ou des exportations (M2 et M6), de celles axées sur le marché intérieur (M3, M4, M7, M8, M9).

La réduction des droits de douane compte parmi les mesures les plus aisées à mettre en œuvre d'un point de vue administratif. C'est probablement la mesure la plus largement adoptée, puisque 43 des 81 pays examinés dans l'enquête effectuée pour le compte de la FAO ont déclaré l'avoir mise en œuvre (Demeke et al., 2008). L'efficacité de cette mesure dépend du niveau initial des droits de douane et de l'ampleur de la réduction. Il est d'autant plus probable qu'une telle disposition ait un impact sur les prix que le niveau de départ des droits de douane sera élevé et que leur réduction sera forte. La réduction de la TVA est en règle générale plus difficile à mettre en œuvre que celle des droits de douane, tant d'un point de vue administratif que sous un angle pratique. Son efficacité dépend également du niveau initial de la TVA et de la réduction qui lui est apportée. Les mesures d'ordre fiscal ne contribuent efficacement à la baisse des prix alimentaires qu'à condition que le secteur de la vente au détail de produits alimentaires soit concurrentiel, car les détaillants pourraient sinon profiter de leur position de force sur le marché pour accroître leurs marges, et que les consommateurs achètent effectivement leurs produits dans les supermarchés et autres points de vente au détail du circuit formel.

Cette classification ne met par contre pas clairement en évidence une importante distinction : celle qui doit être faite entre les mesures d'intervention sur le marché qui ont un impact sur l'ensemble des prix du marché et celles qui n'agissent que sur les prix à la consommation. Cette distinction est importante du fait que les mesures du premier groupe exerceront probablement une pression à la baisse des prix à la production - réduisant donc les revenus des agriculteurs, ce qui a pour effet de brouiller les signaux du marché incitant les exploitants à accroître leurs volumes de production - alors que tel n'est pas le cas de celles de la seconde catégorie. L'analyse des diverses catégories d'interventions sur le marché porte à croire que toutes les mesures sauf celles de la catégorie M3 - et notamment les réductions de TVA et les subventions générales aux prix - devraient exercer une pression à la baisse des prix à la production.

La classification distingue les subventions alimentaires non ciblées fournies indifféremment à tous les consommateurs (M3) de celles qui sont destinées à des populations spécifiques au travers de filets de sécurité $(\mathrm{C} 1$ et $\mathrm{C} 2)$ - «programmes de transfert non contributifs ciblés d'une manière ou d'une autre sur les populations pauvres ou vulnérables »(Grosh et al., 2008). Les subventions alimentaires universelles accordées à tout un chacun permettent d'atténuer plus rapidement l'impact immédiat des hausses de prix, mais elles se révèlent coûteuses car elles ne ciblent pas efficacement ceux qui ont réellement besoin d'un soutien. Au nombre des filets de sécurité financiers figurent les transferts en espèces, dont ceux soumis au respect de certaines conditions, ainsi que les programmes de distribution de bons ou tickets d'alimentation. La catégorie baptisée «aide alimentaire» recouvre les programmes de transferts alimentaires, les programmes travail contre nourriture, les programmes de restauration scolaire, ainsi que les subventions alimentaires ciblées. L'efficacité des filets de sécurité dépend de manière générale du nombre de personnes vulnérables qui en bénéficient et des volumes d'aide fournis.

Dans le cas des mesures axées sur la production, l'étude s'inspire du système de classification utilisé par l'OCDE pour les besoins de l'estimation du soutien aux producteurs (OCDE, 2008). Cette classification distingue les mesures selon qu'elles impliquent des transferts au titre de la production, comme c'est le cas de l'instauration de prix minimums, ou au titre des intrants. Les transferts au titre des intrants sont divisés en trois catégories en fonction du type d'intrants. Les transferts au titre des intrants variables incluent les subventions aux engrais et aux semences. Les réductions des droits de douane sur les intrants agricoles destinées à stimuler la production sont classées dans la catégorie P2 plutôt que dans la catégorie M1 du fait qu'elles n'affectent pas directement le prix des produits de base. Les transferts au titre 
de la formation de capital fixe incluent les crédits bonifiés accordés aux exploitants. Les transferts au titre des services utilisés sur l'exploitation correspondent à la valeur des services de vulgarisation fournis aux exploitants à titre gratuit ou à un prix inférieur à leur coût. La dernière catégorie regroupe les mesures prises par les pouvoirs publics sous la forme de réglementations. Celles-ci n'impliquent aucun transfert de ressources (qu'il s'agisse d'argent, d'intrants, de connaissances, etc.) au profit des producteurs.

\subsection{Estimation de l'impact budgétaire des mesures prises}

Après avoir identifié les différents types de mesures prises par les pouvoirs publics, il convient d'évaluer quelle en a été l'importance relative. Un moyen d'y parvenir consiste à estimer les conséquences budgétaires directes de chacune des mesures. Les impacts indirects, tels que la réduction des recettes de TVA ou du produit de l'impôt sur le revenu résultant de la baisse des prix entrainée par l'instauration de contingents d'exportation, ne sont pas pris en considération. Il est vrai que toutes les mesures prises par les pouvoirs publics n'ont pas de conséquences budgétaires, mais cette méthode n'en permet pas moins de comparer l'importance relative de celles qui ont de tels effets.

Les conséquences budgétaires tiennent aussi bien compte des augmentations des dépenses publiques, que ce soit au titre des subventions aux prix, des transferts en espèces ou du soutien aux producteurs, que des baisses des recettes publiques telles que celles entraînées par la réduction ou la suppression des droits de douane. Il peut par ailleurs arriver que certaines des mesures prises par les pouvoirs publics entraînent une augmentation des recettes budgétaires, comme c'est par exemple le cas de l'accroissement des taxes à l'exportation. Afin de bien distinguer ces deux types d'impacts budgétaires, il convient de souligner que les nombres positifs indiquent une augmentation des dépenses ou une réduction des recettes alors que ceux assortis d'un signe négatif correspondent à une diminution des dépenses ou à un accroissement des recettes. Les variations des positions budgétaires sont calculées pour 2007 et 2008.

Pour estimer la valeur marginale de la disposition considérée, les conséquences budgétaires sont essentiellement mesurées par la variation des dépenses et des recettes publiques. Lorsqu'une disposition des pouvoirs publics implique une augmentation des paiements au titre d'un programme existant, il est procédé à une estimation de la variation marginale des dépenses pour mesurer le coût de la mesure en question. Lorsqu'une mesure implique la réduction ou la suppression de certains droits de douane, la perte de recettes publiques est estimée en multipliant le nombre de points de variation des taux de droits par la valeur des importations relevant de la ligne tarifaire correspondante enregistrées pendant la période de mise en œuvre de la mesure en question. Il faut bien entendu prendre soin de ne pas tenir compte de la valeur des importations déjà soumises à des taux de droits plus bas en vertu d'accords préférentiels. En cas d'instauration de taxes à l'exportation ou d'augmentation de leur montant, l'accroissement des recettes est estimé en multipliant la valeur des exportations enregistrées durant la période de mise en œuvre de cette mesure par le nombre de points d'augmentation du taux d'imposition. ${ }^{1}$ Les effets de la réduction ou de la

1. Cette méthode devrait normalement surestimer l'accroissement des recettes entraîné par une augmentation d'une taxe à l'exportation du fait qu'elle ne tient pas en compte l'impact négatif sur le volume des exportations. Il a toutefois été jugé qu'elle offrait une approche satisfaisante pour les six pays pour lesquels les variations des recettes fiscales liées aux modifications apportées aux taxes à l'exportation sont ici calculées. Quatre de ces pays - Chine, Inde, Russie et Vietnam - ont instauré des taxes à l'exportation, et non pas relevé le taux de taxes préexistantes, de sorte qu'aucune recette budgétaire n'en était tirée auparavant. Autrement dit, les volumes exportés auraient peut-être été plus élevés sans la taxe, mais ils n'auraient pas généré de recettes budgétaires. Dans le cas de l'Indonésie, les taxes à l'exportation ont été relevées par rapport à un très bas niveau de départ (1.5\%), de sorte que les recettes qui auraient pu être tirées si les volumes exportés avaient été plus grands aurait probablement été minime eu égard au faible montant initial de la taxe. En Argentine, les taxes à l'exportation initiales étaient relativement élevées, 20\% ou plus pour les produits considérés, et elles constituent une importante source de recettes publiques. Cependant, les contingents et l'obligation d'enregistrement restreignent également les exportations. C'est au travers de ces instruments que les pouvoirs publics régulent le volume des exportations. Les négociants 
suspension des restitutions à l'exportation, qui entrainent une diminution des dépenses budgétaires, sont calculés de manière similaire. En l'occurrence, la valeur des exportations est multipliée par le nombre de points de diminution du taux de restitution. Les statistiques budgétaires ne révèlent donc pas l'intégralité des coûts d'un programme ou des recettes générées par des droits de douane à l'importation ou par une taxe à l'exportation.

Un certain nombre de pays, tels que le Brésil, la Chine, l'Inde, la Russie et l'Ukraine, ont opté pour une augmentation des prix minimums de soutien ou d'intervention versés aux exploitants. Les produits achetés à ces prix par les organismes publics sont souvent revendus sur le marché libre afin d'atténuer les variations saisonnières ou régionales des cours. Les informations concernant le prix de revente du produit sur le marché et les coûts de stockage, de transport, etc., ne sont pas aisément disponibles. Les conséquences budgétaires d'une augmentation des prix minimums sont donc calculées au premier point de vente, la quantité achetée aux producteurs étant multipliée par l'augmentation marginale des prix, et elles sont classées dans la catégorie P1. Le montant net des recettes tirées de la vente du produit ne peut être déterminé, mais les autres coûts associés à la mise en œuvre du programme d'achats ne sont pas non plus pris en compte. L'Inde constitue une exception de taille : les achats de riz et de blé visent principalement à permettre leur distribution aux consommateurs pauvres à des prix bonifiés dans le cadre du système de distribution publique ciblée (Targeted Public Distribution System-TPDS). Les informations sur les quantités de produits redistribuées et sur la valeur du programme de subventions alimentaires sont aisément disponibles. En l'occurrence, l'augmentation des dépenses au titre des subventions alimentaires est classée dans la catégorie $\mathrm{C} 2$. Les achats de produits dépassant les quantités nécessaires au TPDS sont néanmoins calculés comme pour les autres pays et comptabilisés dans la catégorie P1.

\subsection{Quelles ont été les mesures prises par les pouvoirs publics?}

Le tableau 1.2 offre une synthèse des mesures à court terme prises dans chacun des dix pays pour répondre à la hausse des prix agricoles. Huit des dix pays ont pris des mesures pour agir directement sur le prix de produits agricoles de base ou pour en accroître l'offre, l'Afrique du Sud et du Chili faisant figure d'exception. Le Brésil, la Chine, l'Inde, l'Indonésie, la Russie et le Vietnam on tous réduit ou supprimé les droits de douane sur certains produits de base, et les dispositions dans ce sens sont pour certaines encore en place. Les mesures de réduction les plus larges, du point de vue du nombre de lignes tarifaires couvertes, ont été prises au Vietnam. Bien que ces dispositions ne soient pas considérées dans ce rapport comme des mesures prises pour répondre à la hausse des prix, l'Ukraine a procédé le 16 mai 2008, dans le cadre de ses engagements d'accession à l'OMC, à une réduction des droits de douane appliqués sur un vaste éventail de produits agricoles de base. L'Argentine, la Chine, l'Inde, l'Indonésie, la Russie et le Vietnam ont instauré des taxes à l'exportation ou en ont accru le montant, ou bien ils ont réduit les incitations par les prix fournies pour encourager les exportations. Les autorités monétaires des dix pays ont prix des mesures pour maîtriser l'inflation. Huit des dix pays ont mis en place des filets de sécurité destinés à protéger les consommateurs. À l'exception du Chili, tous les pays ont mis en œuvre des mesures de soutien de la production.

Dans les tableaux consacrés à chaque pays qui figurent à l'annexe $\mathrm{A}$, le coût budgétaire de chacune des mesures est, s'il y a lieu, indiqué en unités monétaires locales (UML). Le tableau 1.3 calcule le coût budgétaire total de l'ensemble des mesures prises par chacun des pays et le présente sous trois formats usuels afin de faciliter les comparaisons entre les pays. Son montant total est exprimé en une même monnaie (dollars des États-Unis) afin de donner une idée de l'ampleur des mesures prises. Il est également indiqué en pourcentage des recettes budgétaires pour en montrer le coût pour les pouvoirs publics, mais aussi par habitant pour en faire apparaître le coût pour l'ensemble de la population. Cette dernière valeur

n'auraient donc sans doute pas pu exporter de plus grands volumes que cela n'a été le cas, même si les droits de douane à l'exportation étaient demeurés inchangés. 
est mesurée en dollars internationaux et en PPA afin de permettre une comparaison des dépenses par habitant d'un pays à l'autre.

Dans tous les pays sauf l'Argentine, le coût budgétaire a sensiblement augmenté entre 2007 et 2008 au fur et à mesure que l'ampleur des hausses de prix s'intensifiait et que les pays ont eu recours à un plus large éventail de mesures. Pour l'Argentine, l'accroissement des recettes publiques généré par l'augmentation des taux de taxes à l'exportation est, d'après les estimations, légèrement supérieur au montant des dépenses additionnelles imposées par les interventions sur le marché et par les mesures de soutien à la production. Dans les neuf autres pays, la valeur des mesures prises par les pouvoirs publics variait, d'après les estimations, entre $0.1 \%$ des recettes budgétaires pour le Chili et $19 \%$ pour l'Inde, et se situait aux environs de $0.5 \%$ à $2.5 \%$ dans tous les autres cas. L'augmentation des dépenses au titre des programmes de subventions aux produits alimentaires et aux engrais a contribué pour $70 \%$ à l'alourdissement des coûts budgétaires liés aux mesures prises en Inde en 2008. En 2008, la valeur totale cumulée des dépenses budgétaires consacrées à ces deux programmes, et non uniquement l'augmentation marginale indiquée au tableau 1.3 et au tableau A.5 de l'annexe, représentait $22 \%$ des recettes fiscales et $2.2 \%$ du PIB. 
Tableau 1.2 Synthèse des mesures à court terme prises pour répondre à la hausse des prix agricoles, 2006-08

\begin{tabular}{|c|c|c|c|c|c|c|c|c|c|c|c|c|}
\hline $\begin{array}{l}\text { Grands types } \\
\text { de mesures }\end{array}$ & Catégories & Étiquette & Argentine & Brésil & Chili & Chine & Inde & Indonésie & Russie & $\begin{array}{l}\text { Afrique } \\
\text { du Sud }\end{array}$ & Ukraine & Vietnam \\
\hline \multirow{9}{*}{$\begin{array}{c}\text { Interventions } \\
\text { sur le marché } \\
\text { pour limiter la } \\
\text { hausse des } \\
\text { prix } \\
\text { alimentaires }\end{array}$} & $\begin{array}{l}\text { Agit directement sur le prix des produits } \\
\text { de base - importations }\end{array}$ & M1 & & $\checkmark$ & & $\checkmark$ & $\checkmark$ & $\checkmark$ & $\checkmark$ & & & $\checkmark$ \\
\hline & $\begin{array}{l}\text { Agit directement sur le prix des produits } \\
\text { de base - exportations }\end{array}$ & M2 & $\checkmark$ & & & $\checkmark$ & $\checkmark$ & $\checkmark$ & $\checkmark$ & & & $\checkmark$ \\
\hline & $\begin{array}{l}\text { Agit directement sur le prix des produits } \\
\text { de base - mesure de nature budgétaire }\end{array}$ & M3 & $\checkmark$ & $\checkmark$ & & & & $\checkmark$ & $\checkmark$ & & & \\
\hline & $\begin{array}{l}\text { Agit directement sur le prix des produits } \\
\text { de base - mesure non budgétaire }\end{array}$ & M4 & $\checkmark$ & & & $\checkmark$ & & & $\checkmark$ & & $\checkmark$ & \\
\hline & $\begin{array}{l}\text { Accroître/maintenir l'offre intérieure de } \\
\text { produits de base - importations }\end{array}$ & M5 & & & & & $\checkmark$ & $\checkmark$ & $\checkmark$ & & & \\
\hline & $\begin{array}{l}\text { Accroître/maintenir l'offre intérieure de } \\
\text { produits de base - exportations }\end{array}$ & M6 & $\checkmark$ & & & $\checkmark$ & $\checkmark$ & $\checkmark$ & & & $\checkmark$ & $\checkmark$ \\
\hline & $\begin{array}{l}\text { Accroître/maintenir l'offre intérieure de } \\
\text { produits de base - stocks }\end{array}$ & M7 & & $\checkmark$ & & $\checkmark$ & $\checkmark$ & $\checkmark$ & $\checkmark$ & & $\checkmark$ & \\
\hline & $\begin{array}{l}\text { Diminuer la demande non alimentaire du } \\
\text { produit de base considéré }\end{array}$ & M8 & & & & $\checkmark$ & $\checkmark$ & & & $\checkmark$ & & \\
\hline & Améliorer le fonctionnement du marché & M9 & & & $\checkmark$ & $\checkmark$ & $\checkmark$ & $\checkmark$ & $\checkmark$ & & $\checkmark$ & $\checkmark$ \\
\hline $\begin{array}{l}\text { Maîtriser } \\
\text { l'inflation }\end{array}$ & Impact sur l'ensemble des prix & I1 & $\checkmark$ & $\checkmark$ & $\checkmark$ & $\checkmark$ & $\checkmark$ & $\checkmark$ & $\checkmark$ & $\checkmark$ & $\checkmark$ & $\checkmark$ \\
\hline \multirow{2}{*}{$\begin{array}{c}\text { Filets de } \\
\text { sécurité pour } \\
\text { les } \\
\text { consomma- } \\
\text { teurs } \\
\end{array}$} & Aide pécuniaire & $\mathrm{C} 1$ & & $\checkmark$ & $\checkmark$ & $\checkmark$ & & $\checkmark$ & $\checkmark$ & $\checkmark$ & $\checkmark$ & \\
\hline & Aide alimentaire & $\mathrm{C} 2$ & & & & & $\checkmark$ & $\checkmark$ & $\checkmark$ & $\checkmark$ & $\checkmark$ & \\
\hline \multirow{5}{*}{$\begin{array}{l}\text { Mesures axées } \\
\text { sur la } \\
\text { production }\end{array}$} & $\begin{array}{l}\text { Transferts au titre de la production de } \\
\text { produits de base }\end{array}$ & P1 & $\checkmark$ & $\checkmark$ & & $\checkmark$ & $\checkmark$ & $\checkmark$ & $\checkmark$ & & $\checkmark$ & \\
\hline & Transferts au titre des intrants variables & $\mathrm{P} 2$ & & $\checkmark$ & & $\checkmark$ & $\checkmark$ & $\checkmark$ & & $\checkmark$ & & $\checkmark$ \\
\hline & $\begin{array}{l}\text { Transferts au titre de la formation de } \\
\text { capital fixe }\end{array}$ & P3 & & $\checkmark$ & & $\checkmark$ & $\checkmark$ & & & $\checkmark$ & & \\
\hline & $\begin{array}{l}\text { Transferts au titre des services utilisés sur } \\
\text { l'exploitation }\end{array}$ & $\mathrm{P} 4$ & & $\checkmark$ & & & $\checkmark$ & & & $\checkmark$ & & \\
\hline & Réglementations & P5 & & & & $\checkmark$ & & & & & & $\checkmark$ \\
\hline
\end{tabular}


Tableau 1.3. Conséquences budgétaires des mesures prises pour répondre à la hausse des prix alimentaires, 2007 et 2008

\begin{tabular}{|c|c|c|c|c|c|c|c|c|c|c|}
\hline Année & Argentine & Brésil & Chili & Chine & Inde & Indonésie & Russie & $\begin{array}{c}\text { Afrique } \\
\text { du Sud }\end{array}$ & Ukraine & Vietnam \\
\hline \multicolumn{11}{|c|}{ Coût budgétaire (millions USD) } \\
\hline 2007 & 49 & 743 & 0 & 436 & 5273 & 644 & -32 & 786 & 79 & 48 \\
\hline 2008 & -122 & 2394 & 56 & 7813 & 24000 & 2095 & 2309 & 1849 & 246 & 242 \\
\hline \multicolumn{11}{|c|}{ Part des recettes budgétaires $(\%)$} \\
\hline 2007 & 0.1 & 0.2 & 0.0 & 0.1 & 3.8 & 0.8 & 0.0 & 0.9 & 0.2 & 0.3 \\
\hline 2008 & -0.1 & 0.6 & 0.1 & 1.7 & 19.1 & 2.1 & 0.6 & 2.4 & 0.6 & 1.0 \\
\hline \multicolumn{11}{|c|}{ Coût budgétaire par habitant (dollars internationaux, PPA) } \\
\hline 2007 & 3 & 5 & 0 & 1 & 12 & 6 & 0 & 27 & 4 & 2 \\
\hline 2008 & -5 & 16 & 5 & 11 & 55 & 16 & 22 & 67 & 10 & 7 \\
\hline
\end{tabular}

Source : Élaboré à partir des tableaux détaillés de l'annexe A et des données du FMI, Statistiques financières internationales (2010).

Graphique 1.1. Composition des mesures budgétaires prises pour répondre à la hausse des prix alimentaires, 2007 et 2008

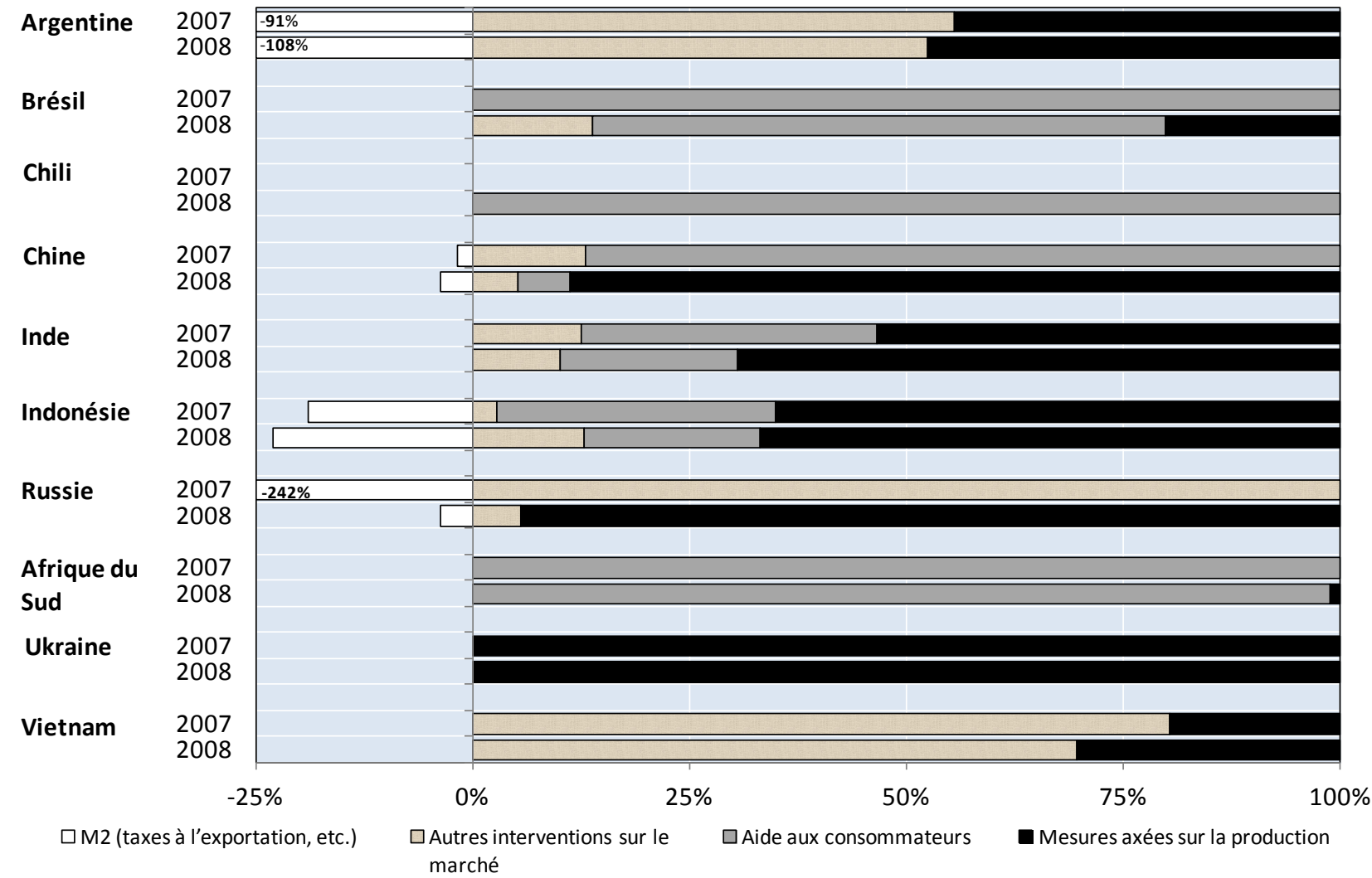

Notes : L'accroissement des recettes publiques généré par l'augmentation des taxes à l'exportation et la diminution des dépenses entraînée par la réduction des restitutions à l'exportation, c'est-à-dire par les mesures classées dans la catégorie M2 et assorties d'un signe négatif, sont exprimées en pourcentage des dépenses totales imposées par les autres catégories, afin d'indiquer jusqu'à quel point elles contrebalancent les augmentations des dépenses.

Les recettes générées par l'application de taxes à l'exportation sur le riz basmati en Inde et sur le riz au Vietnam ne sont pas prises en considération.

Source : Élaboré à partir des tableaux détaillés de l'annexe A consacrés aux divers pays. 
Le graphique 1.1 montre la composition des mesures budgétaires, réparties en quatre catégories : celles de la catégorie M2, les autres interventions sur le marché, les filets de sécurité pour les consommateurs, et les mesures axées sur la production. Les mesures de la catégorie M2 se distinguent des autres interventions sur le marché par le fait qu'elles génèrent un gain budgétaire (représenté ici par un signe négatif) - qu'il résulte d'une hausse des recettes publiques grâce, par exemple, à l'instauration de taxes à l'exportation ou à l'augmentation de leur montant, ou d'une diminution des dépenses grâce, par exemple, à la réduction ou à la suspension des restitutions à l'exportation. Les gains budgétaires tirés de ces mesures sont indiqués sous la forme de leur part en pourcentage par rapport aux dépenses budgétaires imposées par toutes les autres catégories de mesures, afin de montrer jusqu'à quel point ils contrebalancent les augmentations des dépenses.

Les interventions sur le marché, autres que celles répertoriées dans la catégorie M2, occupent une place relativement importante en Argentine, en Russie et au Vietnam. En Russie et au Vietnam, c'est principalement là une conséquence de la diminution des recettes budgétaires imputable aux réductions des droits de douane, alors qu'en Argentine c'est le résultat d'une augmentation des dépenses consacrées aux subventions aux transformateurs. Pour l'Afrique du Sud, le Brésil, et le Chili, comme pour la Chine en 2007, les filets de sécurité pour les consommateurs ont pesé lourd d'un point de vue budgétaire, mais elles ont également représenté plus d'un quart des dépenses en Inde et en Indonésie. Les mesures de soutien aux producteurs ont une place prépondérante dans les dépenses budgétaires en Chine, en Inde, en Indonésie, en Russie et en Ukraine, mais aussi dans une moindre mesure en Argentine et au Vietnam. Ces sept pays ont renforcé les mesures de contrôle des exportations.

L'Argentine a privilégié les mesures de restriction des exportations, dont l'instauration de taxes à l'exportation, de contingents et de systèmes de licences (tableau A.1 de l'annexe). Déjà en vigueur depuis quelque temps, ces mesures ont été ajustées pour répondre à la hausse des cours mondiaux. Le graphique 1.2 montre les modifications apportées aux taxes à l'exportation sur le blé, le maïs, et le soja et ses dérivés. Les taxes à l'exportation sur le soja et ses dérivés ont été successivement augmentées pour accroître les recettes publiques et pour dissuader les exploitants de se détourner de la production de céréales au profit de celle de soja. Conformément à la politique antérieurement appliquée, les recettes budgétaires générées par l'augmentation des taxes à l'exportation ont servi à fournir un soutien aux transformateurs (afin de les dédommager du maintien d'un bas niveau de prix dans le cadre des «accords de prix ») et aux producteurs (pour le maintien sur le marché intérieur de prix plus bas qu'ils ne devraient l'être). 
Graphique 1.2. Taxes à l'exportation sur les céréales et le soja en Argentine, 2006-09

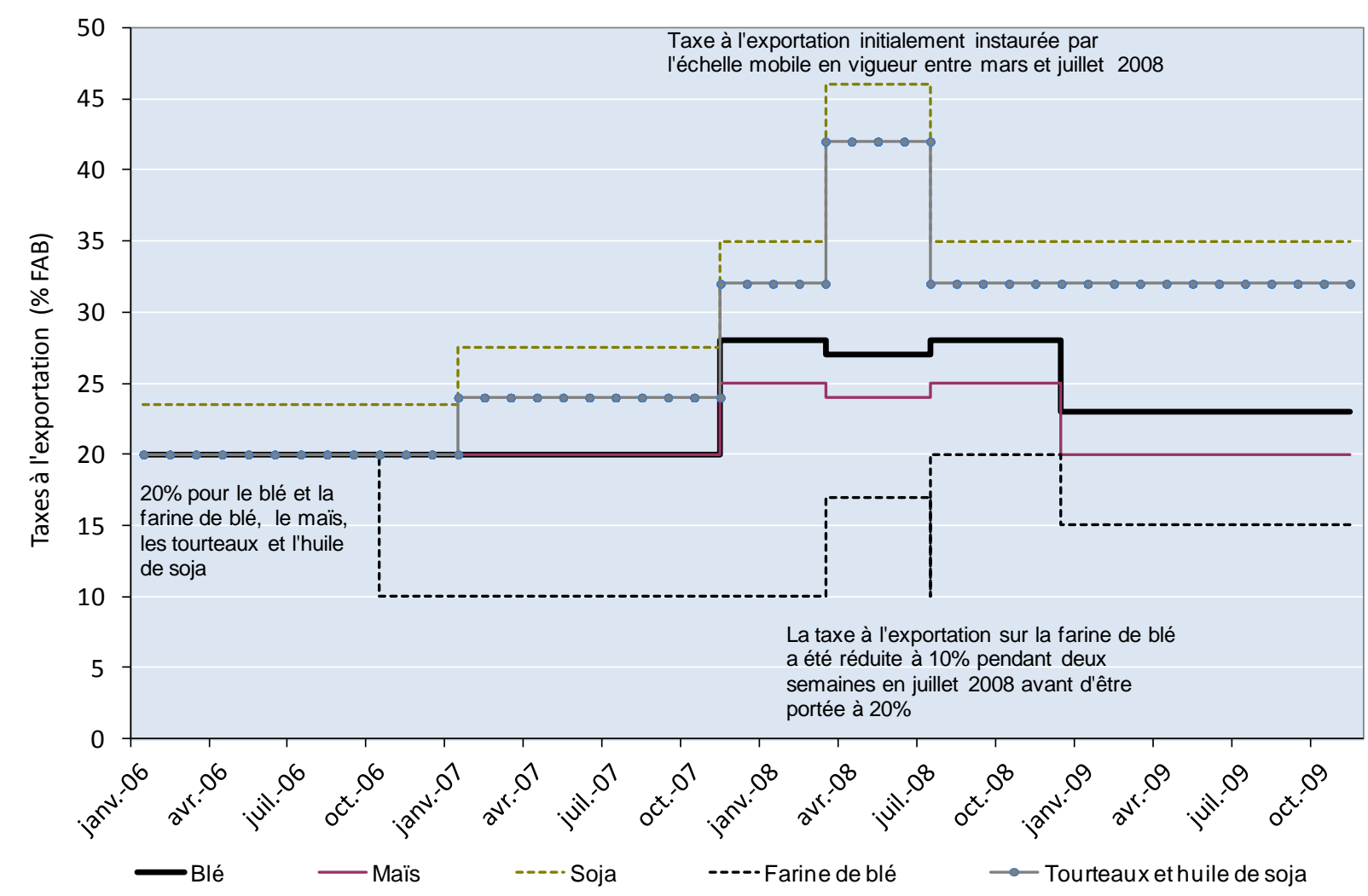

Source : Élaboré par l'auteur à partir des informations issues des rapports du Réseau d'information sur l'agriculture mondiale (Global Agriculture Information Network - GAIN) du Service de l'agriculture extérieure de I'USDA.

Le Brésil a quant à lui principalement accru les paiements au titre du programme Bolsa Família (tableau A.2 de l'annexe et graphique 1.3). Créé en octobre 2003, Bolsa Família est un programme de transferts conditionnels en espèces au profit de plus de 12 millions de familles (50 millions de personnes), soit un quart de la population. Le montant de ces paiements a été maintenu constant depuis 2003 jusqu'en juillet 2007, malgré une augmentation de $16.7 \%$ du coût de la vie. L'augmentation des dépenses enregistrée durant la période 2004-06 est imputable au nombre croissant de bénéficiaires. En juillet 2007, le décret 6.157 a accru de $17 \%$ à $20 \%$ (selon les catégories) le montant des paiements, qui ont ainsi retrouvé leur niveau initial en termes réels (Grosh et al., 2009). Le montant des paiements a de nouveau été augmenté en 2008 et 2009. Près de $90 \%$ en sont utilisés pour l'achat de nourriture. D'autres mesures importantes ont consisté à instaurer un contingent tarifaire exempt de droits pour le blé, à réduire les taxes et redevances sur le blé et la farine de blé, à ouvrir de nouvelles lignes de crédit dans le cadre du programme «Plus de nourriture», et à augmenter les prix minimums garantis pour un large éventail de produits de base pour la campagne 2008/09. Le gouvernement considère que le Brésil est l'un des rares pays capables d'accroître la production à grande échelle pour répondre à la demande mondiale croissante de produits alimentaires. 
Graphique 1.3. Dépenses et bénéficiaires du programme Bolsa Família, 2004-09

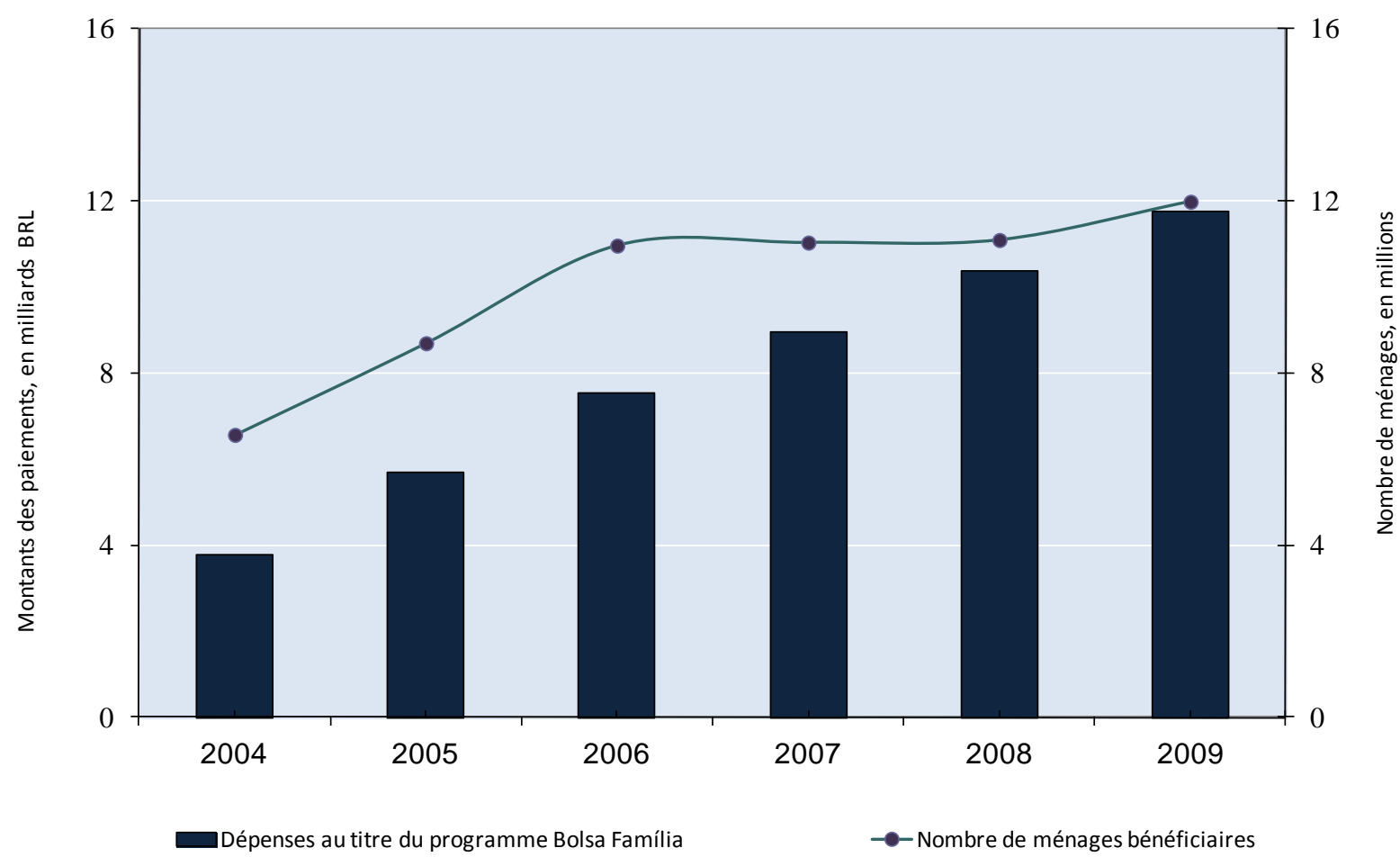

Source: Calculs de l'auteur sur la base du site web du programme Bolsa Família, Gouvernement du Brésil, www.mds.gov.br/bolsafamilial.

Les mesures mises en œuvre au Chili visaient principalement à accroître la transparence des prix, à fournir un certain soutien des revenus à ceux qui sont le plus durement touchés et à maîtriser l'augmentation globale de l'inflation (tableau A.3 de l'annexe). Les banques centrales de l'Afrique du Sud et du Chili ont été parmi les premières à augmenter les taux d'intérêts de la politique monétaire et parmi celles qui les ont le plus accrus (graphique 1.4). 
Graphique 1.4. Taux d'intérêts de la politique monétaire dans divers pays, 2006-09

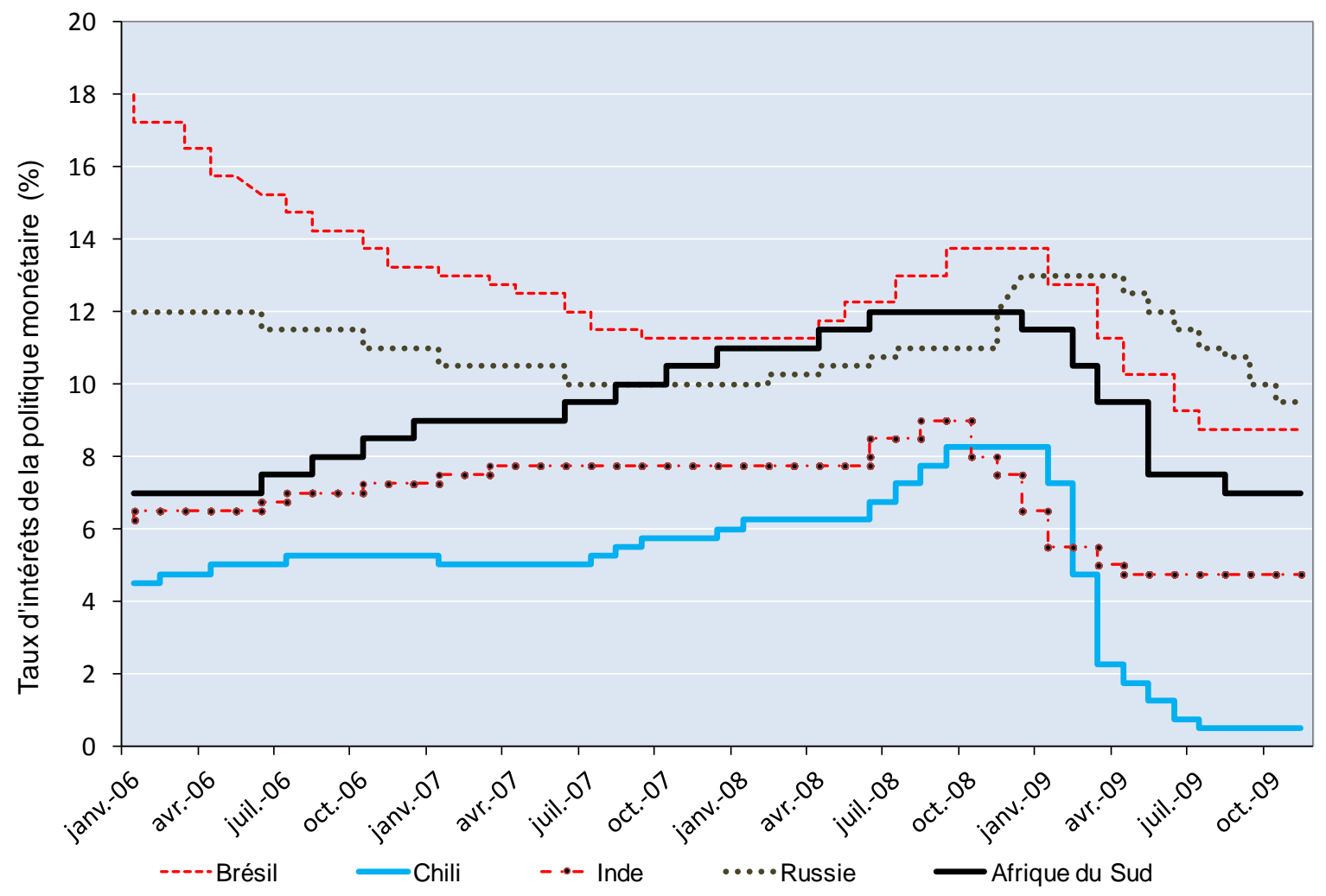

Source : Élaboré à partir des rapports annuels et des sites web officiels des autorités des banques centrales respectives.

Les premières mesures prises par la Chine étaient pour l'essentiel d'ordre intérieur : déblocage des stocks publics, soutien accru aux consommateurs au moyen de programmes ciblés, et moratoire sur la construction d'installations industrielles de transformation des céréales destinées, par exemple, à la production d'éthanol (tableau A.4 de l'annexe). Cette dernière mesure a été soutenue par la suppression des restitutions à l'exportation pour l'éthanol en janvier 2007. Ces initiatives ont rapidement été suivies par l'adoption d'une série de mesures aux frontières modifiant radicalement la position de la Chine en matière d'exportations de céréales puisqu'elles s'attachaient à les éviter au lieu de les encourager comme c'était le cas auparavant. Les restitutions à l'exportation de $13 \%$ dont bénéficiaient les céréales et le soja et ses dérivés ont été supprimées le 20 décembre 2007 et remplacées à compter du $1^{\mathrm{er}}$ janvier 2008 par des taxes de $5 \%$ à $25 \%$ sur les exportations de ces produits. Des restrictions quantitatives sous la forme de contingents d'exportation ont également été imposées, en particulier après août 2008, puis elles ont quasiment disparu depuis (Yang et al., 2008). Le soja, certaines huiles et quelques autres produits en nombre restreint ont bénéficié de réductions tarifaires, mais il n'en a pas été de même des céréales. Des mesures de contrôle des prix des céréales vivrières, des huiles végétales, de la viande de porc, de bœuf et de mouton, des produits laitiers et des œufs ont été imposés entre la fin janvier 2008 et le début décembre 2008. 
Graphique 1.5. Subventions aux intrants et prix planchers pour le riz et le blé en Chine 2005-09

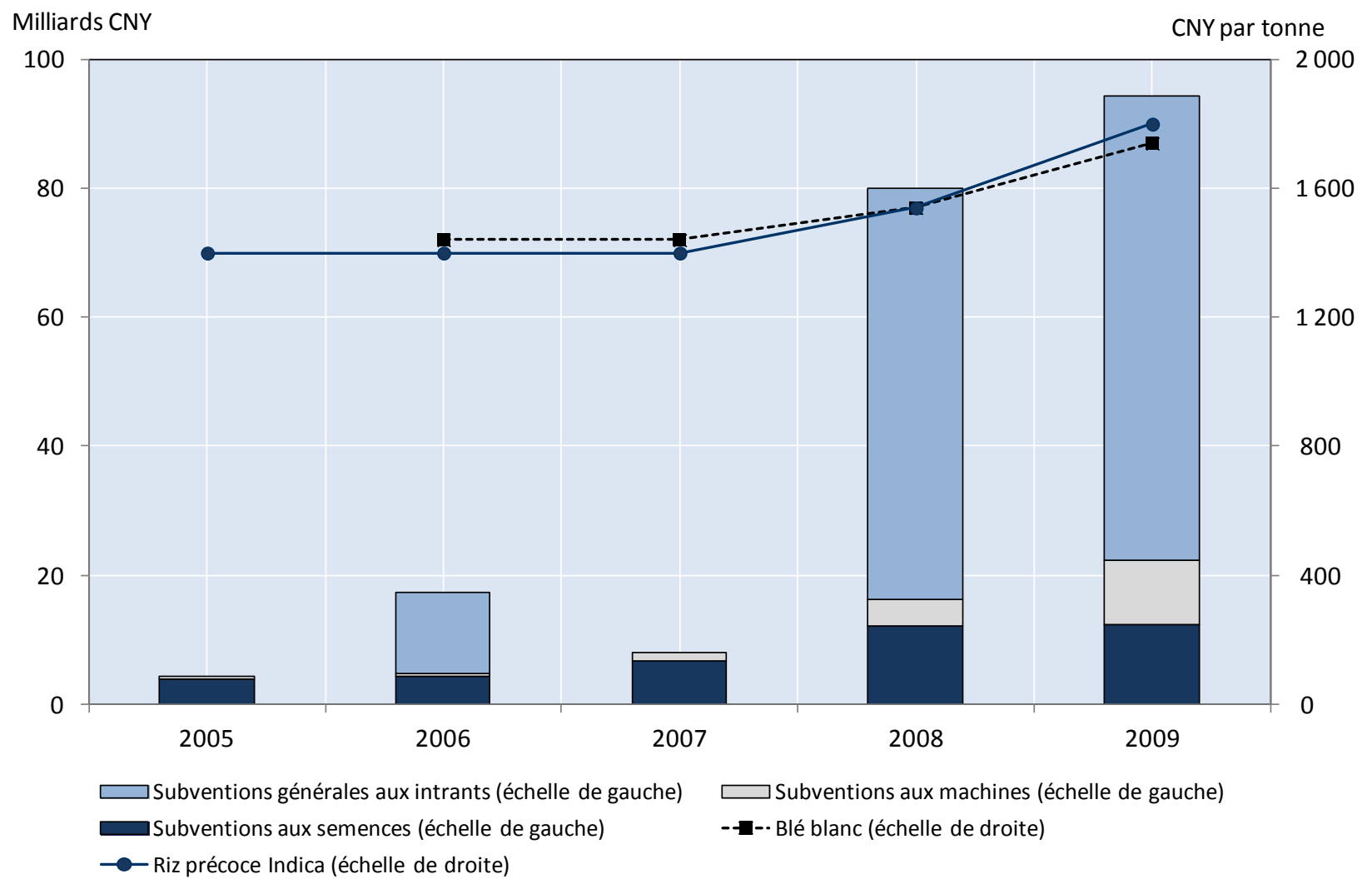

Source : Service de l'agriculture extérieure de I'USDA (USDA FAS), Grain and Feed Annual 2009, rapport CH9013.

Pour contrebalancer les mesures prises pour limiter les hausses de prix sur le marché intérieur, et afin de favoriser une augmentation de la production, le gouvernement a sensiblement accru le soutien à la culture de céréales (graphique 1.5). Les dépenses au titre des subventions générales aux intrants accordées aux exploitants sur la base des superficies consacrées à la production de céréales ont plus que doublé en 2008, passant de 28 à 64 milliards CNY. Les prix minimums du riz et du blé ont également été augmentés en 2008, pour la première fois depuis qu'ils ont été instaurés, en 2004 et 2006 respectivement. Les droits de douane sur les importations de produits d'alimentation animale ont été réduits afin d'aider les éleveurs.

Bon nombre des mesures prises en Inde visaient à sauvegarder son système de sécurité alimentaire très élaboré et en place de longue date (tableau A.5 de l'annexe). Un élément majeur en est le TPDS, qui distribue du blé et du riz subventionné aux pauvres (600 millions de personnes) au travers de son vaste réseau de «magasins à prix équitables ». Alors que le niveau des stocks régulateurs était inférieur aux «normes », les récoltes de blé relativement mauvaises de 2005 et 2006 ont conduit la Société de commerce d'État de l'Inde à cesser ses exportations de blé (auxquelles elle procédait les années précédentes afin de réduire ses vastes stocks excédentaires) pour importer 6.7 millions de tonnes afin de disposer d'approvisionnements suffisants pour les distributions publiques. Lorsque les cours mondiaux ont commencé à augmenter, le gouvernement a préféré ne pas en importer dans les mêmes quantités en 2007. Les exportations de blé et de produits du blé ont été interdites en février 2007, et celles de riz autre que le basmati début octobre 2007. ${ }^{2}$ Des prix minimums à l'exportation ont été instaurés pour le riz basmati début

2. L'interdiction des exportations de riz autre que le basmati a été remplacée à la fin octobre par un prix minimum à l'exportation de $425 \mathrm{USD} /$ tonne, montant d'environ $25 \%$ supérieur au niveau des cours mondiaux. Ce prix minimum à l'exportation a été relevé en décembre puis de nouveau en mars 2008, où il 
mars 2008, suivis par des restrictions concernant le port d'expédition à la mi-mars et par une taxe à l'exportation fin avril 2008 (qui a ensuite été supprimée le 20 janvier 2009). Les mesures de restriction des exportations de riz visaient à inciter les consommateurs à remplacer les aliments à base de blé par des aliments à base de riz. Des mesures d'interdiction des exportations de légumes secs, de poudres de lait et de maïs ont également été prises. Les droits à l'importation ont été sensiblement réduits pour le blé, le maïs, le riz et les huiles brutes ou raffinées de palme, de soja ou de tournesol. Cependant, compte tenu que le prix des céréales sur le marché intérieur était inférieur aux cours mondiaux, ces produits n'ont été importés qu'en très faibles quantités.

Pour encourager une augmentation de la production, les prix de soutien minimums applicables aux achats publics de riz et de blé ont été majorés (graphique 1.6), tout comme les dépenses au titre des subventions aux engrais. Augmenter les prix d'achat par les pouvoirs publics tout en maintenant à un niveau stable les prix de vente subventionnés appliqués aux personnes défavorisées dans le cadre du TPDS (ceux-ci sont restés constants depuis 2001) a eu pour conséquence de porter de 238 milliards INR en 2006/07 à 525 milliards INR en 2009/10 les dépenses générées par la subvention des produits alimentaires. De même, le maintien de la stabilité des prix des engrais pour les exploitants (ils n'ont pas augmenté depuis 2002) malgré la forte hausse des prix internationaux et des coûts intérieurs de production a entraîné ont abouti à une considérable augmentation des subventions aux engrais. Les dépenses au titre de ce programme sont passées de 224 milliards INR en 2006/07 à 758 milliards INR en 2008/09. Elles sont retombées à 500 milliards INR en 2009/10 à la suite de la baisse des prix internationaux des engrais. L'augmentation des volumes de production en 2007 et 2008 et les mesures de restriction des exportations ont permis aux pouvoirs publics de reconstituer leurs stocks de céréales pour les porter à des niveaux confortables et de créer une réserve stratégique de 5 millions de tonnes de céréales vivrières, en sus des normes en matière de stocks applicables dans le cadre du TPDS.

a atteint $650 \mathrm{USD} /$ tonne. Le $1^{\mathrm{er}}$ avril 2008, les pouvoirs publics sont revenus à une interdiction pure et simple des exportations de riz autre que le basmati. 


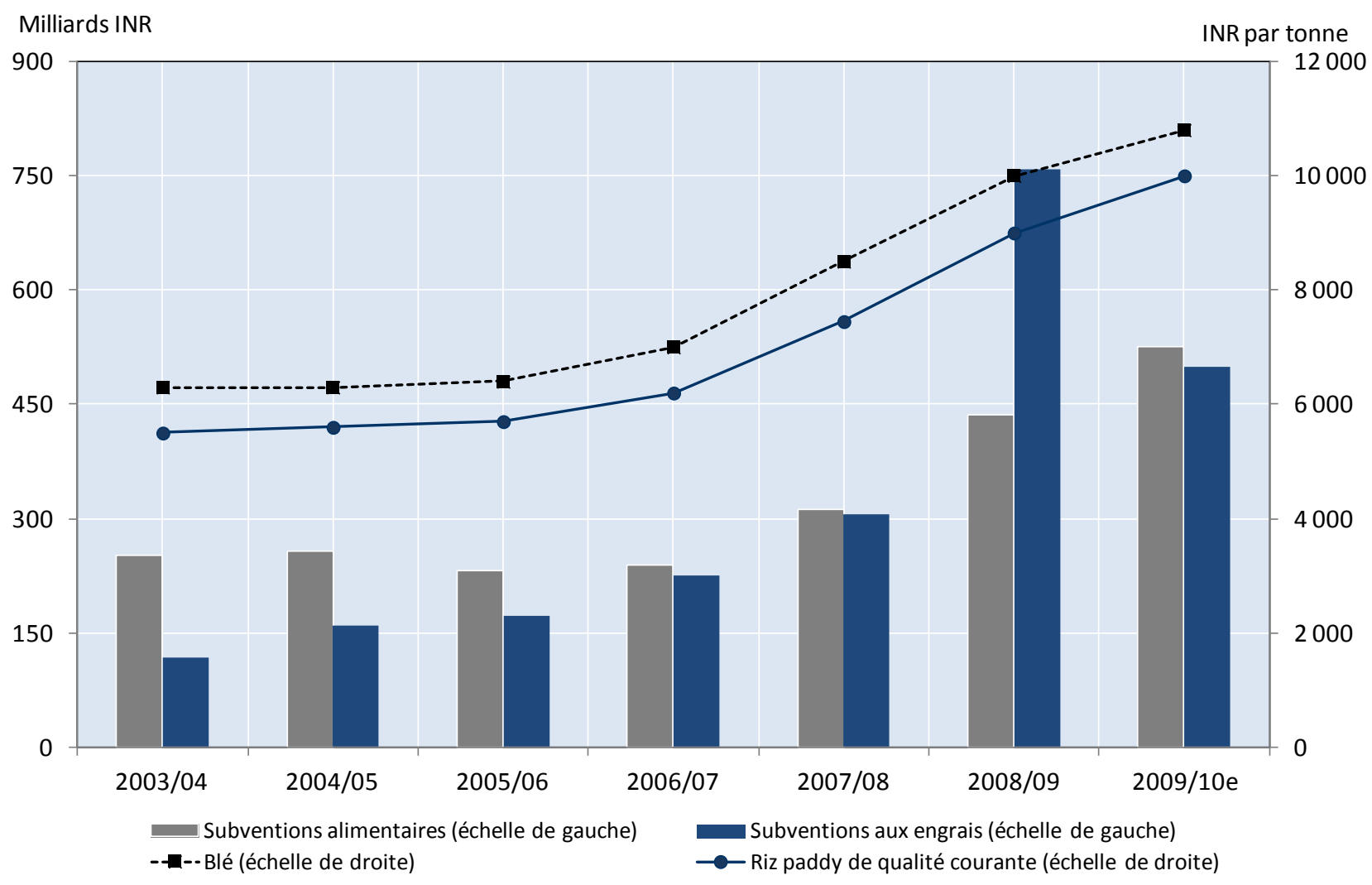

e : estimation.

Note : Les prix de soutien minimums incluent les primes incitatives annoncées durant les campagnes correspondantes. Source : Ministère des Finances, Economic Survey and Union Budget, diverses années.

En Indonésie, les pouvoirs publics ont eu recours à un vaste éventail de mesures qui ont affecté des produits de base très divers (tableau A.6 de l'annexe). Pour ce qui est du riz, les droits de douane ont été temporairement réduits, les stocks ont débloqués par l'organisme de commerce d'État (Bulog) qui dispose de pouvoirs discrétionnaires en matière d'importations, les prix d'achat de référence ont été augmentés et les prix des engrais ont été gelés. Une importante mesure ciblée a consisté à augmenter de $65 \%$ les quantités de riz subventionné distribuées dans le cadre du programme Rankin en 2008, par rapport à celles de 2007. Bien que l'Indonésie ait été par le passé le principal importateur au monde, place que les Philippines ne lui ont ravie que depuis peu, des mesures de contrôle des exportations de riz n'en ont pas moins été instaurées lorsque les cours mondiaux sont devenus plus élevés que les prix intérieurs. Diverses mesures ont été prises en ce qui concerne les produits oléagineux tropicaux. Les prix et les taxes de base à l'exportation applicables à l'huile de palme brute ont été relevés, de l'huile de friture subventionné a été mise sur le marché et distribuée à 19.1 millions de ménages pauvres, et la TVA a en outre été supprimée.

En Russie, les pouvoirs publics ont prix diverses mesures à la frontière (tableau A.7 de l'annexe). Les droits de douane ont été réduits pour les huiles de soja, de colza et de tournesol et pour le lait et les produits laitiers, et ils ont été supprimés pour les huiles tropicales. Des taxes à l'exportation ont été instaurées pour le blé et l'orge depuis la mi-novembre 2007 jusqu'au 30 juin 2008. Entre le 24 octobre 2007 et le 30 avril 2008, les prix des denrées alimentaires de première nécessité (dont le pain de blé, le pain de seigle, le lait, le kéfir, l'huile de tournesol en bouteille et les œufs de volaille) ont été «gelés » à leur niveau du 15 octobre dans le cadre d'un accord entre les pouvoirs publics et les principaux transformateurs et 
détaillants. En contrepartie, les pouvoirs publics ont appliqué aux transformateurs des taux d'intérêt bonifiés sur les avances de fonds de roulement pour l'achat de matières premières. Pour reconstituer les stocks d'intervention publics, qui avaient été débloqués et mis sur le marché en 2007/08, les prix d'achat des céréales par les autorités ont été relevés de $60 \%$ pour la campagne 2008/09. Des subventions ont été accordées en 2008 aux producteurs de porcs et de volailles pour compenser l'augmentation des coûts de l'alimentation des animaux.

Les mesures prises en Afrique du Sud ont largement privilégié l'augmentation des aides sociales (tableau A.8 de l'annexe et graphique 1.7). Ces aides font partie intégrante d'un filet de sécurité destiné à protéger les personnes aux stades vulnérables de la vie, tels que la vieillesse ou l'enfance, ou lorsqu'elles sont handicapées et qu'elles ne peuvent travailler. En 2007 et 2008, leur montant mensuel maximal a été augmenté de 5\%-6\% et 5\%-8\% respectivement. Ces dispositifs ont en outre été ajustés pour répondre au ralentissement de l'économie mondiale. Les seuils de revenu ont été majorés pour permettre aux personnes ayant des ressources légèrement plus élevées de bénéficier d'une aide. Par exemple, en août 2008, le seuil de revenus pour bénéficier de l'aide au titre des enfants à charge, qui n'avait pas été modifié depuis l'instauration de celle-ci en 1988, a été relevé - il a en fait été multiplié par deux pour tenir compte de l'inflation. Au lieu d'établir un nouveau seuil statique, les autorités ont préféré adopter une formule qui en fixe le niveau à 10 fois le montant de l'aide. La limite d'âge des enfants ouvrant droit à cette aide a été portée de 14 à 15 ans à compter du $1^{\text {er }}$ janvier 2009, ce qui a permis à 220000 enfants supplémentaires d'en bénéficier. À l'autre extrémité, l'âge à partir duquel les hommes peuvent bénéficier d'une pension de vieillesse est en passe d'être ramené de 65 à 60 ans - c'est-à-dire au même âge que pour les femmes - sur la période 2008-10.

Graphique 1.7. Dépenses et bénéficiaires des aides sociales en Afrique du Sud, 2004-09

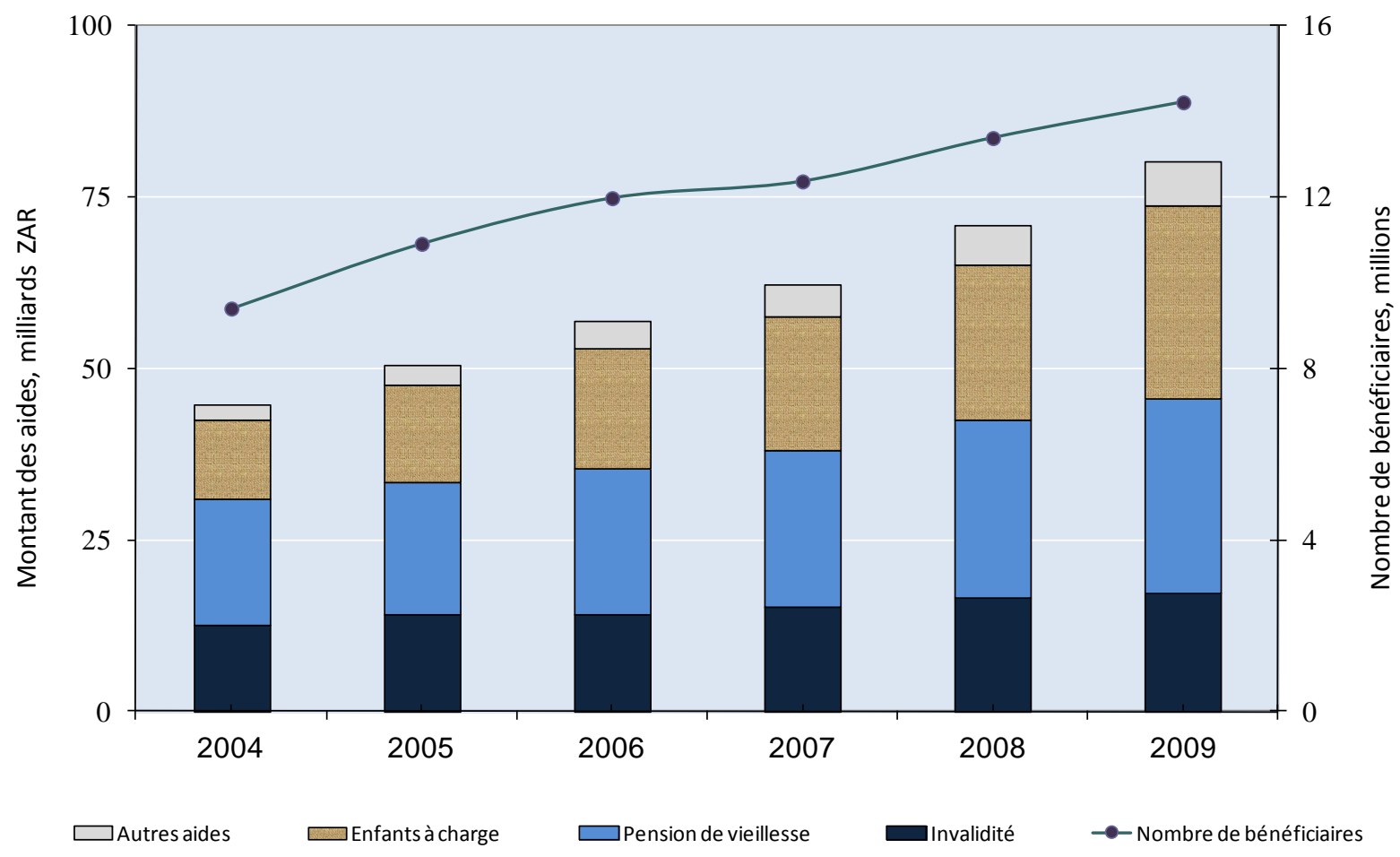

Source : Calculs de l'auteur d'après le Trésor national de l'Afrique du Sud (2010), Estimates of National Expenditure, Vote Social Development, diverses années, $\underline{w w w . i n f o . g o v . z a . ~}$ 
La principale mesure prise par le gouvernement ukrainien face à la hausse des prix alimentaires a consisté à imposer des contingents d'exportation des céréales (tableau A.9 de l'annexe et tableau 1.4). Les contingents d'exportation ont d'abord été instaurés à la fin septembre 2006, peu après la formation d'un nouveau gouvernement de coalition en août $2006 .{ }^{3}$ Après avoir été brièvement abolis au premier semestre 2007, les contingents d'exportation ont de nouveau été appliqués à compter du $1^{\mathrm{er}}$ juillet lorsqu'il est devenu évident que la récolte 2007 ne serait guère abondante en raison de la sécheresse et des températures ambiantes. Ils n'ont été supprimés que lorsqu'il est incontestablement apparu que la récolte 2008 serait exceptionnelle. Cette ligne de conduite allait néanmoins à l'encontre de l'engagement pris dans le cadre du protocole d'accession de l'Ukraine à l'OMC de lever toutes les mesures de restriction des exportations de céréales dès la date d'entrée du pays dans ladite Organisation (16 mai 2008). Le gouvernement a justifié les mesures de restriction des exportations par la nécessité de parvenir à : assurer la sécurité alimentaire ; garantir que les prix du pain demeurent peu élevés - le gouvernement était déterminé à tout faire pour en éviter l'augmentation avant les élections au parlement qui devaient avoir lieu sous peu, car certains y voient un indicateur de performance de l'action des pouvoirs publics; faire en sorte que les transformateurs de viande bénéficient d'orge et de maïs moins cher; et reconstituer les réserves de céréales de l'État.

3. Leur soudaine instauration a pris de nombreux producteurs et négociants par surprise. Un projet de résolution n'avait pas été publié par le ministère de l'Économie dans les délais exigés par la législation ukrainienne sur la politique réglementaire. Cette mesure a de fait compromis l'exécution des contrats d'exportation qui avaient déjà été signés. Les négociants de céréales ont donc été dans l'impossibilité de se conformer à leurs obligations contractuelles et ils ont subi de lourdes pertes financières, sans compter qu'ils n'ont pu écouler leurs stocks (UkrAgroConsult, 2009). 
Tableau 1.4. Mesures de restriction des exportations de céréales en Ukraine, 2006-08

\begin{tabular}{|c|c|c|c|c|c|c|}
\hline \multirow[b]{2}{*}{$\mathrm{AC}$} & \multirow[b]{2}{*}{ Mois } & \multicolumn{4}{|c|}{ Tonnes } & \multirow[b]{2}{*}{$\mathrm{CC}$} \\
\hline & & Blé & Maïs & Orge & Seigle & \\
\hline \multirow{4}{*}{ 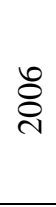 } & Sept. & \multicolumn{4}{|c|}{$\begin{array}{l}\text { Aucun contingent d'exportation n'était en place mais un système de licences d'exportation } \\
\text { et d'importation de blé et de blé-seigle (méteil) a été instauré le } 22 \text { septembre }\end{array}$} & \multirow{9}{*}{$\begin{array}{l}\text { o } \\
\varnothing \\
\vdots \\
0\end{array}$} \\
\hline & Oct. & & & & & \\
\hline & Nov. & $400000^{1}$ & $500000^{1}$ & $600000^{1}$ & $3000^{1}$ & \\
\hline & Déc. & & & & & \\
\hline \multirow{11}{*}{ ڤ્ণ } & Janv. & \multirow{4}{*}{$3000^{3}$} & \multirow{2}{*}{$500000^{2}$} & \multirow{2}{*}{$600000^{2}$} & \multirow{5}{*}{$3000^{4}$} & \\
\hline & Fév. & & & & & \\
\hline & Mars & & \multirow{3}{*}{ Aucun contingent } & \multirow{3}{*}{ Aucun contingent } & & \\
\hline & Avr. & & & & & \\
\hline & $\frac{\text { Ma1 }}{\text { Juin }}$ & Aucun contingent & & & & \\
\hline & Juil. & \multirow{6}{*}{$3000^{5}$} & \multirow{6}{*}{$3000^{5}$} & \multirow{6}{*}{$3000^{5}$} & \multirow{6}{*}{$3000^{5}$} & \multirow{12}{*}{ 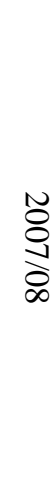 } \\
\hline & Août & & & & & \\
\hline & Sept. & & & & & \\
\hline & Oct. & & & & & \\
\hline & Nov. & & & & & \\
\hline & Déc. & & & & & \\
\hline \multirow{6}{*}{ 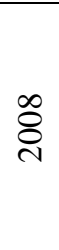 } & Janv. & \multirow{4}{*}{$200000^{8}$} & \multirow{3}{*}{$600000^{6}$} & \multirow{4}{*}{$400000^{8}$} & \multirow{6}{*}{$3000^{10}$} & \\
\hline & Fév. & & & & & \\
\hline & Mars & & & & & \\
\hline & Avr. & & \multirow{2}{*}{ Aucun contingent ${ }^{7}$} & & & \\
\hline & Mai & $1000000^{9}$ & & $500000^{9}$ & & \\
\hline & Juin & \multicolumn{3}{|c|}{ Suppression des contingents d'exportation } & & \\
\hline
\end{tabular}

1. Contingents du 17 octobre 2006 au 31 décembre 2006 - annoncés le 11 octobre.

2. Contingents du $1^{\text {er }}$ janvier 2007 au 30 juin 2007 - annoncés le 8 décembre - mais ils ont été supprimés le 22 février 2007.

3. Contingents du $1^{\text {er }}$ janvier 2007 au 30 juin 2007 - annoncés le 8 décembre - mais ils ont été supprimés le 16 mai 2007.

4. Contingent du $1^{\text {er }}$ janvier 2007 au 30 juin 2007 - annoncé le 8 décembre.

5. Contingents du $1^{\text {er }}$ juillet 2007 au 30 septembre 2007 - annoncés le 20 juin - mais ils ont été prolongés à deux reprises, d'abord jusqu'au $1^{\text {er }}$ novembre 2007 - mesure annoncée le 26 septembre - puis jusqu'au 31 décembre 2007 - mesure annoncée le 31 octobre.

6. Contingent du $1^{\mathrm{er}}$ janvier 2008 jusqu'au 31 mars 2008 - annoncé le 26 septembre.

7. Bien que les contingents d'exportation aient été éliminés pour le maïs, les exportations devaient avoir lieu dans le cadre d'un système de licences automatiques du $1^{\mathrm{er}}$ avril jusqu'au 30 juin 2008 mais cette exigence a été levée le 23 mai 2008 lorsque les contingents d'exportation ont été supprimés.

8. Contingents du $1^{\text {er }}$ janvier 2008 jusqu'au 31 mars 2008 - annoncés le 26 septembre - puis ils ont été prolongés jusqu'au 30 avril 2008 - mesure annoncée le 28 mars.

9. Une augmentation des quantités exportables dans le cadre des contingents a été autorisée jusqu'au 30 juin 2008 - mesure annoncée le 23 avril - mais les contingents d'exportation ont été supprimés le 23 mai 2008 - mesure annoncée le 21 mai.

10. Contingent du $1^{\mathrm{er}}$ janvier 2008 jusqu'au 31 mars 2008 - annoncé le 26 septembre - prolongé à deux reprises, d'abord jusqu'au 30 avril 2008 - mesure annoncée le 28 mars - puis jusqu'au 30 juin 2008 - mesure annoncée le 23 avril - avant d'être finalement supprimé le 23 mai 2008 - mesure annoncée le 21 mai.

Source : D'après Competitive Agriculture or State Control - Ukraine's Response to the Global Food Crisis, Note d'orientation, rapport $n^{\circ}$ 44984-UA, Banque mondiale, www.worldbank.org.ua, complété par des informations additionnelles tirées du Rapport UP8012 du Réseau d'information sur l'agriculture mondiale de l'USDA du 19 juin 2008.

$\mathrm{Au}$ Vietnam, les pouvoirs publics ont centré leur action sur le marché du riz, s'appuyant sur des mesures préexistantes pour maîtriser tant le volume que la valeur des exportations (tableau A.10 de l'annexe). L'organisme vietnamien en charge de l'alimentation (Vietnam Food Administration - VFA parfois appelée «Vietfood») administre le régime applicable aux exportations de riz, conformément aux instructions du Comité d'administration des exportations de riz. Le vice-ministre du Commerce préside ce Comité, qui réunit des représentants de la VFA, des services du premier ministre, du ministère des Finances, de celui de l'Agriculture et du Développement rural, de celui de la Planification et de l'Investissement, ainsi que de la Banque nationale du Vietnam. Des objectifs d'exportation sont fixés tous les ans et sont révisés en cours d'année en fonction du déroulement des récoltes. Ces objectifs tiennent de 
fait lieu de contingents d'exportation. Les négociants privés doivent enregistrer les contrats d'exportation auprès de la VFA en vue d'en obtenir l'agrément. Des prix minimums à l'exportation sont également fixés, bien que la VFA autorise parfois des contrats à des prix inférieurs. À la suite de la hausse des cours mondiaux, les objectifs d'exportation ont été réduits, l'enregistrement de nouveaux contrats a été régulièrement clos, les conditions d'agrément ont été durcies et les prix minimums à l'exportation ont été relevés. Le gouvernement a également établi un système de taxes à l'exportation pour le riz, qui a été mis en œuvre entre le 21 juillet 2008 et le 19 décembre 2009. Ces taxes n'étaient toutefois perçues que si le prix à l'exportation dépassait un certain seuil dont le niveau était inférieur à celui des prix à l'exportation, orientés à la baisse. À un niveau plus large, le Vietnam a réduit les droits de douane à l'importation applicables à un grand nombre de produits, dont la volaille, les poudres de lait, le maïs et l'huile de palme.

\subsection{Pourquoi les gouvernements ne prennent-ils pas tous des mesures identiques?}

Les raisons pour qu'un gouvernement réagisse et prenne des mesures pour faire face à la hausse des cours internationaux des produits alimentaires dépendent pour une large part des effets de celle-ci sur le pays, au plan national comme au niveau des ménages. Ces effets sont déterminés par divers facteurs dont le revenu national, l'incidence de la pauvreté, la part des dépenses consacrées à l'alimentation, la place relative du produit de base considéré dans la consommation globale, et la contribution de l'agriculture au PIB et à l'emploi (Benson et al., 2008). Les dix pays examinés présentent de considérables disparités sous chacun de ces aspects. Ils sont toutefois également dissemblables à d'autres points de vue, tels que les systèmes politiques en place, les capacités institutionnelles existantes, les expériences passées, l'échelle de valeurs dominante ou de la conception générale de l'action des pouvoirs publics. Bien que ces facteurs aient, parmi d'autres, eu un impact sur les mesures prises par les pouvoirs publics, ils ne sont guère quantifiables et ne sont donc pas pris en considération dans l'analyse ci-dessous.

Les quatre pays d'Asie se caractérisent par le plus bas revenu national par habitant et de manière générale par la plus forte incidence de la pauvreté (graphique 1.8). Le revenu national par habitant de l'Afrique du Sud est très similaire à celui du Brésil. Le pourcentage de la population vivant avec moins de 1.25 USD par jour est néanmoins trois fois plus élevé dans le premier de ces pays que dans le second. Moins de 1\% de la population vit avec moins de 1.25 USD par jour au Chili, en Russie et en Ukraine, bien que le revenu national par habitant de l'Ukraine soit inférieur de plus de la moitié à celui des deux autres pays. 
Graphique 1.8. Revenu national et pauvreté, 2005

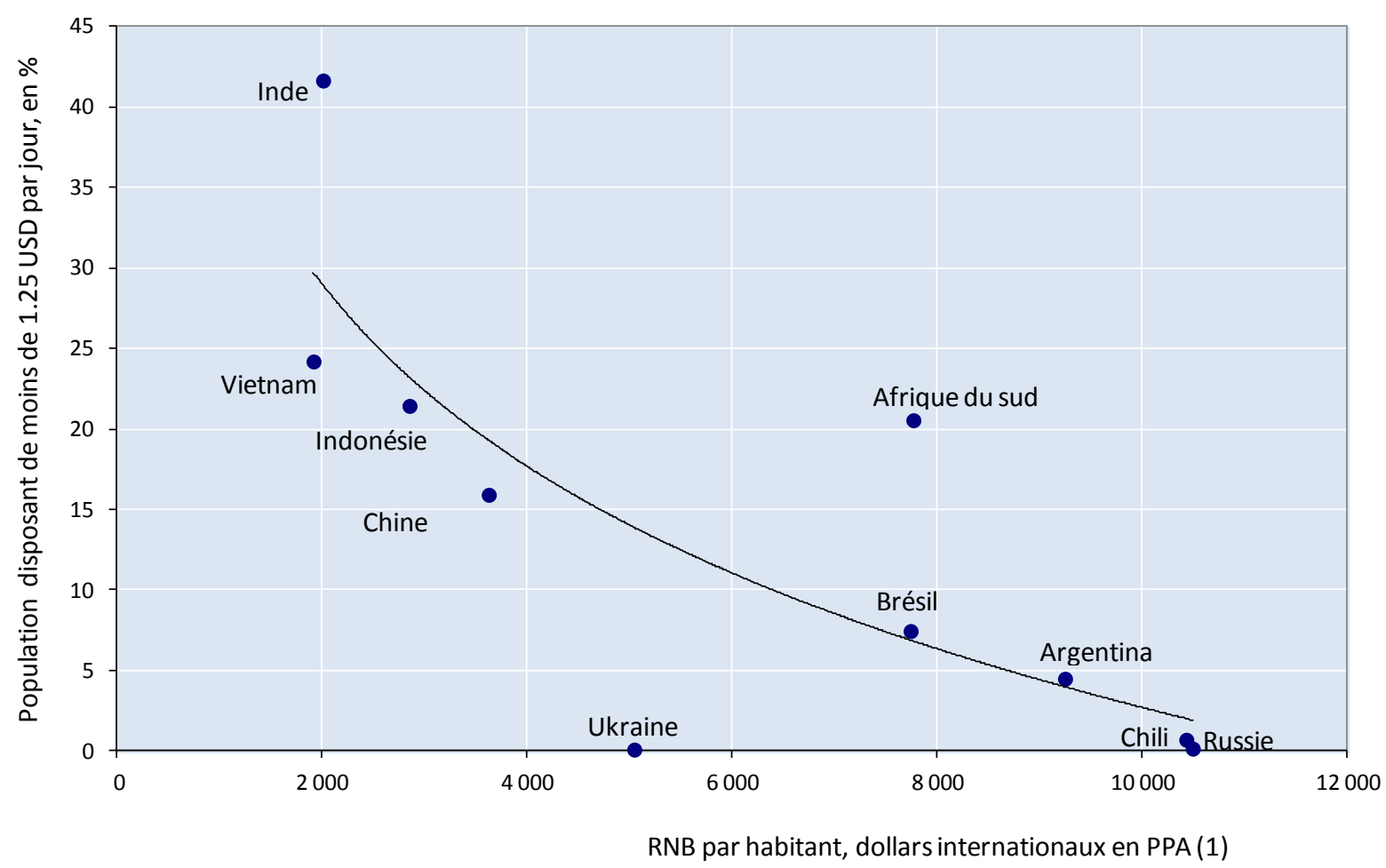

1. Un dollar international a le même pouvoir d'achat par rapport au RNB qu'un USD aux États-Unis. La Banque mondiale encourage l'utilisation de cette unité de compte pour mesurer avec précision le degré de pauvreté et de bien-être ; elle permet en effet de substituer les prix mondiaux à ceux observés au niveau local, rendant ainsi plus fidèlement compte de la valeur réelle du bien ou du service en question.

2. Le seuil de pauvreté international est converti en monnaie locale à l'aide des coefficients de conversion des PPA

Source : Banque mondiale, PovcalNet, 2010

L'alimentation compte généralement pour $40 \%$ à $50 \%$ des dépenses des ménages dans les quatre pays d'Asie (tableau 1.5). Bien que plus prospère si l'on en juge par le revenu par habitant, l'Ukraine est, des dix pays, celui où l'alimentation pèse le plus dans les dépenses des ménages. Dans les cinq autres pays, les ménages consacrent une plus faible part de leurs dépenses à l'alimentation. Il n'en demeure pas moins d'importantes disparités au sein de ce groupe. Bien que l'un et l'autre pays disposent d'un revenu national par habitant de niveau comparable, l'alimentation compte en Russie pour un tiers des dépenses des ménages contre moins d'un quart au Chili. De même, un ménage moyen consacre une bien plus grande part de son budget à l'alimentation en Argentine qu'en Afrique du Sud ou au Brésil, pourtant plus pauvres en termes de revenu par habitant.

Le blé constitue pour les ménages la principale source d'énergie alimentaire en Argentine, au Chili, en Russie et en Ukraine. L'Afrique du Sud est un cas à part parmi les dix pays, puisque le maïs y représente la principale source d'énergie alimentaire. Le riz est bien la principale source d'énergie dans les quatre pays d'Asie, mais il joue un rôle beaucoup plus essentiel en Indonésie et au Vietnam qu'en Chine ou en Inde, où les ménages consomment de plus grandes quantités de blé. Le Brésil se distingue des neuf autres pays en ceci qu'aucun produit alimentaire ne représente à lui seul plus de $13 \%$ de l'énergie apportée par l'alimentation, un large éventail de produits comptant pour des parts similaires. 
Tableau 1.5. Structure de la consommation alimentaire des ménages, 2003-05

\begin{tabular}{|c|c|c|c|c|c|c|c|c|c|}
\hline & \multirow{2}{*}{$\begin{array}{c}\text { Part des } \\
\text { dépenses des } \\
\text { ménages } \\
\text { consacrée à } \\
\text { l'alimentation } \\
(\%)\end{array}$} & \multicolumn{8}{|c|}{$\begin{array}{l}\text { Part de certains produits alimentaires dans la consommation d'énergie alimentaire }{ }^{1} \\
(\%)\end{array}$} \\
\hline & & Blé & Maïs & Riz & $\begin{array}{c}\text { Huile } \\
\text { de soja }\end{array}$ & $\begin{array}{c}\text { Autres } \\
\text { huiles } \\
\text { végé- } \\
\text { tales }\end{array}$ & Sucre & Viande & $\begin{array}{l}\text { Produits } \\
\text { laitiers }\end{array}$ \\
\hline Argentine & 33 & 30 & 3 & 2 & 2 & 7 & 13 & 16 & 8 \\
\hline Brésil & 21 & 13 & 7 & 13 & 11 & $<0.5$ & 13 & 12 & 6 \\
\hline Chili & 23 & 30 & 5 & 3 & 6 & 5 & 14 & 13 & 5 \\
\hline Chine & 40 & 16 & 4 & 27 & 3 & 3 & 2 & 15 & 1 \\
\hline Inde & 50 & 21 & 2 & 30 & 2 & 7 & 7 & $<0.5$ & 6 \\
\hline Indonésie & 48 & 6 & 7 & 51 & 1 & 6 & 6 & $<0.5$ & $<0.5$ \\
\hline Russie & 33 & 33 & $<0.5$ & 2 & $<0.5$ & 7 & 12 & 7 & 11 \\
\hline $\begin{array}{l}\text { Afrique } \\
\text { du Sud }\end{array}$ & 25 & 16 & 31 & 5 & 3 & 7 & 11 & 7 & 2 \\
\hline Ukraine & 61 & 31 & 2 & 1 & $<0.5$ & 8 & 13 & 5 & 10 \\
\hline Vietnam & 51 & 3 & 3 & 62 & 1 & 1 & 5 & 10 & $<0.5$ \\
\hline
\end{tabular}

1. La consommation d'énergie alimentaire par personne correspond à la quantité de nourriture dont dispose chacun des membres de la population totale, exprimée en kcal par personne et par jour : une kcal est égale à 1000 calories. La consommation alimentaire correspond quant à elle à la quantité de nourriture disponible directement destinée à la consommation humaine, telle qu'elle est estimée dans les Bilans alimentaires de la FAO. Elle ne tient pas compte des approvisionnements utilisés pour l'alimentation des animaux.

2. Y compris l'huile de tournesol, l'huile de colza et de moutarde, l'huile de palme, I'huile d'arachide, I'huile de son de riz, I'huile de coco, et l'huile de germes de maïs.

Source: FAO, Statistiques de sécurité alimentaire, 2010.

L'agriculture a un bien plus grande poids dans l'économie des quatre pays d'Asie : elle y contribue en moyenne pour environ $15 \%$ au PIB et emploie aux alentours de $50 \%$ de la population active (graphique 1.9). Par contre, en Afrique du Sud et en Argentine, moins de 10\% de la population participe aux activités agricoles. En Afrique du Sud et au Chili, la part de l'agriculture dans le PIB est inférieure à $5 \%$, niveau tout à fait comparable à celui observé dans bon nombre de pays de l'OCDE. Tous les pays ont certes été confrontés au même dilemme face à la hausse des cours internationaux, puisque les interventions sur le marché visant à faire baisser les prix au profit des consommateurs ont pour effet de pénaliser les producteurs agricoles, mais il se pose avec plus d'acuité aux quatre pays d'Asie, eu égard à la place de l'alimentation dans la consommation des ménages et au poids de l'agriculture dans leur économie. C'est pourquoi ils s'efforcent tout à la fois de protéger les consommateurs de la hausse des prix et de soutenir les producteurs en augmentant les subventions aux intrants. 
Graphique 1.9. Contribution de l'agriculture au PIB et à l'emploi, 2005

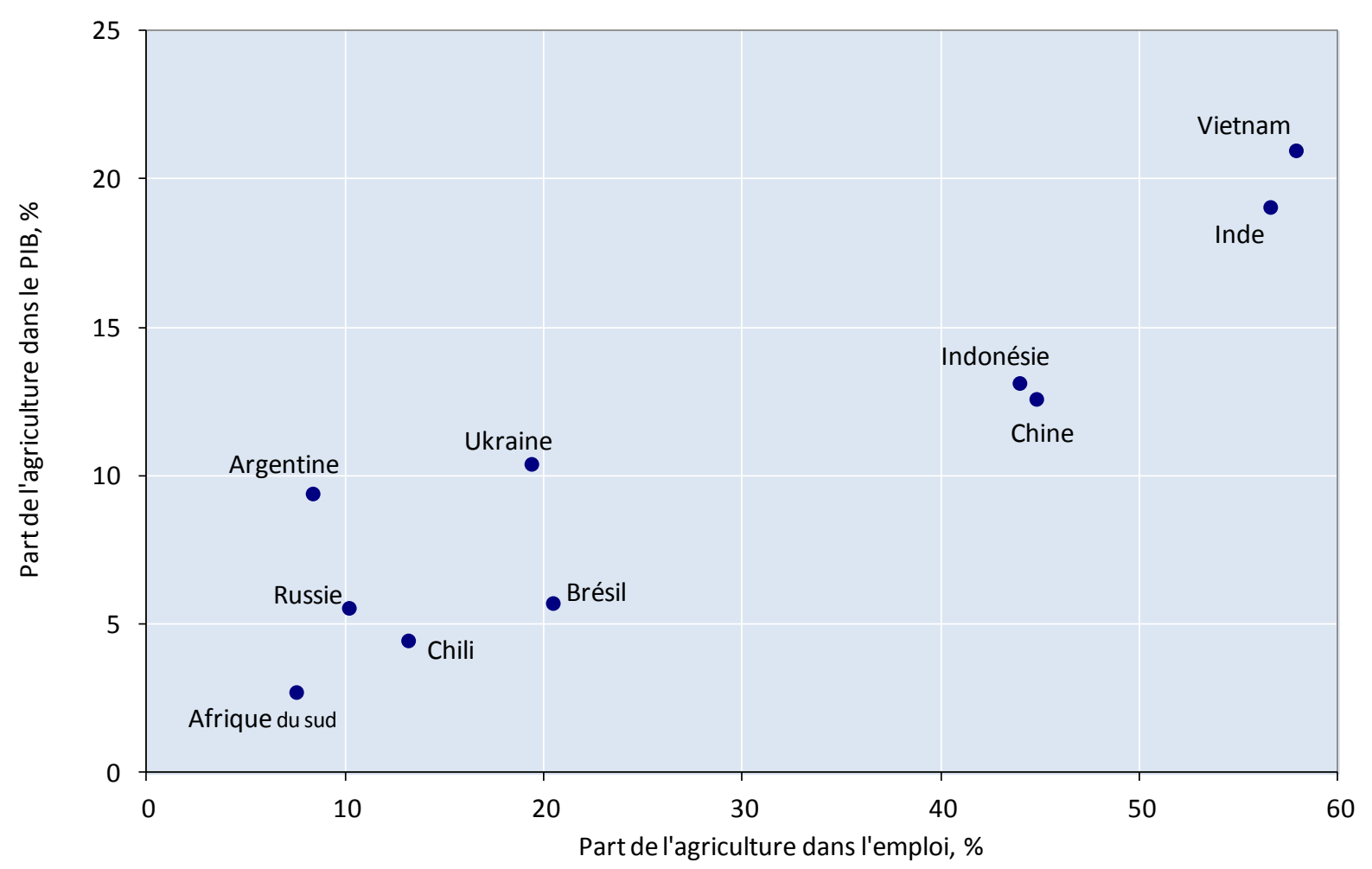

Source : Banque mondiale, Key Development Data and Statistics, 2010 ; Organisation internationale du travail, LABORSTA Internet, 2010 ; Institut national argentin des statistiques et des recensements (INDEC), 2010 ; et OCDE (2009a).

Les pouvoirs publics sont également sensibles aux réactions de l'opinion publique et des médias, ou s'attachent à attirer leur attention et à gagner leur adhésion. Tout porte à croire qu'il est essentiel d'un point de vue politique de montrer que l'on fait quelque chose, surtout dans les pays où des élections doivent avoir lieu sous peu, comme dans le cas de l'Inde ou de l'Ukraine. En Inde, «les politiciens du pays ont continué de se livrer sans la moindre retenue à des calculs électoraux internes au lieu de mettre en œuvre une politique d'alimentation appropriée... Les autorités indiennes - au niveau des États comme à l'échelon national - se sont lancées dans une surenchère pour s'assurer les voix des pauvres en fournissant des céréales à des prix de plus en plus subventionnés » (Slayton, 2009). Cela semble avoir abouti aux plus mauvais choix quant aux types d'interventions.

La position commerciale nette d'un pays, d'un point de vue global comme en ce qui concerne les divers produits considérés séparément, permet dans une large mesure de comprendre les raisons qui poussent les pouvoirs publics à agir face aux hausses des cours internationaux, ainsi que le type de mesures auxquels il peut décider d'avoir recours. Les dix pays présentent des différences du point de vue du rôle des échanges des produits de base examinés (graphique 1.10). Ces différences concernent aussi bien le marché intérieur, c'est-à-dire la part des importations dans la consommation intérieure ou la proportion de la production intérieure qui est exportée (mesurées sur l'axe vertical), que les marchés internationaux, autrement dit les importations ou les exportations en pourcentage des échanges mondiaux (sur l'axe horizontal). Les pays situés dans la partie droite du graphique correspondant à un produit donné sont exportateurs nets de ce produit ; ceux qui apparaissent dans la partie gauche sont au contraire importateurs nets. Pour déterminer si un pays doit être considéré comme exportateur net ou comme importateur net d'un produit donné, on compare le volume moyen de ses exportations et de ses importations de ce produit sur trois ans. Si la moyenne triennale des exportations est supérieure celle des importations, le pays est considéré comme un exportateur net : dans le cas contraire, il est réputé être importateur net. 
Lorsque le pays est exportateur net d'un produit donné, sa part dans les échanges mondiaux est calculée sur la base du volume de ses exportations et la part des échanges sur son marché intérieur correspond au pourcentage de la production qui est exportée. Lorsqu'il est importateur net, sa part dans les échanges mondiaux est calculée sur la base du volume de ses importations et la part des échanges sur son marché intérieur correspond au pourcentage de la consommation intérieure (calculée en additionnant les importations à la production, diminuée des exportations) satisfaite par les importations. Plus un pays est situé vers le haut du graphique correspondant à un produit donné, plus la part des échanges sur son marché intérieur est importante pour ce produit particulier-que ce soit en tant que moyen de satisfaire la consommation si le pays est importateur net ou en tant que débouché pour la production s'il est exportateur net. Plus un pays est éloigné vers la gauche vers ou la droite de la ligne verticale correspondant à $0 \%$ sur l'axe horizontal, plus grande est sa part dans les échanges sur le marché international de ce produit - en tant qu'importateur ou exportateur de ce produit.

Graphique 1.10. Volume des échanges en pourcentage du marché intérieur et du marché international, par produit, 2003-05
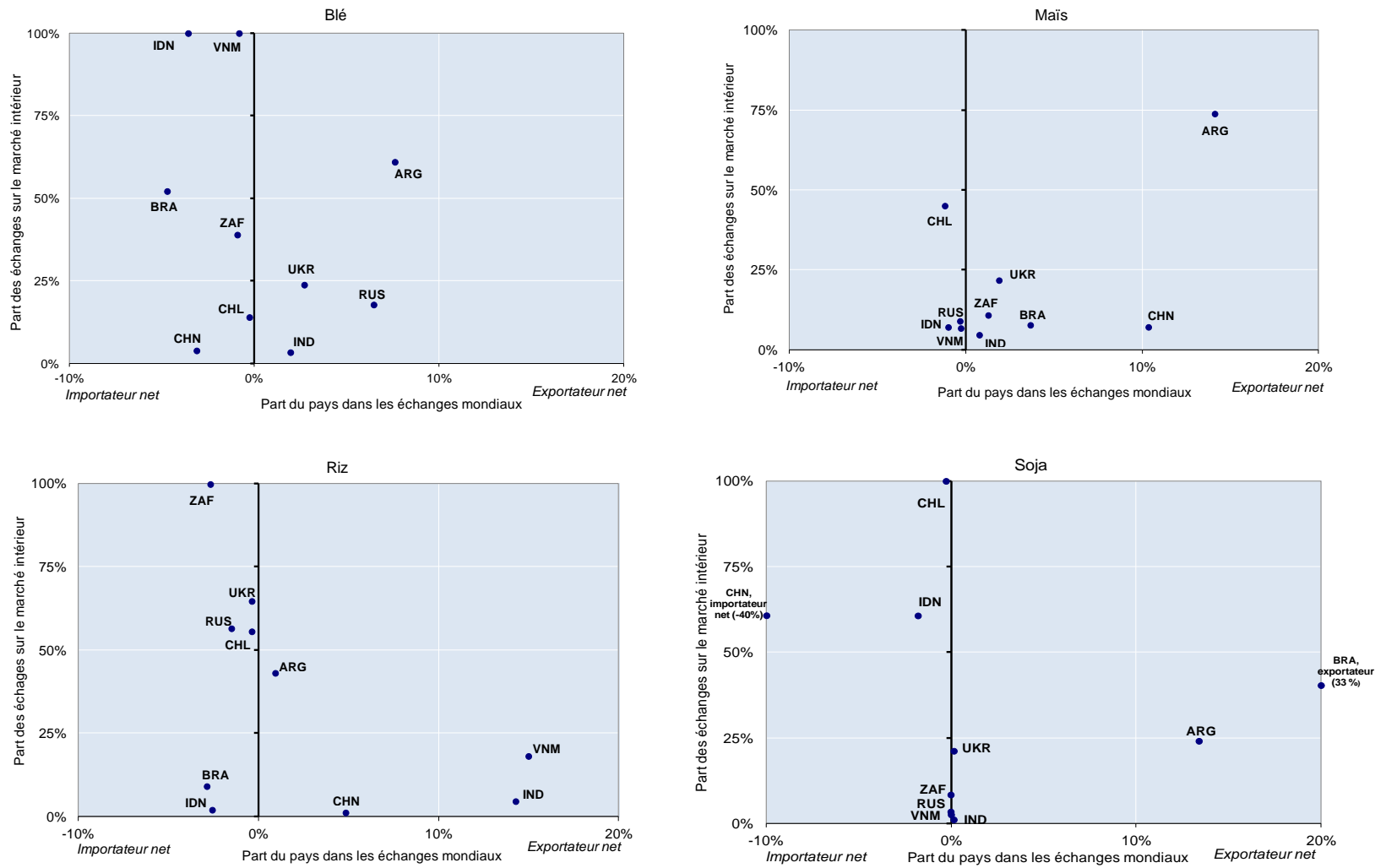

Note : ARG : Argentine ; BRA : Brésil, CHL : Chili, CHN : Chine ; IDN : Indonésie, IND : Inde, RUS : Russie, UKR : Ukraine, VNM : Vietnam, ZAF : Afrique du Sud.

Source : FAO, Base de données FAOSTAT, 2009

Par exemple, l'Argentine, l'Inde, la Russie et l'Ukraine figuraient toutes parmi les exportateurs nets de blé au cours de la période 2003-05 tandis que les six autres pays étaient tous importateurs nets de ce produit. L'Argentine et la Russie exportaient des quantités relativement comparables de blé, à savoir 8.8 million et 7.5 million de tonnes respectivement, soit $7.6 \%$ et $6.5 \%$ des échanges mondiaux, mais ce volume représentait un peu plus de $60 \%$ de la production en Argentine contre moins de $20 \%$ en Russie. L'Indonésie et la Chine ont quant à elles importé des quantités comparables de blé au cours de la période 2003-05, à savoir 4.2 et 3.6 millions de tonnes respectivement, soit $3.6 \%$ et $3.1 \%$ des échanges mondiaux. Ces importations fournissaient la totalité du blé consommé en Indonésie, contre seulement $4 \%$ en Chine. 
Le type de mesures prises est également fonction du contexte. Par exemple, pour baisser les droits de douane et la TVA, encore faut-il que ces prélèvements soient déjà appliqués, et une telle mesure n'aura d'effet que s'ils sont sensiblement réduits. Les réductions des droits de douanes ont joué un rôle plus important en Chine, en Inde et au Vietnam, qui comptaient toutefois parmi les rares pays à mettre en œuvre un niveau de protection relativement élevé pour les produits considérés (graphique 1.11).

Graphique 1.11. Profil tarifaire pour certains produits, 2005
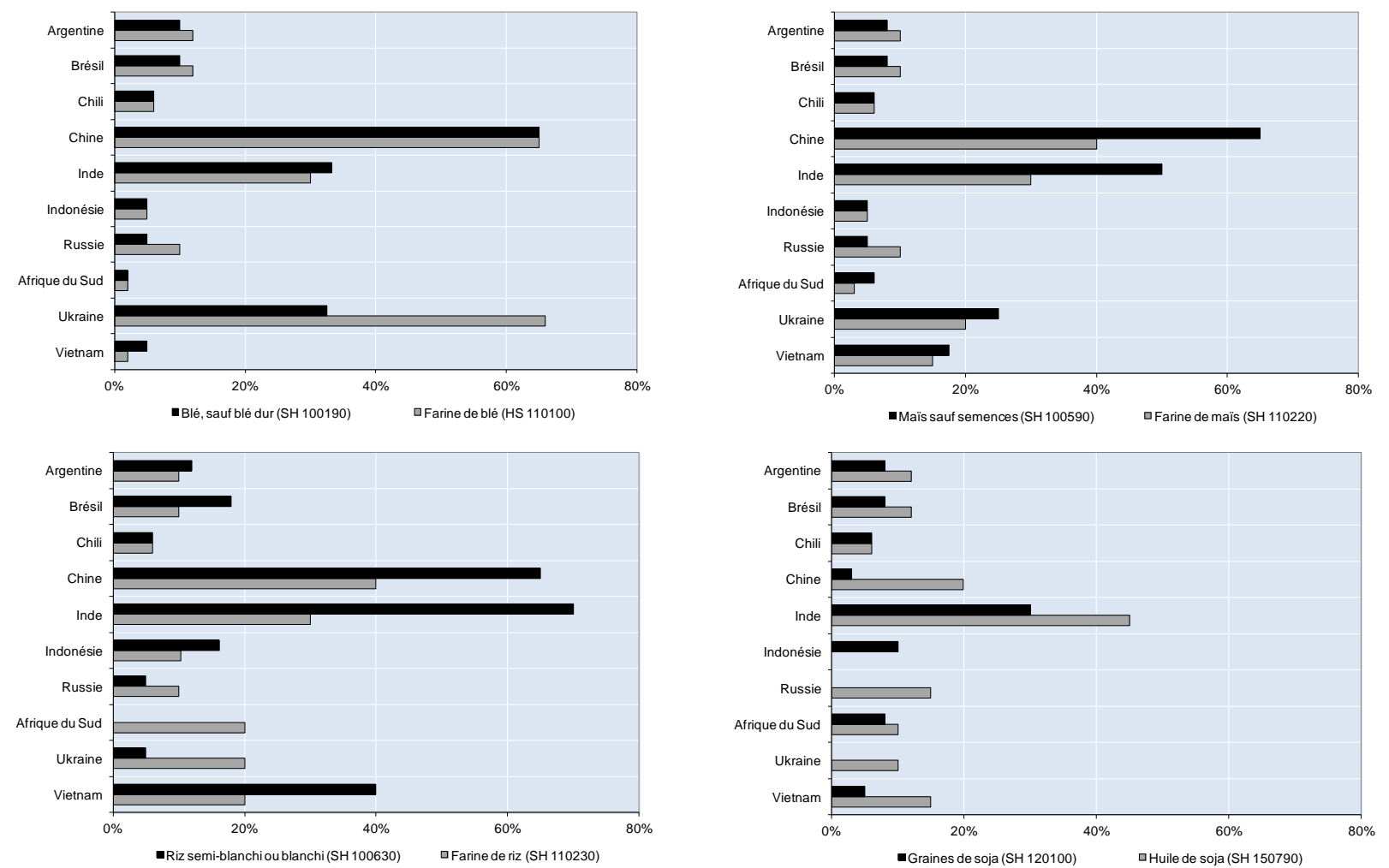

Notes : Les profils tarifaires correspondent aux taux de droits maximaux appliqués à la NPF, sauf dans le cas du Vietnam, où les tarifs consolidés ont été utilisés à la place. Des régimes tarifaires plus favorables que ceux appliqués à la NPF peuvent être accordés en vertu d'accords commerciaux régionaux (ACR) ou de l'octroi de préférences non réciproques.

Les droits spécifiques appliqués en Indonésie sur le riz et la farine de riz ; en Afrique du Sud sur le blé, la farine de blé, le maïs et la farine de maïs ; et en Ukraine sur le blé et la farine de blé, sont convertis en équivalents ad valorem sur la base des statistiques commerciales annuelles issues de la base de données Comtrade de l'ONU.

Source : OMC, Fonction de téléchargement des données tarifaires, 2010 ; ONU, Base de données Comtrade, 2010.

Les filets de sécurité sont plébiscités en tant que meilleur moyen de protéger les consommateurs les plus vulnérables, par opposition à des instruments d'action moins ciblés tels que les droits de douane et les mesures de restriction des exportations. Ils permettent en effet de soutenir le pouvoir d'achat des pauvres sans fausser les incitations à produire davantage de nourriture au sein du pays, ni réduire les revenus des vendeurs de produits alimentaires en situation de pauvreté. Ils nécessitent toutefois à l'évidence une augmentation des dépenses publiques. Les capacités de compensation du coût croissant des produits alimentaires pour les consommateurs dépendent tout à la fois des ressources budgétaires disponibles et de l'importance de la consommation alimentaire des ménages. Le graphique 1.12 montre le rapport entre les recettes budgétaires publiques et les dépenses de consommation alimentaire des ménages. 


\section{Graphique 1.12. Capacités budgétaires de compensation du coût accru de la consommation alimentaire des}

ménages, 2003-05

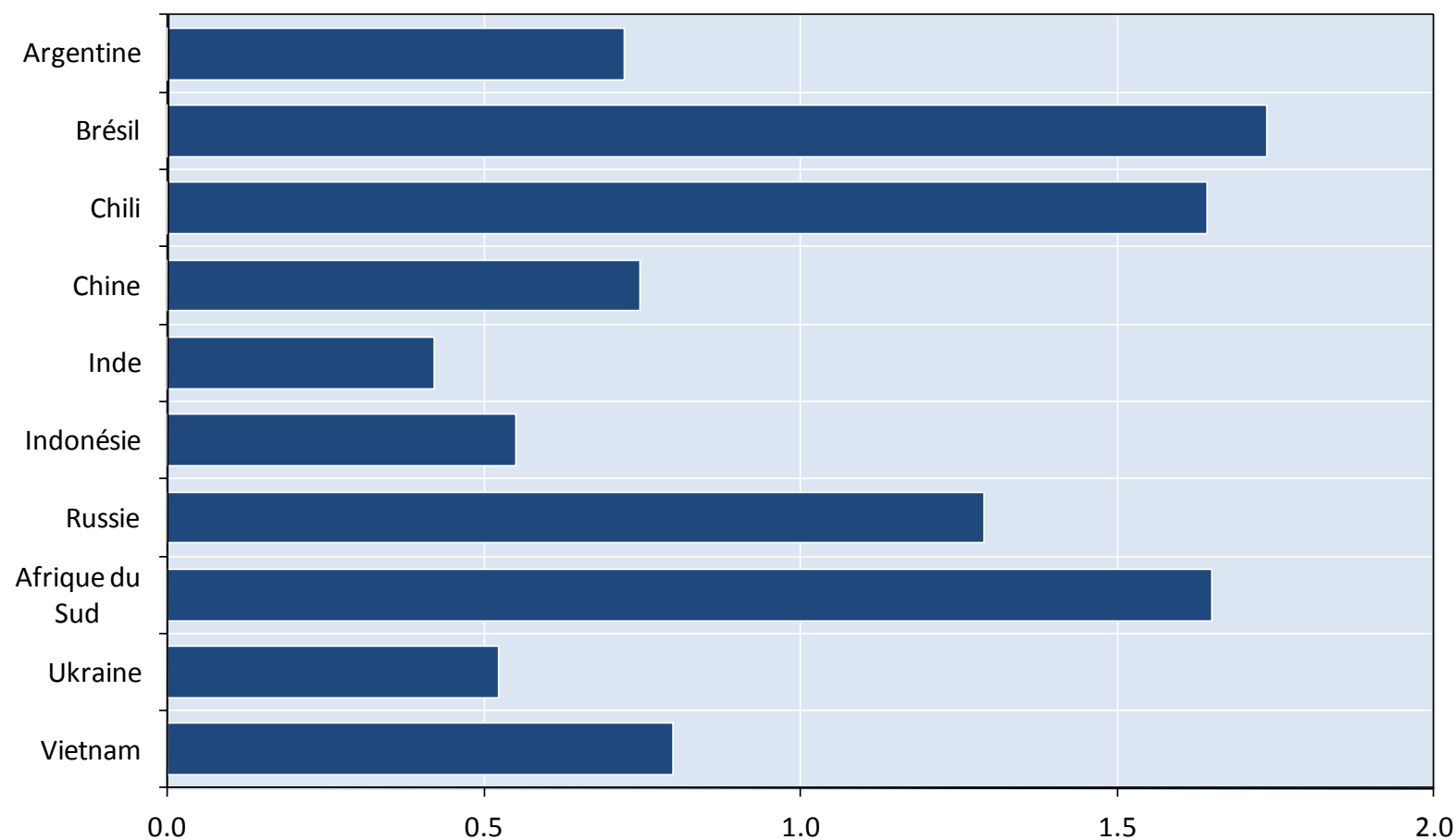

Rapport entre les recettes budgétaires publiques et la consommation alimentaire des ménages

Notes: Les dépenses alimentaires nationales sont estimées en multipliant les dépenses de consommation des ménages à l'échelle nationale, telles qu'elles ressortent des calculs du PIB, par la part de l'alimentation dans les dépenses de consommation des ménages, telle qu'elle est mesurée dans les enquêtes nationales.

Pour l'Argentine : 2002-04.

Source : FMI, Statistique financières internationales, 2010 et FAO, Statistiques de sécurité alimentaire, 2010.

Plus ce rapport sera élevé, plus grand sera le pourcentage de la consommation des ménages qui pourra être financée par une même augmentation en pourcentage des recettes budgétaires. Par exemple, un rapport de 1.5 indique que le transfert aux consommateurs de $1 \%$ des recettes budgétaires leur procurera l'équivalent de $1.5 \%$ de la consommation alimentaire des ménages. À l'inverse, un rapport de 0.5 indique que le transfert aux consommateurs de $1 \%$ des recettes budgétaires ne leur procurera que l'équivalent de $0.5 \%$ de la consommation alimentaire des ménages. Dans plus de la moitié des pays, ce rapport est inférieur à un. Les pays où ce rapport atteint les niveaux les plus élevés, à savoir l'Afrique du Sud, le Brésil et le Chili, ont tous pris des mesures pour protéger les consommateurs. Ils bénéficient donc d'un double avantage : non seulement ils bénéficient de la mise en œuvre de ces mesures mais en outre celles-ci leur imposent un moindre coût budgétaire. 


\section{2. Évaluation de l'impact sur le marché intérieur des mesures à court terme prises par les pouvoirs publics}

Cette section vise à évaluer l'efficacité des mesures à court terme prises par les pouvoirs publics pour répondre à la hausse des cours mondiaux, eu égard à leurs objectifs concernant le marché intérieur. Divers indicateurs sont utilisés pour examiner les évolutions qui affectent les flux commerciaux, la transmission des prix, l'inflation, la consommation et la production au niveau national. ${ }^{4}$ L'évaluation des impacts sur les ménages ne peut être envisagée, du fait des données qui seraient nécessaires, par exemple sur l'état nutritionnel des populations vulnérables et leurs habitudes de consommation. Les mesures prises par les dix pays examinés est d'une telle diversité que l'évaluation de leurs impacts pose des problèmes considérables. Une autre difficulté tient au fait que plusieurs mesures peuvent être appliquées simultanément, empêchant d'isoler les effets de chacune d'elles. L'évaluation est rendue encore plus complexe par la crise financière mondiale qui a débuté à la mi-2008 et par le ralentissement de l'économie qui s'en est suivi. La situation du marché s'en est trouvée totalement bouleversée. Les cours internationaux des produits de base ont fortement chuté et le dollar des États-Unis s'est apprécié par rapport à la plupart des autres monnaies.

Pour prendre toute la mesure des effets de l'action des pouvoirs publics, il faut élaborer un scénario contrefactuel (de référence) (Benson et al., 2008). Il devrait en théorie correspondre à la situation qui se serait produite en l'absence d'intervention des pouvoirs publics. Toute la difficulté tient au fait que cette situation contrefactuelle ne peut par définition être observée. Pour remédier à ce problème, l'étude a recours à trois méthodes couramment utilisées. Elle compare tout d'abord la situation qui résulte de la mise en œuvre de la mesure considérée à celle observée avant son adoption (comparaison «avant-après »). L'étude porte essentiellement sur la période triennale allant de la mi-2006 à la mi-2009. Des comparaisons sont par conséquent effectuées avec la situation au cours de la précédente période triennale, de la mi-2003 à la mi-2006. Pour tenir compte de l'impact du ralentissement économique mondial, l'étude distingue les évolutions intervenues depuis la mi-2006 jusqu'à la mi-2008 de celles qui se sont produites entre la mi2008 et la mi-2009.

L'étude compare également les évolutions observées selon les pays, ce qui constitue une forme de comparaison «avec-sans ». Comme indiqué dans la section précédente, les mesures prises par les pouvoirs publics dans chacun des dix pays examinés ont été très variables, l'Afrique du Sud et le Chili se montrant bien moins enclins à intervenir sur le marché agricole que les huit autres pays. Toutefois, la plus grande prudence s'impose lorsqu'il s'agit de comparer de petites économies relativement ouvertes à de grands pays tels que la Chine et l'Inde, où l'État conserve un rôle important dans le secteur agricole. L'étude procède en outre à des comparaisons avec un pays «tiers » tel que les États-Unis (échanges et production), avec un indice des prix internationaux (inflation), avec la zone de l'OCDE (inflation et consommation), ou encore avec l'ensemble du monde (production).

Pour finir, l'étude compare également les évolutions selon les différents produits, ce qui constitue une autre forme de comparaison «avec-sans ». Elle est axée sur quatre cultures - blé, maïs, riz et soja. Les trois céréales ont connu une forte augmentation de leurs cours internationaux; elles occupent une place relativement importante dans la consommation, surtout pour les couches les plus pauvres de la population ;

4. Bien que les flux commerciaux soient influencés par les prix, la production et la consommation, l'évaluation est réalisée dans cet ordre du fait qu'un certain nombre de pays ont tenté de limiter la transmission des cours internationaux sur leur marché intérieur en ayant recours à des mesures de politique commerciale telles que celles de restriction des exportations. Lorsque tel est le cas, il est peut-être préférable de considérer les flux commerciaux comme un facteur ayant une incidence sur les prix intérieurs plutôt que comme le résultat résiduel d'un arbitrage entre les prix intérieurs et les cours internationaux sur un marché parfaitement concurrentiel. 
et elles ont le plus souvent été directement visées par les mesures prises par les pouvoirs publics. Le soja a été choisi à titre comparatif car les cours internationaux de ce produit de base ont augmenté tout aussi vite que ceux des trois céréales, sans susciter pour autant le même degré d'intervention des pouvoirs publics du fait qu'il n'occupe pas une place aussi importante dans la consommation. Qui plus est, en bien des endroits de la planète, le soja est en concurrence directe avec les céréales à l'heure du choix des cultures par les exploitants.

Il importe de tenir compte du déroulement dans le temps et de l'ampleur des hausses et des baisses des cours de ces produits lors de l'évaluation des mesures prises par les pouvoirs publics (tableau 2.1). S'il est relativement aisé d'observer le mois où les cours ont atteint leur niveau maximal, il peut être difficile de déterminer le mois précis où ils ont commencé à augmenter. D'importantes différences peuvent être observées concernant le déroulement dans le temps et l'ampleur de la hausse.

Tableau 2.1. Déroulement dans le temps et ampleur des variations des cours internationaux du blé, du maïs, du riz et du soja

\begin{tabular}{l|cccc}
\hline & Blé & Maïs & Riz & Soja \\
\hline $\begin{array}{l}\text { Mois où les cours ont commencé à } \\
\text { augmenter }\end{array}$ & Juin 2007 & Octobre 2006 & Novembre 2007 & Octobre 2006 \\
$\begin{array}{l}\text { Mois où les cours ont atteint leur } \\
\text { niveau maximal }\end{array}$ & Mars 2008 & Juin 2008 & Mai 2008 & Juillet 2008 \\
$\begin{array}{l}\% \text { de hausse du cours mensuel } \\
\text { moyen }\end{array}$ & $137 \%$ & $137 \%$ & $185 \%$ & $163 \%$
\end{tabular}

Mois où les cours ont atteint le creux de la vague

$\%$ de baisse du cours mensuel moyen $^{2}$

$\%$ d'augmentation du cours mensuel moyen entre la mi-2006 et la mi- $2009^{3}$

Décembre 2008 Décembre 2008 Décembre 2008 Décembre 2008

Blé : blé dur rouge d'hiver $n^{\circ} 2$ des États-Unis, f.à.b. Golfe du Mexique $\quad$ Maïs : maïs jaune $n^{\circ} 2$ des États-Unis, f.à.b.

Riz : riz blanc thaïlandais de second choix $100 \%$ B, f.à.b. Bangkok Golfe du Mexique

Soja : soja jaune $n^{\circ} 1$ des États-Unis, f.à.b.

Golfe du Mexique

1. Mesuré du mois précédent celui où les cours ont commencé à augmenter jusqu'au mois où ils ont atteint leur niveau maximal.

2. Mesuré du mois où les cours ont atteint leur niveau maximal jusqu'à celui où ils sont tombés à leur point le plus bas.

3. Sur la base des cours mensuels moyens au deuxième trimestre, couvrant la période d'avril à juin, sauf dans le cas du blé, où c'est le premier trimestre, de janvier à mars, qui est utilisé afin de permettre les comparaisons. Ces trimestres ont été retenus afin de rendre ce tableau directement comparable avec l'analyse de la transmission des prix qui figure à la section 2.2.

Source : FAO, Prix internationaux des produits de base, 2010, www.fao.org/es/esc/prices/PricesServlet.jsp?lang=fr.

Les cours internationaux du maïs et du soja ont commencé à augmenter plus tôt que ceux du blé et du riz, mais ce sont ceux du riz qui ont enregistré la hausse la plus forte et la plus rapide. Le déroulement dans le temps et l'ampleur de la baisse des cours qui a suivi ont également été variables selon les produits. Pour l'ensemble des quatre produits de base, les cours internationaux ont atteint leur plus bas niveau en décembre 2008. À la mi-2009, les cours étaient encore plus de $40 \%$ plus élevés que trois ans plus tôt, et ils restaient même dans certains cas près de deux fois supérieurs à leur niveau de l'époque. L'annexe $\mathrm{B}$ offre davantage de précisions sur les variations des cours internationaux de ces produits de base ainsi que de certains autres au long de cette période.

\subsection{Flux commerciaux}

Comme cela a été mentionné à la section 1 , bon nombre de pays ont réagi à la hausse des cours internationaux des produits de base par l'instauration de mesures commerciales ou par le réajustement de celles déjà en place. Une disposition fréquente a consisté à réduire les droits de douane à l'importation, et certains pays ont en outre accru les contingents d'importation ou réduit les exigences auxquelles doivent se 
conformer les importations. Par ailleurs, quelques pays en nombre plus restreint ont eu recours à des mesures de restriction des exportations telles que des taxes, des prix minimums à l'exportation, des contingents, des systèmes de licence, ou encore des interdictions pures et simples. L'étude de l'évolution de la structure des échanges peut permettre d'évaluer l'efficacité de ces mesures au regard de leur impact direct et immédiat sur les flux commerciaux.

Les graphiques $2.1,2.4,2.6$ et 2.8 montrent les variations annuelles des volumes des échanges pour les quatre produits de base considérés. Les pays sont classés dans deux groupes différents selon qu'ils étaient exportateurs nets ou importateurs nets du produit de base considéré durant la période triennale de référence (depuis la campagne de commercialisation s'achevant en 2004 jusqu'à la campagne de commercialisation s'achevant en 2006). Pour les importateurs nets, ce sont les variations du volume des importations qui sont présentées. Pour les exportateurs nets, ce sont celles du volume des exportations. Les graphiques 2.2, 2.5, 2.7 et 2.9 portent plus particulièrement sur les évolutions des échanges de certains des pays exportateurs qui ont imposé des mesures de restriction des exportations pendant la période examinée. Ils indiquent les variations trimestrielles des quantités exportées par ces pays. Pour tenir compte des variations saisonnières, chaque trimestre est indexé sur le niveau moyen des exportations au cours du trimestre correspondant de la période triennale de référence.

Les importateurs nets de blé ont pu importer des volumes similaires ou supérieurs à ceux du passé (graphique 2.1). Cela s'avère particulièrement important pour l'Indonésie et le Vietnam, qui ne produisent pas du tout de blé. Pour les exportateurs nets, les volumes annuels exportés ont sensiblement diminué en Inde et en Ukraine (2006/07 et 2007/08), et dans une moindre mesure en Argentine. Du fait des mesures de restriction, les exportations de blé de l'Argentine, de la Chine, de la Russie et de l'Ukraine ont atteint leur plus faible niveau au cours du deuxième trimestre 2008 , juste après que les cours internationaux ont atteint leur niveau maximal en mars 2008.

Les contingents d'exportation imposés par l'Ukraine ont fortement limité les exportations de blé d'octobre 2006 à mai 2008. Lorsque le contingent a brièvement été levé entre la mi-mai et la fin juin 2007, chacun s'est rué pour vendre ses produits, de sorte que les exportations réalisées en juin ont représenté $20 \%$ du volume annuel total de 2006/07. La taxe sur les exportations de blé imposée en Russie n'a pas eu d'effet notable sur les volumes trimestriels exportés jusqu'à ce que les cours internationaux du blé commencent à diminuer début 2008. Prévenus que cette taxe à l'exportation serait appliquée à partir du 12 novembre 2008 (qui avait été officiellement annoncée un mois auparavant), les exportateurs ont exporté autant de blé qu'il leur était possible avant cette date. Le volume annuel n'a pas diminué, mais la taxe à l'exportation a modifié l'échelonnement dans le temps des exportations de blé de la Russie. Les volumes exportés par la Russie et l'Ukraine ont sensiblement augmenté après la levée des mesures de restriction des exportations en juin 2008, liée aux récoltes exceptionnelles de 2008. 


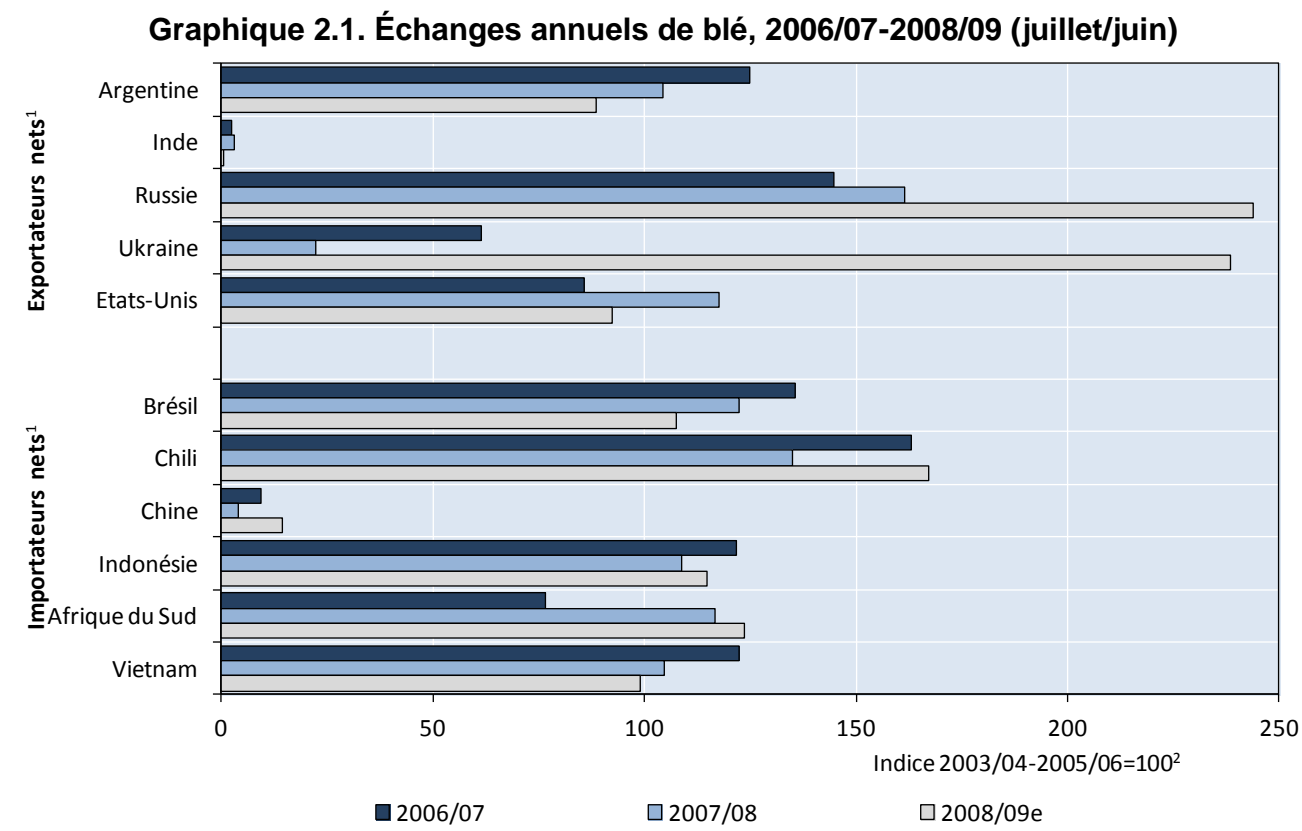

e : estimation.

1. Pour déterminer si un pays doit être considéré comme exportateur ou importateur net de blé, on compare le volume moyen de ses exportations et de ses importations de ce produit au cours de la période triennale indiciaire 2003/04-2005/06. Le volume des échanges correspond aux exportations dans le cas des exportateurs nets et aux importations dans celui des importateurs nets. Les chiffres indiqués intègrent la farine de blé et le blé dur (grains et semoule).

2. Pour l'Ukraine, l'indice est basé sur la moyenne biennale 2004/05-2005/06 plutôt que sur celle triennale utilisée pour les neuf autres pays, des conditions météorologiques exceptionnellement rigoureuses ayant abouti en 2003/04 à la plus mauvaise récolte de blé jamais enregistrée.

Source : Conseil international des céréales (2010).

Graphique 2.2. Exportations trimestrielles de blé de l'Argentine, de la Chine, de la Russie et de l'Ukraine, 2006/07-2008/09

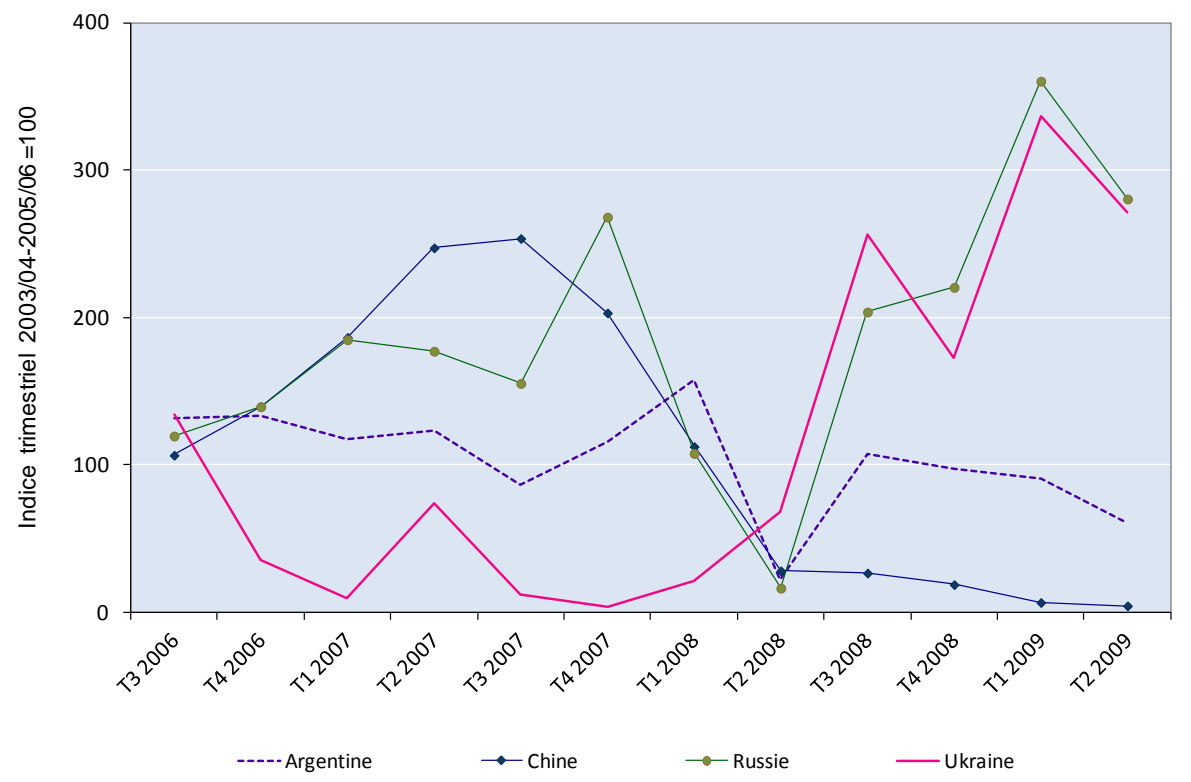

1. Pour l'Ukraine, l'indice est basé sur la moyenne biennale 2004/05-2005/06 plutôt que sur celle triennale utilisée pour l'Argentine et la Russie, des conditions météorologiques exceptionnellement rigoureuses ayant abouti en 2003/04 à la plus mauvaise récolte de blé jamais enregistrée.

2. Pour la Chine, les chiffres trimestriels correspondant à T1 et T2 2009 sont estimées sur la base du volume total 2008/09 et des quantités observées aux trimestres T3 et T4 2008.

Source : Conseil international des céréales (2010). 
Les exportations de blé de l'Argentine, qui avaient été soumises à des mesures de restriction pendant plusieurs années avant la période objet de l'étude, ont eu tendance à diminuer du fait de la baisse de la production. L'augmentation de $20 \%$ à $28 \%$ de la taxe à l'exportation entre novembre 2007 et décembre 2008 n'a pas semblé provoquer une variation notable des volumes exportés, bien qu'elle ait diminué les gains des producteurs de blé par rapport au niveau qu'ils auraient dû atteindre en l'absence de cette mesure, ce qui a contribué à une baisse des superficies plantées pour la récolte de 2008. L'évolution des exportations est fortement influencée par les décisions d'ouvrir ou de clore l'enregistrement des exportations, ainsi que par les volumes admis à l'enregistrement. En particulier, la décision de clore l'enregistrement des exportations de novembre 2007 jusqu'à mai 2008 a abouti à une forte contraction des volumes d'exportation au deuxième trimestre de 2008. Cette décision a été à l'origine prise en raison des craintes initiales quant à l'impact potentiel des fortes gelées survenues dans les principales régions productrices de blé au moment de la récolte, mais elle a été maintenue malgré un impact effectif limité sur les niveaux de production en 2007. Les récoltes de blé extrêmement médiocres obtenues en 2008 du fait de la sécheresse ont été le principal facteur à l'origine de l'effondrement des exportations en 2008/09.

Pendant la période de référence 2004-06, l'Inde a été exportateur net de blé alors que la Chine a été importateur net. La situation s'est inversée durant la période 2007-09 (graphique 2.3). En 2003/04, l'Inde avait exporté 5.4 millions de tonnes de blé. En 2006/07, elle a dû importer 6.7 millions de tonnes à la suite des mauvaises récoltes de 2005 et 2006. Malgré des récoltes exceptionnelles en 2007-09, 1'interdiction des exportations de blé a fait que les échanges commerciaux du pays ont été minimes. En 2004/05, la Chine avait importé 6.6 millions de tonnes de blé ; en 2006/07 et 2007/08, elle en a exporté 2.4 millions de tonnes. Cependant, la suppression des restitutions à l'exportation en décembre 2007, l'imposition de taxes à l'exportation en janvier 2008, et l'octroi limité de contingents d'exportation ont arrêté net cette tendance à l'augmentation des exportations chinoises (graphique 2.2).

Graphique 2.3. Exportations nettes de blé de I'Inde et de la Chine, 2003/04-2008/09 (juillet/juin)

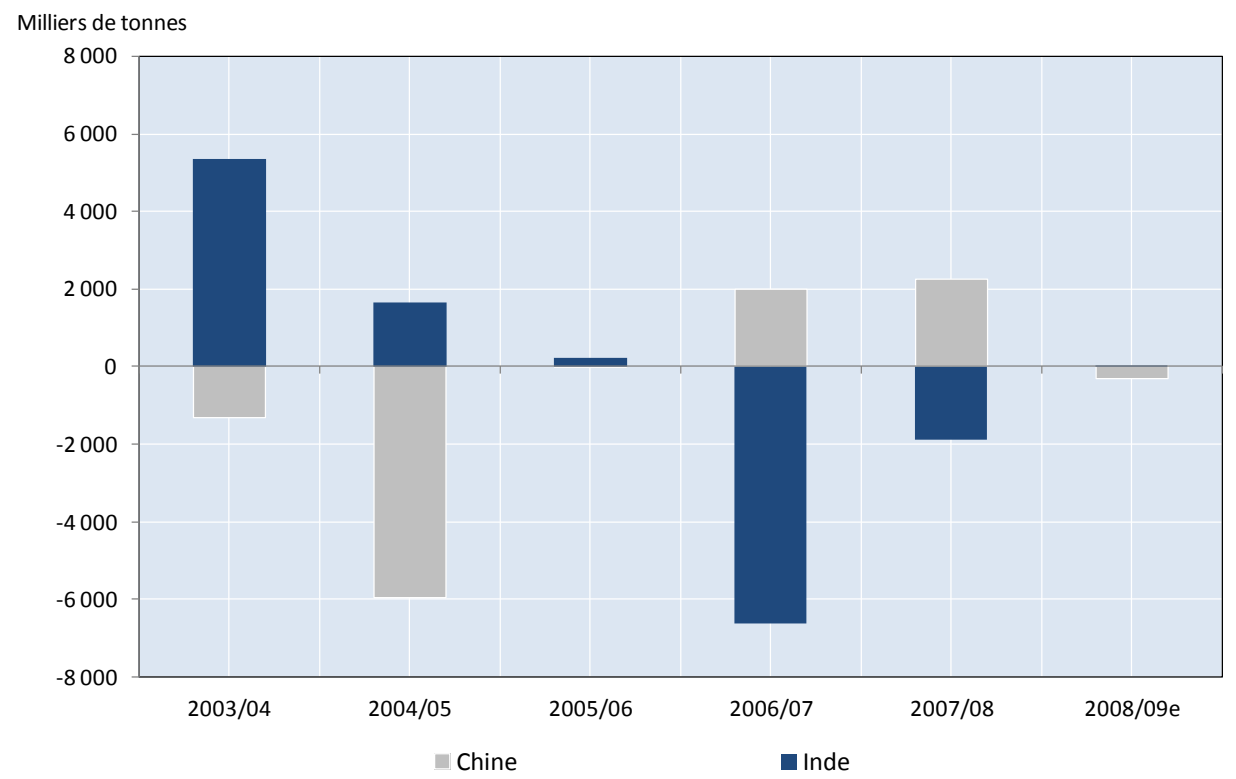

e : estimation.

Source : Conseil international des céréales (2010). 


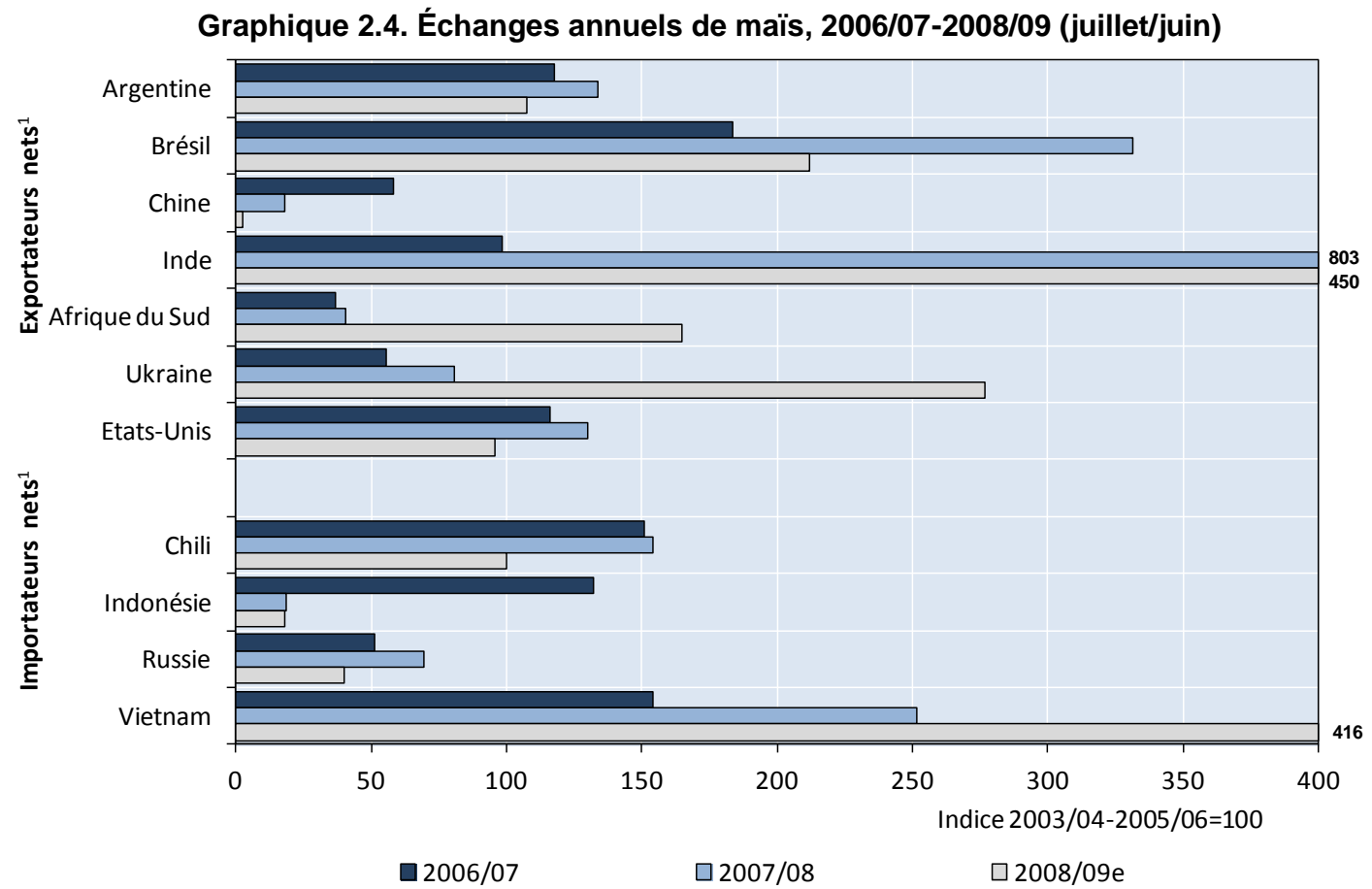

e : estimation.

1. Pour déterminer si un pays doit être considéré comme exportateur ou importateur net de blé, on compare le volume moyen de ses exportations et de ses importations de ce produit au cours de la période triennale indiciaire 2003/04-2005/06. Le volume des échanges correspond aux exportations dans le cas des exportateurs nets et aux importations dans celui des importateurs nets.

Source : Conseil international des céréales (2010).

Graphique 2.5. Exportations trimestrielles de maïs de l'Argentine, de la Chine et de l'Ukraine, 2006/07-2008/09

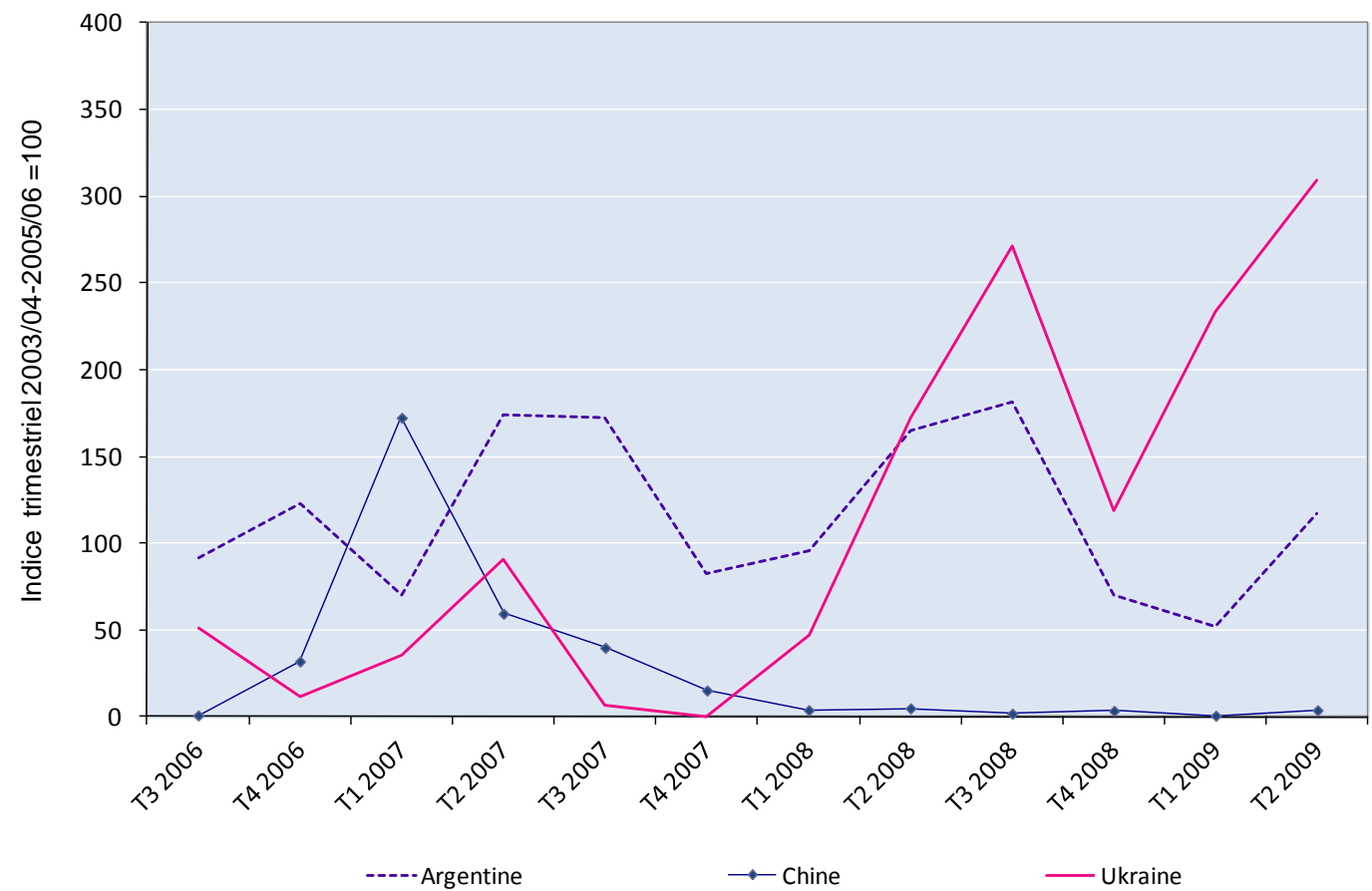

Source : Conseil international des céréales (2010). 
De tous les importateurs nets de maïs (graphique 2.4), le Chili est le plus dépendant à l'égard des importations et il est parvenu à les maintenir à un niveau relativement stable malgré des cours internationaux plus élevés. La forte augmentation des importations de maïs au Vietnam peut être attribuée à la demande du secteur de l'élevage, en pleine expansion. Les importations de maïs ont chuté en Russie et en Indonésie. La hausse des prix a entraîné une expansion de la production dans l'un et l'autre pays, ainsi que la recherche d'autres sources, moins onéreuses, d'alimentation des animaux, telles que la farine de viande et d'os en Indonésie.

L'examen des évolutions annuelles et trimestrielles des exportations dans les différents pays ayant appliqué des mesures de restriction des exportations de maïs permet de tirer à peu près les mêmes conclusions que pour le blé. Les mesures de restriction des exportations appliquées par l'Argentine n'ont rien changé au volume de ses exportations annuelles, mais l'ouverture et la clôture de l'enregistrement en a modifié l'échelonnement dans le temps. Une conclusion similaire se dégage de l'examen des évolutions annuelles et trimestrielles des exportations de soja de l'Argentine (graphiques 2.8 et 2.9). Les contingents d'exportation imposés par l'Ukraine en 2006/07 et 2007/08 ont fortement limité les exportations de maïs. Comme pour le blé, ce n'est que lorsque les contingents d'exportation de maïs ont été provisoirement levés de la fin février jusqu'à la fin juin 2007 que des exportations de quelque importance par rapport aux niveaux enregistrés par le passé ont pu être observées. Les exportations de maïs de la Chine ont chuté en 2007 et étaient quasiment inexistantes en 2008 et 2009, à la suite de diverses mesures de restriction des exportations.

La plupart des importateurs nets de riz ont réduit leurs importations du fait de la hausse des cours mondiaux (graphique 2.6). La forte augmentation des importations de riz survenue en Indonésie en 2006 était nécessaire du fait de la récolte moins bonne que prévue qui a contraint les autorités à importer du riz pour enrayer la flambée des prix intérieurs (graphique 2.6). La baisse des importations de riz qui s'est produite en Russie en 2007-09 par rapport à 2004-06 a été largement due à une augmentation des droits de douane saisonniers et à un durcissement des exigences de certification. Ces dispositions visaient à favoriser une augmentation de la production intérieure de riz.

Malgré l'ajustement des objectifs d'exportation, l'ouverture et la clôture de l'enregistrement des exportations et le durcissement des exigences à respecter, le volume annuel des exportations de riz du Vietnam n'a pas diminué en 2008 par rapport aux niveaux antérieurs. Bien que l'on ne dispose pas de statistiques trimestrielles, il semble probable que l'échelonnement dans le temps de ces exportations ait été gravement perturbé par ces mesures.

Avant l'application des mesures de restriction à l'exportation, les exportations de riz de l'Inde étaient en expansion en 2007. L'interdiction des exportations privées de riz en octobre 2007, et l'instauration de prix minimums à l'exportation (PME) et de taxes à l'exportation de riz basmati en mars-avril 2008 ont entraîné une baisse des exportations de riz. Le retard à supprimer les PME sur le riz basmati a fait perdre aux exportateurs indiens des parts de marché au profit de leurs concurrents pakistanais, dont les PME avaient été abolis fin 2008 et qui avaient bénéficié d'une dépréciation marquée de leur monnaie par rapport à la roupie indienne (Slayton, 2009). La part de l'Inde dans les exportations mondiales de riz est passée de 19\% en 2007 (elle était en moyenne de 15\% au cours de la période 2004-06) à seulement 7\% en 2009.

Contrairement à 1 'Inde, la Chine a continué à exporter du riz et à procéder à de nouvelles ventes en dépit de l'application d'une taxe à l'exportation et de la suppression du remboursement de la TVA, et malgré une légère baisse par rapport à 2006 et 2007. Cependant, lors de la crise du riz au début 2008, la Chine n'a pas répondu aux exhortations publiques et privées pour qu'elle utilise ses excédents croissants de riz pour combler partiellement le vide laissé par la sortie de l'Inde et du Vietnam du marché (Slayton, 2009). La Chine a tardé à octroyer des contingents d'exportation et n'a exporté que 56000 tonnes au plus fort du marché, en avril-juin 2008, contre 167000 tonnes au cours de la même période l'année précédente. 
Graphique 2.6. Échanges annuels de riz, 2006-09

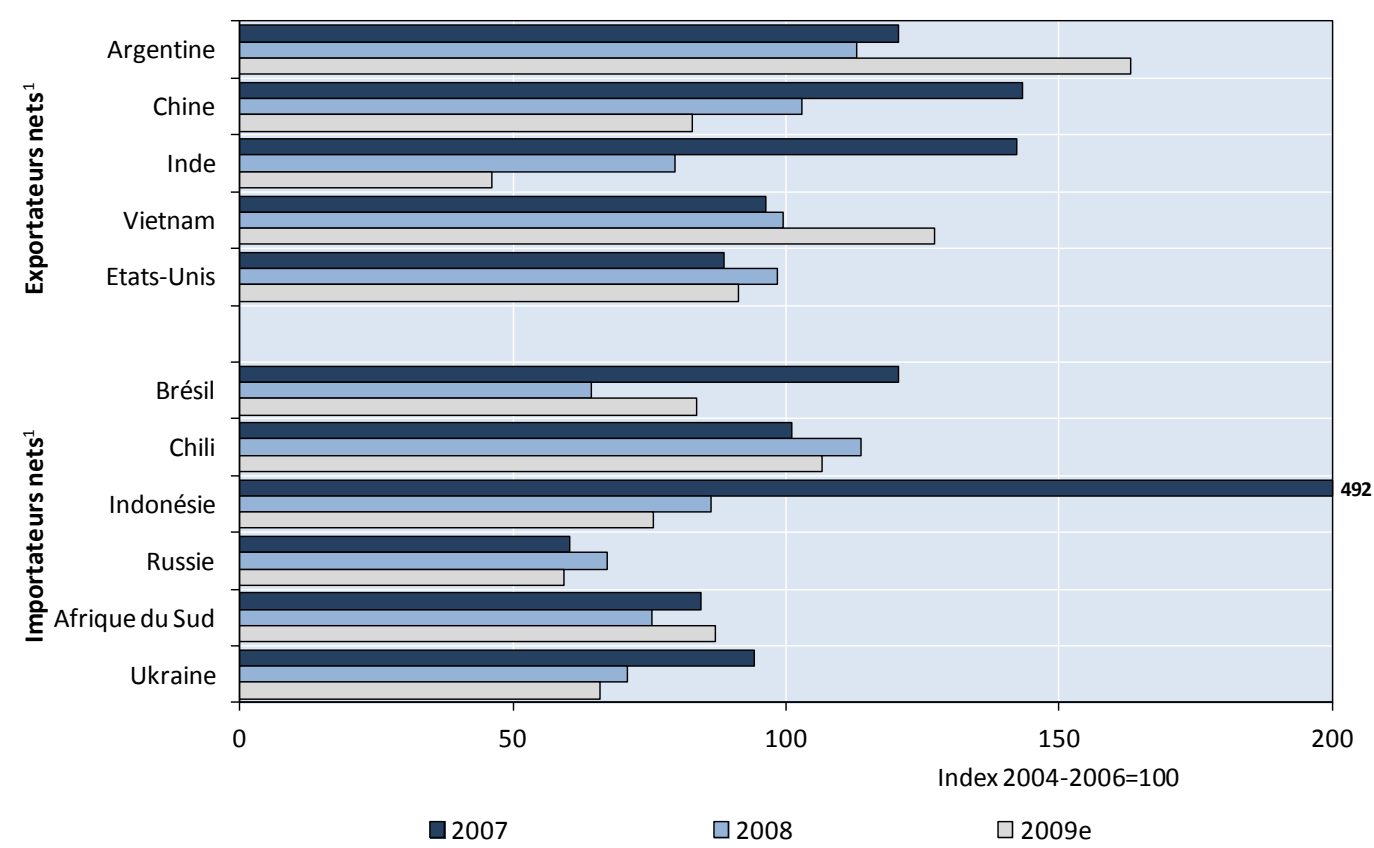

e : estimation.

1. Pour déterminer si un pays doit être considéré comme exportateur ou importateur net de riz, on compare le volume moyen de ses exportations et de ses importations de ce produit au cours de la période triennale indiciaire 2003/04-2005/06. Le volume des échanges correspond aux exportations dans le cas des exportateurs nets et aux importations dans celui des importateurs nets. Source : Conseil international des céréales (2010).

\section{Graphique 2.7. Exportations trimestrielles de riz de la Chine et de l'Inde, 2006/07-2008/09}

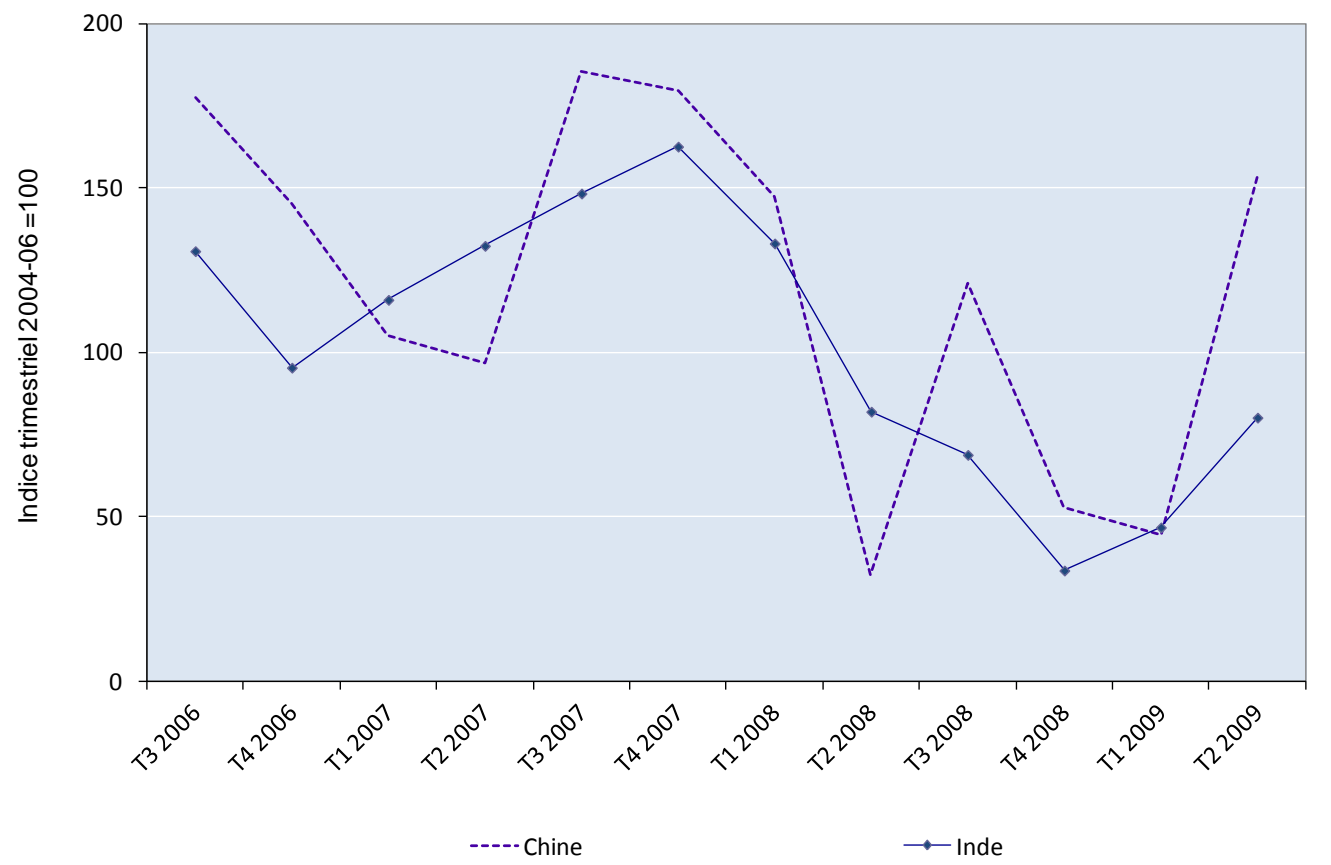

Source : Conseil international des céréales (2010). 


\section{Graphique 2.8. Échanges annuels de soja, 2006/07-2008/09 (octobre/septembre)}

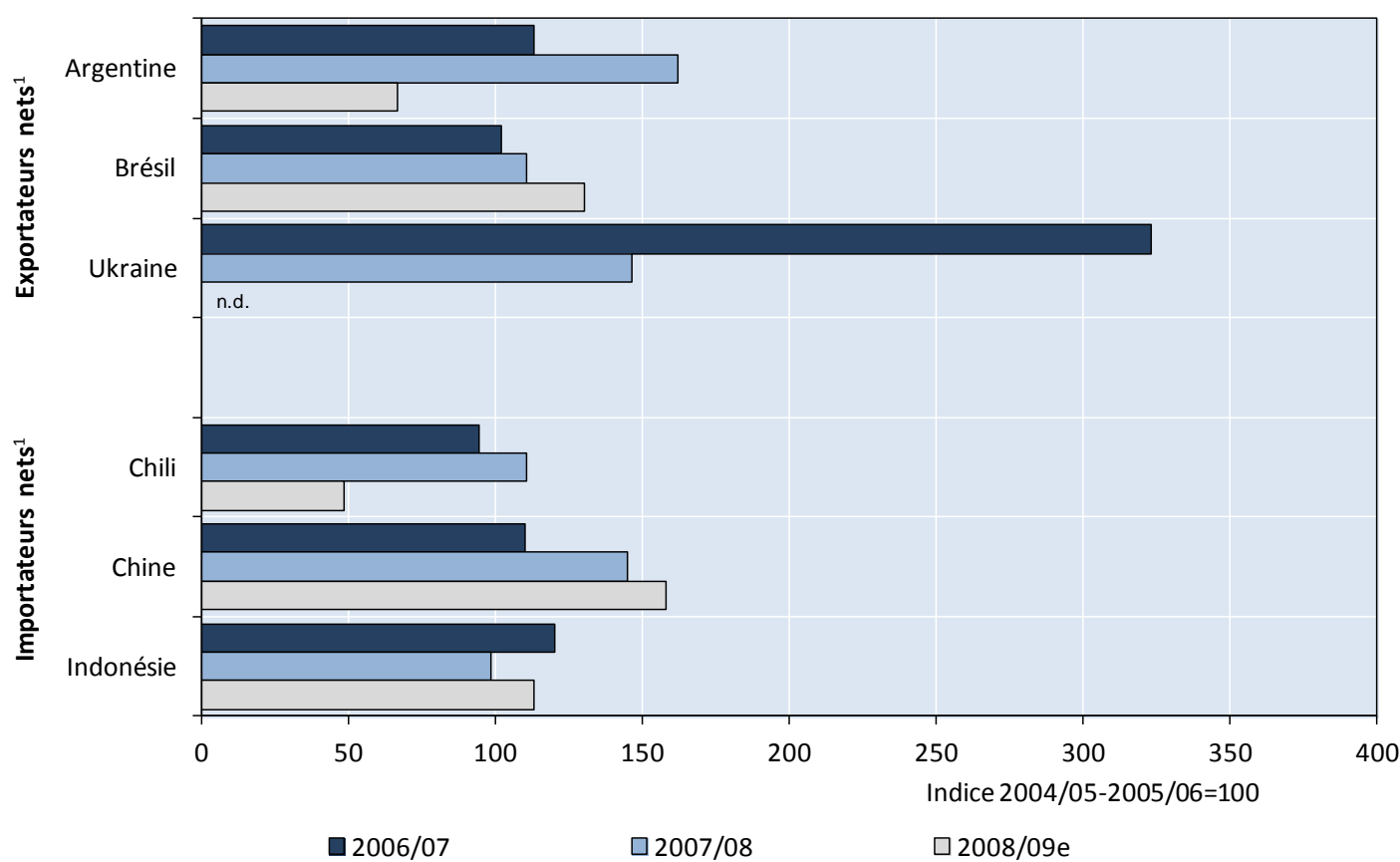

e : estimation ; n.d. : non disponible.

1. Pour déterminer si un pays doit être considéré comme exportateur ou importateur net de soja, on compare le volume moyen de ses exportations et de ses importations de ce produit au cours de la période biennale indiciaire 2004/05-2005/06. Le volume des échanges correspond aux exportations dans le cas des exportateurs nets et aux importations dans celui des importateurs nets.

Source : Conseil international des céréales (2010).

\section{Graphique 2.9. Exportations trimestrielles de soja de l'Argentine et de la Chine, 2006/07-2008/09}

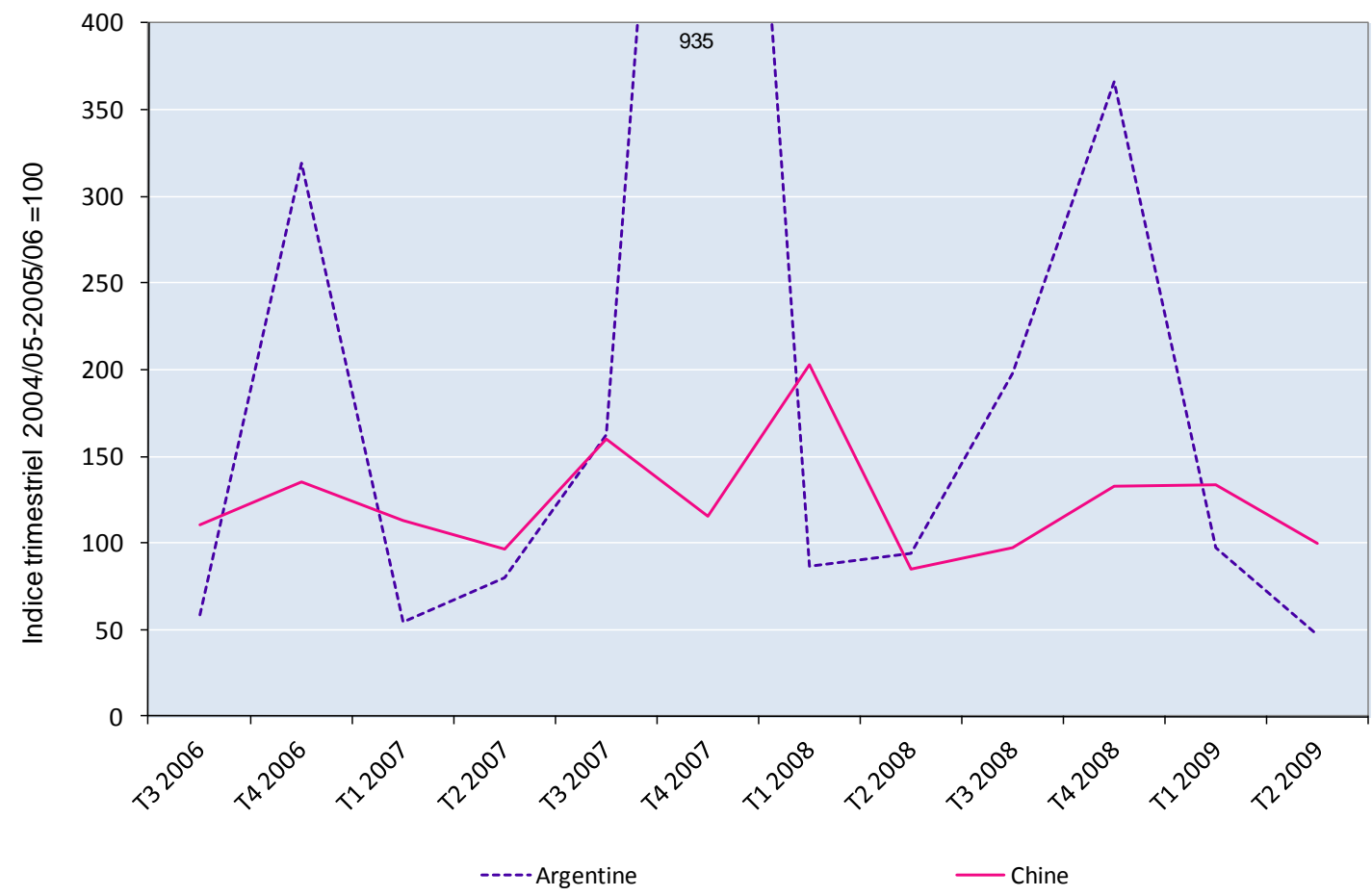

Source : Conseil international des céréales (2010). 


\subsection{Transmission internationale des prix ${ }^{5}$}

L'impact de la hausse des cours mondiaux sur les marchés intérieurs dépend entre autres choses du degré de transmission des prix mondiaux au sein des économies nationales. La transmission des prix pardelà les frontières dépend d'une multitude de variables. Compte tenu que la plupart des échanges de produits s'effectuent en dollars des États-Unis, les taux de change constituent l'un des principaux facteurs ayant une incidence sur la transmission des cours mondiaux exprimés en USD aux prix aux frontières exprimés dans la monnaie locale. L'appréciation des taux de change rend les importations meilleur marché, atténuant les répercussions de la hausse des cours, alors que la dépréciation des taux de change renchérit les importations. Sur le plan intérieur, la transmission des prix est influencée par des facteurs structurels tels que les coûts de transport, l'intensité de la concurrence et le degré de substitution entre les importations et la production intérieure, ainsi que par les mesures prises par les pouvoirs publics, telles que l'instauration d'obstacles aux échanges, de taxes sur les denrées alimentaires produites localement ou de subventions en leur faveur, ou encore les interventions visant à accroître les réserves alimentaires ou à puiser dans celles-ci. Les enseignements du passé portent à croire que la transmission des prix sera probablement très variable selon les produits, selon les pays et selon le moment considéré, et qu'elle est généralement inférieure à ce que l'on pourrait supposer a priori (Baffes et Gardner, 2003).

Le cœur de l'analyse consiste à calculer les variations des cours internationaux et des prix intérieurs des produits de base en termes réels (corrigés de l'inflation) entre le trimestre pertinent de 2006 et le même trimestre de 2008 et 2009 (tableau 2.2). Une moyenne trimestrielle est établie afin de lisser les fluctuations des prix d'un mois sur l'autre. Le trimestre pertinent est celui où les cours internationaux ont atteint leur niveau maximal en 2008 : le premier trimestre dans le cas du blé et le deuxième trimestre dans celui du maïs, du riz et du soja. Le même trimestre de l'année 2006 est utilisé afin de tenir compte des variations saisonnières. Les variations survenues entre 2006 et 2009 sont indiquées afin de montrer les évolutions qui se sont produites depuis la chute des cours internationaux des produits de base. À titre comparatif, le tableau 2.3 présente des calculs similaires pour la période 2003 à 2006 et pour 2003 à 2009. Cela aidera à interpréter certaines des évolutions observées durant cette période.

Entre 2006 et 2008 les cours mondiaux du blé, du maïs, du riz et du soja ont sensiblement augmenté et ont plus que doublé en dollars réels (colonne 1 des tableaux 2.2 et 2.3). ${ }^{6}$ Ils ont certes diminué entre 2008 et 2009 , mais leur baisse n'a toutefois pas été d'ampleur comparable, aussi demeurent-ils considérablement plus élevés en termes réels. Il convient d'observer qu'entre 2003 et 2006, les cours mondiaux du blé et du riz avaient également enregistré une hausse réelle de $7 \%$ et $40 \%$ respectivement. Par contre, les cours réels du maïs et du soja avaient baissé.

5. Cette section suit la procédure utilisée par Dawe (2008) pour examiner la transmission internationale des prix. Pour une étude plus complète et une analyse approfondie des questions de transmission des prix, voir Rapsomanikis et al., 2004.

6. Exprimés en termes réels en déflatant le prix international en USD par l'IPC des États-Unis pour tous les produits. Comme indiqué au tableau 2.1, les prix mondiaux représentatifs sont les suivants : pour le blé, le blé dur rouge d'hiver $\mathrm{n}^{\circ} 2$ des États-Unis, f.à.b. Golfe du Mexique; pour le maïs, maïs jaune $\mathrm{n}^{\circ} 2$ des États-Unis, f.à.b. Golfe du Mexique ; riz blanc thaïlandais de second choix 100\% B, f.à.b. Bangkok ; pour le soja, le soja jaune $n^{\circ} 1$ des États-Unis, f.à.b. Golfe du Mexique. 
La colonne 2 fait état de l'augmentation des cours mondiaux exprimée dans la monnaie locale de chacun des dix pays, sur les mêmes périodes de temps. ${ }^{7}$ Les hausses indiquées dans la colonne 2 sont plus faibles que dans la colonne 1 pour la plupart des pays, à l'exception de l'Afrique du Sud (dans la totalité des cas) et de l'Argentine (lorsque l'on compare le deuxième trimestre de 2006 et celui de 2009), pour lesquelles l'augmentation est plus marquée. Cette hausse plus modérée dans la colonne 2 tient au fait qu'au cours des périodes considérées bon nombre de pays ont bénéficié d'une appréciation en termes réels de leur monnaie par rapport au dollar des États-Unis, ce qui a neutralisé certains des effets de la hausse des prix exprimés en dollars (graphique 2.10). Par rapport aux huit autre monnaies, le rand sud-africain et le peso argentin ont en 2009 une bien moindre valeur réelle par rapport au dollar que ce n'était le cas en 2006, exacerbant par-là même les tensions suscitées par la hausse des cours mondiaux sur leurs marchés intérieurs.

7. Le taux de change réel entre deux pays est égal au produit du taux de change nominal et du niveau relatif des prix dans chacun des pays. Dans cette étude, le taux de change réel entre les États-Unis et le pays considéré au moment $t$ est défini comme suit : reri,j $=e i, j *(p t / p U S A, t)$ où $p$ correspond au niveau des prix dans le pays considéré, $p U S A$ au niveau des prix aux États-Unis, et $e i, j$ au taux de change nominal entre le dollar des États-Unis et la monnaie du pays considéré, exprimé en nombre de dollars par unité monétaire locale, de sorte que ei augmente en cas d'appréciation de la monnaie locale du pays considéré. 
Tableau 2.2. Transmission des cours mondiaux des produits de base sur les marchés intérieurs, 2006-08 et 2006-09

\begin{tabular}{|c|c|c|c|c|c|c|c|c|}
\hline \multirow{2}{*}{$\begin{array}{l}\text { Produit de } \\
\text { base et pays }\end{array}$} & \multicolumn{2}{|c|}{$\begin{array}{c}\text { (1) } \\
\text { Cours mondial } \\
\text { USD réels, \% de } \\
\text { variation }\end{array}$} & \multicolumn{2}{|c|}{$\begin{array}{c}\text { (2) } \\
\text { Cours mondial } \\
\begin{array}{c}\text { UML réelles, \% de } \\
\text { variation }\end{array}\end{array}$} & \multicolumn{2}{|c|}{$\begin{array}{c}\text { (3) } \\
\text { Prix intérieur } \\
\begin{array}{c}\text { UML réelles, \% de } \\
\text { variation }\end{array}\end{array}$} & \multicolumn{2}{|c|}{$\begin{array}{c}(4) \\
\text { Élasticité de la } \\
\text { transmission des prix. } \\
(3) /(2)\end{array}$} \\
\hline & $\begin{array}{c}\text { T1 } 2006- \\
\text { T1 } 2008 \\
\end{array}$ & $\begin{array}{c}\text { T1 } 2006- \\
\text { T1 } 2009 \\
\end{array}$ & $\begin{array}{l}\text { T1 } 2006- \\
\text { T1 } 2008\end{array}$ & $\begin{array}{c}\text { T1 } 2006- \\
\text { T1 } 2009 \\
\end{array}$ & $\begin{array}{l}\text { T1 } 2006- \\
\text { T1 } 2008 \\
\end{array}$ & $\begin{array}{c}\text { T1 } 2006- \\
\text { T1 } 2009 \\
\end{array}$ & $\begin{array}{c}\text { T1 } 2006- \\
\text { T1 } 2008 \\
\end{array}$ & $\begin{array}{c}\text { T1 } 2006- \\
\text { T1 } 2009 \\
\end{array}$ \\
\hline Argentine & 129 & 31 & 112 & 28 & 56 & 0 & 0.50 & 0.01 \\
\hline Brésil & 129 & 31 & 79 & 29 & 56 & 16 & 0.71 & 0.55 \\
\hline Chili & 129 & 31 & 93 & 39 & 62 & 15 & 0.66 & 0.38 \\
\hline Chine & 129 & 31 & 96 & 8 & 0 & 13 & 0.00 & 1.64 \\
\hline Inde & 129 & 31 & 93 & 26 & -2 & -5 & -0.02 & -0.20 \\
\hline Indonésie & 129 & 31 & 114 & 42 & 74 & 53 & 0.64 & 1.24 \\
\hline Russie & 129 & 31 & 73 & 22 & 82 & -11 & 1.12 & -0.50 \\
\hline Afrique du Sud & 129 & 31 & 165 & 86 & 132 & 58 & 0.80 & 0.68 \\
\hline Ukraine & 129 & 31 & 81 & 31 & 50 & 7 & 0.62 & 0.21 \\
\hline Vietnam & 129 & 31 & 98 & 5 & n.d. & n.d. & n.d. & n.d. \\
\hline Maïs & $\begin{array}{l}\text { T2 } 2006- \\
\text { T2 } 2008\end{array}$ & $\begin{array}{l}\text { T2 } 2006- \\
\text { T2 } 2009\end{array}$ & $\begin{array}{l}\text { T2 } 2006- \\
\text { T2 } 2008\end{array}$ & $\begin{array}{l}\text { T2 } 2006- \\
\text { T2 } 2009\end{array}$ & $\begin{array}{l}\text { T2 } 2006- \\
\text { T2 } 2008\end{array}$ & $\begin{array}{l}\text { T2 } 2006- \\
\text { T2 } 2009\end{array}$ & $\begin{array}{l}\text { T2 } 2006- \\
\text { T2 } 2008\end{array}$ & $\begin{array}{l}\text { T2 } 2006- \\
\text { T2 } 2009\end{array}$ \\
\hline Argentine & 120 & 53 & 102 & 57 & 66 & 19 & 0.65 & 0.33 \\
\hline Brésil & 120 & 53 & 63 & 34 & 67 & 25 & 1.05 & 0.74 \\
\hline Chili & 120 & 53 & 88 & 51 & 68 & 40 & 0.77 & 0.78 \\
\hline Chine & 120 & 53 & 83 & 25 & 14 & 10 & 0.17 & 0.39 \\
\hline Inde & 120 & 53 & 88 & 39 & 3 & 8 & 0.04 & 0.19 \\
\hline Indonésie & 120 & 53 & 105 & 52 & 12 & 20 & 0.12 & 0.38 \\
\hline Russie & 120 & 53 & 65 & 37 & 116 & 8 & 1.78 & 0.22 \\
\hline Afrique du Sud & 120 & 53 & 143 & 69 & 37 & 7 & 0.26 & 0.10 \\
\hline Ukraine & 120 & 53 & 59 & 47 & 48 & 9 & 0.81 & 0.19 \\
\hline Vietnam & 120 & 53 & 78 & 21 & n.d. & n.d. & n.d. & n.d. \\
\hline Riz & $\begin{array}{l}\text { T2 } 2006- \\
\text { T2 } 2008\end{array}$ & $\begin{array}{l}\text { T2 } 2006- \\
\text { T2 } 2009\end{array}$ & $\begin{array}{l}\text { T2 } 2006- \\
\text { T2 } 2008\end{array}$ & $\begin{array}{l}\text { T2 } 2006- \\
\text { T2 } 2009\end{array}$ & $\begin{array}{l}\text { T2 } 2006- \\
\text { T2 } 2008\end{array}$ & $\begin{array}{l}\text { T2 } 2006- \\
\text { T2 } 2009\end{array}$ & $\begin{array}{l}\text { T2 } 2006- \\
\text { T2 } 2008 \\
\end{array}$ & $\begin{array}{l}\text { T2 } 2006- \\
\text { T2 } 2009 \\
\end{array}$ \\
\hline Argentine & 166 & 74 & 144 & 79 & n.d. & n.d. & n.d. & n.d. \\
\hline Brésil & 166 & 74 & 98 & 53 & 52 & 18 & 0.53 & 0.34 \\
\hline Chili & 166 & 74 & 127 & 72 & 51 & 38 & 0.40 & 0.53 \\
\hline Chine & 166 & 74 & 122 & 43 & -6 & 4 & -0.05 & 0.10 \\
\hline Inde & 166 & 74 & 128 & 59 & 0 & 6 & 0.00 & 0.11 \\
\hline Indonésie & 166 & 74 & 148 & 74 & 9 & 8 & 0.06 & 0.11 \\
\hline Russie & 166 & 74 & 100 & 57 & n.d. & n.d. & n.d. & n.d. \\
\hline Afrique du Sud & 166 & 74 & 194 & 93 & n.d. & n.d. & n.d. & n.d. \\
\hline Ukraine & 166 & 74 & 93 & 68 & n.d. & n.d. & n.d. & n.d. \\
\hline Vietnam & 166 & 74 & 115 & 38 & 155 & 27 & 1.34 & 0.71 \\
\hline Soja & $\begin{array}{l}\text { T2 } 2006- \\
\text { T2 } 2008\end{array}$ & $\begin{array}{l}\text { T2 } 2006- \\
\text { T2 } 2009\end{array}$ & $\begin{array}{l}\text { T2 } 2006- \\
\text { T2 } 2008\end{array}$ & $\begin{array}{l}\text { T2 } 2006- \\
\text { T2 } 2009\end{array}$ & $\begin{array}{l}\text { T2 } 2006- \\
\text { T2 } 2008\end{array}$ & $\begin{array}{l}\text { T2 } 2006- \\
\text { T2 } 2009\end{array}$ & $\begin{array}{l}\text { T2 } 2006- \\
\text { T2 } 2008\end{array}$ & $\begin{array}{l}\text { T2 } 2006- \\
\text { T2 } 2009 \\
\end{array}$ \\
\hline Argentine & 116 & 80 & 98 & 85 & 45 & 54 & 0.45 & 0.64 \\
\hline Brésil & 116 & 80 & 61 & 57 & 69 & 69 & 1.14 & 1.20 \\
\hline Chili & 116 & 80 & 85 & 77 & n.d. & n.d. & n.d. & n.d. \\
\hline Chine & 116 & 80 & 80 & 48 & 74 & 27 & 0.93 & 0.56 \\
\hline Inde & 116 & 80 & 86 & 63 & 44 & 59 & 0.51 & 0.93 \\
\hline Indonésie & 116 & 80 & 102 & 79 & 6 & 25 & 0.06 & 0.31 \\
\hline Russie & 116 & 80 & 62 & 61 & n.d. & n.d. & n.d. & n.d. \\
\hline Afrique du Sud & 116 & 80 & 140 & 98 & 135 & 74 & 0.97 & 0.76 \\
\hline Ukraine & 116 & 80 & 57 & 73 & 84 & 67 & 1.48 & 0.92 \\
\hline Vietnam & 116 & 80 & 75 & 42 & n.d. & n.d. & n.d. & n.d. \\
\hline
\end{tabular}

n.d. : non disponible.

Source: Calculs de l'auteur à partir des données issues des statistiques financières internationales du FMI (2010), de la base de données et outil d'analyse du prix national des aliments développé par le SMIAR et la FAO (2010), des rapports du Réseau d'information sur l'agriculture mondiale (Global Agriculture Information Network - GAIN) du Service de l'agriculture extérieure de I'USDA, ainsi que des organismes statistiques nationaux. 
Tableau 2.3. Transmission des cours mondiaux des produits de base sur les marchés intérieurs, 2003-06 et 2003-09

\begin{tabular}{|c|c|c|c|c|c|c|c|c|}
\hline \multirow{2}{*}{ 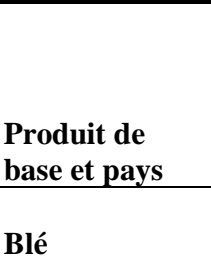 } & \multicolumn{2}{|c|}{$\begin{array}{c}\text { (1) } \\
\text { Cours mondial } \\
\begin{array}{c}\text { USD réels, \% de } \\
\text { variation }\end{array} \\
\end{array}$} & \multicolumn{2}{|c|}{$\begin{array}{c}(2) \\
\text { Cours mondial } \\
\text { UML réelles, \% de } \\
\text { variation }\end{array}$} & \multicolumn{2}{|c|}{$\begin{array}{c}\text { (3) } \\
\text { Prix intérieur } \\
\begin{array}{c}\text { UML réelles, \% de } \\
\text { variation }\end{array}\end{array}$} & \multicolumn{2}{|c|}{$\begin{array}{c}(4) \\
\text { Élasticité de la } \\
\text { transmission des prix. } \\
(3) /(2)\end{array}$} \\
\hline & $\begin{array}{l}\text { T1 } 2003- \\
\text { T1 } 2006\end{array}$ & $\begin{array}{c}\text { T1 } 2003- \\
\text { T1 } 2009 \\
\end{array}$ & $\begin{array}{l}\text { T1 } 2003- \\
\text { T1 } 2006\end{array}$ & $\begin{array}{c}\text { T1 } 2003- \\
\text { T1 } 2009\end{array}$ & $\begin{array}{l}\text { T1 } 2003- \\
\text { T1 } 2006\end{array}$ & $\begin{array}{c}\text { T1 } 2003- \\
\text { T1 } 2009\end{array}$ & $\begin{array}{l}\text { T1 } 2003- \\
\text { T1 } 2006\end{array}$ & $\begin{array}{c}\text { T1 } 2003- \\
\text { T1 } 2009\end{array}$ \\
\hline Argentine & 7 & 41 & -8 & 18 & -26 & -26 & 3.22 & -1.46 \\
\hline Brésil & 7 & 41 & -40 & -22 & -46 & -38 & 1.17 & 1.72 \\
\hline Chili & 7 & 41 & -22 & 9 & -19 & -7 & 0.86 & -0.79 \\
\hline Chine & 7 & 41 & 6 & 14 & 22 & 38 & 3.82 & 2.71 \\
\hline Inde & 7 & 41 & -4 & 20 & 2 & -4 & -0.40 & -0.18 \\
\hline Indonésie & 7 & 41 & -8 & 31 & -25 & 14 & 3.05 & 0.46 \\
\hline Russie & 7 & 41 & -25 & -9 & n.d. & n.d. & n.d. & n.d. \\
\hline Afrique du Sud & 7 & 41 & -16 & 56 & -6 & 49 & 0.38 & 0.87 \\
\hline Ukraine & 7 & 41 & -18 & 8 & -21 & -15 & 1.17 & -1.85 \\
\hline Vietnam & 7 & 41 & -2 & 3 & n.d. & n.d. & n.d. & n.d. \\
\hline Maïs & $\begin{array}{l}\text { T2 } 2003- \\
\text { T2 } 2006\end{array}$ & $\begin{array}{l}\text { T2 } 2003- \\
\text { T2 } 2009\end{array}$ & $\begin{array}{l}\text { T2 } 2003- \\
\text { T2 } 2006\end{array}$ & $\begin{array}{l}\text { T2 } 2003- \\
\text { T2 } 2009\end{array}$ & $\begin{array}{l}\text { T2 } 2003- \\
\text { T2 } 2006\end{array}$ & $\begin{array}{l}\text { T2 } 2003- \\
\text { T2 } 2009\end{array}$ & $\begin{array}{l}\text { T2 } 2003- \\
\text { T2 } 2006\end{array}$ & $\begin{array}{l}\text { T2 } 2003- \\
\text { T2 } 2009\end{array}$ \\
\hline Argentine & -7 & 42 & -12 & 39 & -8 & 9 & 0.73 & 0.23 \\
\hline Brésil & -7 & 42 & -37 & -15 & -38 & -22 & 1.02 & 1.42 \\
\hline Chili & -7 & 42 & -29 & 7 & -21 & 10 & 0.73 & 1.50 \\
\hline Chine & -7 & 42 & -8 & 15 & 20 & 32 & -2.48 & 2.09 \\
\hline Inde & -7 & 42 & -13 & 21 & -5 & 3 & 0.36 & 0.12 \\
\hline Indonésie & -7 & 42 & -17 & 26 & -7 & 11 & 0.42 & 0.43 \\
\hline Russie & -7 & 42 & -34 & -10 & n.d. & n.d. & n.d. & n.d. \\
\hline Afrique du Sud & -7 & 42 & -17 & 40 & 35 & 44 & -2.06 & 1.10 \\
\hline Ukraine & -7 & 42 & -27 & 8 & -46 & -41 & 1.71 & -5.17 \\
\hline Vietnam & -7 & 42 & -15 & 3 & n.d. & n.d. & n.d. & n.d. \\
\hline Riz & $\begin{array}{l}\text { T2 } 2003- \\
\text { T2 } 2006\end{array}$ & $\begin{array}{l}\text { T2 } 2003- \\
\text { T2 } 2009\end{array}$ & $\begin{array}{l}\text { T2 } 2003- \\
\text { T2 } 2006\end{array}$ & $\begin{array}{l}\text { T2 } 2003- \\
\text { T2 } 2009\end{array}$ & $\begin{array}{l}\text { T2 } 2003- \\
\text { T2 } 2006\end{array}$ & $\begin{array}{l}\text { T2 } 2003- \\
\text { T2 } 2009\end{array}$ & $\begin{array}{l}\text { T2 } 2003- \\
\text { T2 } 2006\end{array}$ & $\begin{array}{l}\text { T2 } 2003- \\
\text { T2 } 2009\end{array}$ \\
\hline Argentine & 40 & 145 & 34 & 140 & n.d. & n.d. & n.d. & n.d. \\
\hline Brésil & 40 & 145 & -4 & 46 & -39 & -28 & 8.73 & -0.60 \\
\hline Chili & 40 & 145 & 7 & 84 & -18 & 13 & -2.52 & 0.16 \\
\hline Chine & 40 & 145 & 39 & 99 & 42 & 49 & 1.08 & 0.49 \\
\hline Inde & 40 & 145 & 32 & 110 & -8 & -2 & -0.26 & -0.02 \\
\hline Indonésie & 40 & 145 & 25 & 118 & 27 & 37 & 1.05 & 0.31 \\
\hline Russie & 40 & 145 & -1 & 56 & n.d. & n.d. & n.d. & n.d. \\
\hline Afrique du Sud & 40 & 145 & 26 & 142 & n.d. & n.d. & n.d. & n.d. \\
\hline Ukraine & 40 & 145 & 11 & 86 & n.d. & n.d. & n.d. & n.d. \\
\hline Vietnam & 40 & 145 & 28 & 77 & 16 & 47 & 0.56 & 0.61 \\
\hline Soja & $\begin{array}{l}\text { T2 } 2003- \\
\text { T2 } 2006\end{array}$ & $\begin{array}{l}\text { T2 } 2003- \\
\text { T2 } 2006\end{array}$ & $\begin{array}{l}\text { T2 } 2003- \\
\text { T2 } 2006\end{array}$ & $\begin{array}{l}\text { T2 } 2003- \\
\text { T2 } 2009\end{array}$ & $\begin{array}{l}\text { T2 } 2003- \\
\text { T2 } 2006\end{array}$ & $\begin{array}{l}\text { T2 } 2003- \\
\text { T2 } 2009\end{array}$ & $\begin{array}{l}\text { T2 } 2003- \\
\text { T2 } 2006\end{array}$ & $\begin{array}{l}\text { T2 } 2003- \\
\text { T2 } 2009\end{array}$ \\
\hline Argentine & -13 & 57 & -17 & 54 & -13 & 34 & 0.78 & 0.63 \\
\hline Brésil & -13 & 57 & -41 & -7 & -43 & -3 & 1.05 & 0.47 \\
\hline Chili & -13 & 57 & -33 & 18 & n.d. & n.d. & n.d. & n.d. \\
\hline Chine & -13 & 57 & -14 & 28 & -13 & 10 & 0.95 & 0.38 \\
\hline Inde & -13 & 57 & -18 & 34 & -25 & 19 & 1.40 & 0.56 \\
\hline Indonésie & -13 & 57 & -22 & 39 & 19 & 48 & -0.84 & 1.22 \\
\hline Russie & -13 & 57 & -38 & 0 & n.d. & n.d. & n.d. & n.d. \\
\hline Afrique du Sud & -13 & 57 & -22 & 55 & -19 & 41 & 0.87 & 0.75 \\
\hline Ukraine & -13 & 57 & -31 & 19 & -28 & 20 & 0.91 & 1.04 \\
\hline Vietnam & -13 & 57 & -20 & 14 & n.d. & n.d. & n.d. & n.d. \\
\hline
\end{tabular}

n.d. : non disponible.

Source : Calculs de l'auteur à partir des données issues des statistiques financières internationales du FMI (2010), de la base de données et outil d'analyse du prix national des aliments développé par le SMIAR et la FAO (2010), des rapports du Réseau d'information sur l'agriculture mondiale (Global Agriculture Information Network - GAIN) du Service de l'agriculture extérieure de I'USDA, ainsi que des organismes statistiques nationaux. 


\section{Graphique 2.10. Variation des taux de change réels UML-USD entre 2006-08 et 2006-09}

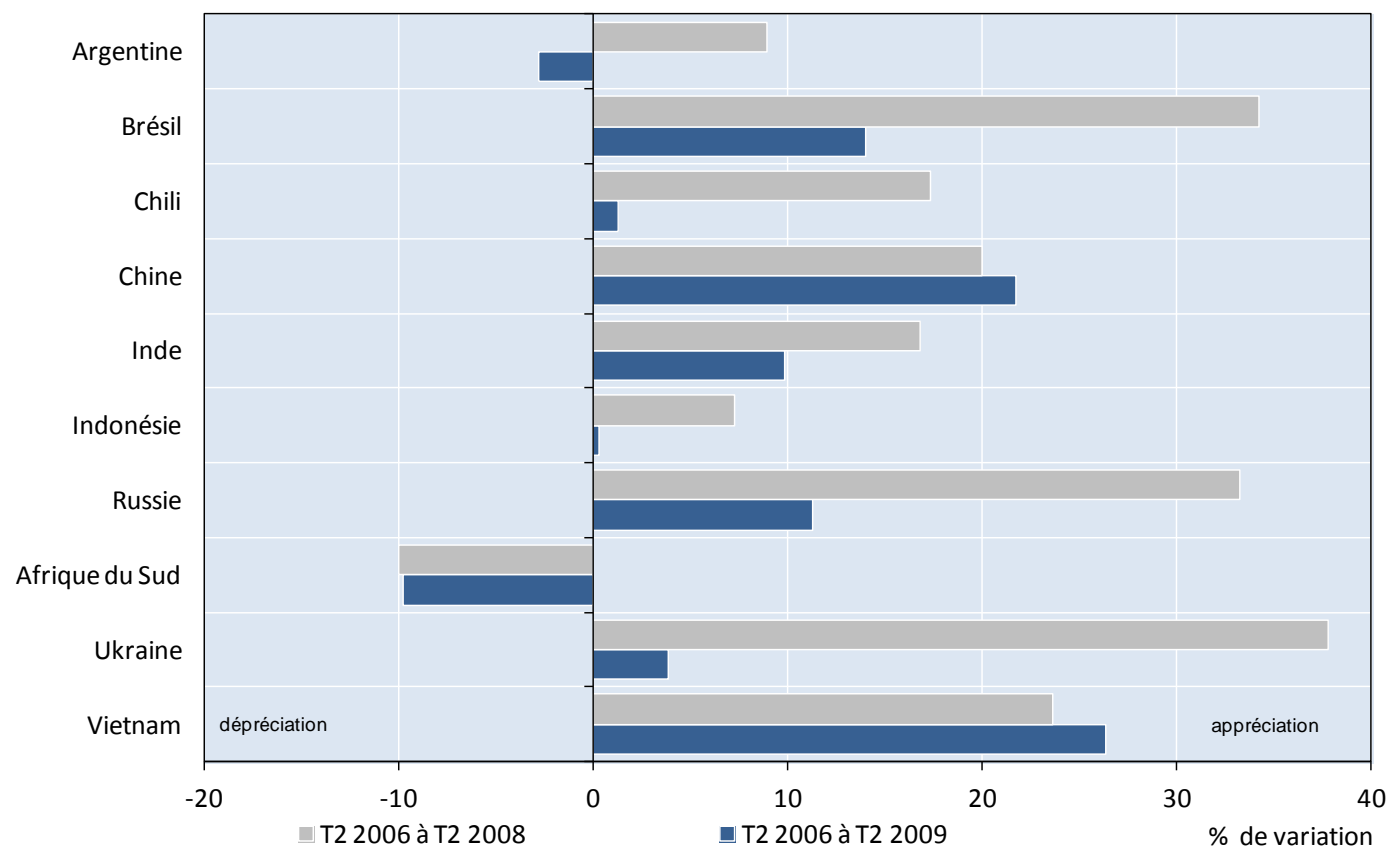

Source : FMI, Base de données des statistiques financières internationales (2010).

L'appréciation générale des monnaies par au dollar des États-Unis a été particulièrement marquée entre le premier semestre 2006 et le premier semestre 2008. L'évolution des conditions macroéconomiques et de la politique monétaire étaient à l'origine de cette tendance générale. En août 2007, la Réserve fédérale des États-Unis a commencé à baisser les taux d'intérêt, dans un effort pour stimuler une économie américaine en perte de vitesse. Cette initiative a encouragé les investisseurs à se tourner vers d'autres monnaies offrant des taux d'intérêt plus élevés, entraînant une dépréciation du dollar face à la plupart des autres monnaies. En juillet 2008, lorsqu'il est apparu que le reste du monde allait tout droit vers une récession, beaucoup de monnaies ont commencé à se déprécier par rapport au dollar à mesure que les investisseurs se tournaient de nouveau vers les titres de la dette publique des États-Unis. Preuve en est la baisse du taux d'appréciation au cours de la période triennale 2006-09, comparativement à 2006-08. En termes absolus, c'est au Brésil, au Chili, en Russie et en Ukraine que la perte de valeur de la monnaie a été la plus forte. La Chine et le Vietnam font exception, puisque le taux de change réel des monnaies de ces deux pays a continué de s'apprécier par rapport au dollar des États-Unis, tandis que le rand sud-africain est resté relativement stable en termes réels.

La colonne 3 des tableaux 2.2 et 2.3 montrent les variations des prix intérieurs réels au niveau de la vente en gros. Pour la plupart des pays et des produits de base, les variations, les variations des prix intérieurs sont inférieures à celles des cours internationaux lorsqu'elles sont mesurées dans la monnaie locale. L'élasticité de la transmission des prix est calculée en divisant le pourcentage de variation du prix intérieur par celui du cours international mesuré en unités monétaires locales. Cela fait apparaître dans la plupart des cas une élasticité de la transmission inférieures à un (colonne 4). De manière générale, le degré de transmission des variations des cours mondiaux aux prix intérieurs est d'autant plus élevé que cette valeur est proche de l'unité. Cependant, une élasticité égale à un n'indique pas pour autant une transmission parfaite ou intégrale des prix (Sharma, 2003). Si le prix intérieur de départ est inférieur au prix aux frontières initial, comme cela peut se produire dans le cas d'un pays exportateur, la même variation des prix en termes absolus se traduira par une élasticité supérieure à un. ${ }^{8} \mathrm{Si}$ par contre le prix

8. De même, s'il existe une différence de prix initiale, en raison par exemple de différences de qualité, une variation de $50 \%$ des deux prix se traduit par un taux de transmission des prix égal à un, mais la différence entre les deux prix sera plus forte qu'au départ en termes absolus. 
intérieur de départ est supérieur au prix aux frontières initial, par exemple dans le cas d'un pays importateur ayant établi une protection tarifaire, la même variation des prix en valeur absolue se traduira également par une élasticité supérieure à un. En outre, l'élasticité observée sera d'autant plus éloignée de l'unité que les coûts de transport et de commercialisation et les droits de douane à l'importation ou à l'exportation sont élevés.

Graphique 2.11. Transmission des cours mondiaux sur le marché intérieur, 2006-08
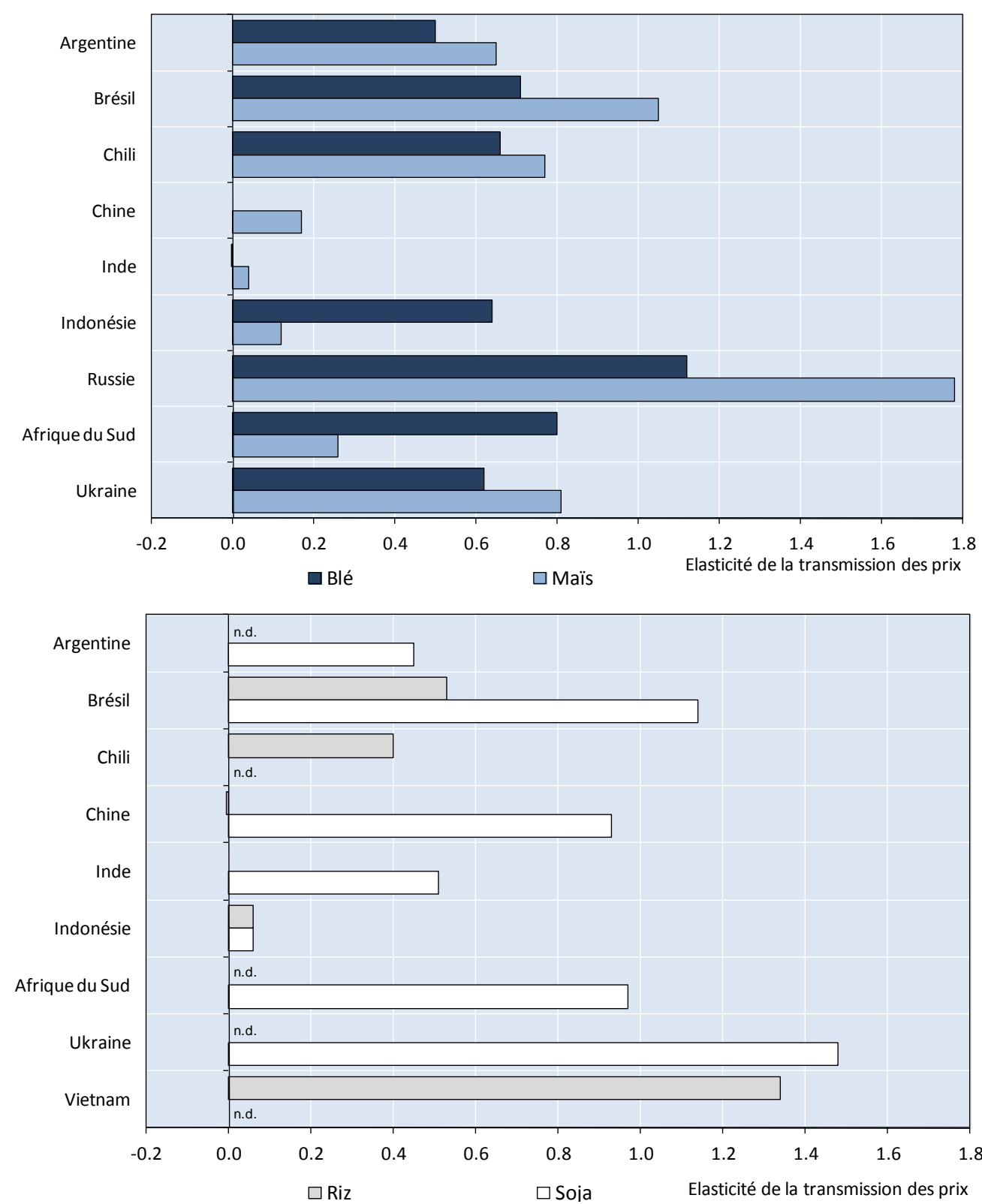

n.d. : non disponible.

Source: Calculs de l'auteur à partir des données issues des statistiques financières internationales du FMI (2010), de la base de données et outil d'analyse du prix national des aliments développé par le SMIAR et la FAO (2010), des rapports du Réseau d'information sur l'agriculture mondiale (Global Agriculture Information Network - GAIN) du Service de l'agriculture extérieure de I'USDA, ainsi que des organismes statistiques nationaux.

Le graphique 2.8 présente l'élasticité de la transmission des prix de 2006 à 2008 pour les quatre produits de base. Les comparaisons entre les pays font apparaître que l'élasticité de la transmission des prix 
pour les trois céréales en Chine et en Inde, et pour le riz et le soja en Indonésie est sensiblement plus faible que pour les autres pays. De 2006 à 2008, le prix réel du blé est demeuré constant en Chine et a baissé de $2 \%$ en Inde, alors que le prix réel du riz en Inde est resté stable en Inde et a baissé de $6 \%$ en Chine, bien que les cours mondiaux du blé et du riz exprimés en monnaie locale aient plus que doublé en termes réels. Les mesures prises par ces trois pays, conjuguées à une série de bonnes récoltes, ont bien plus efficacement isolé le marché intérieur des variations observées sur les marchés internationaux. Le niveau plus élevé de transmission des prix enregistré en Chine et en Inde pour le soja au cours de la même période confirme cette constatation. L'Indonésie a pris plusieurs initiatives pour réduire l'incidence de la hausse des cours du soja sur les prix en vigueur sur son marché intérieur (suppression des droits de douane et de la TVA, et subventions aux transformateurs), et elles paraissent avoir donné de bons résultats. La comparaison de la situation actuelle et de celle de 2003-06 met également en évidence l'impact de certaines évolutions de l'action des pouvoirs publics (tableau 2.3). Au cours de cette période plus ancienne, les prix du riz en Chine et en Indonésie ont augmenté au même rythme que les cours mondiaux, l'élasticité de la transmission étant respectivement de 1.08 et 1.05. En Inde, le prix réel du riz a chuté de 8\% en 2003-06 alors que les cours mondiaux du riz exprimés dans la monnaie locale ont augmenté de $32 \%$ en termes réels.

Contrairement à celles mises en œuvre dans les trois autres pays d'Asie, les mesures prises au Vietnam pour contrôler les prix du riz n'ont pas isolé le marché intérieur des évolutions des cours mondiaux. La variation des cours mondiaux en termes absolus s'est intégralement répercutée sur le marché intérieur durant la période 2006-08. C'est pour partie une conséquence des interventions des spéculateurs locaux sur le marché intérieur du riz, qui ont entraîné un doublement des prix à Ho Chi Minh Ville en l'espace d'un week-end à la fin avril 2008. Lorsque la détente des prix est survenue, la demande internationale de riz s'était pour une large part évanouie, laissant de vastes stocks invendus de riz de première qualité acheté au prix fort que les organismes exportateurs provinciaux ne parvenaient pas à écouler. Confrontés à des taux d'intérêt de $19 \%$ et à des prix minimums à l'exportation extrêmement élevés, les organismes provinciaux exportateurs de denrées alimentaires se sont pour une large part abstenus de procéder à de nouveaux achats de riz d'été ou d'automne à forte teneur en eau, contribuant à un effondrement des prix intérieurs en août-novembre 2008 qui s'est soldé par un important manque-àgagner pour les agriculteurs, ainsi que par la perte de recettes d'exportation (Slayton, 2009).

Bien que l'Ukraine ait limité avec succès le volume de ses exportations de céréales, l'effet de cette mesure du point de vue de la réduction du degré de transmission de la hausse des cours internationaux sur le marché international y a été plus faible en termes relatifs qu'en Chine ou en Inde au cours de la période 2006-08. Cela est dû au fait que l'augmentation globale de l'offre intérieure de céréales était trop faible pour entraîner une réduction spectaculaire des prix (UkrAgroConsult, 2009). Une des raisons en est que les exportateurs de céréales se sont tournés vers la production de farine (qui ne faisait pas l'objet de contingents d'exportation) afin de contourner les contingents d'exportation applicables aux céréales. Aussi les exportations de farine de blé ont-elles atteint un niveau sans précédent en 2007/08. Un autre facteur tenait à la ferme volonté de nombreux producteurs et négociants de stocker les céréales jusqu'à ce que les prix atteignent les niveaux souhaités. Le bon prix tiré des ventes de colza, de soja et de tournesol permettaient à bon nombre de producteurs de conserver des stocks. Les niveaux élevés des stocks se sont conjugués à la récolte exceptionnelle qui a suivi en 2008 pour aboutir à une large baisse de prix de toutes les grandes céréales en 2008/09, les sociétés de commerce extérieur ralentissant leurs achats par crainte que les prix continuent de chuter.

Contrairement à l'Ukraine, l'Argentine a un peu mieux réussi à isoler son marché intérieur de la hausse des prix. Bien que les statistiques commerciales portent à croire que les mesures visant à imposer des restrictions aux exportations de céréales n'ont pas sensiblement réduit les volumes exportés par rapport à la période antérieure, il n'en est pas moins plus que probable qu'elles les aient maintenus à un niveau bien inférieur à celui qu'ils n'auraient pas manqué d'atteindre en leur absence, compte tenu de la hausse des cours mondiaux et des bas prix en vigueur sur le marché intérieur. Qui plus est, l'augmentation des 
taxes à l'exportation a également permis de conserver un faible degré de transmission des cours internationaux sur le marché intérieur.

De manière générale, les taux de transmission des prix sont bien plus élevés en Afrique du Sud, au Brésil, au Chile et en Russie. Le faible degré de transmission des prix du maïs observé en Afrique du Sud au cours de la période 2006-08 est une conséquence de la mauvaise récolte de maïs de 2006. Par la suite, lorsque les cours mondiaux ont commencé à augmenter à partir de 2006, le prix intérieur était déjà élevé et s'est contenté de se maintenir au même point. C'est ce que corrobore le tableau 2.3, qui indique un taux de transmission de -2.06 pour la période 2003-06 du fait de l'augmentation des prix intérieurs alors même que les cours mondiaux s'orientaient à la baisse, contre un taux de transmission de 1.10 pour la période 2003-09. Au Chili, il s'est produit une baisse sensible du prix du blé sur le marché intérieur au cours de la période 2008-09. Dans le même temps, les prix de la farine et du blé sont demeurés relativement constants. Cela a suscité des protestations de la part de centaines d'exploitants exigeant une enquête publique sur la filière «blé-farine-pain». À la suite de cela, le «Bureau national des procureurs chargés des affaires économiques » (Fiscalía Nacional Económica-FNE) a été chargé d'enquêter sur les irrégularités en matière de prix.

\subsection{Prix à la consommation des produits alimentaires et inflation}

Si les sections précédentes se sont axées sur le degré de transmission des cours internationaux des produits de base sur les marchés de gros nationaux, celle-ci analyse dans quelle mesure les prix des denrées alimentaires ont effectivement connu une hausse. Afin de les mettre en perspective, les variations des prix des denrées alimentaires observées dans les dix pays examinés sont comparées à leur moyenne pondérée dans l'ensemble de la zone de l'OCDE. ${ }^{9}$ L'analyse porte sur des périodes annuelles finissant en juin et non sur les années civiles, afin de maintenir la cohérence avec l'examen de la transmission des prix, qui tient quant à lui compte des points d'inflexion des cours internationaux des céréales et des oléagineux. Un tournant majeur a par ailleurs pu être observé dans la politique des autorités monétaires au cours de la seconde moitié de 2008, dont l'orientation relativement restrictive destinée à limiter les effets inflationnistes de la hausse des prix des denrées alimentaires et de ceux de l'énergie a cédé la place à une orientation expansionniste visant à combattre les effets de la crise du crédit et du ralentissement économique mondial.

Les dix pays ont tous subi une augmentation des prix à la consommation annuels moyens des denrées alimentaires entre les périodes 2003/04-05/06 et 2006/07-07/08 (graphique 2.12). L'Afrique du Sud et le Chili, où les hausses des prix des denrées alimentaires avaient été les plus faibles au cours de la période la plus ancienne, ont connu la plus forte augmentation des prix alimentaires. Le taux d'augmentation annuel moyen des denrées alimentaires est passé en Afrique du Sud de 2.5\% en 2003/04-05/06 à 10.5\% en 2006/07-07/08 et au Chili de 1.2\% à 9.8\% respectivement. L'Afrique du Sud et le Chili sont deux petites économies relativement ouvertes, caractérisées par une bonne transmission des prix et qui n'ont rien fait pour altérer par leurs interventions les signaux de prix du marché. Le taux d'augmentation des prix à la consommation des denrées alimentaires a plus que doublé d'une période à l'autre en Chine et en Inde, malgré le succès avec lequel ces pays ont réduit la transmission des prix des céréales.

Le taux d'augmentation des prix à la consommation des denrées alimentaires a quasiment doublé dans la zone de 1'OCDE, passant de 2.1\% en 2003/04-05/06 à 3.9\% en 2006/07-07/08, mais est demeuré plus bas que dans toutes les économies émergentes sur lesquelles porte l'étude. La plus faible hausse des prix alimentaires dans la zone de l'OCDE reflète tout à la fois la plus forte proportion d'aliments transformés

9. Comme pour les totaux calculés par l'OCDE pour les six autres régions du monde, il s'agit d'un indice de Laspeyres en chaîne annuelle ou les coefficients de pondération par pays de chacun des maillons sont fonction des dépenses de consommation finale privée des ménages au cours de l'année précédente. 
consommés dans ses pays membres, comparativement aux économies émergentes ou en développement et la diminution de la part des prix des produits de base dans les coûts de transformation totaux au profit d'autres éléments tels que des salaires plus élevés. Par conséquent, une augmentation du prix des produits de base contribue relativement moins à la hausse des prix à la consommation des denrées alimentaires dans les pays de l'OCDE.

\section{Graphique 2.12. Taux de variation annuel moyen des prix à la consommation des denrées alimentaires - 2003/04-05/06 et 2006/07-07/08}

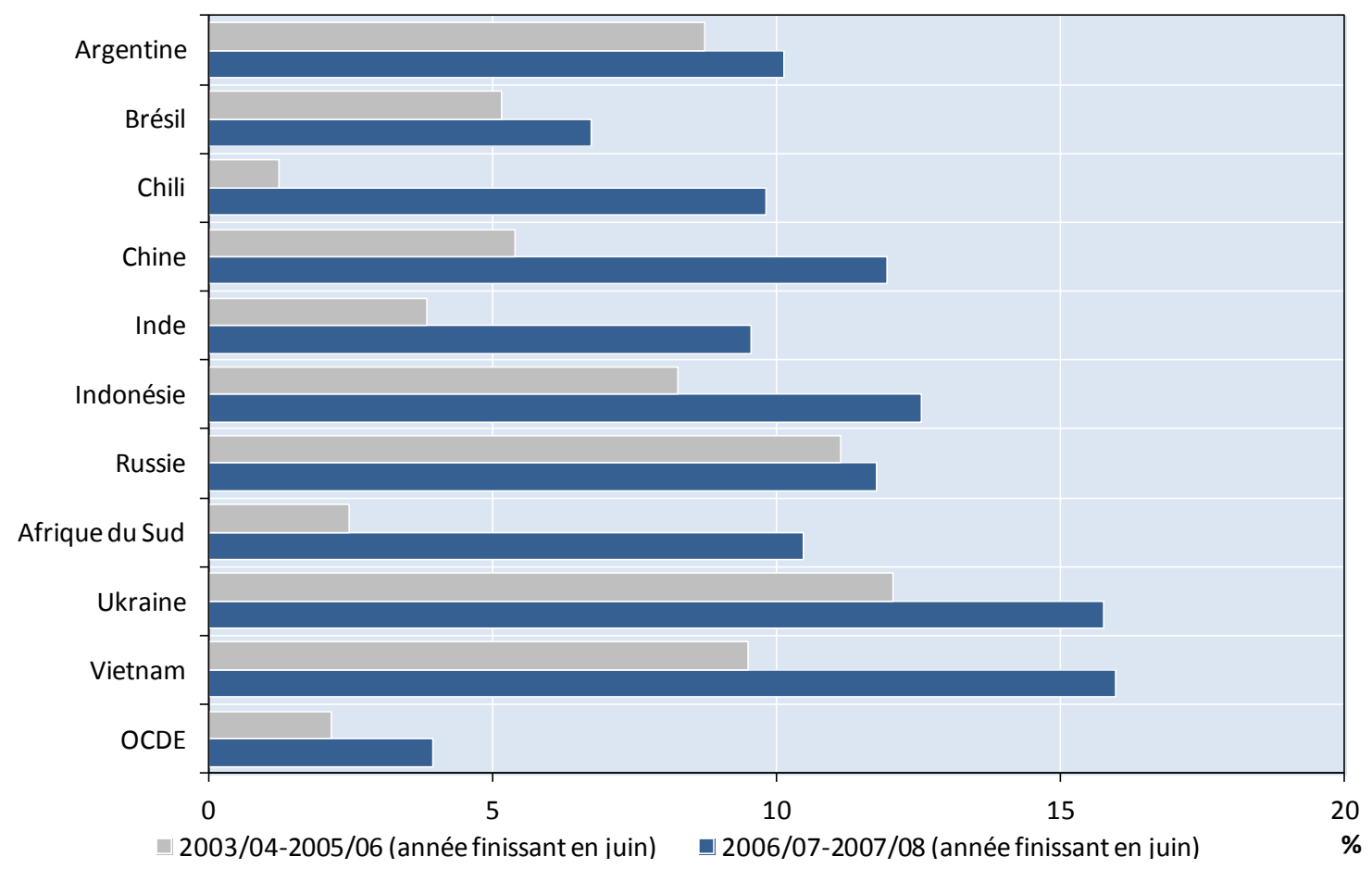

Source : Base statistique de l'OCDE et organismes statistiques nationaux.

Pour la moitié des dix pays, l'augmentation des prix à la consommation des denrées alimentaires a atteint son niveau maximal dans l'année finissant en juin 2008, leur renchérissement se produisant à un rythme plus lent en 2008/09 (tableau 2.4). Des baisses marquées du taux d'augmentation des prix à la consommation des denrées alimentaires ont eu lieu en Argentine et en Chine, et dans une moindre mesure au Brésil, au Chili et en Ukraine. Par contre, malgré la chute des cours internationaux, les prix ont plus rapidement augmenté en 2008/09 en Afrique du Sud, en Inde, en Indonésie, en Russie et au Vietnam, tout comme dans la zone de l'OCDE considérée globalement. 
Tableau 2.4. Taux de variation annuel moyen des prix à la consommation des denrées alimentaires 2003/04-2008/09

\begin{tabular}{|c|c|c|c|c|c|c|}
\hline \multirow[b]{2}{*}{ Pays } & \multicolumn{6}{|c|}{ Année finissant en juin, \% } \\
\hline & $2003 / 04$ & $2004 / 05$ & $2005 / 06$ & $2006 / 07$ & $2007 / 08$ & $2008 / 09$ \\
\hline Argentine & 5.0 & 7.5 & 13.6 & 10.9 & 9.3 & 3.8 \\
\hline Brésil & 9.7 & 4.4 & 0.6 & 2.4 & 11.1 & 10.6 \\
\hline Chili & -0.3 & -0.4 & 4.4 & 2.6 & 17.0 & 12.5 \\
\hline Chine & 7.2 & 7.2 & 1.7 & 5.2 & 18.7 & 4.1 \\
\hline Inde & 2.9 & 2.2 & 6.4 & 10.4 & 8.7 & 12.5 \\
\hline Indonésie & 2.4 & 7.0 & 15.3 & 11.6 & 13.5 & 14.0 \\
\hline Russie & 10.0 & 12.5 & 10.9 & 7.7 & 15.9 & 16.4 \\
\hline $\begin{array}{l}\text { Afrique du } \\
\text { Sud }\end{array}$ & 2.8 & 1.0 & 3.6 & 7.7 & 13.3 & 15.0 \\
\hline Ukraine & 9.4 & 15.8 & 10.7 & 2.4 & 29.0 & 21.9 \\
\hline Vietnam & 4.9 & 14.2 & 9.3 & 8.3 & 24.0 & 25.9 \\
\hline OCDE & 3.0 & 2.1 & 1.3 & 3.1 & 4.9 & 5.2 \\
\hline
\end{tabular}

Source : Base statistique de l'OCDE et organismes statistiques nationaux.

Les hausses des prix à la consommation des denrées alimentaires ont exercé des pressions à la hausse de l'inflation dans le monde entier, en particulier dans les économies en développement où l'alimentation représente une part importante de l'ensemble de la consommation. Leur impact sur l'inflation est en effet d'autant plus marqué que l'alimentation occupe une large place dans le budget des ménages. Dans la plupart des pays développés, la part des dépenses alimentaires représente de $10 \%$ à $20 \% .{ }^{10}$ Dans les dix pays émergents, la part des dépenses alimentaires dans le budget des ménages est en règle générale beaucoup plus élevée. L'alimentation y contribue donc à l'inflation pour une plus large part, allant d'environ $20 \%$ en Afrique du Sud, au Brésil, au Chili et en Indonésie jusqu'à 55\% en Ukraine (tableau 2.5).

Comme il fallait s'y attendre compte tenu de l'analyse ci-dessus, l'inflation s'est considérablement accrue du fait de la hausse des prix des denrées alimentaires dans tous les pays en 2006/07-07/08 par rapport à 2003/04-05/06 (graphique 2.13 et tableau 2.5). Du fait en grande partie d'un haut degré de transmission des prix, l'Afrique du Sud et le Chili se sont caractérisés par les plus fortes augmentations de la contribution de la hausse des prix des denrées alimentaires à l'inflation entre ces deux périodes. Le niveau de cette contribution est toutefois demeuré plus faible que dans la plupart des autres pays du fait en partie du moindre poids relatif accordé aux produits alimentaires dans les indices de l'inflation, conséquence de la part relative plus réduite de l'alimentation dans les dépenses des ménages (tableau 1.5). Par contre, cette contribution atteint les niveaux les plus élevés en Argentine, en Chine, en Inde, en Russie, en Ukraine et au Vietnam. Dans ces pays, la part de l'alimentation dans les dépenses de consommation et le poids attaché aux produits alimentaires dans les indices de l'inflation sont relativement sont relativement élevés.

10. C'est pourquoi l'indice des prix des denrées alimentaires dans la zone de l'OCDE est supposé représenter $15 \%$ de l'IPC pour l'ensemble des pays de l'OCDE. 
Graphique 2.13. Augmentation annuelle moyenne de l'inflation imputable à la hausse des produits alimentaires, 2003/04-05/06 et 2006/07-07/08

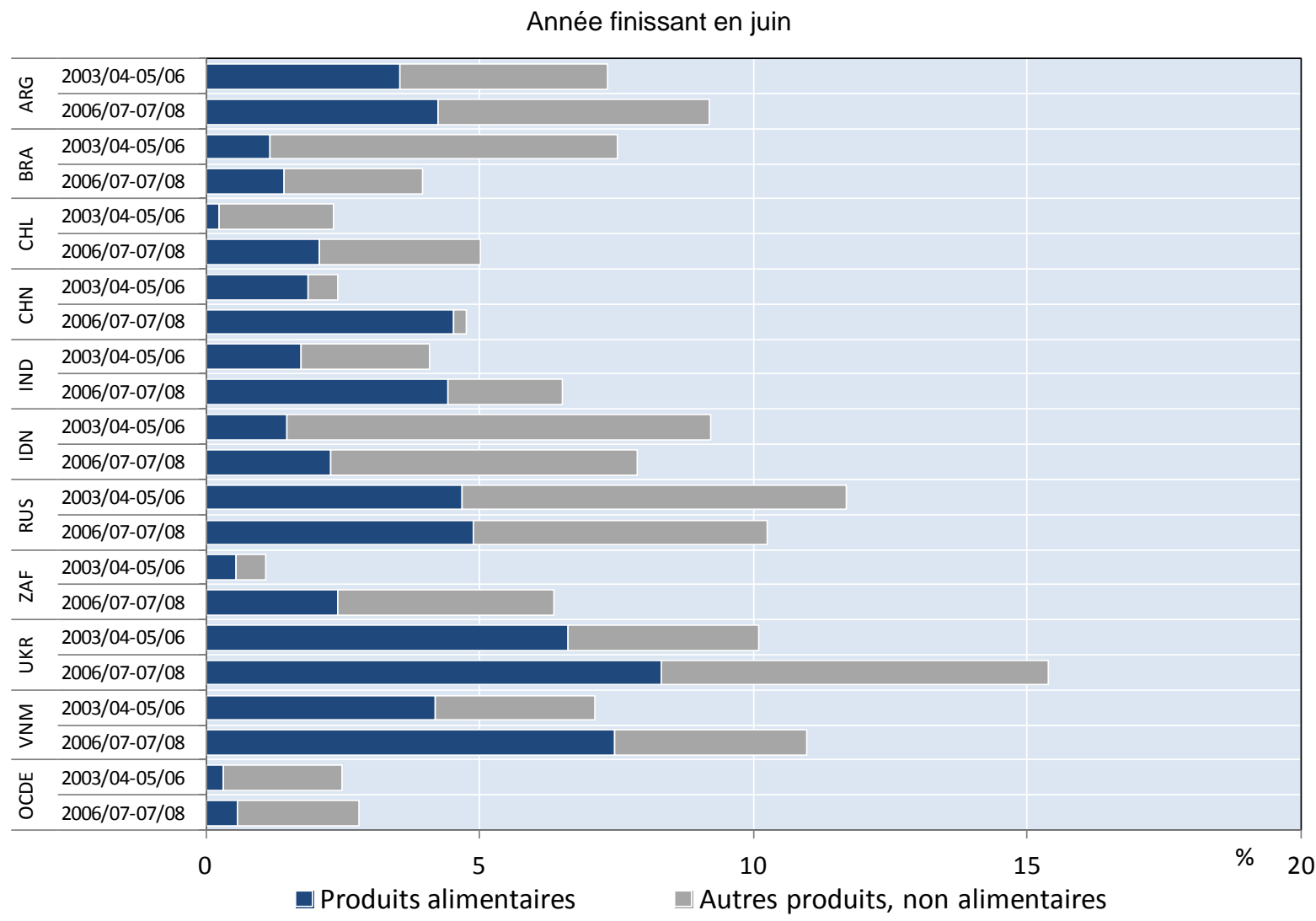

Note: L'augmentation de l'inflation attribuée à la hausse des prix des denrées alimentaires est obtenue en multipliant le taux d'accroissement des prix alimentaires par le poids accordé à l'alimentation dans l'indice de l'inflation. La contribution des produits autres que les denrées alimentaires correspond à la différence entre le taux d'inflation global et celui attribué à l'alimentation. La somme des barres empilées est égale au taux d'inflation global pour cette période.

Source : Base statistique de l'OCDE et organismes statistiques nationaux.

Tableau 2.5. Augmentation annuelle moyenne de l'inflation imputable à la hausse des produits alimentaires, 2003/04-2008/09

\begin{tabular}{l|c|cccccc}
\hline & Poids dans & \multicolumn{5}{|c}{ Année finissant en juin, \% } \\
\cline { 3 - 7 } Pays & l'IPC, \% & $\mathbf{2 0 0 3 / 0 4}$ & $\mathbf{2 0 0 4 / 0 5}$ & $\mathbf{2 0 0 5 / 0 6}$ & $\mathbf{2 0 0 6 / 0 7}$ & $\mathbf{2 0 0 7 / 0 8}$ & $\mathbf{2 0 0 8 / 0 9}$ \\
\hline Argentine & 36 & 2.0 & 3.1 & 5.6 & 4.5 & 4.0 & 1.6 \\
Brésil & 22 & 2.4 & 1.0 & 0.2 & 0.5 & 2.4 & 2.4 \\
Chili & 22 & -0.1 & -0.1 & 0.9 & 0.6 & 3.6 & 2.9 \\
Chine & 33 & 2.4 & 2.6 & 0.6 & 1.9 & 7.1 & 1.8 \\
Inde & 46 & 1.3 & 1.0 & 2.9 & 4.7 & 4.1 & 6.1 \\
Indonésie & 20 & 0.4 & 1.3 & 2.7 & 2.1 & 2.5 & 2.8 \\
Russie & 44 & 4.3 & 5.2 & 4.6 & 3.2 & 6.5 & 7.1 \\
Afrique du & 21 & 0.6 & 0.2 & 0.8 & 1.7 & 3.1 & 3.7 \\
Sud & & & & & & 1.5 & 15.2 \\
Ukraine & 55 & 5.1 & 8.6 & 6.2 & 1.5 & 12.8 \\
Vietnam & 43 & 2.1 & 6.3 & 4.3 & 3.8 & 11.1 & 13.6 \\
\hline OCDE & 15 & 0.4 & 0.3 & 0.2 & 0.4 & 0.7 & 0.8 \\
\hline
\end{tabular}

Source : Base statistique de l'OCDE et organismes statistiques nationaux.

Un autre indicateur peut aider à montrer à quel point les consommateurs ont été affectés par la hausse des prix des denrées alimentaires. Il s'agit de l'évolution relative des prix des denrées alimentaires et des 
autres produits, non alimentaires. Cette évolution peut être examinée en mesurant la variation du rapport entre les prix alimentaires et non alimentaires (ces derniers étant calculés en soustrayant la part des produits alimentaires dans l'indice de l'inflation). Des taux négatifs indiquent une baisse relative des prix alimentaires par rapport aux prix non alimentaires dans le pays considéré au cours de la période envisagée ; des taux positifs indiquent au contraire une augmentation relative. Lorsque les prix alimentaires augmentent, un taux négatif montre qu'ils ont connu une hausse plus lente que les prix non alimentaires, alors qu'un taux positif met au contraire en évidence leur progression plus rapide.

Au cours de la période triennale 2003/04-05/06, dans cinq des économies émergentes (Brésil, Chili, Inde, Indonésie et Russie), tout comme dans l'ensemble de la zone de l'OCDE, les prix alimentaires ont augmenté plus lentement que les prix non alimentaires (graphique 2.14 et tableau 2.6). Dans les cinq autres pays (Afrique du Sud, Argentine, Chine, Ukraine et Vietnam) les prix alimentaires ont augmenté plus vite que les prix non alimentaires.

\section{Graphique 2.14. Taux de variation annuel moyen des prix à la consommation des denrées alimentaires - 2003/04-05/06 et 2006/07-07/08}

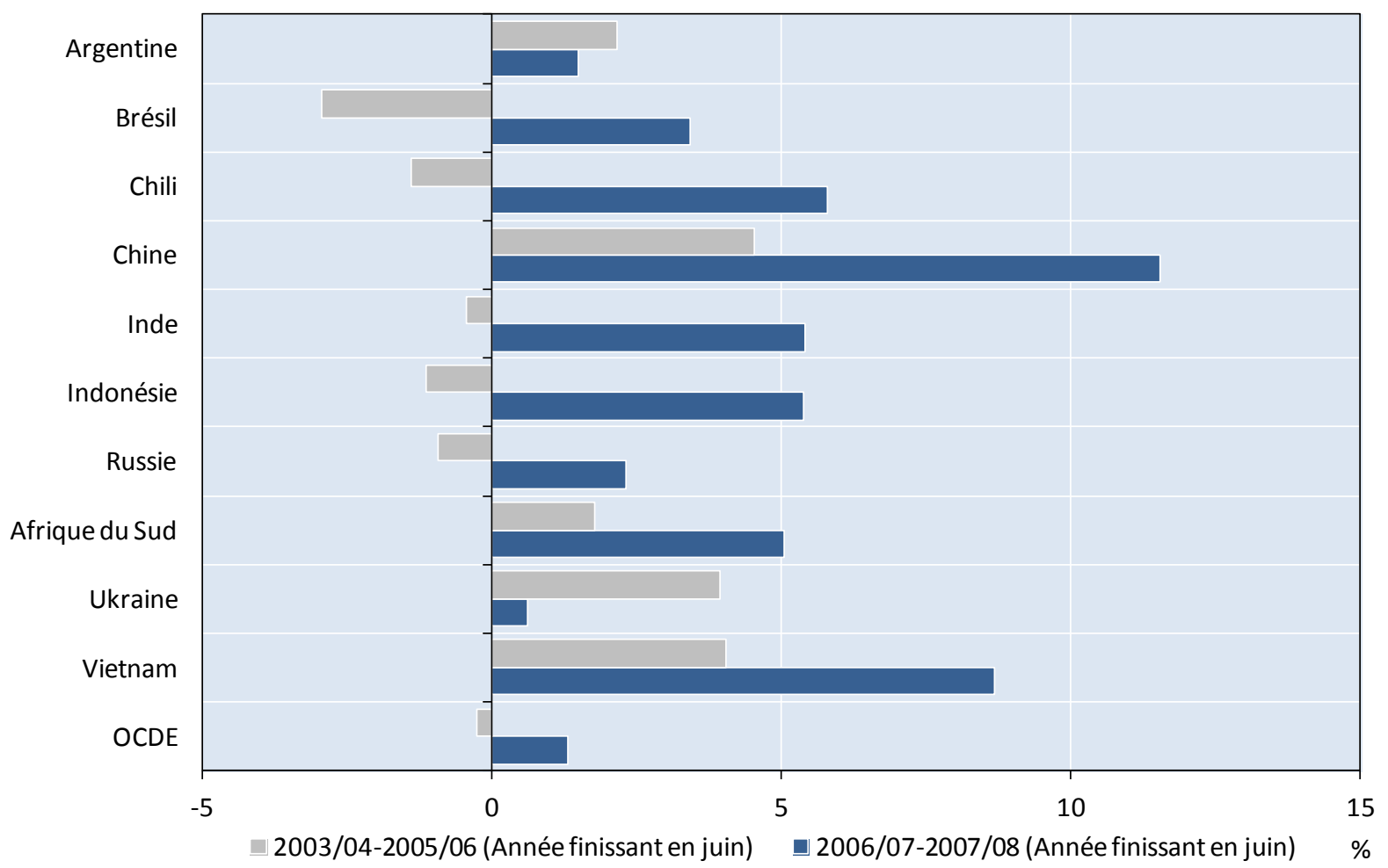

Source : Base statistique de l'OCDE et organismes statistiques nationaux.

Par contre, au cours de la période biennale 2006/07-07/08, les prix alimentaires ont augmenté plus vite que les prix non alimentaires dans chacun des dix pays. Dans tous les cas, exception faite de l'Argentine et de l'Ukraine, le taux d'augmentation des prix alimentaires a été sensiblement supérieur à celui des prix non alimentaires. Bien que les prix alimentaires aient progressé plus vite que les prix non alimentaires en Ukraine comme en Argentine, leurs taux d'augmentation respectifs étaient plus proches en 2006/07-07/08 qu'en 2003/04-05/06. En Argentine, cela peut en partie tenir aux subventions accordées aux transformateurs de céréales et de lait, financées grâce à une augmentation des taxes à l'exportation. En Ukraine, la baisse des prix alimentaires par rapport aux prix non alimentaires en 2006/07 a été le résultat d'une sensible augmentation des prix administrés du gaz. La plupart des pays ont connu une baisse du taux d'augmentation des prix alimentaires par rapport à celui des prix non alimentaires en 2008/09, sauf 
l'Afrique du Sud, l'Inde et le Vietnam, où il s'est accru. Les prix alimentaires ont également continué d'augmenter plus vite que les prix non alimentaires dans l'ensemble des pays de l'OCDE.

Tableau 2.6. Taux de variation annuel moyen des prix alimentaires par rapport aux prix non alimentaires 2003/04-2008/09

\begin{tabular}{l|cccccc}
\hline & \multicolumn{7}{|c}{ Année finissant en juin } \\
\cline { 2 - 7 } Pays & $\mathbf{2 0 0 3 / 0 4}$ & $\mathbf{2 0 0 4 / 0 5}$ & $\mathbf{2 0 0 5 / 0 6}$ & $\mathbf{2 0 0 6 / 0 7}$ & $\mathbf{2 0 0 7 / 0 8}$ & $\mathbf{2 0 0 8 / 0 9}$ \\
\hline Argentine & 1.9 & 0.8 & 3.8 & 1.8 & 1.1 & -5.3 \\
Brésil & 0.4 & -3.6 & -5.9 & -1.2 & 8.0 & 5.9 \\
Chili & -1.7 & -3.3 & 0.8 & -0.2 & 11.8 & 7.4 \\
Chine & 6.8 & 6.2 & 0.5 & 4.4 & 18.7 & 4.8 \\
Inde & -0.8 & -3.3 & 2.7 & 6.9 & 3.9 & 5.8 \\
Indonésie & -4.0 & -0.2 & 0.6 & -1.3 & 5.0 & 5.7 \\
Russie & -2.6 & 0.1 & -0.2 & 3.6 & 6.5 & 4.4 \\
Afrique du & 3.8 & -0.2 & 1.7 & & 6.9 \\
Sud & & & & -14.6 & 15.5 & 3.1 \\
Ukraine & 4.4 & 6.9 & 0.4 & 2.3 & 15.2 & 1.9 \\
Vietnam & 1.1 & 8.6 & 2.3 & 0.8 & & 3.6 \\
\hline OCDE & 0.9 & -0.5 & -1.7 & & \\
\hline
\end{tabular}

Source : Base statistique de l'OCDE et organismes statistiques nationaux.

Outre la hausse des cours mondiaux, des facteurs intérieurs tels que des conditions météorologiques défavorables ont contribué à l'acuité de la hausse des prix des denrées alimentaires en 2007/08, en particulier au Chili, en Chine, en Ukraine et au Vietnam. L'hiver 2007 (juin-août) a été l'un des plus rudes qu'ait connus le Chili et il a été suivi par une période de sécheresse au cours de l'été 2007/08. Cela a eu un impact très négatif sur la production de fruits et légumes. Les tempêtes de neige les plus violentes depuis 40 ans se sont abattues sur la Chine début 2008. De nombreuses cultures ont été détruites, notamment de fruits et légumes. Les délais dans le transport ont entraîné des pénuries de denrées alimentaires et de fortes augmentations des prix. C'était là une conséquence de la prolifération des cas de syndrome dysgénésique et respiratoire du porc (maladie des oreilles bleues) en mai 2007. Quarante millions de porcs ont été perdus, et les prix de la viande de porc ont augmenté de 45\%. Cela explique l'augmentation sensible des les prix alimentaires par rapport aux prix non alimentaires en Chine en 2007/08 malgré les politiques isolant le marché intérieur des hausses des prix des céréales.

Des conditions météorologiques défavorables en Ukraine ont entraîné une mauvaise récolte de céréales, de fruits et de légumes entre la mi-2007 et la mi-2008. Une forte augmentation des dépenses publiques de sécurité sociale (pensions de retraite et salaires de subsistance) et un relèvement du salaire minimum ont exacerbé la hausse des prix des denrées alimentaires en 2007/08 du fait de la part importante de l'alimentation, de l'habillement et des chaussures dans la consommation des ménages à faible revenu. Au Vietnam, la production agricole et horticole a été victime en 2007 et en 2008 de conditions météorologiques défavorables, dont des sécheresses et inondations, ainsi que d'une prolifération de ravageurs et de maladies des cultures, et plus particulièrement d'infestations par la cicadelle brune du riz et de maladies foliaires dans le delta du Mékong. Des épizooties telles que la grippe aviaire ou la maladie des oreilles bleues ont perturbé la production du secteur de l'élevage.

\subsection{Consommation}

Il serait certes utile d'analyser les évolutions dans la composition de la consommation par habitant de produits alimentaires, mais les données d'enquêtes sur la consommation récentes ne sont pas aisément accessibles. Des estimations peuvent être établies à l'aide de méthodes fondées sur l'offre et l'utilisation des produits mais des sources d'incertitude majeures entourent ces données. Une autre solution consiste à estimer l'impact monétaire sur les consommateurs de la hausse des prix des denrées alimentaires. L'impact 
sur les consommateurs dépend de l'évolution des prix des denrées alimentaires par rapport à ceux des autres produits, l'importance de l'alimentation dans la consommation, mais aussi jusqu'à quel point les consommateurs peuvent aisément procéder à des substitutions entre la consommation de denrées alimentaires et celle d'autres produits de nature non alimentaire.

La variation compensatoire (VC) mesure la variation des revenus ou des dépenses monétaires nécessaire pour maintenir un niveau d'utilité constant après une modification des prix relatifs. Cette méthode a été appliquée dans plusieurs études empiriques récentes pour mesurer l'effet des variations des prix des denrées alimentaires sur les consommateurs (Ackah et Appleton, 2007 ; Dessus et al., 2008 ; et Leyaro, 2009). Il est possible de démontrer que la CV peut être estimée sur la base des variations en pourcentage et des parts dans le budget des ménages, à l'aide de la formule suivante :

$$
\ln \Delta c \approx w \Delta \ln p+\frac{1}{2} w \Delta \ln p \varepsilon
$$

où :

$\ln \Delta c$ correspond à la variation en pourcentage des dépenses de consommation ;

$w$ représente la part de l'alimentation dans les dépenses de consommation ;

$\Delta \ln p$ est la variation en pourcentage du prix des denrées alimentaires par rapport à celui des autres produits ; et

$\varepsilon$ est l'élasticité du prix des denrées alimentaires par rapport à celui des autres produits.

Le second élément de l'équation rend compte des effets de substitution, c'est-à-dire de la variation des quantités demandées à la suite d'une modification des prix relatifs. Les éléments indispensables pour estimer la CV sont : (a) la variation des prix relatifs, (b) la part de la consommation de denrées alimentaires dans le budget total des ménages et (c) l'élasticité de substitution entre les denrées alimentaires et les produits non alimentaires. Le premier de ces éléments est issu des travaux examinés dans la section précédente, consacrée à la hausse des denrées alimentaires en termes réels. Le second élément peut être tiré des statistiques de sécurité alimentaire de la FAO. Les élasticités des prix peuvent être trouvées dans les travaux réalisés par l'USDA (Seale et al., 2003). Afin de maintenir la cohérence avec l'analyse précédemment menée, le tableau 2.7 présente les résultats des variations de prix survenues au cours des deux périodes triennales débutant respectivement en 2002/03 et en 2005/06. Ce tableau montre combien il importe de prendre en considération d'autres facteurs que la seule hausse des prix des denrées alimentaires pour déterminer l'impact de celle-ci sur les consommateurs. 
Tableau 2.7. Estimation de la variation compensatoire entre 2002/03-05/06 et 2005/06-08/09

\begin{tabular}{|c|c|c|c|c|c|c|c|c|}
\hline \multirow[b]{3}{*}{ Pays } & \multirow{3}{*}{$\begin{array}{c}\text { Part de } \\
\text { l'alimentation } \\
\text { dans les } \\
\text { dépenses de } \\
\text { consommation } \\
\text { des ménages }\end{array}$} & \multirow{3}{*}{$\begin{array}{c}\text { Élasticité } \\
\text { du prix des } \\
\text { denrées } \\
\text { alimentaires } \\
\text { par rapport } \\
\text { aux autres } \\
\text { produits }\end{array}$} & \multicolumn{6}{|c|}{ Année finissant en juin } \\
\hline & & & \multicolumn{3}{|c|}{$\begin{array}{c}\text { Variation relative du prix des } \\
\text { denrées alimentaires par rapport } \\
\text { aux autres produits, \% }\end{array}$} & \multicolumn{3}{|c|}{ Variation compensatoire, $\%$} \\
\hline & & & $\begin{array}{c}2002 / 03- \\
2005 / 06\end{array}$ & $\begin{array}{c}2005 / 06- \\
2007 / 08\end{array}$ & $\begin{array}{c}2005 / 06 /- \\
2008 / 09\end{array}$ & $\begin{array}{c}2002 / 03- \\
2005 / 06\end{array}$ & $\begin{array}{c}2005 / 06- \\
2007 / 08\end{array}$ & $\begin{array}{c}2005 / 06- \\
2008 / 09\end{array}$ \\
\hline Argentine & 33 & -0.357 & 6.6 & 2.9 & -2.6 & 1.8 & 0.8 & -0.7 \\
\hline Brésil & 21 & -0.391 & -8.8 & 6.7 & 13.0 & -1.5 & 1.1 & 2.2 \\
\hline Chili & 23 & -0.383 & -4.2 & 11.5 & 19.8 & -0.8 & 2.1 & 3.6 \\
\hline Chine & 40 & -0.390 & 13.9 & 23.9 & 29.8 & 4.5 & 7.7 & 9.6 \\
\hline Inde & 50 & -0.390 & -1.5 & 11.0 & 17.4 & -0.6 & 4.4 & 7.0 \\
\hline Indonésie & 48 & -0.391 & -3.5 & 11.0 & 17.3 & -1.4 & 4.3 & 6.7 \\
\hline Russie & 33 & -0.390 & -2.8 & 4.5 & 9.1 & -0.7 & 1.2 & 2.4 \\
\hline $\begin{array}{l}\text { Afrique du } \\
\text { Sud }\end{array}$ & 25 & -0.390 & 5.3 & 10.3 & 18.0 & 1.1 & 2.1 & 3.6 \\
\hline Ukraine & 61 & -0.393 & 12.0 & -1.4 & 1.7 & 5.9 & -0.7 & 0.8 \\
\hline Vietnam & 51 & -0.372 & 12.3 & 17.9 & 37.0 & 5.1 & 7.4 & 15.3 \\
\hline OCDE & 15 & -0.270 & -1.2 & 2.6 & 6.3 & -0.2 & 0.3 & 0.8 \\
\hline
\end{tabular}

Dans tous les pays (y compris ceux de l'OCDE) sauf l'Ukraine, le prix relatif des denrées alimentaires par rapport aux autres produits a augmenté entre l'année finissant en juin 2006 et celle finissant en juin 2008. Cette tendance à la hausse s'est poursuivie entre 2008 et 2009 dans tous les pays à l'exception de l'Argentine, où le prix relatif des denrées alimentaires a baissé à tel point qu'il était plus faible en 2009 qu'en 2006. Aussi la VC estimée a-t-elle augmenté entre ces deux périodes dans tous les pays sauf l'Argentine. Les pays où l'alimentation représente une part importante des dépenses de consommation sont les plus durement touchés, comme par exemple ceux d'Asie. Les consommateurs indiens et ceux d'Afrique du Sud ont ainsi connu une évolution très similaire des prix relatifs des denrées alimentaires entre 2005/06 et 2007/08. L'élasticité de substitution par rapport aux prix entre les denrées alimentaires et les autres produits est comparable pour les premiers comme pour les seconds. L'alimentation représente toutefois $50 \%$ de la consommation des ménages en Inde, contre seulement $25 \%$ en Afrique du Sud. Il s'ensuit que l'augmentation des dépenses de consommation nécessaire pour maintenir la même utilité en Inde est deux fois plus élevée qu'en Afrique du Sud.

Après avoir calculé la variation en pourcentage des dépenses de consommation nécessaires pour maintenir le même niveau d'utilité d'une période sur l'autre à la suite d'une évolution des prix relatifs entre les denrées alimentaires et les autres produits, il pourrait être intéressant de comparer la VC estimée aux variations de revenus générées par la croissance générale de l'économie. La hausse des prix alimentaires a sans nul doute défavorisé les consommateurs, mais dans quelle mesure la croissance parallèle des revenus a-t-elle atténué le coût imposé par le renchérissement des denrées alimentaires ? Malheureusement, les données relatives aux variations des niveaux de revenus des ménages ne sont pas aisément accessibles. Aussi une autre méthode reposant sur les variations du PIB moyen par habitant exprimé en termes constants a-t-elle été appliquée. 
Tableau 2.8. Variation compensatoire et variations du PIB réel par habitant, 2005/06-2007/08 et 2005/06-2008/09

\begin{tabular}{l|cc|cc}
\hline \multirow{2}{*}{ Pays } & \multicolumn{2}{|c|}{ Variation entre 2005/06 et 2007/08 } & \multicolumn{2}{c}{ Variation entre 2005/06 et 2008/09 } \\
\cline { 2 - 5 } & $\begin{array}{c}\text { Variation } \\
\text { compensatoire }\end{array}$ & $\begin{array}{c}\text { PIB par } \\
\text { habitant, en } \\
\text { termes constants }\end{array}$ & $\begin{array}{c}\text { Variation } \\
\text { compensatoire }\end{array}$ & $\begin{array}{c}\text { PIB par habitant, en } \\
\text { termes constants }\end{array}$ \\
\hline Argentine & 0.8 & 13.7 & -0.7 & 9.8 \\
Brésil & 1.1 & 8.7 & 2.2 & 6.9 \\
Chili & 2.1 & 5.8 & 3.6 & 31.7 \\
Chine & 7.7 & 22.0 & 9.6 & 18.4 \\
Inde & 4.4 & 14.0 & 7.0 & 12.8 \\
Indonésie & 4.3 & 9.8 & 6.7 & 6.6 \\
Russie & 1.2 & 14.8 & 2.4 & 2.0 \\
Afrique du & 2.1 & 5.4 & 3.6 & -3.4 \\
Sud & -0.7 & 11.4 & 0.8 & 16.2 \\
\hline Ukraine & 7.4 & 12.5 & 15.3 & \\
\hline Vietnam & & & & \\
\hline
\end{tabular}

Source : Tableau 2.7 et FMI, Base de données des statistiques financières internationales (2010).

Dans chacun des dix pays, la croissance du PIB réel par habitant entre 2005/06 et 2007/08 a été supérieure à l'augmentation des dépenses de consommation estimée nécessaire pour maintenir le même niveau d'utilité (tableau 2.8). Il en était même ainsi pour la Chine et le Vietnam, où l'augmentation des dépenses nécessaire était pourtant la plus forte. Il en va néanmoins tout autrement lorsque la période étudiée est prolongée de manière à inclure l'année 2008/09. En Afrique du Sud, au Chili et en Ukraine, les variations du PIB réel par habitant entre 2005/06 et 2008/09 ont été inférieures à l'augmentation des dépenses de consommation nécessaires pour maintenir constante l'utilité dont jouissent les consommateurs. Dans tous les pays à l'exception de la Chine et de l'Inde, l'écart entre les variations en pourcentage de ces deux variables s'est resserré entre 2005/06-07/08 et 2005/06/08/09. Par conséquent, si la forte croissance économique enregistrée jusqu'en 2007/08 a atténué les pressions sur les consommateurs générées par le renchérissement des prix des denrées alimentaires, la conjonction du ralentissement de l'augmentation du PIB imputable à la crise économique mondiale et de la poursuite de la hausse des prix des denrées alimentaires par rapport à ceux des autres produits a mis à rude épreuve les consommateurs.

\subsection{Production}

Un autre facteur doit également être pris en compte: l'ajustement de la production par les agriculteurs. Une récente analyse montre que la hausse des prix a encouragé une expansion de la production mondiale de céréales mais qu'elle s'est surtout concentrée dans les pays développés, la plupart des exploitants pauvres des pays en développement n'ayant pas saisi cette occasion (FAO, 2009c). Les graphiques 2.15 à 2.18 montre l'ajustement de la production et des superficies cultivées dans les dix économies émergentes pour les quatre produits de base considérés. Les évolutions de la production et de la superficie récoltée aux États-Unis et dans l'ensemble du monde sont également prises en compte à titre comparatif. Compte tenu des grandes différences des niveaux de production et des superficies cultivées selon les pays, ces variables sont indexées sur leur niveau moyen au cours de la période triennale 2004-06.

Les superficies et les volumes de production des trois cultures céréalières et du soja ont de manière générale augmenté dans les dix pays durant la période 2007-09. Les taux d'augmentation y ont été au moins égaux, et même bien souvent supérieurs, à ceux observés aux États-Unis et dans l'ensemble du monde. L'augmentation a été particulièrement sensible dans le cas du maïs, où il s'est produit une augmentation régulière des superficies cultivées et de la production durant ces trois années dans la quasitotalité des pays. 
Il s'est produit une forte augmentation de la production de céréales en Russie, et de celle de blé en Ukraine, du fait de la hausse des prix, des conditions météorologiques favorables et des mesures propices prises par les pouvoirs publics. Par exemple, la douceur de l'hiver début 2008, puis l'arrivée précoce du printemps et une très bonne teneur en eau du sol ont entraîné une excellente récolte de blé en 2008 . La hausse des prix a également incité les agriculteurs à consacrer davantage de superficies à la culture des céréales et les grandes sociétés céréalières à développer leurs investissements dans les terres agricoles, contribuant à une amélioration des rendements. En outre, les céréaliculteurs russes ont bénéficié en 2008 d'un surcroît de subventions en faveur des engrais et de plus grandes possibilités d'obtention de crédits bonifiés dans le cadre du Programme d'État pour le développement de l'agriculture de 2008 à 2012. La baisse des prix qui s'en est suivie en 2008/09 a entraîné une baisse des superficies emblavées. Malgré un niveau de départ extrêmement réduit, la production de riz s'est sensiblement accrue en Russie à la suite des mesures gouvernementales qui ont entraîné une hausse des prix intérieurs du riz.

L'Afrique du Sud, l'Argentine et le Chili vont à contre-courant de cette tendance pour ce qui est du blé. En Argentine, la forte baisse de la production de blé en 2008 était principalement la conséquence d'une grave sécheresse - les précipitations ont été quasiment nulles entre octobre et décembre 2008 et les températures ont atteint des niveaux très élevés - bien que les superficies cultivées aient par ailleurs diminué de 25\% par rapport à l'année précédente. En 2009, il s'est produit une nouvelle diminution des superficies ensemencées de blé, qui sont tombées à 2.8 millions d'hectares, contre 4.3 millions d'hectares en 2008. Jamais depuis plus d'un siècle les superficies emblavées n'avaient été si faibles. L'incitation à produire du blé en Argentine a été érodée par la sécheresse de 2008, par les difficultés à lever des capitaux pour acheter engrais et semences, et par les incessantes interventions des pouvoirs publics dans les échanges de blé, au moyen notamment de fortes taxes à l'exportation. Les agriculteurs se sont reportés sur les cultures oléagineuses telles que le soja et le colza dont les coûts de production sont plus faibles et dont les prix ont évolué de manière plus satisfaisante. Par exemple, environ $90 \%$ de la récolte de soja sont exportés, sous forme de graines ou de dérivés, aussi sont-ce bien plus les marchés extérieurs que le marché intérieur qui déterminent les gains des exploitants. Malgré une taxe à l'exportation plus élevée, les agriculteurs préfèrent produire du soja plutôt que du blé ou du maïs, car les gouvernements successifs se sont attachés à maintenir à un bas niveau le prix intérieur des céréales pour les consommateurs urbains. Les moindres coûts de production et la plus grande résistance aux variations climatiques poussent également les agriculteurs à privilégier la production de soja plutôt que celle de céréales.

Une évolution similaire, quoique moins marquée, est survenue en Afrique du Sud, où la production de blé cède du terrain au profit de celle de graines oléagineuses. Au Chili, des précipitations excessives lors des semailles comme au moment de la récolte se sont conjuguées à une baisse des prix du riz en 2006, provoquant une baisse sensible des superficies et de la production de blé en 2007. Malgré une extension en 2008 et 2009, les superficies de blé demeurent en-dessous de leur niveau de 2004-06. Les hausses des prix des carburants et des engrais ont amené les exploitants de certaines des principales régions céréalières à se tourner vers des cultures imposant de moindres coûts et comportant moins de risques, telles que l'avoine.

En général, les superficies et le volume de la production de céréales ont régulièrement augmenté dans les pays asiatiques. D'après les estimations, l'Inde a probablement connu une baisse de la récolte de riz en 2009 du fait de l'arrivée tardive de la mousson. Outre une légère augmentation des superficies consacrées à la culture du riz, les rendements ont enregistré une nette amélioration. Par exemple, la récolte de riz paddy de l'Indonésie a atteint en 2009 un niveau sans précédent de 63.84 millions de tonnes (équivalant à 40.22 millions de tonnes de riz blanchi). Cela représente une progression de $6 \%$ par rapport à la récolte exceptionnelle de l'année précédente. L'amélioration des rendements a été favorisée depuis 2007 par une plus large utilisation de semences de haute qualité dans le cadre du programme de programme, passant de 4.6 tonnes/ha en 2004-06 à près de 5 tonnes/ha en 2009. L'augmentation des superficies consacrées à la culture de riz au Vietnam a été moindre que l'on aurait pu s'y attendre de la part d'un grand pays exportateur, conséquence peut-être des incertitudes concernant la commercialisation créées par la politique 
mise en œuvre par les pouvoirs publics. Contrairement à ce qui a pu être observé dans la plupart des autres pays, la production de soja a cédé du terrain en Chine. Les prix du soja sont en Chine plus étroitement liés aux variations des cours mondiaux que ceux des céréales, et leur augmentation a donc été proportionnelle à celle des prix en vigueur sur le marché mondial en 2006-08, aussi cette réduction des quantités récoltées doit-elle sans doute être attribuée à des politiques de soutien public axées sur le développement de la production de céréales.

Un autre pays a également connu un recul des superficies de soja : le Brésil, qui a également enregistré une diminution des superficies consacrées à la culture de riz. Cela n'est pas lié aux mesures prises par le gouvernement brésilien mais s'explique plutôt en partie par l'augmentation des rendements des deux cultures sur la période considérée. L'un des principaux facteurs de l'amélioration des rendements du riz tient à la généralisation du recours au système Clearfield pour lutter contre les mauvaises herbes. Les rendements du soja ont augmenté à la suite d'une réduction des épidémies de rouille du soja, qui est ellemême une conséquence de l'adoption par les États de lois interdisant aux exploitants de planter du soja pendant une période de jachère de 90 jours afin d'empêcher la rouille du soja de se répandre en contresaison. L'amélioration des rendements a permis aux agriculteurs d'accroître les superficies consacrées à la production de maïs. 
Graphique 2.15. Évolution de la production et des superficies récoltées de blé depuis 2004-06
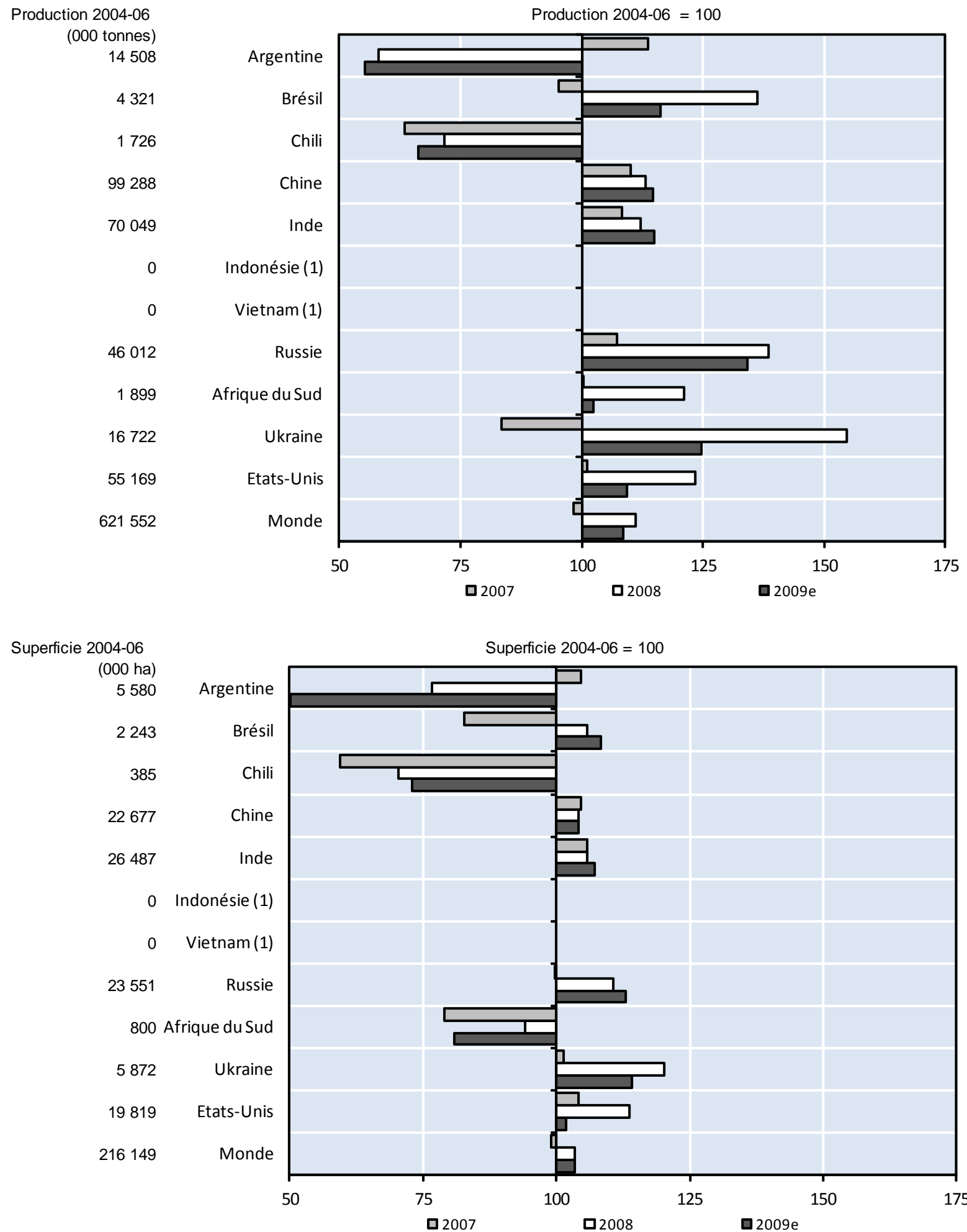

e : estimation.

1. Production commerciale négligeable. Les superficies consacrées à la production de blé en Russie en 2007 étaient très proches de la moyenne 2004-06.

Source : FAOSTAT (2010), Conseil international des céréales (2010) et informations issues de sources nationales et des rapports du Réseau d'information sur l'agriculture mondiale (Global Agriculture Information Network - GAIN) de I'USDA. 
Graphique 2.16. Évolution de la production et des superficies récoltées de maïs depuis 2004-06

Production 2004-06

(000 tonnes)

16626

39854

1403

140555

14660

11786

3691

3412

9454 Afrique du Sud

7486

283242

716479

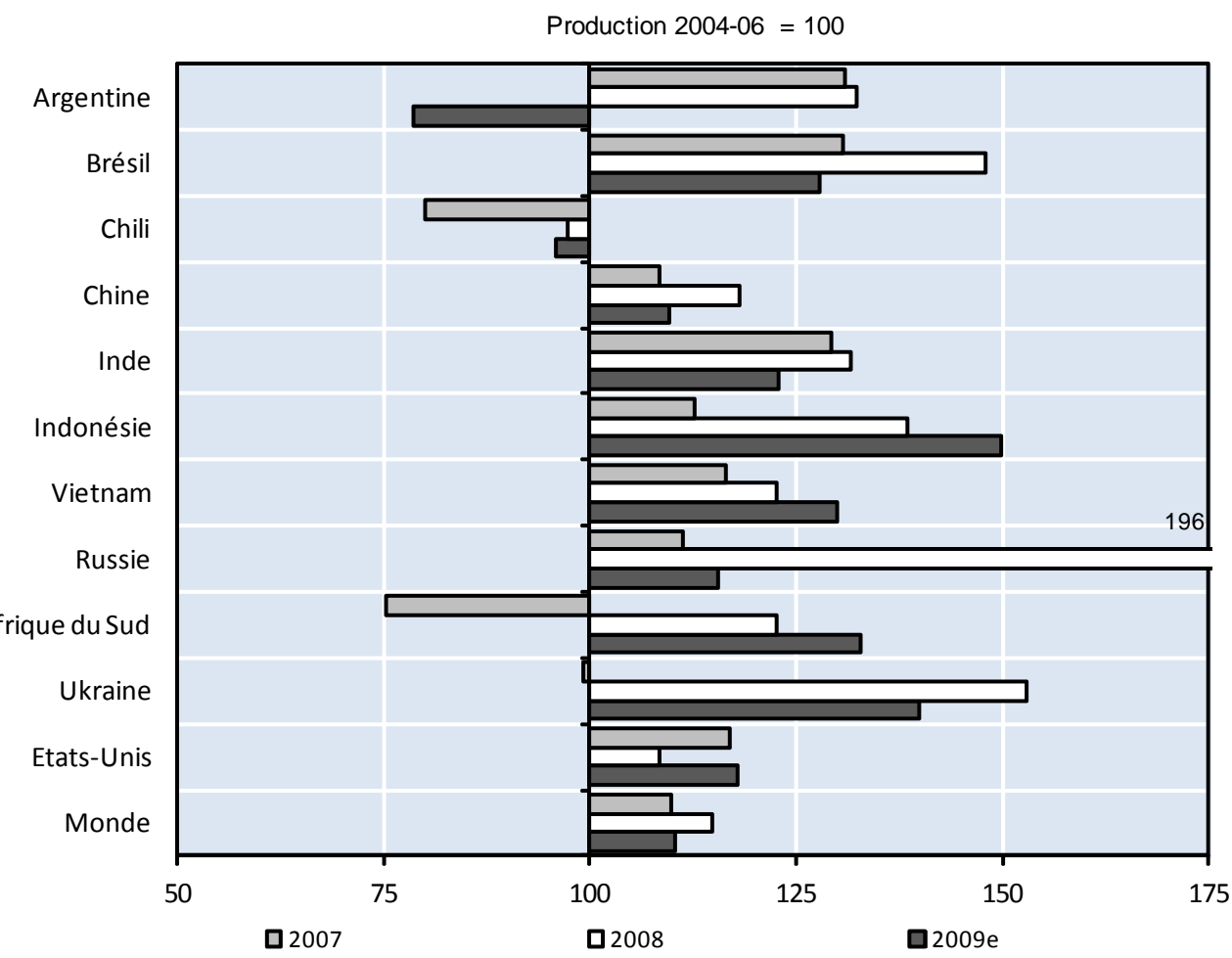

Superficie 2004-06 $=100$

(000 ha)

2523

12191

126

26776

7638

3443

1026

891

Argentine

Brésil

Chili

Chine

Inde

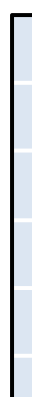

Indonésie

Vietnam

Russie

2820 Afrique du Sud

1893

29595

147841

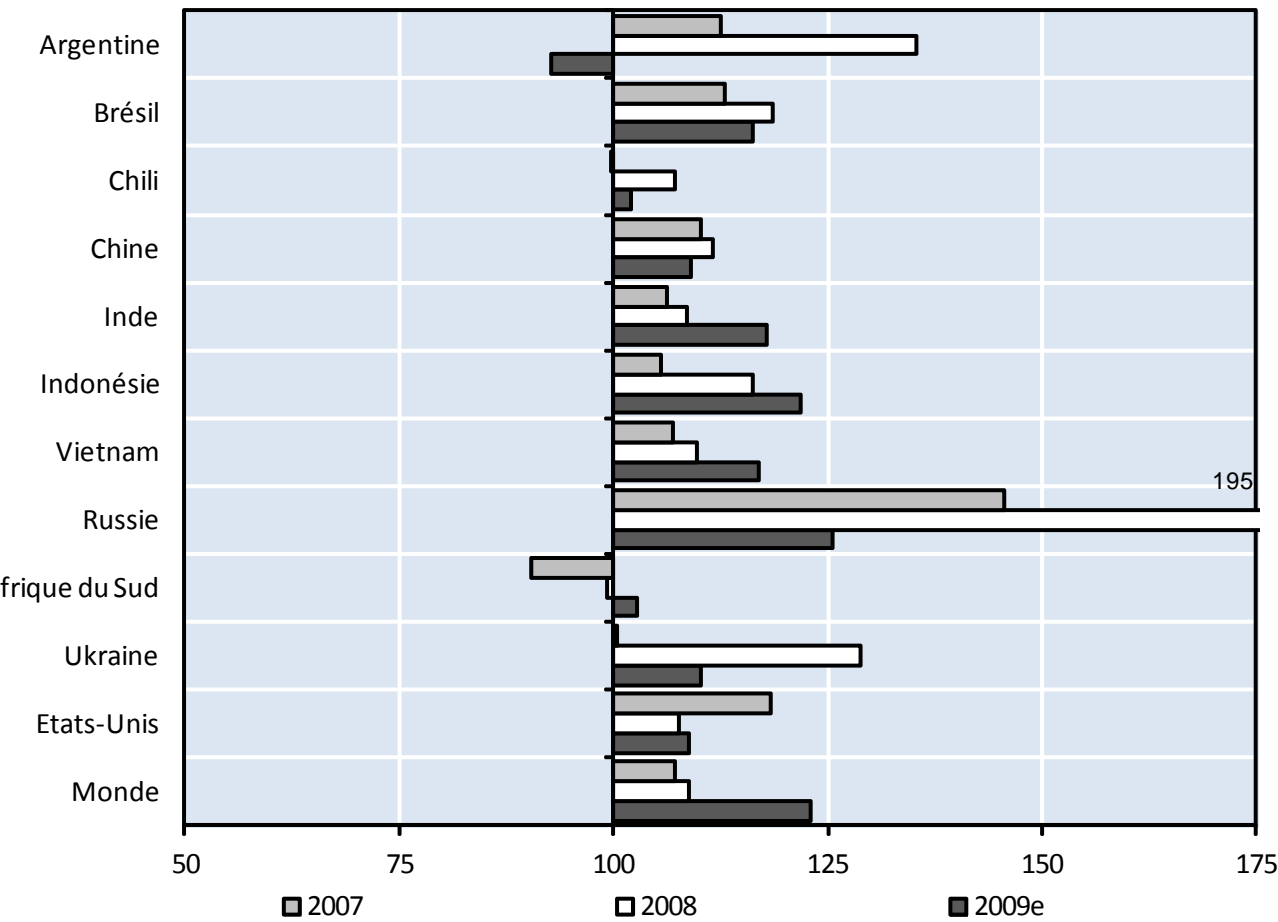

e : estimation.

Note: Les superficies consacrées à la production de maïs au Chili et en Ukraine en 2007 étaient très proches de la moyenne 2004-06.

Source : FAOSTAT (2010), Conseil international des céréales (2010) et informations issues de sources nationales et des rapports du Réseau d'information sur l'agriculture mondiale (Global Agriculture Information Network - GAIN) de l'USDA. 
Graphique 2.17. Évolution de la production et des superficies récoltées de riz depuis 2004-06

Production 2004-06

(000 tonnes)

1070

12666

132

181951

133841

54232

35944

575

0 Afrique du Sud (1)

91

9831

627832

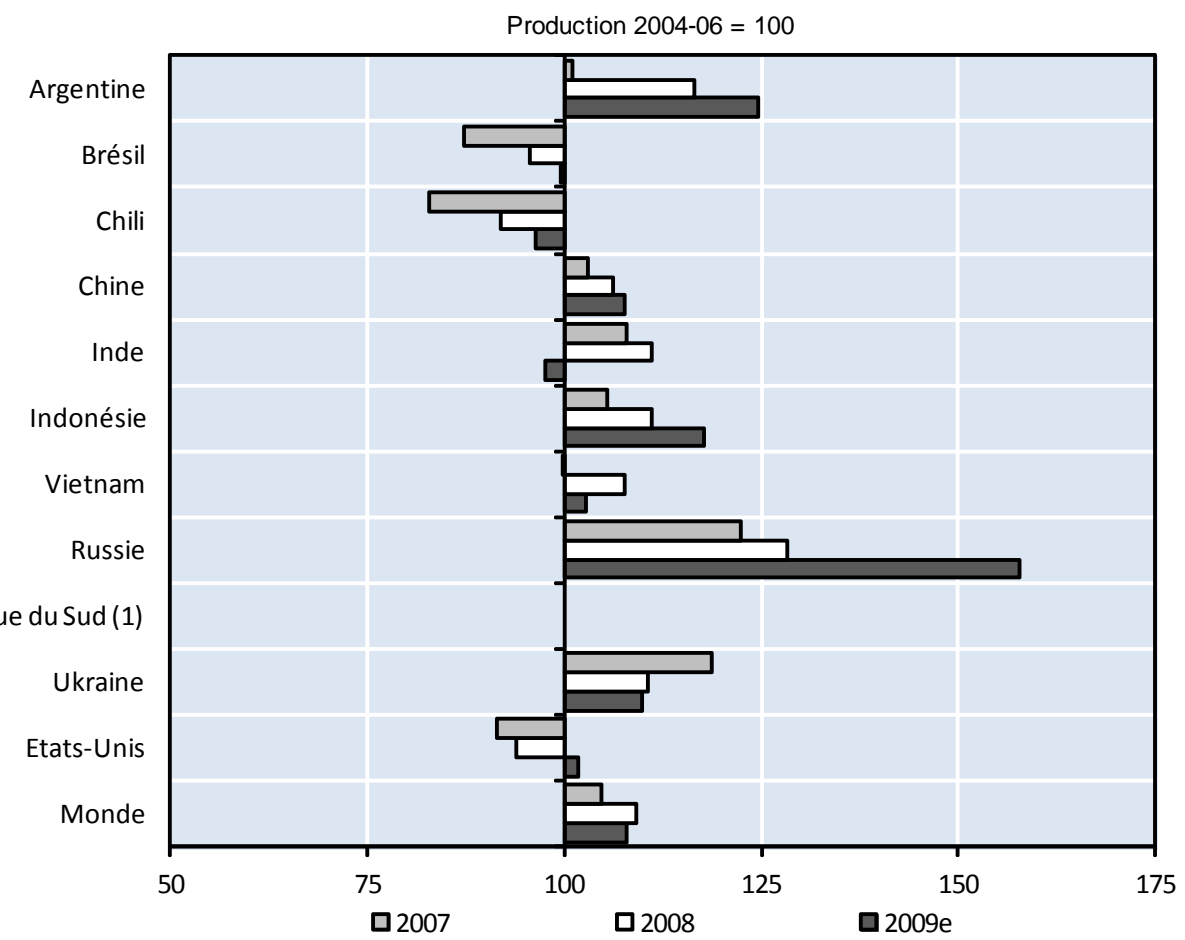

Superficie 2004-06

(000 ha)

166

3540

26

28978

43126

11849

7366

139
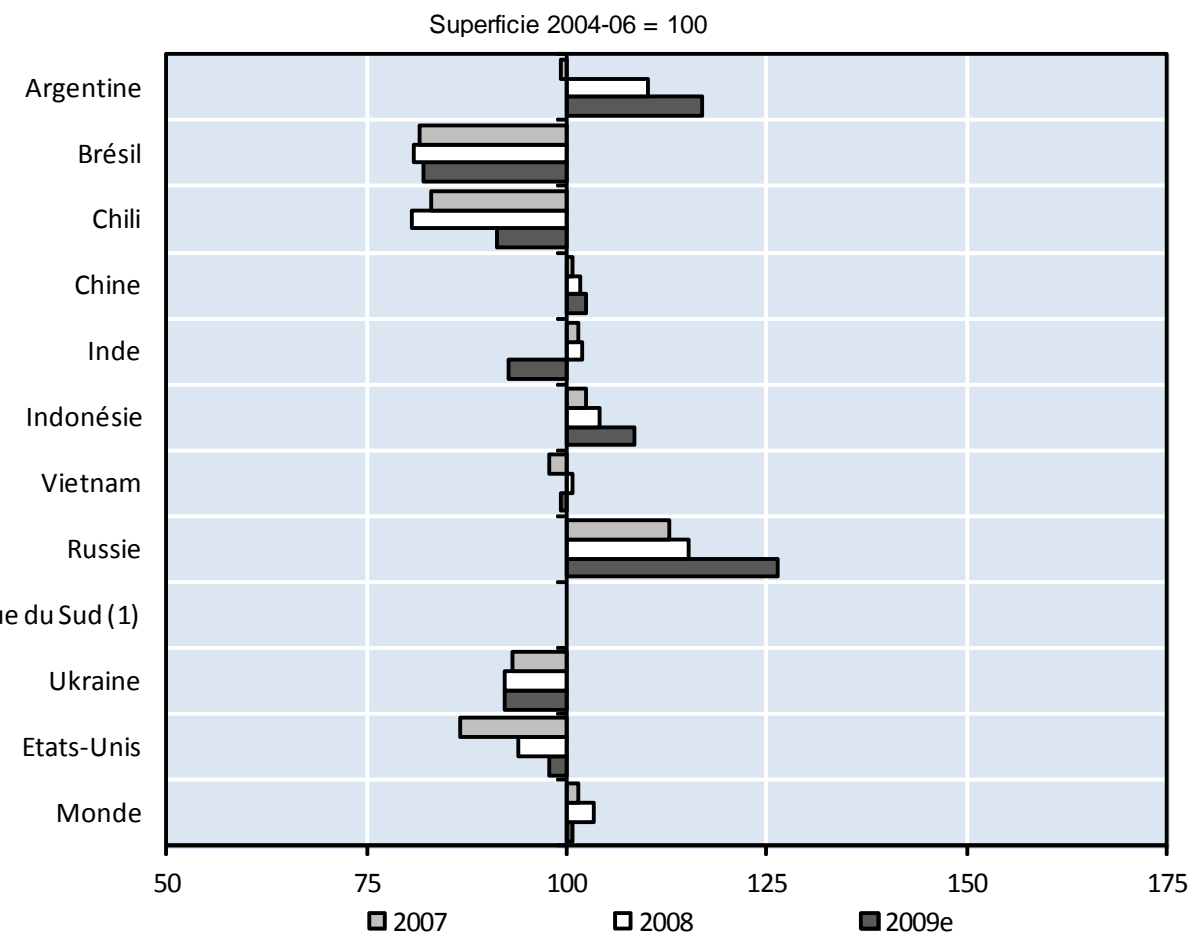

e : estimation.

1. Production commerciale négligeable.

Source : FAOSTAT (2010), Conseil international des céréales (2010) et informations issues de sources nationales et des rapports du Réseau d'information sur l'agriculture mondiale (Global Agriculture Information Network - GAIN) de l'USDA. 
Graphique 2.18. Évolution de la production et des superficies récoltées de soja depuis 2004-06

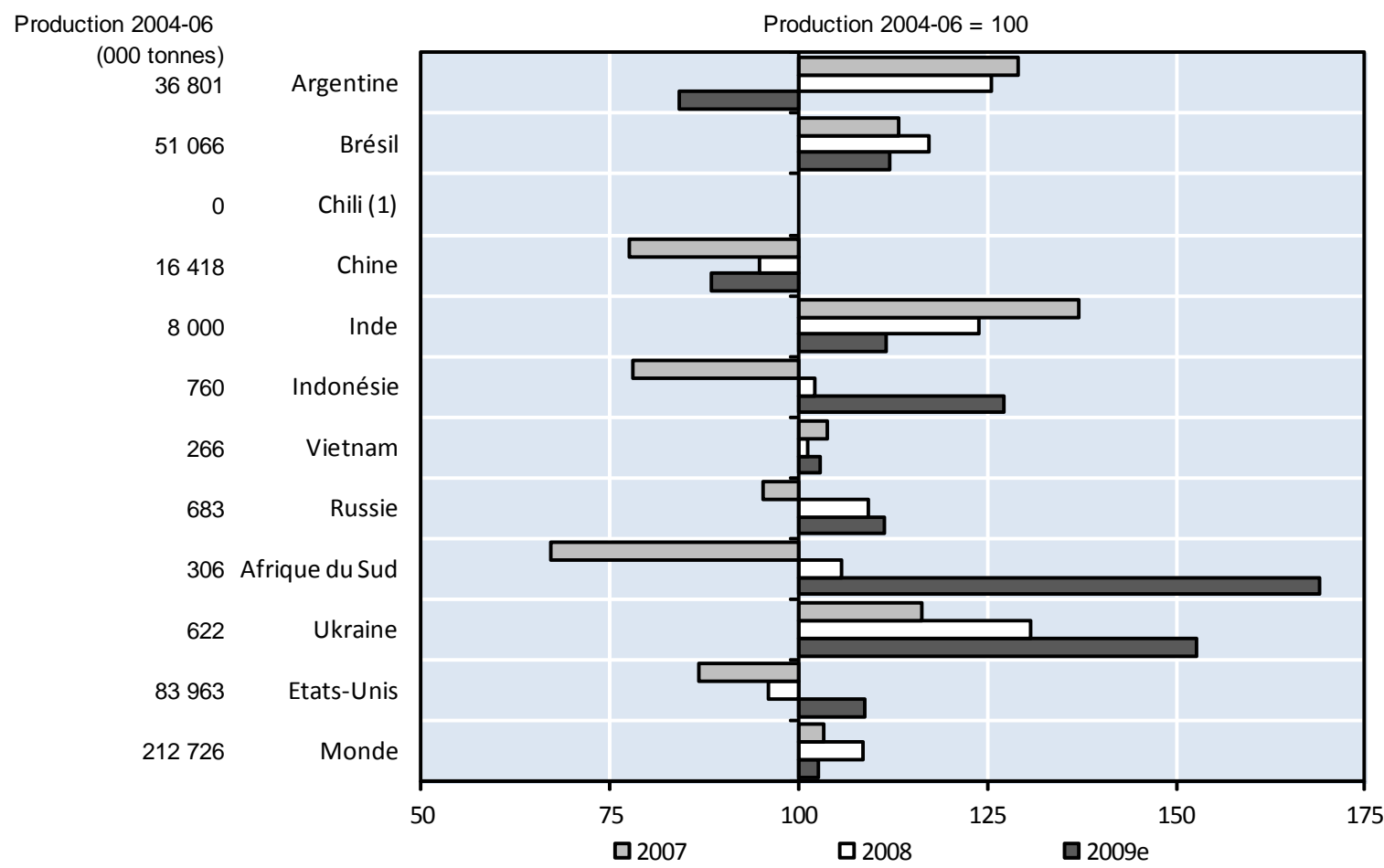

Superficie 2004-06 (000 ha)

14489

22178
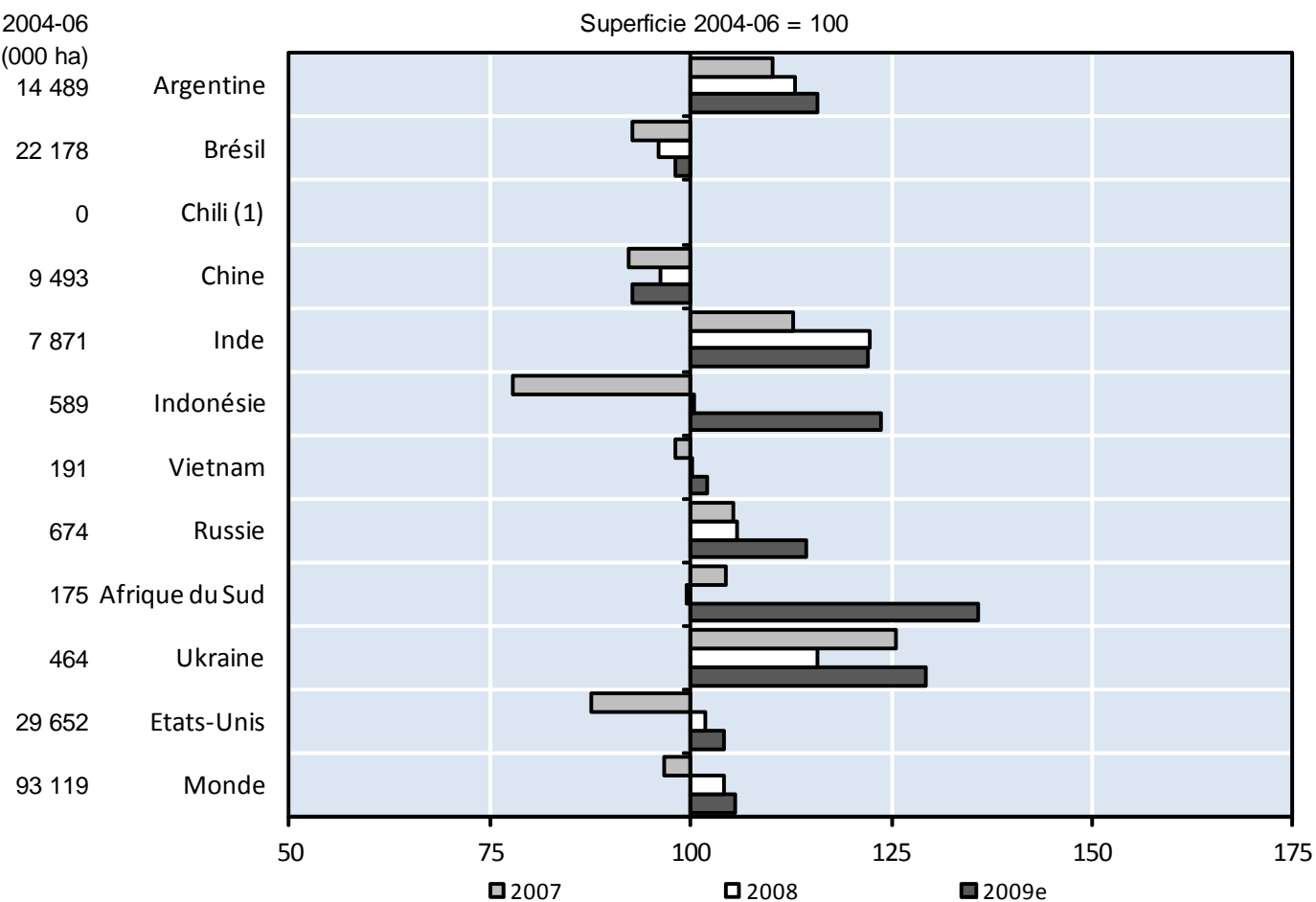

e : estimation.

1. Production commerciale négligeable.

Source : FAOSTAT (2010), Conseil international des céréales (2010) et informations issues de sources nationales et des rapports du Réseau d'information sur l'agriculture mondiale (Global Agriculture Information Network - GAIN) de I'USDA. 


\section{ANNEXE A. TABLEAUX DÉTAILLÉS DES MESURES À COURT TERME PRISES (INCLUANT LE RENFORCEMENT DES MESURES DÉJÀ EN PLACE)}

Annexe tableau A.1. Mesures à court terme prises : Argentine

\begin{tabular}{|c|c|c|c|c|c|c|c|}
\hline \multirow[b]{2}{*}{ Étiquette } & \multirow[b]{2}{*}{ Description de la mesure } & \multirow[b]{2}{*}{ Objectif } & \multirow[b]{2}{*}{ Produit } & \multirow{2}{*}{$\begin{array}{c}\text { Date de } \\
\text { début }\end{array}$} & \multirow[b]{2}{*}{ Date de fin ${ }^{9}$} & \multicolumn{2}{|c|}{$\begin{array}{c}\text { Impact } \\
\text { budgétaire } \\
\text { (millions ARS) }\end{array}$} \\
\hline & & & & & & 2007 & 2008 \\
\hline M2 & $\begin{array}{l}\text { Augmentation des taxes à l'exportation }{ }^{1} \text { de } \\
\text { soja, portées de } 23.5 \% \text { à } 27.5 \% \text {, et de } 20 \% \text { à } \\
24 \% \text { pour les dérivés. }{ }^{2} \text { Le } 12 \text { novembre } 2007 \\
\text { elles ont été de nouveau relevées, à } 35 \% \text { et } \\
32 \% \text { respectivement. }\end{array}$ & $\begin{array}{l}\text { Tirer des recettes } \\
\text { et abriter le } \\
\text { marché de la } \\
\text { hausse des cours } \\
\text { internationaux }\end{array}$ & $\begin{array}{l}\text { Soja et ses } \\
\text { dérivés }\end{array}$ & $\begin{array}{c}15 \text { janv. } \\
2007\end{array}$ & $\begin{array}{c}\text { Toujours en } \\
\text { cours }\end{array}$ & -1238 & -3504 \\
\hline M2 & $\begin{array}{l}\text { Instauration de prix maximums à l'exportation } \\
\text { pour les produits laitiers, les pouvoirs publics } \\
\text { retenant la différence entre le prix à } \\
\text { l'exportation effectif et le prix maximum. À } \\
\text { titre d'exemple, le prix maximum pour la } \\
\text { poudre de lait entier était de } 2470 \text { USD en } \\
2007 \text { et } 3116 \text { USD en } 2008 \text {. }\end{array}$ & $\begin{array}{l}\text { Tirer des recettes } \\
\text { pour } \\
\text { subventionner les } \\
\text { transformateurs } \\
\text { qui limitent les } \\
\text { hausses de prix }\end{array}$ & $\begin{array}{l}\text { Lait et } \\
\text { produits } \\
\text { laitiers }\end{array}$ & Fév. 2007 & Mars 2009 & -194 & -226 \\
\hline M2 & $\begin{array}{l}\text { Augmentation de la taxe à l'exportation de blé, } \\
\text { portée de } 20 \% \text { à } 28 \% \text {. Le } 23 \text { décembre } 2008 \\
\text { elle a été ramenée à } 23 \% \text { et le gouvernement a } \\
\text { annoncé qu'elle serait réduite d'un point de } \\
\text { pourcentage par million de tonnes de } \\
\text { production au-delà du seuil de } 13 \text { millions. }\end{array}$ & $\begin{array}{l}\text { Tirer des recettes } \\
\text { et abriter le } \\
\text { marché de la } \\
\text { hausse des cours } \\
\text { internationaux }\end{array}$ & Blé & 12 nov. 2007 & $\begin{array}{c}\text { Toujours en } \\
\text { cours }\end{array}$ & -135 & -641 \\
\hline M2 & $\begin{array}{l}\text { Augmentation de la taxe à l'exportation de } \\
\text { maïs, portée de } 20 \% \text { à } 25 \% \text {. Le } 23 \text { décembre } \\
\text { elle a été ramenée à } 20 \% \text { et il a été annoncé à } \\
\text { titre d'incitation qu'elle serait réduite d'un } \\
\text { point de pourcentage par million de tonnes de } \\
\text { production au-delà de } 15 \text { millions. }\end{array}$ & $\begin{array}{l}\text { Tirer des recettes } \\
\text { et abriter le } \\
\text { marché de la } \\
\text { hausse des cours } \\
\text { internationaux }\end{array}$ & Maïs & 12 nov. 2007 & $\begin{array}{c}\text { Toujours en } \\
\text { cours }\end{array}$ & -21 & -537 \\
\hline M2 & $\begin{array}{l}\text { Restructuration des taxes à l'exportation, les } \\
\text { droits à taux fixes étant remplacés par des taux } \\
\text { variables établis selon une échelle mobile. S'en } \\
\text { est suivie une nette augmentation des taux de } \\
\text { la taxe à l'exportation pour le soja et le } \\
\text { tournesol, mais une légère réduction pour le } \\
\text { maïs et le blé. Cette nouvelle méthode a } \\
\text { toutefois été rejetée par le Sénat et les taxes à } \\
\text { l'exportation ont retrouvé leur niveau antérieur } \\
\text { à mars } 2008 \text {. }^{3}\end{array}$ & $\begin{array}{l}\text { Enrayer } \\
\text { l'expansion de la } \\
\text { culture du soja au } \\
\text { détriment de la } \\
\text { production } \\
\text { alimentaire } \\
\text { destinée à la } \\
\text { consommation } \\
\text { intérieure }\end{array}$ & $\begin{array}{l}\text { Blé, farine, } \\
\text { maïs, soja } \\
\text { et dérivés }\end{array}$ & $\begin{array}{l}13 \text { mars } \\
2008\end{array}$ & $\begin{array}{l}17 \text { juillet } \\
2008\end{array}$ & -- & -- \\
\hline M2 & $\begin{array}{l}\text { Augmentation de la taxe à l'exportation de } \\
\text { farine de blé, portée de } 10 \% \text { à } 20 \% \text {. Le } 23 \\
\text { décembre } 2008 \text {, elle a été ramenée à } 15 \% \text {. }\end{array}$ & $\begin{array}{l}\text { Réduire les } \\
\text { avantages à } \\
\text { exporter de la } \\
\text { farine de blé } \\
\text { plutôt que du blé }\end{array}$ & $\begin{array}{l}\text { Farine de } \\
\text { blé }\end{array}$ & $\begin{array}{l}28 \text { juillet } \\
2008\end{array}$ & $\begin{array}{c}\text { Toujours en } \\
\text { cours }\end{array}$ & -- & -49 \\
\hline M3 & $\begin{array}{l}\text { Instauration de subventions aux } \\
\text { transformateurs de blé et de maïs qui } \\
\text { approvisionnent le marché national pour } \\
\text { satisfaire la consommation intérieure du pays }{ }^{4}\end{array}$ & $\begin{array}{l}\text { Contrebalancer les } \\
\text { mesures de } \\
\text { contrôle des prix } \\
\text { des produits de } \\
\text { grande } \\
\text { consommation } \\
\text { imposés aux } \\
\text { transformateurs }\end{array}$ & Blé et maïs & Janv. 2007 & $\begin{array}{c}\text { Toujours en } \\
\text { cours }\end{array}$ & 774 & 2190 \\
\hline M3 & $\begin{array}{l}\text { Instauration de subventions aux } \\
\text { transformateurs de lait qui approvisionnent le } \\
\text { marché national pour satisfaire la } \\
\text { consommation intérieure du pays. }\end{array}$ & $\begin{array}{l}\text { Contrebalancer les } \\
\text { mesures de } \\
\text { contrôle des prix } \\
\text { des produits de } \\
\text { grande } \\
\text { consommation } \\
\text { imposés aux } \\
\text { transformateurs }\end{array}$ & $\begin{array}{l}\text { Lait et } \\
\text { produits } \\
\text { laitiers }\end{array}$ & Fév. 2007 & $\begin{array}{c}\text { Toujours en } \\
\text { cours }\end{array}$ & 194 & 226 \\
\hline
\end{tabular}


Annexe tableau A.1. Mesures à court terme prises: Argentine (suite)

\begin{tabular}{|c|c|c|c|c|c|c|c|}
\hline \multirow[b]{2}{*}{ Étiquette } & \multirow[b]{2}{*}{ Description de la mesure } & \multirow[b]{2}{*}{ Objectif } & \multirow[b]{2}{*}{ Produit } & \multirow{2}{*}{$\begin{array}{c}\text { Date de } \\
\text { début }\end{array}$} & \multirow[b]{2}{*}{ Date de fin? } & \multicolumn{2}{|c|}{$\begin{array}{c}\text { Impact } \\
\text { budgétaire } \\
\text { (millions ARS) }\end{array}$} \\
\hline & & & & & & 2007 & 2008 \\
\hline M4 & $\begin{array}{l}\text { Depuis } 2005, \text { les pouvoirs publics ont signé de } \\
\text { nombreux «accords de prix » avec les } \\
\text { transformateurs et/ou les détaillants. Ces } \\
\text { accords sont variables sous l'angle de leur } \\
\text { durée de mise en œuvre, du nombre et du type } \\
\text { de produits couverts, du degré d'augmentation } \\
\text { des prix autorisé, etc. Au cours de la } \\
\text { période 2007-09, les accords de prix avec les } \\
\text { transformateurs de lait, de maïs et de blé ont } \\
\text { été soutenus par des subventions financées } \\
\text { grâce à l'application de taxes à l'exportation et } \\
\text { de prix maximums à l'exportation. }\end{array}$ & $\begin{array}{l}\text { Maîtriser les prix } \\
\text { de détail }\end{array}$ & $\begin{array}{l}\text { Lait, maïs } \\
\text { et blé }\end{array}$ & 2005 & $\begin{array}{l}\text { Toujours en } \\
\text { cours }\end{array}$ & -- & -- \\
\hline M6 & $\begin{array}{l}\text { Clôture de l'enregistrement d'exportations de } \\
\text { maïs lorsque le seuil d'enregistrement de } \\
10.5 \text { million de tonnes }{ }^{5} \text { a été atteint pour la } \\
\text { récolte d'avril-mai } 2007 \text {, encore à venir }\end{array}$ & \multirow{6}{*}{$\begin{array}{l}\text { Éviter les pénuries } \\
\text { intérieures et } \\
\text { maîtriser la hausse } \\
\text { des prix à la } \\
\text { consommation }\end{array}$} & \multirow{6}{*}{ Maïs } & 17 nov. 2006 & 9 mai 2007 & -- & -- \\
\hline M6 & $\begin{array}{l}\text { Réouverture de l'enregistrement des } \\
\text { exportations pour un contingent } \\
\text { supplémentaire de } 3 \text { millions de tonnes de maïs }\end{array}$ & & & 10 mai 2007 & 31 mai 2007 & -- & -- \\
\hline M6 & $\begin{array}{l}\text { Clôture de l'enregistrement des exportations de } \\
\text { maïs }\end{array}$ & & & $1^{\text {er }}$ juin 2007 & $\begin{array}{l}30 \text { janv. } \\
2008\end{array}$ & -- & -- \\
\hline M6 & $\begin{array}{l}\text { Réouverture de l'enregistrement des } \\
\text { exportations de maïs, mais le délai entre } \\
\text { l'enregistrement et l'expédition a été ramené } \\
\text { de un an à } 45 \text { jours. Le } 14 \text { août } 2008 \text {, le délai a } \\
\text { été porté à } 120 \text { jours sous réserve que } \\
\text { l'exportateur acquitte les droits de licence dans } \\
\text { les deux jours de l'obtention de celle-ci. }\end{array}$ & & & $\begin{array}{l}31 \text { janv. } \\
2008\end{array}$ & $\begin{array}{l}16 \text { sept. } \\
2008\end{array}$ & -- & -- \\
\hline M6 & $\begin{array}{l}\text { Clôture de l'enregistrement des exportations de } \\
\text { maïs, bien qu'un contingent supplémentaire de } \\
227500 \text { tonnes de maïs ait été accordé le } \\
17 \text { octobre } 2008\end{array}$ & & & $\begin{array}{l}17 \text { sept. } \\
2008\end{array}$ & $\begin{array}{l}11 \text { janv. } \\
2009\end{array}$ & -- & -- \\
\hline M6 & $\begin{array}{l}\text { Réouverture de l'enregistrement des } \\
\text { exportations de maïs pour un montant total de } \\
3.5 \text { millions de tonnes, un contingent } \\
\text { supplémentaire de } 6 \text { millions de tonnes étant } \\
\text { accordé le } 11 \text { février } 2009\end{array}$ & & & $\begin{array}{c}12 \text { janv. } \\
2009\end{array}$ & $\begin{array}{l}\text { Toujours en } \\
\text { cours }\end{array}$ & -- & -- \\
\hline
\end{tabular}


Annexe tableau A.1. Mesures à court terme prises: Argentine (suite)

\begin{tabular}{|c|c|c|c|c|c|c|c|}
\hline \multirow[b]{2}{*}{ Étiquette } & \multirow[b]{2}{*}{ Description de la mesure } & \multirow[b]{2}{*}{ Objectif } & \multirow[b]{2}{*}{ Produit } & \multirow{2}{*}{$\begin{array}{c}\text { Date de } \\
\text { début }\end{array}$} & \multirow[b]{2}{*}{ Date de fin' } & \multicolumn{2}{|c|}{$\begin{array}{c}\text { Impact } \\
\text { budgétaire } \\
\text { (millions ARS) }\end{array}$} \\
\hline & & & & & & 2007 & 2008 \\
\hline M6 & $\begin{array}{l}\text { Clôture de l'enregistrement des exportations de } \\
\text { maïs }{ }^{6}\end{array}$ & \multirow{5}{*}{$\begin{array}{l}\text { Éviter les pénuries } \\
\text { intérieures et } \\
\text { maîtriser la hausse } \\
\text { des prix à la } \\
\text { consommation }\end{array}$} & \multirow{5}{*}{ Blé } & 8 mars 2007 & 13 nov. 2007 & -- & -- \\
\hline M6 & $\begin{array}{l}\text { Réouverture de l'enregistrement des } \\
\text { exportations de blé, mais le délai entre } \\
\text { l'enregistrement et l'expédition a été ramené } \\
\text { de un an à } 90 \text { jours. Pendant les } 13 \text { jours } \\
\text { d'ouverture de l'enregistrement des } \\
\text { exportations de blé, plus de } 7 \text { millions de } \\
\text { tonnes en ont bénéficié. }\end{array}$ & & & 14 nov. 2007 & 27 nov. 2007 & -- & -- \\
\hline M6 & $\begin{array}{l}\text { Clôture de l'enregistrement des exportations de } \\
\text { blé à la suite de fortes gelées dans les grandes } \\
\text { régions céréalières. }\end{array}$ & & & 28 nov. 2007 & 21 mai 2008 & -- & -- \\
\hline M6 & $\begin{array}{l}\text { Réouverture de l'enregistrement des } \\
\text { exportations de blé, mais le délai entre } \\
\text { l'enregistrement et l'expédition a été ramené } \\
\text { de } 90 \text { à } 30 \text { jours. }{ }^{7} \text { L'enregistrement était } \\
\text { initialement limité à } 100000 \text { tonnes destinées à } \\
\text { être exportées vers le Brésil. Ouverture, le } \\
11 \text { juin } 2008 \text {, de l'enregistrement pour } \\
1 \text { million de tonnes - la moitié pour le Brésil et } \\
\text { la moitié pour les autres marchés traditionnels, } \\
\text { tels que la Bolivie. Ouverture de } \\
\text { l'enregistrement pour un contingent } \\
\text { supplémentaire de } 0.6 \text { million de tonnes le } \\
31 \text { juillet, de } 1.44 \text { million de tonnes le } 21 \text { août, } \\
\text { de } 1.5 \text { million de tonnes le } 3 \text { octobre et de } \\
1 \text { million le } 24 \text { novembre. Le } 14 \text { août } 2008 \text {, le } \\
\text { délai a été porté à } 90 \text { jours sous réserve que } \\
\text { l'exportateur acquitte les droits de licence dans } \\
\text { les deux jours de l'obtention de celle-ci. }\end{array}$ & & & 22 mai 2008 & Nov. 2008 & -- & -- \\
\hline M6 & $\begin{array}{l}\text { Réouverture de l'enregistrement des } \\
\text { exportations de blé dans une limite initialement } \\
\text { fixée à } 1 \text { million de tonnes. Des contingents } \\
\text { supplémentaires ont porté le total à } \\
7.4 \text { millions de tonnes. }\end{array}$ & & & 6 déc. 2008 & $\begin{array}{c}\text { Toujours en } \\
\text { cours }\end{array}$ & -- & -- \\
\hline M6 & $\begin{array}{l}\text { Clôture de l'enregistrement des exportations de } \\
\text { soja et de ses dérivés. }\end{array}$ & \multirow[b]{2}{*}{$\begin{array}{l}\text { Éviter les pénuries } \\
\text { intérieures et } \\
\text { maîtriser la hausse } \\
\text { des prix à la } \\
\text { consommation }\end{array}$} & \multirow[b]{2}{*}{$\begin{array}{l}\text { Soja et ses } \\
\text { dérivés }\end{array}$} & 8 nov. 2007 & 13 nov. 2007 & -- & -- \\
\hline M6 & $\begin{array}{l}\text { Réouverture de l'enregistrement des } \\
\text { exportations mais le délai entre } \\
\text { l'enregistrement et l'expédition a été ramené } \\
\text { de un an à } 150 \text { jours. Le } 14 \text { août } 2008 \text {, le délai } \\
\text { a été porté à } 180 \text { jours sous réserve que } \\
\text { l'exportateur acquitte les droits de licence dans } \\
\text { les deux jours de l'obtention de celle-ci. }\end{array}$ & & & 14 nov. 2007 & $\begin{array}{c}\text { Toujours en } \\
\text { cours }\end{array}$ & -- & -- \\
\hline I1 & $\begin{array}{l}\text { La Banque centrale argentine a développé ses } \\
\text { interventions sur le marché en accroissant ses } \\
\text { émissions de titres et de bons. }\end{array}$ & $\begin{array}{l}\text { Réduire } \\
\text { l'augmentation de } \\
\text { l'offre de monnaie } \\
\text { générée par } \\
\text { l'achat de devises } \\
\text { afin de maintenir } \\
\text { la stabilité de la } \\
\text { monnaie }\end{array}$ & Tous & $\begin{array}{l}\text { Précé- } \\
\text { demment }\end{array}$ & T3 2008 & -- & -- \\
\hline I1 & $\begin{array}{l}\text { La Banque centrale argentine a régulièrement } \\
\text { relevé ses taux débiteur (prise en pension de } \\
\text { titres) et créditeur (mise en pension de titres) } \\
\text { depuis le début de ses interventions à la mi- } \\
2004 \text {. }\end{array}$ & $\begin{array}{l}\text { Freiner la } \\
\text { demande et } \\
\text { l'inflation }\end{array}$ & Tous & mi-2004 & $\begin{array}{c}7 \text { juillet } \\
2009\end{array}$ & -- & -- \\
\hline $\mathrm{P} 2$ & Subventions aux producteurs de blé et de maïs ${ }^{4}$ & $\begin{array}{l}\text { Dédommager les } \\
\text { producteurs de } \\
\text { l'instauration de } \\
\text { prix fixes pour les } \\
\text { ventes aux } \\
\text { transformateurs }^{8}\end{array}$ & Blé et maïs & Janv. 2007 & $\begin{array}{c}\text { Toujours en } \\
\text { cours }\end{array}$ & 774 & 2190 \\
\hline
\end{tabular}


«-- » : aucun impact budgétaire ; « n.d. » : non disponible.

1. Des droits à l'exportation de $10 \%$ ou $5 \%$ ont été instaurés en mars 2002 sur toutes les marchandises en vue d'amortir les répercussions sur les prix intérieurs de la forte dévaluation nominale du peso (supérieure à 200\%) et de compenser l'effondrement des recettes fiscales. La plupart des recettes tirées de la taxe à l'exportation ont été consacrés au financement du programme Jefes y Jefas de hogar, consistant en transferts conditionnels en espèces au profit des pauvres. Depuis sa mise en place, les décisions successivement prises ont modifié les taux de cette taxe sur les exportations, le montant des droits applicables allant de $5 \%$ à $45 \%$ de leur valeur f.à.b.

2. Les recettes tirées de cette augmentation servent au financement des programmes de compensation lancés en 2007.

3. Les agriculteurs ont énergiquement protesté contre l'instauration de ce système de taxes à l'exportation de montant variable, retardant la récolte et la vente du produit, bloquant les routes et manifestant dans les rues.

4. Il a été estimé que l'augmentation de la taxe à l'exportation sur le soja et ses dérivés en janvier 2007 fournissait $80 \%$ des recettes nécessaires pour couvrir le coût des subventions accordées à l'industrie et aux producteurs nationaux, imposant au gouvernement de trouver d'autres sources de financement pour le complément. On ignore comment la subvention est répartie entre les transformateurs et les producteurs de blé et de maïs mais on part de l'hypothèse qu'ils se la partagent en deux moitiés égales.

5. Depuis 1992, l'Argentine a appliqué un système d'enregistrement préalable de l'ensemble des exportations de marchandises. Les pouvoirs publics contrôlent les exportations de céréales et d'oléagineux en suspendant provisoirement l'enregistrement des ventes à l'exportation et en modifiant le délai autorisé entre l'enregistrement et l'expédition des exportations.

6. L'enregistrement des exportations de farine de blé a également été clos le 8 mars 2007, mais il a été rouvert le 14 mars 2007.

7. Le gouvernement a annoncé à plusieurs reprises au cours du premier semestre 2008 que l'enregistrement des exportations reprendrait bientôt, mais ce n'était que pour le remettre à plus tard

8. Le prix fixe pour le blé était ainsi de 370 ARS/tonne (120 USD/tonne) en 2007 et 2008, avant d'être porté à 420 ARS/tonne (113 USD/tonne) en 2009

9. La mention « Toujours en cours » indique que la mesure considérée était encore en vigueur au 31 décembre 2009.

Sources:

Banque centrale argentine (2009), Inflation Report, diverses années, www.bcra.gov.ar.

GAIN-AR7008 (2007), Argentina: Agricultural Situation - Argentina imposes variable export taxes; raises duty on major commodities, USDA/FAS, 3 avril.

GAIN-AR8016 (2008), Argentina: Grain and Feed - Annual, USDA/FAS, 12 avril.

GAIN-AR8021 (2008), Argentina: Oilseeds - Annual, USDA/FAS, 9 mai.

GAIN-AR9005 (2009), Argentina: Grain and Feed - Annual, USDA/FAS, 13 mars.

GAIN-AR9013 (2009), Argentina: Oilseeds - Annual, USDA/FAS, 16 avril.

Banque mondiale (2006), Argentina Agriculture and Rural Development: Selected Issues, rapport $n^{\circ} 32763-A R$, Banque mondiale, Washington DC, www-

wds.worldbank.org/external/default/WDSContentServer/WDSP/IB/2006/10/18/000090341 20061018084304/Rendered/PDF/32763.p df.

OMC (2007), Examen des politiques commerciales : Argentine, WT/TPR/S/176, OMC, Genève, 8 janvier,

http://www.wto.org/french/tratop f/tpr f/tp277 f.htm. 
Annexe tableau A.2. Mesures à court terme prises : Brésil

\begin{tabular}{|c|c|c|c|c|c|c|c|}
\hline \multirow[b]{2}{*}{ Étiquette } & \multirow[b]{2}{*}{ Description de la mesure } & \multirow[b]{2}{*}{ Objectif } & \multirow[b]{2}{*}{ Produit } & \multirow{2}{*}{$\begin{array}{l}\text { Date de } \\
\text { début }\end{array}$} & \multirow{2}{*}{$\begin{array}{l}\text { Date de } \\
\text { fin }^{6}\end{array}$} & \multicolumn{2}{|c|}{$\begin{array}{c}\text { Impact budgétaire } \\
\text { (millions BRL) }\end{array}$} \\
\hline & & & & & & 2007 & 2008 \\
\hline M1 & $\begin{array}{l}\text { Instauration d'un contingent tarifaire } \\
\text { exempt de droits pour } 1 \text { million de } \\
\text { tonnes de blé en provenance de pays } \\
\text { extérieurs au Mercosur, dont } \\
\text { l'importation devait intervenir avant la } \\
\text { fin juin } 2008 \text {. En mai, le volume en a été } \\
\text { porté à } 2 \text { millions de tonnes et le délai } \\
\text { d'importation a été prorogé jusqu'au } \\
31 \text { août. Le tarif extérieur } \\
\text { commun (TEC) est de } 10 \% \text {, et les } \\
\text { importations originaires des pays du } \\
\text { Mercosur sont déjà admises en } \\
\text { franchise. }\end{array}$ & $\begin{array}{l}\text { Éviter les pénuries } \\
\text { intérieures compte } \\
\text { tenu des quantités } \\
\text { limitées de produits } \\
\text { qui pouvaient être } \\
\text { importées } \\
\text { d'Argentine. }\end{array}$ & Blé & 6 fév. 2008 & $\begin{array}{l}31 \text { août } \\
2008\end{array}$ & -- & 78 \\
\hline M1 & $\begin{array}{l}\text { Instauration d'un contingent tarifaire de } \\
80000 \text { tonnes de sardines bénéficiant } \\
\text { d'un taux de } 2 \% \text {. Le TEC est de } 10 \% \text {. }\end{array}$ & $\begin{array}{l}\text { Éviter les pénuries } \\
\text { intérieures }\end{array}$ & Sardines & 16 avr. 2008 & $\begin{array}{c}15 \text { avr. } \\
2009\end{array}$ & -- & 4 \\
\hline M1 & $\begin{array}{l}\text { Suppression du prélèvement applicable } \\
\text { à la marine marchande (AFRMM) } \\
\text { correspondant à } 25 \% \text { de la valeur des } \\
\text { cargaisons transitant par les ports } \\
\text { brésiliens }\end{array}$ & $\begin{array}{l}\text { Réduire les prix aux } \\
\text { frontières }\end{array}$ & $\begin{array}{c}\text { Blé et farine } \\
\text { de blé }\end{array}$ & Mai 2008 & Déc. 2008 & -- & 14 \\
\hline M1 & $\begin{array}{l}\text { Instauration d'un contingent tarifaire de } \\
72500 \text { tonnes d'huile de palmiste } \\
\text { bénéficiant d'un taux de } 2 \% \text {. Le TEC est } \\
\text { de } 10 \% \text {. }\end{array}$ & $\begin{array}{l}\text { Éviter les pénuries } \\
\text { intérieures }\end{array}$ & $\begin{array}{c}\text { Huile de } \\
\text { palme brute }\end{array}$ & $1^{\mathrm{er}}$ août 2008 & $\begin{array}{l}31 \text { juil. } \\
2009\end{array}$ & -- & 1 \\
\hline M3 & $\begin{array}{l}\text { Suppression de la contribution sociale } \\
\text { de } 9.25 \% \text { appliquée dans le cadre du } \\
\text { programme PIS/Confins }\end{array}$ & $\begin{array}{l}\text { Réduire les prix à la } \\
\text { consommation }\end{array}$ & $\begin{array}{l}\text { Divers } \\
\text { produits }^{1}\end{array}$ & Juin 2007 & $\begin{array}{l}\text { Toujours } \\
\text { en cours }\end{array}$ & n.d. & n.d. \\
\hline M3 & $\begin{array}{l}\text { Suppression de la contribution sociale } \\
\text { de } 9.25 \% \text { appliquée dans le cadre du } \\
\text { programme PIS/Confins }\end{array}$ & $\begin{array}{l}\text { Réduire les prix à la } \\
\text { consommation }\end{array}$ & $\begin{array}{l}\text { Blé, farine de } \\
\text { blé et pain }\end{array}$ & Mai 2008 & Déc. 2008 & -- & 500 \\
\hline M7 & Déblocage de stocks publics & $\begin{array}{l}\text { Maîtriser l'ampleur } \\
\text { de la hausse des prix }\end{array}$ & $\begin{array}{l}\text { Riz, maïs, blé } \\
\text { et haricots }\end{array}$ & 2006 & 2008 & -- & -- \\
\hline I1 & $\begin{array}{l}\text { La Banque centrale du Brésil a relevé de } \\
250 \text { points de base son taux de politique } \\
\text { monétaire (Selic), passé de } 11.25 \% \text { à } \\
13.75 \% \text {, d'avril } 2008 \text { à septembre } 2008^{2}\end{array}$ & $\begin{array}{l}\text { Freiner la demande } \\
\text { et l'inflation }\end{array}$ & Tous & Avril 2008 & Janv. 2009 & -- & -- \\
\hline $\mathrm{C} 1$ & $\begin{array}{l}\text { Augmentation des niveaux de prestation } \\
\text { fournis par Bolsa Família, de } 19 \% \text { en } \\
2007 \text { puis de } 15 \% \text { supplémentaires en } \\
2008^{3}\end{array}$ & $\begin{array}{l}\text { Aider les personnes } \\
\text { touchées par les } \\
\text { hausses de prix }\end{array}$ & Tous & 2007 & 2008 & 1441 & 2843 \\
\hline $\mathrm{P} 1$ & $\begin{array}{l}\text { Augmentation des prix minimums } \\
\text { garantis pouvant atteindre jusqu'à } 20 \% \\
\text { pour les achats publics directs dans le } \\
\text { cadre des programmes AGF et Option } \\
\text { publique en 2008/09 }\end{array}$ & $\begin{array}{l}\text { Accroître le niveau } \\
\text { des stocks publics }\end{array}$ & $\begin{array}{l}\text { Un large } \\
\text { éventail }\end{array}$ & Juin 2008 & $\begin{array}{l}\text { Toujours } \\
\text { en cours }\end{array}$ & -- & 54 \\
\hline $\mathrm{P} 2$ & $\begin{array}{l}\text { Suppression des droits à l'importation } \\
\text { de } 4 \% \text { à } 6 \%\end{array}$ & $\begin{array}{l}\text { Réduire les coûts de } \\
\text { production }\end{array}$ & $\begin{array}{l}\text { Certains } \\
\text { engrais } \\
\text { minéraux et } \\
\text { chimiques } \\
\end{array}$ & Sept. 2007 & $\begin{array}{l}\text { Toujours } \\
\text { en cours }\end{array}$ & -- & 28 \\
\hline $\mathrm{P} 2$ & $\begin{array}{l}\text { Lancement du Plan national en faveur } \\
\text { du blé, qui offre une ligne de crédit } \\
\text { supplémentaire de } 1.2 \text { milliard BRL au } \\
\text { taux préférentiel de } 6.75 \%\end{array}$ & $\begin{array}{l}\text { Accroître la } \\
\text { production de } 25 \%\end{array}$ & Blé & Avril 2008 & $\begin{array}{l}\text { Toujours } \\
\text { en cours }\end{array}$ & -- & 69 \\
\hline
\end{tabular}


Annexe tableau A.2. Mesures à court terme prises : Brésil (suite)

\begin{tabular}{|c|c|c|c|c|c|c|c|}
\hline \multirow[b]{2}{*}{ Étiquette } & \multirow[b]{2}{*}{ Description de la mesure } & \multirow[b]{2}{*}{ Objectif } & \multirow[b]{2}{*}{ Produit } & \multirow{2}{*}{$\begin{array}{c}\text { Date de } \\
\text { début }\end{array}$} & \multirow[b]{2}{*}{ Date de fin ${ }^{6}$} & \multicolumn{2}{|c|}{$\begin{array}{c}\text { Impact budgétaire } \\
\text { (millions BRL) }\end{array}$} \\
\hline & & & & & & 2007 & 2008 \\
\hline $\mathrm{P} 2$ et $\mathrm{P} 3$ & $\begin{array}{l}\text { Accroître le montant des crédits bonifiés } \\
\text { dont peuvent bénéficier les producteurs } \\
\text { agricoles commerciaux (moyenne à } \\
\text { grande échelle), portés de } 7 \text { milliards } \\
\text { BRL (12\%) à } 65 \text { milliards BRL pour la } \\
\text { campagne agricole } 2008 / 09 \text {. Des } \\
\text { ressources supplémentaires étaient mises } \\
\text { à leur disposition pour le financement } \\
\text { du fonds de roulement (P2) et des } \\
\text { investissements (P3). }\end{array}$ & $\begin{array}{l}\text { Accroître la } \\
\text { production afin de } \\
\text { porter le niveau des } \\
\text { stocks publics de } \\
1.5 \text { million de } \\
\text { tonnes en } 2008 \text { à } \\
6 \text { millions de tonnes } \\
\text { en } 2009\end{array}$ & Tous & Juil. 2008 & $\begin{array}{c}\text { Toujours en } \\
\text { cours }\end{array}$ & -- & 193 \\
\hline $\mathrm{P} 3$ & $\begin{array}{l}\text { Lancement du programme } \text { «Plus de } \\
\text { nourriture », qui fournit une nouvelle } \\
\text { ligne de crédit de } 6 \text { milliards BRL aux } \\
\text { exploitants pratiquant une agriculture } \\
\text { familiale couverts par le PRONAF. Il } \\
\text { comporte également un accord avec les } \\
\text { constructeurs visant à réduire de } 11 \% \text { - } \\
15 \% \text { le prix d'achat des machines. }\end{array}$ & $\begin{array}{l}\text { Aider les exploitants } \\
\text { pratiquant une } \\
\text { agriculture familiale } \\
\text { à acheter jusqu'à } \\
60000 \text { tracteurs et } \\
300000 \text { machines et } \\
\text { accessoires agricoles } \\
\text { avant } 2010\end{array}$ & $\begin{array}{l}\text { Un large } \\
\text { éventail de } \\
\text { produits }{ }^{5}\end{array}$ & Juil. 2008 & $\begin{array}{c}\text { Toujours en } \\
\text { cours }\end{array}$ & -- & 300 \\
\hline $\mathrm{P} 4$ & $\begin{array}{l}\text { Le programme «Plus de nourriture » } \\
\text { prévoyait en outre un renforcement de } \\
\text { l'assistance technique et des services de } \\
\text { vulgarisation rurale }\end{array}$ & $\begin{array}{l}\text { Accroître la } \\
\text { disponibilité de } \\
\text { technologies } \\
\text { appropriées et aider } \\
\text { à l'organisation des } \\
\text { exploitations } \\
\text { familiales. }\end{array}$ & $\begin{array}{l}\text { Un large } \\
\text { éventail de } \\
\text { produits }^{5}\end{array}$ & Juil. 2008 & $\begin{array}{c}\text { Toujours en } \\
\text { cours }\end{array}$ & -- & 229 \\
\hline
\end{tabular}

«-- » : aucun impact budgétaire ; « n.d. » : non disponible.

1. Y compris le lait écrémé en poudre, les boissons fermentées, les préparations destinées aux nourrissons, certains fromages et le lactosérum destiné à la consommation humaine.

2. La date de fin correspond au mois où le taux d'intérêt de la politique monétaire a été réduit afin de stimuler l'économie.

3. Bolsa Família ( « prime familiale ») est issu de la fusion, en octobre 2003, de quatre programmes de transfert préexistants. C'est un programme de transferts conditionnels en espèces au profit des familles pauvres dont les revenus sont inférieurs au seuil minimum. Le montant nominal en a été maintenu constant depuis 2003 jusqu'en juillet 2007, malgré un renchérissement de $16.7 \%$ du coût de la vie. En juillet 2007, le Décret 6.157 a relevé de $17 \%$ à $20 \%$ le montant des transferts (selon les catégories), ce qui leur a permis de retrouver leur niveau réel initial (Grosh et al., 2009). Il a de nouveau été accru en 2008. Le montant des transferts a en outre été encore une fois majoré en 2009 , mais c'était en l'occurrence pour cause de ralentissement économique. Près de $90 \%$ en sont utilisés pour l'achat de nourriture (Curralero, 2009).

4. Les exploitants agricoles ont pu emprunter jusqu'à $100000 \mathrm{BRL}$ à un taux de $2 \%$ pour l'achat de tracteurs et d'autres machines et accessoires agricoles. L'objectif global est d'accroître de 18 millions de tonnes la production de ce secteur à l'horizon 2010 , soit une augmentation de $17 \%$.

5. Maïs, manioc, lait, soja, fruits, riz, volaille, viande de porc, blé, haricots, café et oignons.

6. La mention « Toujours en cours » indique que la mesure considérée était encore en vigueur au 31 décembre 2009.

Sources:

Banque centrale du Brésil (2009), Inflation Report, diverses années, www.bcb.gov.br.

Curralero, Claudia (2009), "Bolsa Família Program", communication présentée au Forum de la Banque mondiale sur l'apprentissage Sud-Sud : South-South Learning Forum - Social Protection Responses to the Three Waves of Crisis: Finance, Food \& Fuel, Le Caire, juin 15-18, http://info.worldbank.org/etools/docs/library/251776/D2.1.2 Claudia\%20Curralero BOLSABrazil 6-16.pdf.

França, Caio Galvão de (2009), "Brazil: Short-term response to higher food prices", communication présentée au Forum mondial sur l'agriculture de l'OCDE de 2009, www.oecd.org/agriculture/globalforum/iune09.

GAIN-BR8603 (2008), Brazil: Grain and Feed - Brazil temporarily lowers wheat import tariff, USDA/FAS, 8 février.

GAIN-BR8610 (2008), Brazil: Grain and Feed - Annual Report 2008, USDA/FAS, 19 mars.

GAIN-BR8612 (2008), Brazil: Oilseeds and Products - Annual Soybean Report 2008, USDA/FAS, 27 mai.

GAIN-BR9611 (2009), Brazil: Grain and Feed - Annual Report 2009, USDA/FAS, 18 mars.

GAIN-BR9613 (2009), Brazil: Oilseeds - Annual Report 2009, USDA/FAS, 15 avril.

Grosh, M., et al. (2008), Pour la protection et la promotion: Conception et mise en œuvre de filets sociaux de sécurité efficaces,

Banque mondiale, Washington, DC,

http://web.worldbank.org/WBSITE/EXTERNAL/TOPICS/EXTSOCIALPROTECTION/EXTSAFETYNETSANDTRANSFERS/O, content

MDK:21949854 pagePK:148956 piPK:216618 theSitePK:282761,00.html.

Marques, Vicente Azevedo (2008), "Report on main policy development in Brazil", rapport soumis à l'OCDE. 
Annexe tableau A.3. Mesures à court terme prises : Chili

\begin{tabular}{|c|c|c|c|c|c|c|c|}
\hline \multirow[b]{2}{*}{ Étiquette } & \multirow[b]{2}{*}{ Description de la mesure } & \multirow[b]{2}{*}{ Objectif } & \multirow[b]{2}{*}{ Produit } & \multirow{2}{*}{$\begin{array}{c}\text { Date de } \\
\text { début }\end{array}$} & \multirow[b]{2}{*}{ Date de fin ${ }^{3}$} & \multicolumn{2}{|c|}{$\begin{array}{c}\text { Impact } \\
\text { budgétaire } \\
\text { (millions CHL) }\end{array}$} \\
\hline & & & & & & 2007 & 2008 \\
\hline M9 & $\begin{array}{l}\text { Le ministère de l'Agriculture publie au travers } \\
\text { de l'Office des politiques et études } \\
\text { agricoles (OPEDA) des informations sur les } \\
\text { prix à la consommation dans les secteurs } \\
\text { stratégiques et sur les marchés de Santiago. } \\
\text { Cela a commencé par les fruits et légumes en } \\
\text { mars 2008. Les prix de la viande rouge y ont } \\
\text { été ajoutés en octobre et ceux du pain en } \\
\text { novembre. Les prix des produits laitiers sont } \\
\text { communiqués depuis } 2009 \text {. }\end{array}$ & $\begin{array}{l}\text { Améliorer la } \\
\text { chaîne de la } \\
\text { valeur } \\
\text { ajoutée et } \\
\text { éviter les } \\
\text { comportemen } \\
\text { ts spéculatifs }\end{array}$ & Tous & Mars 2008 & $\begin{array}{c}\text { Toujours en } \\
\text { cours }\end{array}$ & -- & n.d. \\
\hline I1 & $\begin{array}{l}\text { La Banque centrale du Chili a relevé de } 325 \\
\text { points de base son taux de politique monétaire, } \\
\text { passé de } 5 \% \text { à } 8.25 \% \text {, de juillet } 2007 \text { à } \\
\text { septembre } 2008\end{array}$ & $\begin{array}{l}\text { Freiner la } \\
\text { demande et } \\
\text { l'inflation }\end{array}$ & Tous & Juil. 2007 & Janv. $2009^{1}$ & -- & -- \\
\hline $\mathrm{C} 1$ & $\begin{array}{l}\text { Prime exceptionnelle de } 20000 \text { CLP ( } 35 \text { USD) } \\
\text { au profit de } 1.4 \text { million de ménages } \\
\text { bénéficiaires de trois programmes d'aide } \\
\text { sociale. }{ }^{2} \mathrm{Au} \text { total, } 5.6 \text { millions de personnes, } \\
\text { soit les } 40 \% \text { les plus pauvres de la population, } \\
\text { ont bénéficié de paiements en espèces. }\end{array}$ & $\begin{array}{l}\text { Aider les } \\
\text { personnes les } \\
\text { plus touchées } \\
\text { par la hausse } \\
\text { des prix de } \\
\text { l'énergie et } \\
\text { des produits } \\
\text { alimentaires }\end{array}$ & Tous & Mai 2008 & Mai 2008 & -- & 28810 \\
\hline
\end{tabular}

«-- » : aucun impact budgétaire ; « n.d. » : non disponible.

1. La date de fin correspond au mois où le taux d'intérêt de la politique monétaire a été réduit afin de stimuler l'économie.

2. Les bénéficiaires en ont été : les 300000 ménages affiliés à Chile Solidario, qui aide les $5 \%$ les plus pauvres de la population à lutter contre divers obstacles à l'intégration sociale ; les 515000 familles bénéficiaires du Subsidio Unitario Familiar, " allocation familiale unifiée » versée aux pauvres au travers de l'Institut de normalisation prévisionnelle (Instituto de Normalización Previsional INP) ; et les 600000 travailleurs qui perçoivent des allocations familiales et dont le salaire annuel était en mars 2008 inférieur à $250000 \mathrm{CHL}$.

3. La mention « Toujours en cours » indique que la mesure considérée était encore en vigueur au 31 décembre 2009.

Sources:

Banque centrale du Chili (2009), Monetary Policy Report, divers numéros, www.bcentral.cl/eng/publications/policies/polit02.htm. Commission économique pour l'Amérique latine et les Caraïbes [CEPALC] (2008), Economic Survey of Latin America and the Caribbean 2007-08, CEPALC, www.cepal.org/cgi-bin/getProd.asp?xml=/publicaciones/xml/3/33873/P33873.xm/\&xsl=/de/tpli/p9f.xsl\&base=/tpl-i/top-bottom.xslt.

De Gregorio, José (2009), Rise and fall in commodity prices: Economic impact and policy responses, Gouverneur, Banque centrale du Chili, www.bcentral.cl/politicas/presentaciones/consejeros/pdf/2009/jdg23032009.pdf.

Ministère des Finances (2008), Communiqué de presse,

www. minhda.cl/english/prensa/detalle.php?id=12663\&code=c/UkV3EwupA/2).

García Silva, Pablo (2008), The global rise in food prices and the US slowdown: Issues and challenges in monetary policy - the case of Chile, Directeur de la recherche, Banque centrale du Chili,

http://siteresources.worldbank.org/EXTLACOFFICEOFCE/Resources/870892-1214424140903/Garcia.pdf. 
Annexe tableau A.4. Mesures à court terme prises : Chine

\begin{tabular}{|c|c|c|c|c|c|c|c|}
\hline \multirow[b]{2}{*}{ Étiquette } & \multirow[b]{2}{*}{ Description de la mesure } & \multirow[b]{2}{*}{ Objectif } & \multirow[b]{2}{*}{ Produit } & \multirow{2}{*}{$\begin{array}{c}\text { Date de } \\
\text { début }\end{array}$} & \multirow[b]{2}{*}{ Date de fin' } & \multicolumn{2}{|c|}{$\begin{array}{c}\text { Impact } \\
\text { budgétaire } \\
\text { (millions CNY) }\end{array}$} \\
\hline & & & & & & 2007 & 2008 \\
\hline M1 & $\begin{array}{l}\text { Réduction des droits de douane à } \\
\text { l'importation, ramenés de } 3 \% \text { à } 1 \%\end{array}$ & $\begin{array}{l}\text { Stabiliser les prix } \\
\text { intérieurs des } \\
\text { oléagineux }\end{array}$ & Soja & $1^{\mathrm{er}}$ oct. 2007 & $\begin{array}{l}30 \text { sept. } \\
2008\end{array}$ & 436 & 2274 \\
\hline M1 & $\begin{array}{l}\text { Réduction des droits de douane à } \\
\text { l'importation, ramenés de } 10 \% \text { à } \\
5 \%\end{array}$ & $\begin{array}{l}\text { Accroître l'offre pour } \\
\text { répondre à la demande } \\
\text { croissante des } \\
\text { consommateurs }\end{array}$ & $\begin{array}{l}\text { Huile d'olive } \\
\text { et huile de } \\
\text { coco }\end{array}$ & $1^{\mathrm{er}}$ juin 2008 & $\begin{array}{l}30 \text { sept. } \\
2008\end{array}$ & -- & 20 \\
\hline M1 & $\begin{array}{l}\text { Réduction des droits de douane à } \\
\text { l'importation, ramenés de } 6 \%-25 \% \\
\text { à } 5 \%-6 \%\end{array}$ & $\begin{array}{l}\text { Accroître l'offre pour } \\
\text { répondre à la demande } \\
\text { croissante des } \\
\text { consommateurs }\end{array}$ & $\begin{array}{l}\text { Divers } \\
\text { produits } \\
\text { alimentaires }\end{array}$ & $1^{\mathrm{er}}$ juin 2008 & 31 déc. 2008 & -- & 578 \\
\hline M2 & $\begin{array}{l}\text { Suppression de la restitution de la } \\
\text { TVA de } 13 \% \text { à l'exportation }{ }^{2}\end{array}$ & $\begin{array}{l}\text { Réduire les } \\
\text { exportations pour faire } \\
\text { baisser la demande de } \\
\text { céréales }^{3}\end{array}$ & Éthanol & $\begin{array}{l}1^{\mathrm{er}} \text { janv. } \\
2007\end{array}$ & $\begin{array}{l}\text { Toujours en } \\
\text { cours }\end{array}$ & -- & -59 \\
\hline M2 & $\begin{array}{l}\text { Suppression de la restitution à } \\
\text { l'exportation de } 13 \% \text { de la valeur } \\
\text { déclarée au port d'exportation }\end{array}$ & $\begin{array}{l}\text { Enrayer la hausse des } \\
\text { prix et accroître les } \\
\text { approvisionnements } \\
\text { intérieurs }\end{array}$ & $\begin{array}{l}\text { Céréales et } \\
\text { soja, et les } \\
\text { farines et } \\
\text { produits } \\
\text { dérivés qui en } \\
\text { sont tirés }\end{array}$ & 20 déc. 2007 & $\begin{array}{c}\text { Toujours en } \\
\text { cours }\end{array}$ & -- & -916 \\
\hline M2 & $\begin{array}{l}\text { Instauration de taxes à } \\
\text { l'exportation : } 5 \% \text { sur le maïs, le } \\
\text { riz, le sorgho, le millet, le soja et les } \\
\text { farines et tourteaux de soja ; } 10 \% \\
\text { sur les farines de riz et de maïs et } \\
\text { sur l'amidon de maïs ; } 20 \% \text { sur le } \\
\text { blé, le seigle, l'orge et l'avoine ; et } \\
25 \% \text { sur la farine de blé et de seigle } \\
\text { et sur l'amidon de blé. Le } 1^{\text {er }} \\
\text { décembre } 2008 \text {, les taxes à } \\
\text { l'exportation sur le maïs et la farine } \\
\text { et l'amidon de maïs, et sur le } \\
\text { sorgho, le millet, le seigle, l'orge et } \\
\text { l'avoine ont été supprimées. À cette } \\
\text { date, les taxes à l'exportation sur le } \\
\text { blé et le riz ont été ramenées à } 3 \% \\
\text { et celles sur la farine et l'amidon de } \\
\text { blé à } 8 \% \text {. }\end{array}$ & $\begin{array}{l}\text { Réduire la hausse des } \\
\text { prix alimentaires en } \\
\text { décourageant les } \\
\text { exportations }\end{array}$ & $\begin{array}{l}\text { Céréales et } \\
\text { soja et leurs } \\
\text { dérivés }\end{array}$ & $\begin{array}{l}1^{\mathrm{er}} \text { janv. } \\
2008\end{array}$ & 30 juin 2009 & -- & -684 \\
\hline M2 & $\begin{array}{l}\text { Suppression de la restitution de la } \\
\text { TVA à l'exportation - dont les taux } \\
\text { allaient de } 13 \% \text { à } 17 \%\end{array}$ & $\begin{array}{l}\text { Endiguer la hausse des } \\
\text { prix alimentaires }\end{array}$ & $\begin{array}{l}\text { Huiles } \\
\text { végétales }\end{array}$ & 13 juin 2008 & $\begin{array}{c}\text { Toujours en } \\
\text { cours }\end{array}$ & -- & -467 \\
\hline M4 & $\begin{array}{l}\text { Instauration de mesures de contrôle } \\
\text { des prix imposant aux grossistes et } \\
\text { aux détaillants de déclarer les } \\
\text { augmentations ponctuelles égales } \\
\text { ou supérieures à } 5 \% \text { et les hausses } \\
\text { cumulées égales ou supérieures à } \\
8 \% \text { sur les prix du mois d'octobre } \\
2007\end{array}$ & $\begin{array}{l}\text { Endiguer la hausse des } \\
\text { prix alimentaires }\end{array}$ & $\begin{array}{c}\text { Céréales } \\
\text { alimentaires, } \\
\text { huiles } \\
\text { végétales, } \\
\text { viande de } \\
\text { porc, de } \\
\text { bœuf, de } \\
\text { mouton, } \\
\text { produits } \\
\text { laitiers et } \\
\text { œuff }\end{array}$ & $\begin{array}{l}26 \text { janv. } \\
2008\end{array}$ & $1^{\text {er }}$ déc. 2008 & -- & -- \\
\hline M6 & $\begin{array}{l}\text { Limitation de l'octroi de } \\
\text { contingents d'exportation }\end{array}$ & $\begin{array}{l}\text { Assurer } \\
\text { l'approvisionnement } \\
\text { intérieur }\end{array}$ & $\begin{array}{l}\text { Maïs, riz et } \\
\text { blé }\end{array}$ & 2007 & $\begin{array}{l}\text { Toujours en } \\
\text { cours }\end{array}$ & -- & -- \\
\hline M6 & $\begin{array}{l}\text { Instauration d'un système de } \\
\text { licences d'exportation }\end{array}$ & $\begin{array}{l}\text { Soumettre à un } \\
\text { plafond le volume des } \\
\text { exportations au cas où } \\
\text { les taxes à } \\
\text { l'exportation ne } \\
\text { seraient pas } \\
\text { suffisamment élevées }\end{array}$ & $\begin{array}{l}\text { Farines de } \\
\text { blé, de maïs } \\
\text { et de riz }\end{array}$ & $\begin{array}{l}1^{\mathrm{er}} \text { janv. } \\
2008\end{array}$ & $\begin{array}{c}\text { Toujours en } \\
\text { cours }\end{array}$ & -- & -- \\
\hline
\end{tabular}


Annexe tableau A.4. Mesures à court terme prises : Chine (suite)

\begin{tabular}{|c|c|c|c|c|c|c|c|}
\hline \multirow[b]{2}{*}{ Étiquette } & \multirow[b]{2}{*}{ Description de la mesure } & \multirow[b]{2}{*}{ Objectif } & \multirow[b]{2}{*}{ Produit } & \multirow{2}{*}{$\begin{array}{l}\text { Date de } \\
\text { début }\end{array}$} & \multirow[b]{2}{*}{ Date de fin ${ }^{9}$} & \multicolumn{2}{|c|}{$\begin{array}{c}\text { Impact } \\
\text { budgétaire } \\
\text { (millions CNY) }\end{array}$} \\
\hline & & & & & & 2007 & 2008 \\
\hline M7 & $\begin{array}{l}\text { Interventions sur le marché grâce } \\
\text { aux stocks publics nationaux }\end{array}$ & Accroître l'offre & Riz et blé & 2007 & 2008 & -- & -- \\
\hline M8 & $\begin{array}{l}\text { Arrêt de toute nouvelle autorisation } \\
\text { d'usine de transformation des } \\
\text { céréales, y compris pour la } \\
\text { production de biocarburants }\end{array}$ & $\begin{array}{l}\text { Soumettre à un } \\
\text { plafond la } \\
\text { consommation } \\
\text { industrielle }\end{array}$ & Céréales & 2007 & 2008 & -- & -- \\
\hline M9 & $\begin{array}{l}\text { Instauration d'un nouveau système } \\
\text { de déclaration des importations de } \\
\text { certains produits agricoles de base } \\
\text { en vrac }\end{array}$ & $\begin{array}{l}\text { Mieux suivre les } \\
\text { évolutions de l'offre } \\
\text { intérieur et des cours } \\
\text { internationaux }\end{array}$ & $\begin{array}{l}\text { Un large } \\
\text { éventail }\end{array}$ & Août 2008 & $\begin{array}{c}\text { Toujours en } \\
\text { cours }\end{array}$ & -- & -- \\
\hline I1 & $\begin{array}{l}\text { La Banque populaire de } \\
\text { Chine (BPC) a développé ses } \\
\text { interventions sur le marché en } \\
\text { accroissant ses émissions de titres et } \\
\text { les rachats de bons. }\end{array}$ & $\begin{array}{l}\text { Réduire } \\
\text { l'augmentation de } \\
\text { l'offre de monnaie } \\
\text { générée par l'achat de } \\
\text { devises par la PBC } \\
\text { afin de maintenir la } \\
\text { stabilité de la monnaie } \\
\end{array}$ & Tous & Début 2007 & Juil. 2008 & -- & -- \\
\hline I1 & $\begin{array}{l}\text { La BPC a relevé de } 8.5 \text { points de } \\
\text { pourcentage le taux de réserves } \\
\text { obligatoires pour les institutions } \\
\text { financières du } 15 \text { janvier } 2007 \text { au } \\
25 \text { septembre } 2008 \text {. Le taux pour les } \\
\text { institutions financières généralistes } \\
\text { a ainsi été porté de } 9 \% \text { à } 17.5 \% \text {. }\end{array}$ & $\begin{array}{l}\text { Réduire la capacité de } \\
\text { création monétaire du } \\
\text { secteur privé }\end{array}$ & Tous & Janv. 2007 & Sept. 2008 & -- & -- \\
\hline I1 & $\begin{array}{l}\text { La BPC a relevé ses taux débiteur et } \\
\text { créditeur à six reprises entre mars } \\
2007 \text { et décembre } 2007 \text {. Le taux } \\
\text { créditeur à un an a ainsi été relevé } \\
\text { de } 162 \text { points de base, de } 2.52 \% \text { à } \\
4.14 \% \text {. }\end{array}$ & $\begin{array}{l}\text { Freiner la demande et } \\
\text { l'inflation }\end{array}$ & Tous & Mars 2007 & Sept. 2008 & -- & -- \\
\hline I1 & $\begin{array}{l}\text { La BPC a porté de } 0.3 \% \text { à } 0.5 \% \text { la } \\
\text { marge de fluctuation journalière du } \\
\text { taux de change CNY/USD sur le } \\
\text { marché interbancaire au comptant, } \\
\text { permettant ainsi une appréciation du } \\
\text { CNY plus rapide qu'auparavant }\end{array}$ & $\begin{array}{l}\text { Favoriser une balance } \\
\text { des paiements plus } \\
\text { équilibrée }\end{array}$ & Tous & 21 mai 2007 & $\begin{array}{c}\text { Toujours en } \\
\text { cours }\end{array}$ & -- & -- \\
\hline $\mathrm{C} 1$ & $\begin{array}{l}\text { Augmentation de } 23 \% \text { en } 2007 \text { des } \\
\text { paiements mensuels dans le cadre } \\
\text { du «minimum vital garanti » } \\
\text { (programme Di Bao en faveur des } \\
\text { ménages urbains), porté de } \\
83 \text { CNY à } 102 \text { CNY par personne et } \\
\text { par mois, et relèvement du seuil } \\
\text { maximal de revenu en dessous } \\
\text { duquel les personnes sont éligibles } \\
\text { pour les paiements. }\end{array}$ & $\begin{array}{l}\text { Compenser la hausse } \\
\text { des denrées } \\
\text { alimentaires et des } \\
\text { carburants }\end{array}$ & Tous & 2007 & 2008 & 2940 & 4000 \\
\hline $\mathrm{P} 1$ & $\begin{array}{l}\text { Augmentation des prix de soutien } \\
\text { minimums de } 9 \%-10 \%^{7}\end{array}$ & $\begin{array}{l}\text { Stimuler la production } \\
\text { intérieure }\end{array}$ & $\mathrm{Riz}$ & 2008 & $\begin{array}{c}\text { Toujours en } \\
\text { cours }\end{array}$ & -- & 3150 \\
\hline $\mathrm{P} 2$ & $\begin{array}{l}\text { Augmentation des prix de soutien } \\
\text { minimums de } 4 \%-7 \%^{7}\end{array}$ & $\begin{array}{l}\text { Stimuler la production } \\
\text { intérieure }\end{array}$ & Blé & 2008 & $\begin{array}{c}\text { Toujours en } \\
\text { cours }\end{array}$ & -- & 2520 \\
\hline $\mathrm{P} 2$ & $\begin{array}{l}\text { Augmentation de } 131 \% \text { des } \\
\text { dépenses au titre du programme de } \\
\text { subventions générales aux intrants }\end{array}$ & $\begin{array}{l}\text { Dédommager les } \\
\text { agriculteurs des } \\
\text { hausses de prix des } \\
\text { carburants, des engrais } \\
\text { et autres intrants } \\
\text { agricoles }\end{array}$ & Céréales & 2008 & $\begin{array}{l}\text { Toujours en } \\
\text { cours }\end{array}$ & -- & 36200 \\
\hline $\mathrm{P} 2$ & $\begin{array}{l}\text { Extension des superficies couvertes } \\
\text { par le programme de subventions } \\
\text { aux semences, aboutissant à une } \\
\text { augmentation de } 82 \% \text { des dépenses }\end{array}$ & $\begin{array}{l}\text { Accroître la } \\
\text { production en } \\
\text { améliorant les } \\
\text { rendements }\end{array}$ & $\begin{array}{l}\text { Céréales et } \\
\text { oléagineux }\end{array}$ & 2008 & $\begin{array}{c}\text { Toujours en } \\
\text { cours }\end{array}$ & -- & 5440 \\
\hline
\end{tabular}


Annexe tableau A.4. Mesures à court terme prises : Chine (suite)

\begin{tabular}{|c|c|c|c|c|c|c|c|}
\hline \multirow[b]{2}{*}{ Étiquette } & \multirow[b]{2}{*}{ Description de la mesure } & \multirow[b]{2}{*}{ Objectif } & \multirow[b]{2}{*}{ Produit } & \multirow{2}{*}{$\begin{array}{c}\text { Date de } \\
\text { début }\end{array}$} & \multirow[b]{2}{*}{ Date de fin ${ }^{9}$} & \multicolumn{2}{|c|}{$\begin{array}{c}\text { Impact } \\
\text { budgétaire } \\
\text { (millions CNY) }\end{array}$} \\
\hline & & & & & & 2007 & 2008 \\
\hline $\mathrm{P} 2$ & $\begin{array}{l}\text { Instauration de droits à } \\
\text { l'exportation de } 20 \% \text { sur les engrais } \\
\text { phosphatés en février } 2008 \text {. Ces } \\
\text { droits ont été portés à } 100 \% \text { et } \\
\text { étendus à toutes les exportations } \\
\text { d'engrais et de produits connexes } \\
\text { entre le } 20 \text { avril et le } 30 \text { septembre } \\
2008 \text { (touchant } 32 \text { lignes tarifaires, } \\
\text { dont les engrais phosphorés, } \\
\text { ammoniaqués, azotés, phosphatés, } \\
\text { potassiques et les engrais } \\
\text { composés). Au début septembre } \\
\text { 2008, les droits de douanes à } \\
\text { l'exportation d'engrais ont été } \\
\text { portés à } 150 \% \text {. }\end{array}$ & $\begin{array}{l}\text { Aider à enrayer la } \\
\text { hausse des prix et à } \\
\text { garantir une récolte de } \\
\text { céréales abondante } \\
\text { l'année considérée. }\end{array}$ & Engrais & 15 fév. 2008 & $\begin{array}{c}24 \text { janv. } \\
2009\end{array}$ & n.d. & n.d. \\
\hline $\mathrm{P} 2$ & $\begin{array}{l}\text { Réduction des droits de douane, } \\
\text { ramenés de } 5 \% \text { à } 2 \%\end{array}$ & $\begin{array}{l}\text { Assurer un } \\
\text { approvisionnement } \\
\text { suffisant aux éleveurs }\end{array}$ & $\begin{array}{l}\text { Tourteaux et } \\
\text { produits } \\
\text { d'alimentatio } \\
\mathrm{n} \text { animale à } \\
\text { base de soja } \\
\text { et d'arachides }\end{array}$ & $1^{\mathrm{er}}$ juin 2008 & 31 déc. 2008 & 15 & -- \\
\hline $\mathrm{P} 3$ & $\begin{array}{l}\text { Augmentation de } 233 \% \text { des } \\
\text { dépenses au titre du programme } \\
\text { pour l'achat de matériel agricole et } \\
\text { extension de celui-ci, des deux tiers } \\
\text { des districts agricoles à la totalité } \\
\text { d'entre eux }\end{array}$ & $\begin{array}{l}\text { Aider à enrayer la } \\
\text { hausse des prix et à } \\
\text { garantir une récolte de } \\
\text { céréales abondante } \\
\text { l'année considérée. }\end{array}$ & $\begin{array}{l}\text { Riz, blé et } \\
\text { maïs }\end{array}$ & 2008 & $\begin{array}{c}\text { Toujours en } \\
\text { cours }\end{array}$ & -- & 2800 \\
\hline P5 & $\begin{array}{l}\text { Annonce que le gouvernement } \\
\text { examinera l'ampleur et la qualité } \\
\text { des plans d'affectation des sols, } \\
\text { mettra en œuvre le système de } \\
\text { réglementation de l'utilisation des } \\
\text { terres, administrera au moyen d'une } \\
\text { loi les terres constructibles pour les } \\
\text { collectifs ruraux et les individus, et } \\
\text { mettra fin au comportement } \\
\text { d'occupation illégale des terres } \\
\text { agricoles et des espaces forestiers. }\end{array}$ & $\begin{array}{l}\text { Aider à atteindre le } \\
\text { seuil minimal de } \\
120 \text { millions } \\
\text { d'hectares de terres } \\
\text { arables }\end{array}$ & Céréales & Mars 2008 & $\begin{array}{c}\text { Toujours en } \\
\text { cours }\end{array}$ & -- & -- \\
\hline
\end{tabular}

«-- » : aucun impact budgétaire ; « n.d. » : non disponible.

1. Les produits alimentaires sont les suivants : porc congelé, poissons congelés (morue, églefin et lieu noir), lactosérum et lactosérum modifié, pistaches, préparations destinées aux nourrissons pour la vente au détail, extraits de malt et levure.

2. Les restitutions à l'exportation et les restitutions de TVA ont fait partie intégrante de la politique d'incitations fiscales mise en œuvre depuis les années 80 pour encourager les exportations de toutes les catégories de produits. Les restitutions à l'exportation pour les produits contenant des intrants agricoles ont été portées en 2007 de $5 \%$ à $11 \%-13 \%$ afin de favoriser l'utilisation d'intrants agricoles dans la production intérieure destinée aux marchés d'exportation.

3. Dans l'industrie chinoise des biocarburants, les céréales sont utilisées pour produire de l'éthanol. Quatre usines utilisent des céréales pour matière première $-80 \%$ de la production est issue du maïs et $20 \%$ du blé et du riz.

4. Les produits agricoles soumis à des contingents d'exportation sont le coton, le maïs, le riz, le blé et le thé. Les exportations de coton, de maïs et de riz relèvent par ailleurs d'un système de commerce d'État.

5. La date de fin correspond au mois où le taux d'intérêt de la politique monétaire a été réduit afin de stimuler l'économie.

6. Ces dépenses ne correspondent qu'à l'augmentation de la part des paiements prise en charge par l'administration centrale. Ce programme bénéficie également de contributions financières des autorités provinciales.

7. Les prix de soutien minimums pour différentes variétés de riz et de blé n'avaient pas changé depuis leur instauration - en 2004 pour le riz et en 2006 pour le blé. Ils ont de nouveau été relevés pour 2009 : de 16\%-17\% pour le riz et de 13\%-15\% pour le blé.

8. Bien que le montant unitaire des subventions soit demeuré identique, les superficies bénéficiant du programme ont très nettement augmenté. Les superficies de blé sont ainsi passées de 6.7 millions d'hectares en 2007 à 13.3 millions en 2008 , et celles de maïs de 2 millions à 13.3 millions d'hectares.

9. La mention « Toujours en cours » indique que la mesure considérée était encore en vigueur au 31 décembre 2009. 
Sources :

FAO (2010), Suivi du marché du riz, divers numéros, FAO, Rome, http://www.fao.org/es/ESC/fr/15/70/highlight 71.html.

GAIN-CH7012 (2007), China: Oilseeds - Annual Report 2007, USDA/FAS, $1^{\mathrm{er}}$ mars.

GAIN-CH7015 (2007), China: Grain and Feed - Annual Report 2007, USDA/FAS, ${ }^{\text {er }}$ mars.

GAIN-CH7093 (2007), China: Agriculture Situation - China Removes Export Rebate on Grains, Soy Beans and Flour Products

USDA/FAS, 18 décembre.

GAIN-CH8001 (2008), China: Agriculture Situation - China Tightens Control on Grain and Flour Exports, USDA/FAS, 14 janvier

GAIN-CH8010 (2008), China: Oilseeds - Annual Report 2008, USDA/FAS, $1^{\mathrm{er}}$ mars.

GAIN-CH8012 (2008), China: Grain and Feed - Annual Report 2008, USDA/FAS, ${ }^{\text {er }}$ mars.

GAIN-CH8025 (2008), China: Agriculture Situation - Increasing Food Prices, USDA/FAS, 21 avril.

GAIN-CH8040 (2008), China: Food and Agricultural Import Regulations and Standards - China reduced tariffs on selected

agricultural commodities, USDA/FAS, 30 mai.

GAIN-CH8054 (2008), China: Agriculture Situation - Value Added Tax Rebate Eliminated for Vegetable Oil Exports, USDA/FAS,

6 juin.

GAIN-CH9013 (2009), China: Grain and Feed - Annual Report 2009, USDA/FAS, 3 mars.

GAIN-CH9030 (2009), China: Oilseeds - Annual Report 2008, USDA/FAS, 15 avril.

Hansen, J., et al. (2009), "Impact of China's agriculture policies on domestic and world commodity markets", document présenté lors de la Conférence de l'Association internationale d'économie agricole, Pékin, Chine, 16-22 août,

http://ageconsearch.umn.edu/bitstream/51704/2/IAAE2009-Ref723-JimHansen.pdf.

Li, Xiande (2008), "Report on Agricultural Policy Developments in China: 2006-2008”, rapport soumis à l'OCDE.

$\mathrm{Li}$, Xiande (2009), "China: Short-term policy responses to higher and volatile food prices", communication présentée au Forum

mondial sur l'agriculture de l'OCDE de 2009, www.oecd.org/agriculture/globalforum/june09.

Lohmar, B. (2009), "China's ongoing agricultural modernisation: Challenges remains after 30 years of reform”, Economic Information Bulletin $n^{\circ} 51$, Service de recherche économique (Economic Research Service), USDA, Washington, DC,

www.ers.usda.gov/Publications/EIB51/EIB51.pdf.

Banque populaire de Chine [BPC] (2008), Annual Report 2007, BPC, Pékin, www.pbc.gov.cn/english/chubanwu/nianbao/2008.asp.

BPC (2009), Annual Report 2008, BPC, Pékin, www.pbc.gov.cn/english/chubanwu/nianbao/2007.asp.

Yang, J., et al. (2008), "Fighting global food price rises in the developing world: the response of China and its effect on domestic and world markets", Agricultural Economics, vol.38, supplément, pp. 453-464, http://www3.interscience.wiley.com/cgibin/fulltext/121554071/PDFSTART.

Banque mondiale (2009), From poor areas to poor people: China's evolving poverty reduction agenda - An assessment of poverty and inequality in China, Banque mondiale, Pékin, mars, http://go.worldbank.org/2RAHXHWLOO.

OMC (2008), Examen des politiques commerciales : Chine, WT/TPR/S/199, OMC, Genève, 16 avril, http://www.wto.org/french/tratop f/tpr f/tp299 f.htm. 
Annexe tableau A.5. Mesures à court terme prises : Inde

\begin{tabular}{|c|c|c|c|c|c|c|c|}
\hline \multirow[b]{2}{*}{ Étiquette } & \multirow[b]{2}{*}{ Description de la mesure } & \multirow[b]{2}{*}{ Objectif } & \multirow[b]{2}{*}{ Produit } & \multirow{2}{*}{$\begin{array}{l}\text { Date de } \\
\text { début }\end{array}$} & \multirow[b]{2}{*}{ Date de fin ${ }^{12}$} & \multicolumn{2}{|c|}{$\begin{array}{c}\text { Impact } \\
\text { budgétaire } \\
\text { (milliards INR) } \\
\end{array}$} \\
\hline & & & & & & 2007 & 2008 \\
\hline M1 & $\begin{array}{l}\text { Réduction des droits de douane, } \\
\text { ramenés de } 10 \% \text { à } 0 \%\end{array}$ & $\begin{array}{l}\text { Endiguer la } \\
\text { hausse des prix } \\
\text { alimentaires } \\
\end{array}$ & Légumes secs & 8 juin 2006 & $\begin{array}{l}31 \text { mars } \\
2009\end{array}$ & 4 & 5 \\
\hline M1 & $\begin{array}{l}\text { Réduction des droits de douane, } \\
\text { ramenés de } 50 \% \text { à } 5 \% \text { pour les } \\
\text { importations du secteur privé, puis à } 0 \% \\
\text { à compter du } 9 \text { septembre } 2006 \text {. Les } \\
\text { importations de blé des organismes } \\
\text { publics (tels que la Société de commerce } \\
\text { d'État) sont déjà exonérées. Le secteur } \\
\text { privé est obligé de se conformer aux } \\
\text { mêmes normes de qualité que celles } \\
\text { appliquées par les organismes } \\
\text { publiques. }\end{array}$ & $\begin{array}{l}\text { Atténuer la } \\
\text { pénurie de blé sur } \\
\text { le marché } \\
\text { intérieur et faire } \\
\text { ainsi baisser les } \\
\text { prix }\end{array}$ & Blé & 28 juin 2006 & $\begin{array}{c}\text { Toujours en } \\
\text { cours }\end{array}$ & n.d. & n.d. \\
\hline M1 & $\begin{array}{l}\text { Réduction des droits de douane, } \\
\text { ramenés de } 50 \% \text { à } 0 \%\end{array}$ & $\begin{array}{l}\text { Encourager les } \\
\text { importations à des } \\
\text { prix en hausse } \\
\text { régulière }\end{array}$ & Maïs & $\begin{array}{l}25 \text { janv. } \\
2007\end{array}$ & 31 déc. 2007 & 0.04 & -- \\
\hline M1 & $\begin{array}{l}\text { Réduction des droits de douane, } \\
\text { ramenés de } 75 \%-80 \% \text { à } 40 \%-45 \% \text { le } \\
23 \text { juillet } 2007 \text {, puis à } 20 \% \text { le } 21 \text { mars } \\
2008 \text {, et enfin à } 0 \% \text { à compter du } \\
1^{\text {er }} \text { avril } 2008 \text {. Les droits de douane sur } \\
1^{\prime} \text { huile de soja brute ont été remontés à } \\
20 \% \text { en novembre } 2008 \text { mais de } \\
\text { nouveau ramenés à } 0 \% \text { le } 19 \text { mars } \\
2009 .^{2}\end{array}$ & $\begin{array}{l}\text { Encourager les } \\
\text { importations à des } \\
\text { prix en hausse } \\
\text { régulière }\end{array}$ & $\begin{array}{l}\text { Huiles de } \\
\text { palme, de } \\
\text { soja et de } \\
\text { tournesol, } \\
\text { brutes }\end{array}$ & $\begin{array}{l}23 \text { juillet } \\
2007\end{array}$ & $\begin{array}{c}\text { Toujours en } \\
\text { cours }\end{array}$ & 23 & 70 \\
\hline M1 & $\begin{array}{l}\text { Réduction des droits de douane, } \\
\text { ramenés de } 36 \% \text { à } 0 \%\end{array}$ & $\begin{array}{l}\text { Encourager les } \\
\text { importations à des } \\
\text { prix en hausse } \\
\text { régulière }\end{array}$ & Farine de blé & 2 janv. 2008 & $\begin{array}{l}31 \text { mars } \\
2009\end{array}$ & -- & 4 \\
\hline M1 & $\begin{array}{l}\text { Réduction des droits de douane ramenés } \\
\text { de } 70 \%-80 \% \text { à } 0 \% \text { - initialement } \\
\text { jusqu'au } 31 \text { mars } 2009 \text {, mais la mesure a } \\
\text { été prorogée jusqu'au } 30 \text { septembre } \\
2010\end{array}$ & $\begin{array}{l}\text { Encourager les } \\
\text { importations à des } \\
\text { prix en hausse } \\
\text { régulière }\end{array}$ & Riz & $\begin{array}{l}21 \text { mars } \\
2008\end{array}$ & $\begin{array}{c}\text { Toujours en } \\
\text { cours }\end{array}$ & -- & 0 \\
\hline M1 & $\begin{array}{l}\text { Réduction des droits de douane, } \\
\text { ramenés de } 40 \%-75 \% \text { à } 20 \%-27.5 \%, \\
\text { puis à } 7.5 \% \text { à compter du } 1^{\mathrm{er}} \text { avril } 2008 \text {. }\end{array}$ & $\begin{array}{l}\text { Encourager les } \\
\text { importations à des } \\
\text { prix en hausse } \\
\text { régulière }\end{array}$ & $\begin{array}{l}\text { Huiles de } \\
\text { palme, de } \\
\text { soja et de } \\
\text { tournesol, } \\
\text { raffinées }\end{array}$ & $\begin{array}{l}21 \text { mars } \\
2008\end{array}$ & $\begin{array}{c}\text { Toujours en } \\
\text { cours }\end{array}$ & -- & 19 \\
\hline M2 & $\begin{array}{l}\text { Instauration d'une taxe à l'exportation } \\
\text { de } 8000 \text { INR par tonne (environ } \\
\text { 200 USD) }\end{array}$ & $\begin{array}{l}\text { Décourager les } \\
\text { exportations }\end{array}$ & Riz basmati & 29 avr. 2008 & $\begin{array}{l}20 \text { janv. } \\
2009\end{array}$ & -- & n.d. \\
\hline M5 & $\begin{array}{l}\text { La Société de commerce d'État de } \\
\text { l'Inde a importé, au travers d'appels } \\
\text { d'offre publics, } 5 \text { millions de tonnes de } \\
\text { blé en } 2006 / 07 \text { et } 2 \text { millions de tonnes } \\
\text { en } 2007 / 08\end{array}$ & $\begin{array}{l}\text { Stabiliser les prix } \\
\text { sur le marché } \\
\text { intérieur }\end{array}$ & Blé & 2006 & 2008 & & \\
\hline M5 & $\begin{array}{l}\text { Instauration d'une subvention aux } \\
\text { importations d'huiles comestibles par } \\
\text { les sociétés du secteur public }\end{array}$ & $\begin{array}{l}\text { Encourager les } \\
\text { importations à des } \\
\text { prix en hausse } \\
\text { régulière }\end{array}$ & $\begin{array}{c}\text { Huiles } \\
\text { comestibles }\end{array}$ & 2008 & $\begin{array}{c}\text { Toujours en } \\
\text { cours }\end{array}$ & -- & 5 \\
\hline M6 & $\begin{array}{l}\text { Instauration d'une interdiction des } \\
\text { exportations de tous les légumes secs, } \\
\text { sauf celles de kabuli chana (pois } \\
\text { chiches) }\end{array}$ & $\begin{array}{l}\text { Limiter la hausse } \\
\text { des prix }\end{array}$ & Légumes secs & 22 juin 2006 & $\begin{array}{l}31 \text { mars } \\
2009\end{array}$ & -- & -- \\
\hline M6 & $\begin{array}{l}\text { Instauration d'une interdiction des } \\
\text { exportations }\end{array}$ & $\begin{array}{l}\text { Limiter la hausse } \\
\text { des prix }\end{array}$ & $\begin{array}{l}\text { Poudres de } \\
\text { lait }\end{array}$ & $1^{\mathrm{er}}$ fév. 2007 & $\begin{array}{l}30 \text { sept. } \\
2007\end{array}$ & -- & -- \\
\hline
\end{tabular}


Annexe tableau A.5. Mesures à court terme prises : Inde (suite)

\begin{tabular}{|c|c|c|c|c|c|c|c|}
\hline \multirow[b]{2}{*}{ Étiquette } & \multirow[b]{2}{*}{ Description de la mesure } & \multirow[b]{2}{*}{ Objectif } & \multirow[b]{2}{*}{ Produit } & \multirow{2}{*}{$\begin{array}{c}\text { Date de } \\
\text { début }\end{array}$} & \multirow[b]{2}{*}{ Date de fin ${ }^{12}$} & \multicolumn{2}{|c|}{$\begin{array}{c}\text { Impact } \\
\text { budgétaire } \\
\text { (milliards INR) }\end{array}$} \\
\hline & & & & & & 2007 & 2008 \\
\hline M6 & $\begin{array}{l}\text { Instauration d'une interdiction des } \\
\text { exportations - bien que des ventes } \\
\text { d'État à État aient été autorisées. }{ }^{3} \\
\text { Celles de semences de blé ont été } \\
\text { autorisées à partir du } 18 \text { septembre } \\
2008 \text {. }\end{array}$ & \multirow[t]{2}{*}{$\begin{array}{l}\text { Limiter la hausse } \\
\text { des prix }\end{array}$} & \multirow[t]{2}{*}{$\begin{array}{l}\text { Blé et } \\
\text { produits du } \\
\text { blé }\end{array}$} & 9 fév. 2007 & 2 juin 2009 & -- & -- \\
\hline M6 & $\begin{array}{l}\text { Instauration d'un contingent } \\
\text { d'exportation de } 650000 \text { tonnes }\end{array}$ & & & 3 juin 2009 & $\begin{array}{c}31 \text { mars } \\
2010\end{array}$ & -- & -- \\
\hline M6 & $\begin{array}{l}\text { Interdiction des exportations - bien que } \\
\text { les exportations d'aide alimentaire } \\
\text { bénéficient d'une dérogation }\end{array}$ & \multirow{3}{*}{$\begin{array}{l}\text { Limiter la hausse } \\
\text { des prix }\end{array}$} & \multirow{3}{*}{$\begin{array}{l}\text { Riz autre que } \\
\text { le basmati }\end{array}$} & 9 oct. 2007 & 30 oct. 2007 & -- & -- \\
\hline M6 & $\begin{array}{l}\text { Instauration d'un prix minimum à } \\
\text { l'exportation (PME) de } 425 \text { USD la } \\
\text { tonne. Son montant a été porté à } 500 \\
\text { USD le } 27 \text { décembre } 2007 \text {, puis à } \\
650 \text { USD le } 5 \text { mars } 2008 \text { et à } 1000 \text { USD } \\
\text { le } 27 \text { mars } 2008 \text {. }\end{array}$ & & & 31 oct. 2007 & $\begin{array}{l}31 \text { mars } \\
2008\end{array}$ & -- & -- \\
\hline M6 & $\begin{array}{l}\text { Rétablissement de l'interdiction des } \\
\text { exportations }{ }^{4}\end{array}$ & & & $1^{\text {er }}$ avril 2008 & $8 \begin{array}{c}\text { Toujours en } \\
\text { cours }\end{array}$ & -- & -- \\
\hline M6 & $\begin{array}{l}\text { Instauration d'un PME de } 950 \text { USD par } \\
\text { tonne. Son montant a été porté à } 1100 \\
\text { USD le } 27 \text { mars } 2008 \text { et à } 1200 \text { USD le } \\
1^{\text {er }} \text { avril } 2008 \text {. Il a été ramené à } 1100 \\
\text { USD le } 20 \text { janvier } 2009 \text { et à } 900 \text { USD le } \\
7 \text { septembre } 2009 \text {. }\end{array}$ & $\begin{array}{l}\text { Limiter la hausse } \\
\text { des prix }\end{array}$ & Riz basmati & 5 mars 2008 & $\begin{array}{c}\text { Toujours en } \\
\text { cours }\end{array}$ & -- & -- \\
\hline M6 & $\begin{array}{l}\text { Restriction des exportations aux ports de } \\
\text { Mundra et de Pipavav uniquement. } \\
\text { Extension de cette mesure le } 1^{\text {er }} \text { avril } \\
2008 \text { en vue de permettre les } \\
\text { exportations via Kandla, Kakinada, } \\
\text { Kolkata et Mumbai JNPT. }\end{array}$ & $\begin{array}{l}\text { Limiter la hausse } \\
\text { des prix }\end{array}$ & Riz basmati & $\begin{array}{l}17 \text { mars } \\
2008\end{array}$ & $\begin{array}{c}\text { Toujours en } \\
\text { cours }\end{array}$ & -- & -- \\
\hline M6 & $\begin{array}{l}\text { Instauration d'une interdiction des } \\
\text { exportations }\end{array}$ & $\begin{array}{l}\text { Limiter la hausse } \\
\text { des prix }\end{array}$ & Maïs & $\begin{array}{l}3 \text { juillet } \\
2008\end{array}$ & 15 oct. 2008 & -- & -- \\
\hline M7 & Déblocage des stocks publics & $\begin{array}{l}\text { Contenir les } \\
\text { hausses de prix }\end{array}$ & Riz et blé & 2006 & $\begin{array}{c}\text { Toujours en } \\
\text { cours }\end{array}$ & -- & -- \\
\hline M8 & $\begin{array}{l}\text { Restrictions au transport de blé par } \\
\text { chemin de fer par les négociants privés }\end{array}$ & $\begin{array}{l}\text { Maximiser la part } \\
\text { de la récolte } \\
\text { achetée par l'État }\end{array}$ & Blé & Avril 2008 & Mai 2008 & -- & -- \\
\hline M8 & $\begin{array}{l}\text { Décret du gouvernement central en vertu } \\
\text { de la loi de } 1955 \text { sur les produits de } \\
\text { première nécessité pour permettre aux } \\
\text { autorités des États d'imposer des limites } \\
\text { aux stocks détenus par le secteur privé } \\
\text { (grossistes et détaillants) - sauf pour les } \\
\text { stocks d'huiles importées }\end{array}$ & $\begin{array}{l}\text { Éviter } \\
\text { l'accaparement et } \\
\text { faciliter les achats } \\
\text { publics malgré les } \\
\text { tensions du côté } \\
\text { de l'offre }\end{array}$ & $\begin{array}{l}\text { Huiles } \\
\text { végétales } \\
\text { comestibles, } \\
\text { légumes secs, } \\
\text { riz et blé }\end{array}$ & 29 août 2006 & $6 \begin{array}{c}\text { Toujours en } \\
\text { cours }^{5}\end{array}$ & -- & -- \\
\hline M9 & $\begin{array}{l}\text { Retrait de la liste des produits faisant } \\
\text { l'objet de transactions à terme }\end{array}$ & Réduire les & $\begin{array}{c}\text { Deux variétés } \\
\text { de } \\
\text { légumineuses } \\
\text { - Urad } \\
\text { (haricot } \\
\text { mungo) and } \\
\text { Tur (pois } \\
\text { cajan) }\end{array}$ & $\begin{array}{l}23 \text { janv. } \\
2007\end{array}$ & $\begin{array}{c}\text { Toujours en } \\
\text { cours }\end{array}$ & -- & -- \\
\hline M9 & $\begin{array}{l}\text { Retrait de la liste des produits faisant } \\
\text { l'objet de transactions à terme }\end{array}$ & $\begin{array}{l}\text { pressions } \\
\text { spéculatives sur } \\
\text { les prix }\end{array}$ & $\mathrm{Riz}$ & 28 fév. 2007 & $\begin{array}{c}\text { Toujours en } \\
\text { cours }\end{array}$ & -- & -- \\
\hline M9 & $\begin{array}{l}\text { Retrait de la liste des produits faisant } \\
\text { l'objet de transactions à terme }\end{array}$ & & Blé & 28 fév. 2007 & 15 mai 2009 & -- & -- \\
\hline M9 & $\begin{array}{l}\text { Retrait de la liste des produits faisant } \\
\text { l'objet de transactions à terme }\end{array}$ & & $\begin{array}{l}\text { Huile de soja, } \\
\text { caoutchouc, } \\
\text { pommes de } \\
\text { terre et pois } \\
\text { chiches }\end{array}$ & 7 mai 2008 & 30 nov. 2008 & -- & -- \\
\hline
\end{tabular}


Annexe tableau A.5. Mesures à court terme prises : Inde (suite)

\begin{tabular}{|c|c|c|c|c|c|c|c|}
\hline \multirow[b]{2}{*}{ Étiquette } & \multirow[b]{2}{*}{ Description de la mesure } & \multirow[b]{2}{*}{ Objectif } & \multirow[b]{2}{*}{ Produit } & \multirow{2}{*}{$\begin{array}{c}\text { Date de } \\
\text { début }\end{array}$} & \multirow[b]{2}{*}{ Date de fin ${ }^{12}$} & \multicolumn{2}{|c|}{$\begin{array}{c}\text { Impact } \\
\text { budgétaire } \\
\text { (milliards INR) }\end{array}$} \\
\hline & & & & & & 2007 & 2008 \\
\hline M9 & $\begin{array}{l}\text { Création d'une réserve stratégique de } \\
5 \text { millions de tonnes de céréales } \\
\text { alimentaires }-3 \text { millions de tonnes de } \\
\text { blé et } 2 \text { millions de tonnes de riz. Ces } \\
\text { stocks viennent s'ajouter aux stocks } \\
\text { régulateurs déjà constitués pour les } \\
\text { besoins du système de distribution } \\
\text { publique (PDS). }\end{array}$ & $\begin{array}{l}\text { Renforcer la } \\
\text { sécurité } \\
\text { alimentaire et } \\
\text { satisfaire les } \\
\text { besoins les plus } \\
\text { urgents }\end{array}$ & Riz, blé & $\begin{array}{l}25 \text { avril } \\
2008\end{array}$ & $\begin{array}{c}\text { Toujours en } \\
\text { cours }\end{array}$ & -- & -- \\
\hline I1 & $\begin{array}{l}\text { La Reserve Bank of India (RBI) a relevé } \\
\text { de } 400 \text { points de base (de } 5 \% \text { à } 9 \% \text { ) le } \\
\text { taux de réserves obligatoires entre } \\
\text { décembre } 2006 \text { et août } 2008^{7}\end{array}$ & $\begin{array}{l}\text { Maîtriser } \\
\text { l'expansion } \\
\text { monétaire }\end{array}$ & Tous & 23 déc. 2006 & 11 oct. 2008 & -- & -- \\
\hline I1 & $\begin{array}{l}\text { La RBI a relevé son taux débiteur - le } \\
\text { taux auquel elle prête aux banques - de } \\
125 \text { points de base (de } 7.75 \% \text { à } 9 \% \text { ) } \\
\text { entre juin } 2008 \text { et août } 2008^{7}\end{array}$ & $\begin{array}{l}\text { Maîtriser } \\
\text { l'expansion } \\
\text { monétaire }\end{array}$ & Tous & 12 juin 2008 & 20 oct. 2008 & -- & -- \\
\hline $\mathrm{C} 2$ & $\begin{array}{l}\text { Maintenir les prix de vente } \\
\text { subventionnés des produits } \\
\text { commercialisés au travers du système de } \\
\text { distribution publique }\end{array}$ & $\begin{array}{l}\text { Maintenir } \\
\text { l'accessibilité } \\
\text { pour les plus } \\
\text { pauvres } \\
\end{array}$ & Blé et riz & 2007 & $\begin{array}{l}\text { Toujours en } \\
\text { cours }\end{array}$ & 74 & 198 \\
\hline $\mathrm{C} 2$ & $\begin{array}{l}\text { Lancement de la distribution au travers } \\
\text { du PDS d'huiles comestibles } \\
\text { subventionnées ( } 1 \text { million de tonnes à } \\
\text { un taux de subvention de } 15 \text { INR par kg) } \\
\text { à la population en-deçà du seuil de } \\
\text { pauvreté }\end{array}$ & $\begin{array}{l}\text { Accroître } \\
\text { l'accessibilité } \\
\text { pour les plus } \\
\text { pauvres }\end{array}$ & $\begin{array}{l}\text { Huiles } \\
\text { comestibles }\end{array}$ & $\begin{array}{l}17 \text { juillet } \\
2008\end{array}$ & $\begin{array}{c}\text { Toujours en } \\
\text { cours }\end{array}$ & -- & 15 \\
\hline $\mathrm{P} 1$ & $\begin{array}{l}\text { Relèvement du prix de soutien } \\
\text { minimum (PSM) pour les produits } \\
\text { achetés par la Food Corporation of } \\
\text { India (FCI), de } 6400 \text { INR la tonne en } \\
2005 / 06 \text { à } 7000 \text { INR en } 2006 / 07,8500 \\
\text { INR en } 2007 / 08,10000 \text { INR en } \\
2008 / 09 \text { et } 10800 \text { INR en } 2009 / 10^{9}\end{array}$ & $\begin{array}{l}\text { Reconstituer les } \\
\text { stocks de réserve }\end{array}$ & Blé & $1^{\mathrm{er}}$ avril 2006 & $6 \begin{array}{c}\text { Toujours en } \\
\text { cours }\end{array}$ & 9 & 77 \\
\hline $\mathrm{P} 1$ & $\begin{array}{l}\text { Relèvement du PSM pour les produits } \\
\text { achetés par la FCI, de } 5700 \text { INR la } \\
\text { tonne de riz paddy (commun) en } \\
2005 / 06 \text { à } 6200 \text { INR en } 2006 / 07,7450 \\
\text { INR en } 2007 / 08,9000 \text { INR en } 2008 / 09 \\
\text { et } 10000 \text { INR en } 2009 / 10^{9}\end{array}$ & $\begin{array}{l}\text { Reconstituer les } \\
\text { stocks de réserve }\end{array}$ & Riz & $1^{\mathrm{er}}$ oct. 2006 & $\begin{array}{c}\text { Toujours en } \\
\text { cours }\end{array}$ & 26 & 101 \\
\hline $\mathrm{P} 1$ & $\begin{array}{l}\text { Relèvement des prix de soutien } \\
\text { minimum }^{10}\end{array}$ & $\begin{array}{l}\text { Égaler les hausses } \\
\text { accordées pour les } \\
\text { céréales } \\
\text { alimentaires }\end{array}$ & $\begin{array}{l}\text { Céréales } \\
\text { secondaires et } \\
\text { oléagineux }\end{array}$ & 2006 & $\begin{array}{l}\text { Toujours en } \\
\text { cours }\end{array}$ & n.d. & n.d. \\
\hline $\mathrm{P} 2$ & $\begin{array}{l}\text { Maintien à un niveau constant du prix } \\
\text { des engrais malgré la hausse des cours } \\
\text { mondiaux. En outre, du fait d'un } \\
\text { nouveau système de tarification basé sur } \\
\text { la teneur en nutriments, les prix de } \\
\text { divers engrais complexes ont été réduits } \\
\text { de } 18 \% \text { en moyenne. }\end{array}$ & $\begin{array}{l}\text { Fournir aux } \\
\text { exploitants des } \\
\text { engrais à des prix } \\
\text { raisonnables et } \\
\text { assurer aux } \\
\text { producteurs } \\
\text { d'engrais un taux } \\
\text { de rentabilité } \\
\text { raisonnable }\end{array}$ & Tous & Juil. 2008 & $\begin{array}{l}\text { Toujours en } \\
\text { cours }\end{array}$ & 80 & 534 \\
\hline $\begin{array}{l}\mathrm{P} 2, \mathrm{P} 3 \text { et } \\
\text { P4 }\end{array}$ & $\begin{array}{l}\text { Lancement de la Mission nationale pour } \\
\text { la sécurité alimentaire }\end{array}$ & $\begin{array}{l}\text { Accroître la } \\
\text { production de } \\
\text { manière durable }\end{array}$ & $\begin{array}{l}\text { Riz, blé et } \\
\text { légumes secs }\end{array}$ & Août 2007 & $\begin{array}{c}\text { Toujours en } \\
\text { cours }\end{array}$ & -- & 9 \\
\hline
\end{tabular}


«-- » : aucun impact budgétaire ; « n.d. » : non disponible.

1. On ne dispose pas d'informations sur les quantités importées par les négociants privés, mais elles sont probablement négligeables. Des exigences phytosanitaires élevées accroissent les coûts de nettoyage et le risque de rejet de la cargaison au port de destination en Inde. Aussi le prix à l'importation du blé est-il plus élevé que dans d'autres pays pour des qualités de blé comparables.

2. En outre, les prix de référence utilisés pour calculer la valeur des droits de douane sur les huiles comestibles ont été maintenus à leurs niveaux de septembre 2006

3. L'interdiction des exportations sur le blé était initialement destinée à prendre fin le 31 décembre 2007 mais a été prorogée pour une période indéfinie le 8 octobre 2007. Le gouvernement a autorisé l'exportation de 2 million de tonnes en 2008 mais cela n'a pas donné lieu à de vastes exportations commerciales, mais uniquement à des envois humanitaires d'ampleur limitée à destination de l'Afghanistan, des Maldives, du Myanmar et du Népal.

4. Bien qu'elle ne soit pas génétiquement liée au riz basmati, à compter du 5 septembre 2008, l'exportation de la variété de riz parfumé à long grain Pusa-1121 a été autorisée dans les mêmes conditions que pour le riz basmati. En octobre 2008, l'exportation de 55000 tonnes vers le Cameroun, le Ghana, le Nigéria et le Sénégal au travers de la Société de commerce d'État de l'Inde a été autorisée. Le 7 mai 2009, le gouvernement a autorisé l'exportation de 1 million de tonnes par les entreprises d'État à destination de 21 pays.

5. À l'exception du blé, pour lequel le décret a expiré le 30 mars 2009.

6. Les niveaux souhaités des stocks régulateurs « en fin de campagne » nécessaires pour alimenter le système PDS et les autres programmes d'aide sociale sont de 4 millions de tonnes de blé et de 5.2 millions de tonnes de riz. Le coût budgétaire de l'achat des produits destinés à cette réserve stratégique est pris en considération dans l'estimation des coûts imposés par le relèvement du PSM pour le blé et pour le riz.

7. La date de fin correspond au mois où le taux de réserves obligatoires a été réduit afin de stimuler l'économie.

8. Le gouvernement n'a pas augmenté les prix de vente subventionnés du riz et du blé mis sur le marché par l'intermédiaire du PDS depuis le $1^{\text {er }}$ juillet 2002. Depuis cette même date, les prix de soutien ont été relevés de plus de $80 \%$ dans le cas du riz et de plus de $70 \%$ dans celui du blé. Cela a contribué à une sensible augmentation de la subvention des denrées alimentaires qui couvre la différence entre le coût d'achat et de distribution des céréales alimentaires et leur prix de vente aux bénéficiaires.

9. Ces prix tiennent comptes des primes incitatives qui sont parfois annoncées au cours de la campagne agricole, en sus du PSM initialement annoncé. Le PSM a été relativement stable pendant les six campagnes agricoles précédentes.

10. Les prix de soutien minimum pour ces produits demeurent inférieurs aux prix du marché, de sorte que les quantités achetées sont demeurées très modestes.

11. La Mission s'efforce d'y parvenir en comblant l'écart de rendement grâce à la diffusion des meilleures technologies et des meilleures pratiques de gestion des exploitations afin d'assurer la sécurité alimentaire. Ses objectifs sont notamment d'accroître la production de riz de 10 millions de tonnes, celle de blé de 8 millions de tonnes et celle de légumes secs de 2 millions de tonnes en 2011-12.

12. La mention « Toujours en cours » indique que la mesure considérée était encore en vigueur au 31 décembre 2009.

Sources :

FAO (2010), Suivi du marché du riz, divers numéros, FAO, Rome, www.fao.org/es/ESC/fr/15/70/highlight 71.html.

GAIN-IN8015 (2008), India: Grain and Feed - Annual Report 2008, USDA/FAS, 20 février.

GAIN-IN8047 (2008), India: Oilseeds - Annual Report 2008, USDA/FAS, 19 mai.

GAIN-IN9025 (2009), India: Grain and Feed - Annual Report 2009, USDA/FAS, 20 février.

GAIN-IN9051 (2009), India: Oilseeds - Annual Report 2009, USDA/FAS, 16 avril.

GAIN-IN1011 (2010), India: Grain and Feed - Annual Report 2010, USDA/FAS, 17 février.

Kaur, S. (2008), "India's Agricultural Policy Developments: 2006-2008”, rapport soumis à l'OCDE.

Kaur, S. (2009), "India: Short-term responses to higher food prices", communication présentée au Forum mondial sur l'agriculture de l'OCDE de 2009, www.oecd.org/agriculture/globalforum/june09.

Ministère de l'Agriculture (2007), National Food Security Mission: Operational Guidelines, Direction de l'Agriculture et de la

Coopération, New Dehli, http://agricoop.nic.in/NFSM/NFSM.pdf.

Ministère des Finances [MOF] (2008), Economic Survey 2007-08, MOF, New Delhi, http://indiabudget.nic.in/es2007-08/esmain.htm.

Ministère des Finances (2009), Economic Survey 2008-09, MOF, New Delhi, http://indiabudget.nic.in/es2008-09/esmain.htm.

Ministère des Finances (2010), Union Budget, diverses années, MOF, New Delhi, http://indiabudget.nic.in/previousub.htm.

Persaud, S. et M. Landes (2006), "The role of policy and industry structure in India's oilseed market", Economic Research Report $\mathrm{n}^{\circ}$ 17, USDA, ERS, Washington, DC, www.ers.usda.gov/publications/ERR17/ERR17.pdf.

Shikha, J. P. V. Srinivasan et M. Landes (2007), "Indian wheat and rice sector policies and the implications of reform", Economic Research Report n 41, USDA, ERS, Washington, DC, www.ers.usda.gov/publications/err41/err41.pdf.

Slayton, T. (2009), "Rice Price Forensics: How Asian Governments Carelessly Set the World rice Market on Fire", CGD Working

Paper, $\mathrm{n}^{\circ}$ 163, Center for Global Development, Washington, DC, www.cgdev.org/content/publications/detail/1421260/.

OMC (2007), Examen des politiques commerciales : Inde, WT/TPR/S/182, OMC, Genève, 18 avril,

http://www.wto.org/french/tratop f/tpr f/tp283 f.htm. 
Annexe tableau A.6. Mesures à court terme prises : Indonésie

\begin{tabular}{|c|c|c|c|c|c|c|c|}
\hline \multirow[b]{2}{*}{ Étiquette } & \multirow[b]{2}{*}{ Description de la mesure } & \multirow[b]{2}{*}{ Objectif } & \multirow[b]{2}{*}{ Produit } & \multirow{2}{*}{$\begin{array}{c}\text { Date de } \\
\text { début }\end{array}$} & \multirow[b]{2}{*}{ Date de fin ${ }^{11}$} & \multicolumn{2}{|c|}{$\begin{array}{l}\text { Impact budgétaire } \\
\text { (milliards IDR) }\end{array}$} \\
\hline & & & & & & 2007 & 2008 \\
\hline M1 & $\begin{array}{l}\text { Réduction des droits de douane, de } 450 \\
\text { IDR/kg à } 200 \mathrm{IDR} / \mathrm{kg}^{1}\end{array}$ & $\begin{array}{l}\text { Réduire le coût } \\
\text { des importations } \\
\text { effectuées par le } \\
\text { Bulog }\end{array}$ & Riz & Mars 2007 & Mai 2007 & 25 & -- \\
\hline M1 & Suppression des droits de douane de $5 \%$ & $\begin{array}{l}\text { Défendre le } \\
\text { pouvoir d'achat } \\
\text { amoindri des } \\
\text { consommateurs }\end{array}$ & Farine de blé & $\begin{array}{l}21 \text { janv. } \\
2008\end{array}$ & $\begin{array}{c}28 \text { janv. } \\
2009\end{array}$ & -- & 132 \\
\hline M1 & $\begin{array}{l}\text { Suppression des droits de douane de } \\
10 \%\end{array}$ & $\begin{array}{l}\text { Contrebalancer la } \\
\text { flambée des prix }\end{array}$ & Soja & $\begin{array}{l}14 \text { janv. } \\
2008\end{array}$ & $\begin{array}{l}14 \text { juillet } \\
2008\end{array}$ & -- & 338 \\
\hline M2 & $\begin{array}{l}\text { Augmentation des prix à l'exportation } \\
\text { de référence (fixés tous les mois) et des } \\
\text { taxes à l'exportation sur l'huile de } \\
\text { palme brute et ses dérivés. }{ }^{2} \text { Par } \\
\text { exemple, le prix de référence pour } \\
\text { l'huile de palme brute a été porté de } \\
458 \text { USD/t en } 2006 \text { à } 1196 \text { USD/t en } \\
\text { avril } 2008 \text {. La taxe à l'exportation sur } \\
1 \text { 'huile de palme brute a été portée de } \\
1.5 \% \text { en } 2006 \text { à } 6.5 \% \text { en juin } 2007,10 \% \\
\text { en septembre } 2007 \text { et } 20 \% \text { en avril } \\
2008 \text {. La taxe à l'exportation a été } \\
\text { supprimée le } 1^{\text {er }} \text { novembre } 2008 \text {. }\end{array}$ & $\begin{array}{l}\text { Limiter la quantité } \\
\text { d'exportations et } \\
\text { accroître l'offre } \\
\text { d'huile de friture } \\
\text { vendue sur le } \\
\text { marché intérieur }\end{array}$ & $\begin{array}{c}\text { Huile de } \\
\text { palme brute et } \\
\text { ses dérivés }\end{array}$ & Fév. 2007 & Nov. 2008 & n.d. & n.d. \\
\hline M3 & $\begin{array}{l}\text { Lancement d'un programme de } \\
\text { stabilisation des prix prévoyant la } \\
\text { distribution de } 40000 \text { tonnes d'huile de } \\
\text { friture en vrac à prix réduit dans les } \\
\text { principales zones de peuplement }\end{array}$ & $\begin{array}{l}\text { Abaisser les prix } \\
\text { intérieurs de } 23 \%\end{array}$ & $\begin{array}{l}\text { Huile de } \\
\text { friture autre } \\
\text { que de } \\
\text { marque }\end{array}$ & 21 mai 2007 & Nov. 2007 & 160 & -- \\
\hline M3 & Suppression de la TVA de $10 \%^{3}$ & $\begin{array}{l}\text { Accroître le } \\
\text { pouvoir d'achat } \\
\text { des } \\
\text { consommateurs }\end{array}$ & $\begin{array}{l}\text { Huile de } \\
\text { friture autre } \\
\text { que de } \\
\text { marque } \\
\text { présentée } \\
\text { sous } \\
\text { emballage }\end{array}$ & $\begin{array}{c}24 \text { sept. } \\
2007\end{array}$ & Déc. 2008 & 12 & 86 \\
\hline M3 & Suppression de la TVA de $10 \%$ & $\begin{array}{l}\text { Contrebalancer la } \\
\text { flambée des prix }\end{array}$ & Soja & $\begin{array}{l}14 \text { janv. } \\
2008\end{array}$ & 14 juil. 2008 & -- & 742 \\
\hline M3 & Suppression de la TVA de $10 \%$ & $\begin{array}{l}\text { Maintenir la farine } \\
\text { de blé à un prix } \\
\text { abordable pour les } \\
\text { consommateurs }\end{array}$ & $\begin{array}{l}\text { Blé et farine } \\
\text { de blé }\end{array}$ & Fév. 2008 & Janv. 2009 & -- & 2328 \\
\hline M3 & $\begin{array}{l}\text { Instauration d'une subvention aux petits } \\
\text { producteurs de tofu et de tempeh (pain } \\
\text { de soja fermenté) de } 1000 \text { IDR par kg } \\
\text { de soja }\end{array}$ & $\begin{array}{l}\text { Réduire la } \\
\text { pression des prix } \\
\text { sur les } \\
\text { consommateurs à } \\
\text { bas revenus } \\
\end{array}$ & Soja & Avril 2008 & Sept. 2008 & -- & 50 \\
\hline M5 & $\begin{array}{l}\text { Autorisation donnée au Bulog } \\
\text { (Organisation nationale de gestion } \\
\text { logistique des approvisionnements) en } \\
\text { décembre } 2006 \text { d'importer } 0.5 \text { million } \\
\text { de tonnes de riz début } 2007 .{ }^{4} \text { En février } \\
2007 \text {, le Bulog a été autorisé à importer } \\
\text { un autre million de tonnes en } 2007 .{ }^{5}\end{array}$ & $\begin{array}{l}\text { Accroître l'offre } \\
\text { afin d'éviter de } \\
\text { nouvelles hausses } \\
\text { des prises } \\
\text { intérieurs }\end{array}$ & Riz & Janv. 2007 & Déc. 2007 & -- & -- \\
\hline M5 & $\begin{array}{l}\text { Le Bulog est investi du pouvoir de } \\
\text { décider quand il convient d'importer du } \\
\text { riz, en quelles quantités et de quel type. } \\
\text { Le riz importé peut servir à accroître les } \\
\text { réserves publiques, être distribué dans le } \\
\text { cadre du programme Raskin ou être } \\
\text { directement mis sur le marché pour } \\
\text { stabiliser les prix. }\end{array}$ & $\begin{array}{l}\text { Stabiliser les prix } \\
\text { du riz de qualité } \\
\text { inférieure à } \\
4750 \mathrm{IDR} / \mathrm{kg} \\
(0.52 \mathrm{USD} / \mathrm{kg}) \\
\text { jusqu'en janvier } \\
2008\end{array}$ & $\mathrm{Riz}$ & Sept. 2007 & $\begin{array}{l}11 \text { avril } \\
2008\end{array}$ & -- & -- \\
\hline
\end{tabular}


Annexe tableau A.6. Mesures à court terme prises : Indonésie (suite)

\begin{tabular}{|c|c|c|c|c|c|c|c|}
\hline \multirow[b]{2}{*}{ Étiquette } & \multirow[b]{2}{*}{ Description de la mesure } & \multirow[b]{2}{*}{ Objectif } & \multirow[b]{2}{*}{ Produit } & \multirow{2}{*}{$\begin{array}{c}\text { Date de } \\
\text { début }\end{array}$} & \multirow[b]{2}{*}{ Date de fin 11} & \multicolumn{2}{|c|}{$\begin{array}{l}\text { Impact budgétaire } \\
\text { (milliards IDR) }\end{array}$} \\
\hline & & & & & & 2007 & 2008 \\
\hline M5 & $\begin{array}{l}\text { Levée de la norme nationale } \\
\text { indonésienne (établie en juin 1988) } \\
\text { applicable à la farine de blé, supprimant } \\
\text { ainsi l'exigence que le blé importé soit } \\
\text { enrichi en fer, zinc, thiamine, } \\
\text { riboflavine et en acide folique }\end{array}$ & $\begin{array}{l}\text { Accroître les } \\
\text { possibilités } \\
\text { d'approvisionnem } \\
\text { ent }\end{array}$ & Farine de blé & Janv. 2008 & Juil. 2008 & -- & -- \\
\hline M5 & $\begin{array}{l}\text { Approbation de l'importation de farine } \\
\text { de viande et d'os en provenance de deux } \\
\text { nouvelles usines d'équarrissage } \\
\text { américaines en février } 2008 \text {, puis de } \\
\text { deux autres en mars } 2009 \text {, portant leur } \\
\text { nombre total à cinq }\end{array}$ & $\begin{array}{l}\text { Accroître les } \\
\text { possibilités pour } \\
\text { les fabricants } \\
\text { indonésiens de } \\
\text { fourrages de } \\
\text { s'approvisionner à } \\
\text { plus bas coût } \\
\end{array}$ & $\begin{array}{l}\text { Farine de } \\
\text { viande et d'os }\end{array}$ & Fév. 2008 & $\begin{array}{l}\text { Toujours en } \\
\text { cours }\end{array}$ & -- & -- \\
\hline M6 & $\begin{array}{l}\text { Consigne donnée aux producteurs } \\
\text { d'huile de palme brute d'accroître de } \\
50 \% \text { les quantités fournies pour la } \\
\text { fabrication d'huile de friture à l'intérieur } \\
\text { du pays, car les cours mondiaux élevés } \\
\text { les encourageaient à exporter une plus } \\
\text { grande part de leur production }\end{array}$ & $\begin{array}{l}\text { Faire baisser les } \\
\text { prix intérieurs }\end{array}$ & $\begin{array}{l}\text { Huile de } \\
\text { friture autre } \\
\text { que de } \\
\text { marque }\end{array}$ & $1^{\mathrm{er}}$ mai 2007 & Déc. 2007 & -- & -- \\
\hline M6 & $\begin{array}{l}\text { Désignation du Bulog en tant } \\
\text { qu'exportateur exclusif de riz non gluant } \\
\text { et non parfumé, sous réserve } \\
\text { d'approbation par le ministère du } \\
\text { Commerce, et il lui est interdit } \\
\text { d'exporter du riz à moins que ses stocks } \\
\text { n'atteignent au moins } 3 \text { millions de } \\
\text { tonnes, plus du double des niveaux } \\
\text { actuels. Le riz gluant peut être exporté } \\
\text { par n'importe quelle société, sous } \\
\text { réserve d'approbation par le ministère } \\
\text { du Commerce. }\end{array}$ & $\begin{array}{l}\text { Limiter les } \\
\text { exportations et } \\
\text { maîtriser les prix } \\
\text { locaux }\end{array}$ & Riz & $\begin{array}{l}11 \text { avril } \\
2008\end{array}$ & Mai 2009 & -- & -- \\
\hline M7 & $\begin{array}{l}\text { Instruction donnée au BULOG en } \\
\text { décembre } 2006 \text { de débloquer ses stocks } \\
\text { pour les mettre sur le marché - } \\
50000 \text { tonnes en décembre }\end{array}$ & $\begin{array}{l}\text { Faire baisser les } \\
\text { prix intérieurs }\end{array}$ & $\mathrm{Riz}$ & Déc. 2006 & Déc. 2006 & -- & -- \\
\hline M9 & $\begin{array}{l}\text { Annonce de mesures visant à porter le } \\
\text { stock régulateur de } 1 \text { à } 3 \text { millions de } \\
\text { tonnes dans le cadre d'un vaste plan } \\
\text { d'intensification de la production sur } \\
300000 \text { hectares }\end{array}$ & $\begin{array}{l}\text { Assurer la stabilité } \\
\text { des prix intérieurs }\end{array}$ & $\mathrm{Riz}$ & Mai 2008 & $\begin{array}{l}\text { Toujours en } \\
\text { cours }\end{array}$ & -- & -- \\
\hline I1 & $\begin{array}{l}\text { La Banque d'Indonésie a développé ses } \\
\text { opérations sur le marché libre - sa } \\
\text { position passant de } 39 \text { à } 281 \text { billions } \\
\text { IDR }\end{array}$ & $\begin{array}{l}\text { Absorber l'excès } \\
\text { de liquidité }\end{array}$ & Tous & Janv. 2007 & Déc. 2007 & -- & -- \\
\hline I1 & $\begin{array}{l}\text { Vente par la Banque d'Indonésie de } \\
\text { devises prélevées sur ses réserves }{ }^{8}\end{array}$ & $\begin{array}{l}\text { Supprimer la } \\
\text { menace d'une } \\
\text { dépréciation du } \\
\text { taux de change } \\
\end{array}$ & Tous & Janv. 2008 & Juin 2008 & -- & -- \\
\hline I1 & $\begin{array}{l}\text { La Banque d'Indonésie a relevé de } 150 \\
\text { points de base (de } 8 \% \text { à } 9.5 \% \text { ) son taux } \\
\text { d'intérêt de référence entre mai } 2008 \text { et } \\
\text { octobre } 2008^{9}\end{array}$ & $\begin{array}{l}\text { Freiner la } \\
\text { demande et } \\
\text { l'inflation }\end{array}$ & Tous & Mai 2008 & Déc. 2008 & -- & -- \\
\hline $\mathrm{C} 1$ & $\begin{array}{l}\text { Fourniture d'huile de friture } \\
\text { subventionnée ( } 2500 \text { IDR le litre }) \text { à } \\
19.1 \text { millions de ménages pauvres }\end{array}$ & $\begin{array}{l}\text { Atténuer l'impact } \\
\text { de la hausse des } \\
\text { prix sur les plus } \\
\text { pauvres }\end{array}$ & $\begin{array}{l}\text { Huile de } \\
\text { friture }\end{array}$ & Fév. 2008 & Juil. 2008 & -- & 516 \\
\hline
\end{tabular}


Annexe tableau A.6. Mesures à court terme prises : Indonésie (suite)

\begin{tabular}{|c|c|c|c|c|c|c|c|}
\hline \multirow[b]{2}{*}{ Étiquette } & \multirow[b]{2}{*}{ Description de la mesure } & \multirow[b]{2}{*}{ Objectif } & \multirow[b]{2}{*}{ Produit } & \multirow{2}{*}{$\begin{array}{c}\text { Date de } \\
\text { début }\end{array}$} & \multirow[b]{2}{*}{ Date de fin ${ }^{11}$} & \multicolumn{2}{|c|}{$\begin{array}{l}\text { Impact budgétaire } \\
\text { (milliards IDR) }\end{array}$} \\
\hline & & & & & & 2007 & 2008 \\
\hline $\mathrm{C} 2$ & $\begin{array}{l}\text { Développer la distribution de riz } \\
\text { subventionné dans le cadre de Rankin } \\
\text { (programme Riz pour les pauvres). En } \\
2007, \text { le nombre de ménages pouvant en } \\
\text { bénéficier a été porté à } 15.8 \text { millions, } \\
\text { contre } 10.8 \text { millions en } 2006 \text {. En } 2008 \text {, } \\
\text { il a été porté à } 19.1 \text { millions. En outre, la } \\
\text { ration mensuelle de riz a été portée de } \\
10 \text { à } 15 \mathrm{~kg} \text { pour } 9 \text { des } 10 \text { mois, bien que } \\
\text { le prix payé pour le riz ait été relevé de } \\
1000 \text { IDR/kg à } 1600 \text { IDR/kg. Pour } \\
2009, \text { le nombre de ménages pouvant en } \\
\text { bénéficier a été ramené à } 18.5 \text { millions } \\
\text { mais la ration mensuelle était fournie } \\
\text { pour les } 12 \text { mois de l'année, le prix } \\
\text { subventionné demeurant identique. }\end{array}$ & $\begin{array}{l}\text { Atténuer l'impact } \\
\text { de la hausse des } \\
\text { prix sur les plus } \\
\text { pauvres }\end{array}$ & $\mathrm{Riz}$ & Fév. 2008 & $\begin{array}{c}\text { Toujours en } \\
\text { cours }\end{array}$ & 2346 & 4806 \\
\hline $\mathrm{P} 1$ & $\begin{array}{l}\text { Augmentation du prix de référence } \\
\text { d'achat du riz par les pouvoirs } \\
\text { publics (HPP). Par exemple, le prix du } \\
\text { riz paddy a été augmenté de } 15 \% \text { en } \\
2007 \text {, de } 10 \% \text { en } 2008 \text { et de } 5 \% \text { en } \\
2009 .{ }^{10}\end{array}$ & $\begin{array}{l}\text { Compenser } \\
\text { l'augmentation } \\
\text { des coûts de } \\
\text { production et } \\
\text { suivre la hausse } \\
\text { des cours } \\
\text { mondiaux } \\
\end{array}$ & Riz & 2007 & $\begin{array}{c}\text { Toujours en } \\
\text { cours }\end{array}$ & 675 & 2400 \\
\hline $\mathrm{P} 2$ & $\begin{array}{l}\text { Extension du programme d'aide en } \\
\text { matière de semences, qui assure la } \\
\text { distribution de semences aux } \\
\text { exploitants - augmentation de la } \\
\text { quantité de semences fournies aux } \\
\text { riziculteurs et élargissement aux } \\
\text { semences de maïs et de soja }\end{array}$ & $\begin{array}{l}\text { Accroître la } \\
\text { production en } \\
\text { améliorant les } \\
\text { rendements }\end{array}$ & $\begin{array}{l}\text { Riz, maïs, } \\
\text { soja }\end{array}$ & Janv. 2007 & $\begin{array}{c}\text { Toujours en } \\
\text { cours }\end{array}$ & 875 & 3135 \\
\hline $\mathrm{P} 2$ & $\begin{array}{l}\text { Augmentation des subventions aux } \\
\text { engrais. Le prix de détail maximum des } \\
\text { engrais n'a pas évolué depuis } \\
\text { janvier } 2007 \text {. }\end{array}$ & $\begin{array}{l}\text { Accroître la } \\
\text { production }\end{array}$ & Riz & Juin 2008 & $\begin{array}{c}\text { Toujours en } \\
\text { cours }\end{array}$ & 3095 & 12016 \\
\hline
\end{tabular}


«-- » : aucun impact budgétaire ; « n.d. » : non disponible.

1. Afin de protéger les exploitants les droits de douane à l'importation ont été portés de $450 \mathrm{IDR} / \mathrm{kg}$ à $550 \mathrm{IDR} / \mathrm{kg}$ entre septembre 2007 et février 2008, date où ils ont été ramenés à $450 \mathrm{IDR} / \mathrm{kg}$.

2. Le prix de référence est utilisé pour calculer la taxe à l'exportation acquittée et il est déterminé par le prix c.a.f. à Rotterdam. 3. Le 8 janvier 2007, la TVA de $10 \%$ a été supprimée pour un certain nombre de produits agricoles d'importance stratégique dont le maïs, la viande, la volaille, les œufs et le lait frais. Cette mesure visait toutefois bien plus à améliorer et à accroître la compétitivité du secteur plutôt qu'à compenser l'impact de la hausse des prix, aussi n'apparaît-elle pas dans le tableau.

4. Le Bulog ne peut importer de riz que si ses stocks tombent en dessous de 1 million de tonnes ou que le prix de vente au détail sur le marché de riz de qualité moyenne dépasse 3550 IDR/kg (USD 3.90/kg). En 2006, le Bulog a procédé, conformément aux instructions qui lui avaient été données, à l'importation de 320000 tonnes au total en vue de reconstituer ses stocks régulateurs. En 2008, le Bulog a été autorisé à importer 0.57 million de tonnes mais n'a importé que 70000 tonnes du fait de la bonne récolte de riz réalisée cette année.

5. Afin d'assurer une livraison rapide de ce million de tonnes, le Bulog a désigné un petit nombre d'entreprises privées (une dizaine) chargées d'importer $20 \%$ du volume total, et de le distribuer en se conformant aux plafonds de prix officiels. C'était la première fois depuis 2004 que des négociants privés étaient autorisés à s'engager dans l'importation de riz de qualité moyenne. Le 10 janvier 2004, le gouvernement a annoncé une interdiction saisonnière des importations de riz entre janvier et juin - un moins avant et deux mois après la saison où la récolte bat son plein. Cette interdiction a été prorogée à plusieurs reprises, de sorte qu'elle a fini par prendre toutes les apparences d'une interdiction permanente. Les négociants sont autorisés à importer du riz gluant et autres riz spéciaux.

6 . Le 11 avril 2008 , le gouvernement a publié de « nouvelles » exigences en matière d'importation qui sont fondamentalement identiques à celles instaurées en 2004.

7. Ces mesures de restriction des exportations ont été mises en place lorsque les cours mondiaux du riz ont dépassé les prix indonésiens pour la première fois depuis de nombreuses années.

8. Au second semestre 2008, la demande de devises demeurant excessive (en raison du prix élevé des importations de gaz), une nouvelle contraction de l'offre (du fait d'un effondrement des prix des produits de base) et la diminution de ses réserves de devises ont contraint la Banque d'Indonésie à renoncer à cette ligne de conduite et à permettre une dépréciation de la roupie.

9. La date de fin correspond au mois où le taux d'intérêt de la politique monétaire a été réduit afin de stimuler l'économie.

10. Depuis mars 2005, ces montants n'imposent plus un prix plancher à la production, puisque les pouvoirs publics ne sont plus tenus d'acheter des quantités illimitées de riz aux prix en question. Ces prix sont dits "de référence" du fait qu'ils visent à guider le BULOG dans ses activités de commercialisation.

11. La mention « Toujours en cours » indique que la mesure considérée était encore en vigueur au 31 décembre 2009.

Sources:

Banque d'Indonésie (2010), Economic Report of Indonesia, (diverses années), Banque d'Indonésie, Djakarta, www.bi.go.id.

FAO (2010), Suivi du marché du riz, divers numéros, FAO, Rome, www.fao.org/es/ESC/fr/15/70/highlight 71.html.

GAIN-ID7007 (2007), Indonesia: Oilseeds and Products - Annual 2007, USDA/FAS, $1^{\text {er }}$ mars.

GAIN-ID7011 (2007), Indonesia: Grain and Feed - Annual 2007, USDA/FAS, 2 avril.

GAIN-ID8002 (2008), Indonesia: Oilseeds and Products - Annual 2008, USDA/FAS, 19 février.

GAIN-ID8004 (2008), Indonesia: Grain and Feed - Annual 2008, USDA/FAS, $1^{\mathrm{er}}$ avril.

GAIN-ID8012 (2008), Indonesia: Grain and Feed - New regulations on Indonesian rice exports and imports, USDA/FAS, 21 avril.

GAIN-ID9006 (2009), Indonesia: Grain and Feed - Annual 2009, USDA/FAS, 6 avril.

GAIN-ID9013 (2009), Indonesia: Oilseeds and Products - Annual 2009, USDA/FAS, 5 mai.

OMC (2007), Examen des politiques commerciales : Indonésie, WT/TPR/S/184, OMC, Genève, 23 mai,

www.wto.org/french/tratop e/tpr e/tp285 e.htm. 
Annexe tableau A.7. Mesures à court terme prises : Russie

\begin{tabular}{|c|c|c|c|c|c|c|c|}
\hline \multirow[b]{2}{*}{ Étiquette } & \multirow[b]{2}{*}{ Description de la mesure } & \multirow[b]{2}{*}{ Objectif } & \multirow[b]{2}{*}{ Produit } & \multirow{2}{*}{$\begin{array}{c}\text { Date de } \\
\text { début }\end{array}$} & \multirow[b]{2}{*}{ Date de fin? } & \multicolumn{2}{|c|}{$\begin{array}{c}\text { Impact } \\
\text { budgétaire } \\
\text { (millions RUB) }\end{array}$} \\
\hline & & & & & & 2007 & 2008 \\
\hline M1 & $\begin{array}{l}\text { Réduction des droits de douane, ramenés } \\
\text { de } 5 \% \text { à } 0 \% \text { Cette réduction devait } \\
\text { initialement être valable pour une période } \\
\text { de neuf mois s'étendant de septembre à } \\
\text { juin, mais elle a été prorogée pour une } \\
\text { période supplémentaire de neuf mois en } \\
\text { juin } 2008 \text {, puis de nouveau de } 2.5 \text { mois en } \\
\text { avril } 2009 \text {. }\end{array}$ & $\begin{array}{l}\text { Réduire la } \\
\text { pression des } \\
\text { prix }\end{array}$ & $\begin{array}{l}\text { Huiles de } \\
\text { palme, de } \\
\text { coco (copra) } \\
\text { et de } \\
\text { palmiste en } \\
\quad \text { vrac }\end{array}$ & $\begin{array}{l}12 \text { sept. } \\
2007\end{array}$ & 31 mai 2009 & 171 & 1030 \\
\hline M1 & $\begin{array}{l}\text { Réduction des droits de douane, ramenés } \\
\text { de } 15 \% \text { à } 5 \%\end{array}$ & $\begin{array}{l}\text { Réduire la } \\
\text { pression des } \\
\text { prix }\end{array}$ & $\begin{array}{l}\text { Lait et } \\
\text { produits } \\
\text { laitiers }\end{array}$ & $1^{\mathrm{er}}$ nov. 2007 & $\begin{array}{l}30 \text { avril } \\
2008\end{array}$ & 390 & 926 \\
\hline M1 & $\begin{array}{l}\text { Réduction des droits de douane, ramenés } \\
\text { de } 15 \% \text { à } 5 \% \text { Cette réduction devait } \\
\text { initialement être valable pour une période } \\
\text { de six mois allant de décembre à mai, mais } \\
\text { elle a été prorogée au début juin pour sept } \\
\text { mois supplémentaires. }\end{array}$ & $\begin{array}{l}\text { Réduire la } \\
\text { pression des } \\
\text { prix }\end{array}$ & $\begin{array}{l}\text { Huiles de } \\
\text { soja, de } \\
\text { colza et de } \\
\text { tournesol }^{1}\end{array}$ & $1^{\text {er }}$ déc. 2007 & 31 déc. 2008 & 7 & 335 \\
\hline M2 & $\begin{array}{l}\text { Instauration d'une taxe à l'exportation de } \\
10 \% \text {, mais dont le montant ne pouvait être } \\
\text { inférieur à } 22 \text { EUR la tonne, sur les } \\
\text { exportations vers les pays extérieurs à } \\
\text { l'accord d'union douanière. }{ }^{2} \text { Applicable à } \\
\text { partir du } 28 \text { janvier, la taxe à l'exportation } \\
\text { a été portée à } 40 \% \text {, son montant ne } \\
\text { pouvant être inférieur à } 105 \text { EUR/tonne. À } \\
\text { compter du } 18 \text { mars, les exportations à } \\
\text { destination du Bélarus et du Kazakhstan } \\
\text { ont été interdites afin d'éviter le } \\
\text { contournement des droits à l'exportation. } \\
\text { La taxe à l'exportation et la mesure } \\
\text { d'interdiction, qui étaient initialement } \\
\text { supposées prendre fin le } 30 \text { avril } 2008 \text {, ont } \\
\text { été prorogées en mars jusqu'à la fin juin. }{ }^{3}\end{array}$ & $\begin{array}{l}\text { Freiner la } \\
\text { hausse des prix } \\
\text { intérieurs }\end{array}$ & Blé et méteil & 12 nov. 2007 & 30 juin 2008 & -1360 & -2158 \\
\hline M2 & $\begin{array}{l}\text { Instauration d'une taxe à l'exportation de } \\
30 \% \text {, mais dont le montant ne pouvait être } \\
\text { inférieur à } 70 \text { EUR la tonne, sur les } \\
\text { exportations vers les pays extérieurs à } \\
\text { l'accord d'union douanière. }{ }^{2} \text { La taxe à } \\
\text { l'exportation devait initialement prendre } \\
\text { fin le } 30 \text { avril } 2008 \text { mais a été prorogée en } \\
\text { mars jusqu'à la fin juin. }{ }^{3}\end{array}$ & $\begin{array}{l}\text { Freiner la } \\
\text { hausse des prix } \\
\text { intérieurs }\end{array}$ & Orge & 12 nov. 2007 & 30 juin 2008 & -15 & -45 \\
\hline M3 & $\begin{array}{l}\text { Instauration de taux d'intérêt bonifiés sur } \\
\text { les avances de fonds de roulement } \\
\text { accordés aux transformateurs pour l'achat } \\
\text { de matières premières. Les sociétés } \\
\text { sollicitant ces avances subventionnées } \\
\text { doivent accepter de se conformer aux } \\
\text { mesures de contrôle des prix. }\end{array}$ & $\begin{array}{l}\text { Maintenir la } \\
\text { stabilité des } \\
\text { prix à la } \\
\text { consommation }\end{array}$ & $\begin{array}{l}\text { Transformat } \\
\text { eurs de } \\
\text { denrées } \\
\text { alimentaires } \\
\text { de première } \\
\text { nécessité }\end{array}$ & 24 oct. 2007 & $\begin{array}{l}30 \text { avril } \\
2008\end{array}$ & -- & -- \\
\hline M4 & $\begin{array}{l}\text { Les prix des denrées alimentaires de } \\
\text { première nécessité ont été gelés à leur } \\
\text { niveau du } 15 \text { octobre dans un accord } \\
\text { officiel signé le } 24 \text { octobre } 2007 \text { entre le } \\
\text { ministère de l'Agriculture et les principaux } \\
\text { transformateurs et détaillants de denrées } \\
\text { alimentaires. Aux termes de l'accord } \\
\text { initial, les prix de ces produits devaient } \\
\text { être gelés jusqu'au } 31 \text { janvier } 2008 \text {. Un } \\
\text { nouvel accord, signé le } 31 \text { janvier, a } \\
\text { prolongé le gel des prix de trois mois } \\
\text { supplémentaires, bien que le niveau } \\
\text { maximal auquel les prix étaient gelés ait } \\
\text { été relevé de } 10 \%-15 \% \text {. }\end{array}$ & $\begin{array}{l}\text { Maintenir la } \\
\text { stabilité des } \\
\text { prix à la } \\
\text { consommation }\end{array}$ & $\begin{array}{c}\text { Denrées } \\
\text { alimentaires } \\
\text { de première } \\
\text { nécessité }\end{array}$ & $1^{\mathrm{er}}$ oct. 2007 & $\begin{array}{l}30 \text { avril } \\
2008\end{array}$ & -- & -- \\
\hline
\end{tabular}


Annexe tableau A.7. Mesures à court terme prises : Russie (suite)

\begin{tabular}{|c|c|c|c|c|c|c|c|}
\hline \multirow[b]{2}{*}{ Étiquette } & \multirow[b]{2}{*}{ Description de la mesure } & \multirow[b]{2}{*}{ Objectif } & \multirow[b]{2}{*}{ Produit } & \multirow{2}{*}{$\begin{array}{c}\text { Date de } \\
\text { début }\end{array}$} & \multirow[b]{2}{*}{ Date de fin ${ }^{9}$} & \multicolumn{2}{|c|}{$\begin{array}{c}\text { Impact } \\
\text { budgétaire } \\
\text { (millions RUB) }\end{array}$} \\
\hline & & & & & & 2007 & 2008 \\
\hline M5 & $\begin{array}{l}\text { Autorisation d'exporter vers la Russie } \\
\text { donnée à de nouvelles usines laitières } \\
\text { d'Ukraine et du Bélarus }\end{array}$ & $\begin{array}{l}\text { Accroître les } \\
\text { possibilités } \\
\text { d'approvisionne } \\
\text { ment }\end{array}$ & $\begin{array}{l}\text { Lait et } \\
\text { produits } \\
\text { laitiers }\end{array}$ & Mai 2008 & $\begin{array}{c}\text { Toujours en } \\
\text { cours }\end{array}$ & -- & -- \\
\hline M7 & $\begin{array}{l}\text { Déblocage de } 1.3 \text { million de tonnes de } \\
\text { céréales ( } 85 \% \text { du stock d'intervention), } \\
\text { principalement dans les centres industriels } \\
\text { et les régions importatrices de céréales }\end{array}$ & $\begin{array}{l}\text { Freiner la } \\
\text { hausse des prix } \\
\text { intérieurs }\end{array}$ & Céréales & Oct. 2007 & Juin 2008 & & -- \\
\hline M9 & $\begin{array}{l}\text { Résolution ordonnant au Service fédéral } \\
\text { de lutte contre les monopoles de coopérer } \\
\text { avec les dirigeants régionaux pour } \\
\text { s'assurer que les producteurs et les } \\
\text { détaillants de denrées alimentaires } \\
\text { respectent la législation monopolistique, } \\
\text { en prêtant une attention toute particulière } \\
\text { aux produits laitiers }\end{array}$ & $\begin{array}{l}\text { Freiner la } \\
\text { hausse des prix } \\
\text { intérieurs }\end{array}$ & Tous & Nov. 2007 & $\begin{array}{c}\text { Toujours en } \\
\text { cours }\end{array}$ & -- & -- \\
\hline I1 & $\begin{array}{l}\text { La Banque centrale de Russie a relevé ses } \\
\text { taux fixes sur les opérations de dépôt } \\
\text { réalisées avec les institutions de crédit }\end{array}$ & $\begin{array}{l}\text { Réduire la } \\
\text { masse } \\
\text { monétaire }\end{array}$ & Tous & Mars 2006 & Avril 2009 & -- & -- \\
\hline I1 & $\begin{array}{l}\text { La Banque centrale de Russie a relevé } \\
\text { d'environ } 50 \% \text { les taux de réserves } \\
\text { obligatoires par rapport aux exigibilités } \\
\text { des institutions de crédit }{ }^{5}\end{array}$ & $\begin{array}{l}\text { Alléger les } \\
\text { pressions } \\
\text { inflationnistes }\end{array}$ & Tous & Janv. 2008 & Sept. 2008 & -- & -- \\
\hline I1 & $\begin{array}{l}\text { La Banque centrale de Russie a relevé de } \\
300 \text { points de base son taux de } \\
\text { refinancement (porté de } 10 \% \text { à } 13 \% \text { ) entre } \\
\text { février } 2008 \text { et décembre } 2008^{6}\end{array}$ & $\begin{array}{l}\text { Freiner la } \\
\text { demande et } \\
\text { l'inflation }\end{array}$ & Tous & Fév. 2008 & Avril 2009 & -- & -- \\
\hline $\mathrm{P} 1$ & $\begin{array}{l}\text { Versement d'une subvention } \\
\text { exceptionnelle aux producteurs de porc et } \\
\text { de volailles en fonction du poids-vif des } \\
\text { bêtes envoyées à l'abattoir de janvier à } \\
\text { juin } 2008^{7}\end{array}$ & $\begin{array}{l}\text { Compenser la } \\
\text { hausse des } \\
\text { produits } \\
\text { d'alimentation } \\
\text { des animaux }\end{array}$ & $\begin{array}{l}\text { Porc et } \\
\text { volaille }\end{array}$ & Janv. 2008 & Juin 2008 & -- & 10000 \\
\hline $\mathrm{P} 1$ & $\begin{array}{l}\text { Augmentation de } 60 \% \text { des prix d'achat des } \\
\text { céréales en } 2008 / 09 .{ }^{8} \text { Les nouveaux prix } \\
\text { ont été annoncés en mars } 2008 \text {, quatre } \\
\text { mois avant le début de la récolte. Jamais } \\
\text { ils n'ont été annoncés si tôt. }\end{array}$ & $\begin{array}{l}\text { Accroître les } \\
\text { stocks } \\
\text { d'intervention }\end{array}$ & $\begin{array}{l}\text { Blé et } \\
\text { seigle }\end{array}$ & Juil. 2008 & Juin 2009 & -- & 46100 \\
\hline
\end{tabular}

«-- » : aucun impact budgétaire ; « n.d. » : non disponible.

1. La réduction des droits de douane s'applique à tous les types d'huiles de soja et de colza mais dans le cas de l'huile de tournesol elle ne vaut que pour le vrac.

2. Les membres de l'accord d'union douanière sont le Bélarus, le Kazakhstan, le Kirghizistan, l'Ouzbékistan, la Russie et le Tadjikistan.

3. Les recettes budgétaires sont estimées en supposant que la taxe à l'exportation minimum est acquittée sur l'ensemble du volume de produit exporté au cours de la période considérée, à l'exclusion des exportations vers les membres de l'accord d'union douanière. 4. Y compris le pain de blé, le pain de seigle, le lait (teneur en matière grasse égale ou supérieure à 1.5\%), kéfir (teneur en matière grasse égale ou supérieure à $1 \%$ ), huile de tournesol en bouteille et œufs de volaille.

5. Par exemple, le taux de réserve pour les exigibilités à l'égard des banques non résidentes a été porté de $3.5 \%$ à $8.5 \%$.

6. La date de fin correspond au mois où le taux d'intérêt de la politique monétaire a été réduit afin de stimuler l'économie.

7. $10 \mathrm{RUB} / \mathrm{kg}(0.40 \mathrm{USD} / \mathrm{kg})$ pour les porcs et $5 \mathrm{RUB} / \mathrm{kg}(0.20 \mathrm{USD} / \mathrm{kg})$ pour la volaille. Cette mesure a été annoncée le 6 octobre 2008 et la subvention a été versée rétrospectivement.

8. Le gouvernement n'a pas été en mesure d'acheter de céréales pour le fonds d'intervention en $2007 / 08$ car les prix du marché ont atteint des niveaux bien supérieurs aux prix d'achat par les pouvoirs publics fixés pour la récolte de 2007.

9. La mention « Toujours en cours » indique que la mesure considérée était encore en vigueur au 31 décembre 2009. 
Sources:

Banque centrale de Russie (2010), Annual Report, diverses années, BCR, Moscou, www.cbr.ru.

GAIN-RS7033 (2007), Russia: Grain and Feed - Annual 2007, USDA/FAS, 11 avril.

GAIN-RS7056 (2007), Russia: Trade Policy Monitoring - Import duties on tropical oils, USDA/FAS, 9 août.

GAIN-RS7070 (2007), Russia: Grain and Feed - Russian government resolution on temporary export duties on wheat and barley,

USDA/FAS, 16 octobre.

GAIN-RS7071 (2007), Russia: Dairy and Products - Certain dairy import tariffs reduced, USDA/FAS, 18 octobre.

GAIN-RS7091 (2007), Russia: Agricultural Situation - Russia may prolong food price controls as inflation continues to grow,

USDA/FAS, 14 décembre.

GAIN-RS8006 (2008), Russia: Agricultural Situation - Food price control will be extended until May 12008 , USDA/FAS, $1^{\mathrm{er}}$ janvier.

GAIN-RS8038 (2008), Russia: Oilseeds and Products - Annual Soybean Report 2008, USDA/FAS, 22 mai.

GAIN-RS8042 (2008), Russia: Oilseeds and Products - Extensions of low import tariffs on soybean, rapeseed and sunflower seed oils, USDA/FAS, 9 juin.

GAIN-RS8043 (2008), Russia: Trade Policy Monitoring - Extensions of duty-free bulk imports of tropical oils, USDA/FAS, 10 juin.

GAIN-RS8078 (2008), Russia: Agricultural Situation - Feed Subsidies, USDA FAS, 14 octobre.

GAIN-RS9023 (2009), Russia: Grain and Feed - Annual 2009, USDA/FAS, 31 mars.

GAIN-RS9029 (2009), Russia: Temporary duty-free imports of tropical oils, USDA/FAS, 5 avril.

GAIN-RS9037 (2009), Russia: Grain and Feed - Grain procurement interventions completed, USDA/FAS, 6 avril. 
Annexe tableau A.8. Mesures à court terme prises : Afrique du Sud

\begin{tabular}{|c|c|c|c|c|c|c|c|}
\hline \multirow[b]{2}{*}{ Étiquette } & \multirow[b]{2}{*}{ Description de la mesure } & \multirow[b]{2}{*}{ Objectif } & \multirow[b]{2}{*}{ Produit } & \multirow{2}{*}{$\begin{array}{c}\text { Date de } \\
\text { début }\end{array}$} & \multirow[b]{2}{*}{ Date de fin 9} & \multicolumn{2}{|c|}{$\begin{array}{c}\text { Impact budgétaire } \\
\text { (millions ZAR) }\end{array}$} \\
\hline & & & & & & 2007 & 2008 \\
\hline M8 & $\begin{array}{l}\text { Réduction de l'objectif de } \\
\text { diffusion des } \\
\text { biocarburants, ramené de } \\
4.5 \% \text { à } 2 \%^{1}\end{array}$ & $\begin{array}{l}\text { Réduire au minimum les } \\
\text { problèmes de sécurité } \\
\text { alimentaire }\end{array}$ & Tous & Déc. 2007 & $\begin{array}{c}\text { Toujours en } \\
\text { cours }\end{array}$ & -- & -- \\
\hline I1 & $\begin{array}{l}\text { La Banque de réserve sud- } \\
\text { africaine a relevé de } 500 \\
\text { points de base sont taux de } \\
\text { politique monétaire (porté } \\
\text { de } 7 \% \text { à } 12 \% \text { ) de juin } 2006 \\
\text { à juin } 2008^{2}\end{array}$ & $\begin{array}{l}\text { Freiner la demande et } \\
\text { l'inflation }\end{array}$ & Tous & Juin 2006 & Déc. 2008 & -- & -- \\
\hline $\mathrm{C} 1$ & $\begin{array}{l}\text { Augmentation des aides } \\
\text { publiques versées tous les } \\
\text { mois et relèvement du seuil } \\
\text { de revenus } \\
\end{array}$ & $\begin{array}{l}\text { Réduire la charge financière } \\
\text { imposée par la hausse des } \\
\text { prix }\end{array}$ & Tous & Avril 2008 & $\begin{array}{c}\text { Toujours en } \\
\text { cours }\end{array}$ & 5415 & 13573 \\
\hline $\mathrm{C} 1$ & $\begin{array}{l}\text { Augmentation } \\
\text { exceptionnelle de } \\
\text { l'allocation versée au titre } \\
\text { de l'aide sociale aux } \\
\text { personnes en situation de } \\
\text { détresse (Social Relief of } \\
\text { Distress - SRD) } \\
\end{array}$ & $\begin{array}{l}\text { Protéger les pauvres des } \\
\text { difficultés indues générées } \\
\text { par la hausse des prix } \\
\text { alimentaires et par } \\
\text { l'effondrement de l'économie } \\
\text { mondiale }\end{array}$ & Tous & Avril 2008 & Mars 2009 & -- & 500 \\
\hline $\mathrm{C} 2$ & $\begin{array}{l}\text { Augmentation des } \\
\text { dépenses budgétaires au } \\
\text { titre du programme de } \\
\text { repas scolaires }\end{array}$ & $\begin{array}{l}\text { Tenter de garantir que les } \\
\text { élèves les plus pauvres aient } \\
\text { au moins un bon repas par } \\
\text { jour à l'école }\end{array}$ & Tous & Avril 2007 & $\begin{array}{c}\text { Toujours en } \\
\text { cours }\end{array}$ & 121 & 829 \\
\hline $\mathrm{C} 2$ & $\begin{array}{l}\text { Création de banques } \\
\text { alimentaires d'initiative } \\
\text { locale }^{6}\end{array}$ & $\begin{array}{l}\text { Améliorer la sécurité } \\
\text { alimentaire de la population } \\
\text { locale en lui permettant } \\
\text { d'avoir accès à une } \\
\text { alimentation nutritive et en } \\
\text { quantité suffisante }\end{array}$ & Tous & Avril 2008 & $\begin{array}{c}\text { Toujours en } \\
\text { cours }\end{array}$ & -- & n.d. \\
\hline P2 & $\begin{array}{l}\text { Développement du } \\
\text { programme de production } \\
\text { alimentaire des ménages } \\
\text { (Household Food } \\
\text { Production Programme- } \\
\text { HFPP) }\end{array}$ & $\begin{array}{l}\text { Accroître de } 70000 \text { unités le } \\
\text { nombre de ménages } \\
\text { bénéficiaires des « kits de } \\
\text { démarrage » }\end{array}$ & Tous & Avril 2008 & Mars 2009 & -- & 76 \\
\hline $\mathrm{P} 3$ et $\mathrm{P} 4$ & $\begin{array}{l}\text { Lancement de la campagne } \\
\text { Ilima/Letzema }^{8}\end{array}$ & $\begin{array}{l}\text { Accroître la production } \\
\text { alimentaire en exploitant } \\
\text { toutes les terres productives }\end{array}$ & Tous & Nov. 2008 & $\begin{array}{c}\text { Toujours en } \\
\text { cours }\end{array}$ & -- & 96 \\
\hline
\end{tabular}


«-- » : aucun impact budgétaire ; «n.d. » : non disponible.

1. Certaines différences peuvent être observées entre le projet de stratégie adopté en décembre 2006 aux fins de consultation du public et la stratégie finale rendue publique en décembre 2007 . On estime que le niveau de $2 \%$ exigera environ $1.4 \%$ des terres arables en Afrique du Sud et peut être atteint sans compromettre la sécurité alimentaire.

2. La date de fin correspond au mois où le taux d'intérêt de la politique monétaire a été réduit afin de stimuler l'économie.

3. Les seuils de revenu ont été majorés pour permettre aux personnes ayant des ressources légèrement plus élevées de bénéficier d'une aide. Par exemple, en août 2008, le seuil de revenus pour bénéficier de l'aide au titre des enfants à charge, qui n'avait pas été modifié depuis l'instauration de celle-ci en 1988, a été relevé -il a en fait été multiplié par deux pour tenir compte de l'inflation. Au lieu d'établir un nouveau seuil statique, les autorités ont préféré adopter une formule qui en fixe le niveau à 10 fois le montant de l'aide.

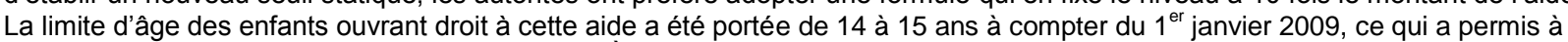
220000 enfants supplémentaires d'en bénéficier. À l'autre extrémité, l'âge à partir duquel les hommes peuvent bénéficier d'une pension de vieillesse est en passe d'être ramené de 65 à 60 ans - c'est-à-dire au même âge que pour les femmes - sur la période 2008-10.

4. Le SRD est un programme fournissant une aide temporaire (3 mois au maximum) sous la forme d'un montant en espèces, de bons d'alimentation ou de colis alimentaires destinés aux personnes en situation d'extrême nécessité qui ne peuvent satisfaire les besoins les plus fondamentaux de leurs familles. II est destiné à faire face à d'extrêmes difficultés, et il fournit une réponse immédiate à une situation de crise lorsque les intéressés n'ont pas les moyens de s'en sortir par eux-mêmes.

5. Les dépenses ont été accrues pour permettre à tous les enfants, dans toutes les écoles couvertes par le programme, de bénéficier d'au moins un bon repas par jour. À compter d'avril 2009, le NSNP a été étendu de manière à couvrir plus de 1500 établissements d'enseignement secondaire considérés comme les plus pauvres parmi les pauvres (premier ou second quintile de la population). 6. Au cours de cette période, le ministère du Développement social a soutenu quatre banques alimentaires d'initiative locale à Durban, Port Elizabeth et Johannesburg, et une banque alimentaire pilote dans un village rural à Umkhanyakude. Le ministère a facilité la signature d'un protocole d'accord entre le gouvernement sud-africain, le réseau des banques alimentaires d'initiative locale d'Afrique du Sud, et le réseau mondial de banques alimentaires.

7. Le HFPP fournit des semis, des semences, des engrais, des pesticides et d'autres intrants de production aux ménages vulnérables. Au total, 79866 ménages vulnérables et groupes d'exploitants en herbe ont été soutenus au moyen de « kits de démarrage » dans le cadre du HFPP en 2008/09 - contre seulement 15765 en 2007/08.

8. La campagne a été lancée par le ministère de l'Agriculture dans huit provinces (toutes à l'exception de celle du Nord-Ouest, et elle a coïncidé avec l'été, saison des semailles. Par rapport au HFPP, qui vise à augmenter la production alimentaire des ménages, le programme llima/Letsema est axé sur la production alimentaire à un niveau plus général en mobilisant les populations locales et en les incitant à tirer parti des terres sous-exploitées (et en particulier les jachères) au travers de programmes de remise en état des terres et d'irrigation, et en encourager les exploitants en herbe à améliorer leur production agricole.

9. La mention « Toujours en cours » indique que la mesure considérée était encore en vigueur au 31 décembre 2009.

\section{Sources:}

Ministère de l'Agriculture [DOA] (2008), Annual Report, diverses années, DSD, Pretoria, www.info.gov.za.

DOA (2009), Agriculture Strategic Plan 2009/10, DOA, Pretoria. www.info.gov.za.

Ministère des minerais et de l'énergie (2007), Biofuels Industrial Strategy, www.dme.gov.za/energy/renew bio.stm.

Ministère du Développement social [DSD] (2009), Annual Report 2008/2009, DSD, Pretoria,

Kelembe, S. (2009), "Trends in selected food prices and policy responses", communication présentée au Forum mondial sur l'agriculture de l'OCDE de 2009, www.oecd.org/agriculture/globalforum/june09.

Conseil national de commercialisation agricole [National Agricultural Marketing Council - NAMC] (2007), The South African Food Cost Review: 2007, NAMC et ministère de l'Agriculture, www.namc.co.za/ASSETS/Newtradeflow100209/Food\%20Cost\%20Review\%202007.pdf.

Trésor national (2009), Estimates of National Expenditure, Trésor national, Pretoria, diverses années, www.info.gov.za. Trésor national (2009), A people's guide...Budget 2009, Trésor national, Pretoria, www.info.gov.za.

Nhlapo-Hlope, J. (2008), Food price increases: Is a practical, comprehensive government response possible, Knowledge

Management Africa Conference 2009, Dakar, http://isivivane.com/kmafrica/?q=group.economic.challenges.food.price.increases. Banque de réserve de l'Afrique du Sud [Reserve Bank of South Africa - RBSA] (2008), Annual Report, diverses années, RBSA, Pretoria, www.reservebank.co.za. 
Annexe tableau A.9. Mesures à court terme prises : Ukraine

\begin{tabular}{|c|c|c|c|c|c|c|c|}
\hline \multirow[b]{2}{*}{ Étiquette } & \multirow[b]{2}{*}{ Description de la mesure } & \multirow[b]{2}{*}{ Objectif } & \multirow[b]{2}{*}{ Produit } & \multirow{2}{*}{$\begin{array}{c}\text { Date de } \\
\text { début }\end{array}$} & \multirow[b]{2}{*}{ Date de fin ${ }^{7}$} & \multicolumn{2}{|c|}{$\begin{array}{c}\text { Impact } \\
\text { budgétaire } \\
\text { (millions UAH) }\end{array}$} \\
\hline & & & & & & 2007 & 2008 \\
\hline M4 & $\begin{array}{l}\text { Une résolution du conseil des } \\
\text { ministres a conféré aux } \\
\text { administrations locales le pouvoir de } \\
\text { fixer des marges de bénéfices d'au } \\
\text { plus } 10 \% \text { pour les producteurs de } \\
\text { farine de blé et de seigle et pour les } \\
\text { boulangers utilisant ces produits, et de } \\
\text { fixer le prix de gros de ces farines. } \\
\text { En avril } 2008 \text { la marge de gros } \\
\text { maximum a été portée à } 15 \% \text { et } \\
\text { l'éventail de produits pour lesquels } \\
\text { des limites peuvent être fixés a été } \\
\text { élargi. }\end{array}$ & $\begin{array}{l}\text { Limiter les } \\
\text { hausses de prix }\end{array}$ & $\begin{array}{l}\text { Farine et pain } \\
\text { de blé et de } \\
\text { seigle, et } \\
\text { denrées } \\
\text { alimentaires } \\
\text { socialement } \\
\text { importantes }^{2}\end{array}$ & Juil. 2007 & Déc. 2008 & -- & - \\
\hline M4 & $\begin{array}{l}\text { Un protocole d'accord a été signé } \\
\text { entre le gouvernement et les entités } \\
\text { nationales du réseau de vente au détail } \\
\text { en vue de limiter à } 10 \% \text { du prix de } \\
\text { gros le taux de marge pour les } \\
\text { denrées alimentaires socialement } \\
\text { importantes }\end{array}$ & $\begin{array}{l}\text { Fournir les } \\
\text { denrées } \\
\text { alimentaires } \\
\text { socialement } \\
\text { importantes à des } \\
\text { prix abordables }\end{array}$ & $\begin{array}{l}\text { Denrées } \\
\text { alimentaires } \\
\text { socialement } \\
\text { importantes }^{2}\end{array}$ & Avr. 2008 & Déc. 2008 & -- & - \\
\hline M6 & $\begin{array}{l}\text { Contingents à l'exportation } \\
\text { représentant au total } 403000 \text { tonnes } \\
\text { pour la campagne de } \\
\text { commercialisation de } 2006 / 07 \text { et } \\
1203000 \text { tonnes pour celle de } \\
2007 / 08\end{array}$ & $\begin{array}{l}\text { Assurer une offre } \\
\text { de denrées } \\
\text { alimentaires } \\
\text { suffisante et } \\
\text { limiter la hausse } \\
\text { du prix du pain }\end{array}$ & Blé & $\begin{array}{l}17 \text { oct. } \\
2006\end{array}$ & 23 mai 2008 & -- & - \\
\hline M6 & $\begin{array}{l}\text { Contingents à l'exportation } \\
\text { représentant au total } 6000 \text { tonnes } \\
\text { pour la campagne de } \\
\text { commercialisation de } 2006 / 07 \text { et } \\
6000 \text { tonnes pour celle de } 2007 / 08\end{array}$ & $\begin{array}{l}\text { Assurer une offre } \\
\text { de denrées } \\
\text { alimentaires } \\
\text { suffisante et } \\
\text { limiter la hausse } \\
\text { du prix du pain }\end{array}$ & Seigle & $\begin{array}{c}17 \text { oct. } \\
2006\end{array}$ & 23 mai 2008 & -- & - \\
\hline M6 & $\begin{array}{l}\text { Contingents à l'exportation } \\
\text { représentant au total } 1 \text { million de } \\
\text { tonnes pour la campagne de } \\
\text { commercialisation de } 2006 / 07 \text { et } \\
603000 \text { tonnes pour celle de } 2007 / 08\end{array}$ & $\begin{array}{l}\text { Assurer une offre } \\
\text { suffisante et } \\
\text { limiter la hausse } \\
\text { des prix des } \\
\text { produits } \\
\text { d'alimentation des } \\
\text { animaux }\end{array}$ & Maïs & $\begin{array}{c}17 \text { oct. } \\
2006\end{array}$ & $\begin{array}{l}31 \text { mars } \\
2008\end{array}$ & -- & \\
\hline M6 & $\begin{array}{l}\text { Contingents à l'exportation } \\
\text { représentant au total } 1.2 \text { million de } \\
\text { tonnes pour la campagne de } \\
\text { commercialisation de } 2006 / 07 \text { et } \\
903000 \text { tonnes pour celle de } 2007 / 08\end{array}$ & $\begin{array}{l}\text { Assurer une offre } \\
\text { suffisante et } \\
\text { limiter la hausse } \\
\text { des prix des } \\
\text { produits } \\
\text { d'alimentation des } \\
\text { animaux }\end{array}$ & Orge & $\begin{array}{l}17 \text { oct. } \\
2006\end{array}$ & 23 mai 2008 & -- & - \\
\hline M6 & $\begin{array}{l}\text { Contingent d'exportation de } \\
300000 \text { tonnes, portées à } 500000 \text { le } \\
23 \text { avril }\end{array}$ & $\begin{array}{l}\text { Réduire la } \\
\text { pression des prix }\end{array}$ & $\begin{array}{l}\text { Huile de } \\
\text { tournesol }\end{array}$ & $\begin{array}{l}22 \text { mars } \\
2008\end{array}$ & 28 mai 2008 & -- & - \\
\hline M6 & $\begin{array}{l}\text { Contingent d'exportation de } \\
1000 \text { tonnes }\end{array}$ & $\begin{array}{l}\text { Réduire la } \\
\text { pression des prix }\end{array}$ & $\begin{array}{c}\text { Graines de } \\
\text { tournesol }\end{array}$ & $\begin{array}{c}22 \text { mars } \\
2008\end{array}$ & 28 mai 2008 & -- & - \\
\hline M7 & $\begin{array}{l}\text { Le Fonds agraire et le Comité d'Etat } \\
\text { pour les réserves de matières } \\
\text { premières (Derzhkomrezerv) ont mis } \\
\text { sur le marché de la farine produite à } \\
\text { partir de blé acheté lors des récoltes } \\
\text { précédentes : } 375000 \text { tonnes en } 2007 \\
\text { et } 85500 \text { tonnes en } 2008^{4}\end{array}$ & $\begin{array}{l}\text { Éviter les hausses } \\
\text { de prix du blé }\end{array}$ & Farine de blé & Juil. 2007 & Juil. 2008 & -- & - \\
\hline
\end{tabular}


Annexe tableau A.9. Mesures à court terme prises : Ukraine (suite)

\begin{tabular}{|c|c|c|c|c|c|c|c|}
\hline \multirow[b]{2}{*}{ Étiquette } & \multirow[b]{2}{*}{ Description de la mesure } & \multirow[b]{2}{*}{ Objectif } & \multirow[b]{2}{*}{ Produit } & \multirow{2}{*}{$\begin{array}{c}\text { Date de } \\
\text { début }\end{array}$} & \multirow[b]{2}{*}{ Date de fin ${ }^{7}$} & \multicolumn{2}{|c|}{$\begin{array}{c}\text { Impact } \\
\text { budgétaire } \\
\text { (millions UAH) }\end{array}$} \\
\hline & & & & & & 2007 & 2008 \\
\hline M7 & $\begin{array}{l}\text { Le Derzhkomrezerv a vendu à } \\
\text { l'industrie de transformation de } \\
\text { viande } 14600 \text { tonnes de produits issus } \\
\text { de la réserve d'État de matières } \\
\text { premières, ainsi qu'une certaine } \\
\text { quantité de volailles importées aux } \\
\text { magasins de vente au détail }\end{array}$ & $\begin{array}{l}\text { Réduire les prix } \\
\text { de détail, dont le } \\
\text { niveau était élevé }\end{array}$ & Viande & Sept. 2008 & Juil. 2008 & -- & -- \\
\hline M7 & $\begin{array}{l}\text { Le Fonds agraire et le } \\
\text { Derzhkomrezerv ont vendu } \\
2000 \text { tonnes de sucre issu de leurs } \\
\text { réserves }\end{array}$ & $\begin{array}{l}\text { Réduire la } \\
\text { pression des prix }\end{array}$ & Sucre & Janv. 2008 & Juil. 2008 & -- & -- \\
\hline M9 & $\begin{array}{l}\text { Établir des procédures pour notifier } \\
\text { les changements des prix de gros }\end{array}$ & $\begin{array}{l}\text { Assurer une } \\
\text { formation } \\
\text { transparente des } \\
\text { prix }\end{array}$ & $\begin{array}{c}\text { Denrées } \\
\text { alimentaires } \\
\text { socialement } \\
\text { importantes }^{2}\end{array}$ & Déc. 2007 & $\begin{array}{c}\text { Toujours en } \\
\text { cours }\end{array}$ & -- & -- \\
\hline M9 & $\begin{array}{l}\text { Intensification de l'examen de } \\
\text { conformité aux réglementations des } \\
\text { prix par l'Inspection d'État chargée du } \\
\text { contrôle des prix }{ }^{4}\end{array}$ & $\begin{array}{l}\text { Assurer le respect } \\
\text { des } \\
\text { réglementations }\end{array}$ & $\begin{array}{c}\text { Denrées } \\
\text { alimentaires } \\
\text { socialement } \\
\text { importantes }^{2} \\
\end{array}$ & Janv. 2008 & $\begin{array}{l}\text { Toujours en } \\
\text { cours }\end{array}$ & -- & -- \\
\hline I1 & $\begin{array}{l}\text { La Banque nationale } \\
\text { d'Ukraine (BNU) a relevé le taux de } \\
\text { réserves obligatoires en tenant } \\
\text { désormais compte des emprunts en } \\
\text { devise contracté par les banques } \\
\text { locales auprès des institutions } \\
\text { financières étrangères }\end{array}$ & $\begin{array}{l}\text { Limiter les } \\
\text { pressions } \\
\text { inflationnistes }\end{array}$ & Tous & 20 nov. 2007 & $\begin{array}{c}\text { Toujours en } \\
\text { cours }\end{array}$ & -- & -- \\
\hline I1 & $\begin{array}{l}\text { La BNU a relevé de } 400 \text { points de } \\
\text { base (de } 8 \% \text { à } 12 \% \text { ) son taux d'intérêt } \\
\text { de référence entre janvier } 2008 \text { et } \\
\text { avril } 2008^{5}\end{array}$ & $\begin{array}{l}\text { Limiter les } \\
\text { pressions } \\
\text { inflationnistes }\end{array}$ & Tous & Janv. 2008 & Juin 2009 & -- & -- \\
\hline I1 & $\begin{array}{l}\text { La BNU a révalué le taux de change } \\
\text { officiel de la hryvnia par rapport au } \\
\text { dollar des États-Unis, le faisant passer } \\
\text { de } 5.05 \text { UAH à } 4.85 \text { UAH pour } \\
1 \text { USD }^{6}\end{array}$ & $\begin{array}{l}\text { Limiter les } \\
\text { pressions } \\
\text { inflationnistes }\end{array}$ & Tous & 22 mai 2008 & $\begin{array}{l}30 \text { sept. } \\
2008\end{array}$ & -- & -- \\
\hline P1 & $\begin{array}{l}\text { Relèvement du prix minimum } \\
\text { applicable aux achats d'intervention } \\
\text { du Fonds agraire. Par exemple, le prix } \\
\text { minimum du blé de meunerie } \\
\text { ( } 3^{\text {ème }} \text { catégorie), principal produit } \\
\text { acheté, a été porté de } 690 \text { UAH la } \\
\text { tonne lors de son instauration en } \\
2005 / 06 \text { à } 1251 \text { UAH en } 2008 / 09 \text {. }\end{array}$ & $\begin{array}{l}\text { Permettre au } \\
\text { Fonds agraire } \\
\text { d'acheter des } \\
\text { stocks }\end{array}$ & Blé et seigle & 2007 & $\begin{array}{l}\text { Toujours en } \\
\text { cours }\end{array}$ & 401 & 1274 \\
\hline
\end{tabular}

«-- » : aucun impact budgétaire ; « n.d. » : non disponible.

1. Les accords varient selon les oblasts et d'une ville à l'autre. Dans la pratique, une marge de bénéfices de $5 \%$ était souvent utilisée. Les administrations locales fournissaient des subventions aux boulangeries pour garantir qu'elles puissent acheter de la farine en début de saison.

2. Farine, pain, céréales, porc, bœuf, volailles, œufs, lait, crème sûre, beurre, sucre et huile de tournesol.

3. Dans le même temps, le système en place de cartes de réduction pour les denrées alimentaires socialement importantes a été suspendu dans les chaînes de distribution au détail concernées.

4. Au cours du premier semestre 2008, 8900 inspections ont été réalisées par les organismes de contrôle des prix. Des infractions ont été constatées chez $57 \%$ des entités inspectées.

5. La date de fin correspond au mois où le taux d'intérêt de la politique monétaire a été réduit afin de stimuler l'économie.

6. Le $1^{\text {er }}$ octobre 2008, la BNU a dévalué le taux de change officiel, ramené de 4.85 UAH à 7.70 UAH pour 1 USD.

7. La mention « Toujours en cours » indique que la mesure considérée était encore en vigueur au 31 décembre 2009. 
Sources:

Cramon, S. von et M. Raiser (2006), The quotas on grain exports in Ukraine: Ineffective, inefficient and non-transparent, Institute for Economic Research and Policy Consulting, Kiev, http://pdc.ceu.hu/archive/00004700/01/agp10 en.pdf.

GAIN-UP7007 (2007), Ukraine: Grain and Feed - Annual Report 2007, USDA/FAS, 29 mars.

GAIN-UP7009 (2007), Ukraine: Oilseeds and Products - Annual Report 2007, USDA/FAS, 25 avril.

GAIN-UP8005 (2008), Ukraine: Grain and Feed - Annual Report 2008, USDA/FAS, 26 mars.

GAIN-UP8007 (2008), Ukraine: Oilseeds and Products - Annual Report 2008, USDA/FAS, 25 avril.

GAIN-UP9008 (2009), Ukraine: Grain and Feed - Annual Report 2009, USDA/FAS, 26 mars.

GAIN-UP9011 (2009), Ukraine: Oilseeds and Products - Annual Report 2009, USDA/FAS, 30 mars.

Institute for Economic Research and Policy Consulting [IERPC] (2007), "Weather and policy risks - Are the grain export restrictions unavoidable to achieve food security in Ukraine?", Policy Paper $n^{\circ} 14$, IERPC, Kiev,

http://pdc.ceu.hu/archive/00005127/01/agpp14 en.pdf.

Kobouta, Iryna (2008), “Ukraine: Agricultural Policy Development in 2006-2008”, rapport soumis à l'OCDE.

Bank nationale d'Ukraine (2010), Annual Report, diverses années, BNU, Kiev, www.bank.gov.ua/ENGL/DEFAULT.HTM.

Banque mondiale (2008), "Competitive Agriculture or State Control: Ukraine's response to the global food crisis", Report $n^{\circ} 44984-$

UA, Banque mondiale, Washington DC,

http://siteresources.worldbank.org/INTUKRAINE/Resources/WorldFoodCrisisandRoleofUkraine.pdf. 
Annexe tableau A.10. Mesures à court terme prises : Vietnam

\begin{tabular}{|c|c|c|c|c|c|c|c|}
\hline \multirow[b]{2}{*}{ Étiquette } & \multirow[b]{2}{*}{ Description de la mesure } & \multirow[b]{2}{*}{ Objectif } & \multirow[b]{2}{*}{ Produit } & \multirow{2}{*}{$\begin{array}{c}\begin{array}{c}\text { Date de } \\
\text { début }\end{array} \\
\end{array}$} & \multirow[b]{2}{*}{ Date de fin 6} & \multicolumn{2}{|c|}{$\begin{array}{c}\text { Impact } \\
\text { budgétaire } \\
\text { (milliards VND) }\end{array}$} \\
\hline & & & & & & 2007 & 2008 \\
\hline M1 & $\begin{array}{l}\text { Réduction de } 30 \%-50 \% \text { des tarifs } \\
\text { NPF pour un large éventail de } \\
\text { produits. La réduction initiale en août } \\
\text { a été suivie de nouvelles réductions en } \\
\text { octobre } 2007 \text { et en août } 2008 \text { pour } \\
\text { certains nouveaux produits et pour } \\
\text { quelques-uns de ceux ayant déjà } \\
\text { bénéficié d'une réduction. Ces } \\
\text { réductions tarifaires demeurent certes } \\
\text { valables pour la plupart des produits, } \\
\text { mais les droits de douane ont de } \\
\text { nouveau été relevés pour la viande et } \\
\text { pour certains produits laitiers. }\end{array}$ & $\begin{array}{l}\text { Ralentir la } \\
\text { hausse des } \\
\text { prix du } \\
\text { marché }\end{array}$ & $\begin{array}{l}\text { Un large } \\
\text { éventail de } \\
\text { produits }^{1}\end{array}$ & 3 août 2007 & $\begin{array}{c}\text { Toujours en } \\
\text { cours }\end{array}$ & 628 & 2367 \\
\hline M2 & $\begin{array}{l}\text { Instauration d'une taxe à l'exportation } \\
\text { dont le montant augmentait } \\
\text { proportionnellement au prix à } \\
\text { l'exportation au-delà du seuil de } \\
600 \text { USD par tonne. Le } 15 \text { août, le } \\
\text { seuil minimum a été relevé à } \\
800 \text { USD la tonne. }\end{array}$ & $\begin{array}{l}\text { Maintenir } \\
\text { l'approvision } \\
\text { nement } \\
\text { intérieur et } \\
\text { stabiliser les } \\
\text { prix du } \\
\text { marché } \\
\end{array}$ & Riz & 21 juil. 2008 & 19 déc. 2008 & -- & n.d. ${ }^{2}$ \\
\hline M6 & $\begin{array}{l}\text { Interdiction des exportations, sauf } \\
\text { pour les contrats signés avec Cuba et } \\
\text { pour l'Indonésie, pays dont les navires } \\
\text { mouillaient au Vietnam avant le } \\
11 \text { novembre } 2006 \text {. Peu après, } \\
\text { l'objectif d'exportation pour } 2006 \text { a } \\
\text { été ramené de } 5 \text { millions de tonnes à } \\
4.7 \text { millions de tonnes pour tenir } \\
\text { compte du volume des contrats signés } \\
\text { pour cette année. }\end{array}$ & $\begin{array}{l}\text { Maintenir } \\
\text { l'approvision } \\
\text { nement } \\
\text { intérieur et } \\
\text { stabiliser les } \\
\text { prix du } \\
\text { marché }\end{array}$ & Riz & 12 nov. 2006 & Fév. 2007 & -- & -- \\
\hline M6 & $\begin{array}{l}\text { Autorisation de la reprise des es } \\
\text { ventes à l'exportation. L'objectif } \\
\text { d'exportation pour } 2007 \text { a été ramené } \\
\text { à to } 4.5 \text { millions de tonnes, contre } \\
4.7 \text { millions de tonnes en décembre } \\
2006 \text { et un niveau initial de } 5 \text { millions } \\
\text { de tonnes. }\end{array}$ & $\begin{array}{l}\text { Maintenir } \\
\text { l'approvision } \\
\text { nement } \\
\text { intérieur et } \\
\text { stabiliser les } \\
\text { prix du } \\
\text { marché } \\
\end{array}$ & Riz & Fév. 2007 & Déc. 2007 & -- & -- \\
\hline M6 & $\begin{array}{l}\text { Relèvement de } 10 \text { USD par tonne des } \\
\text { prix minimum à l'exportation (PME) } \\
\text { pour toutes les qualités de riz. Les } \\
\text { PME pour le riz contenant de } 5 \% \text { et } \\
25 \% \text { de brisures ont par exemple été } \\
\text { portés à } 300 \text { USD et } 280 \text { USD la } \\
\text { tonne respectivement. Les PME ont } \\
\text { été augmentés tout au long de l'année. }\end{array}$ & $\begin{array}{l}\text { Maintenir } \\
\text { l'approvision } \\
\text { nement } \\
\text { intérieur et } \\
\text { stabiliser les } \\
\text { prix du } \\
\text { marché }\end{array}$ & Riz & Fév. 2007 & Déc. 2007 & -- & -- \\
\hline M6 & $\begin{array}{l}\text { Suspension de l'enregistrement de } \\
\text { nouveaux contrats d'exportation } \\
\text { lorsque le volume total des contrats } \\
\text { passés a atteint l'objectif } \\
\text { d'exportation de } 4.5 \text { millions de } \\
\text { tonnes. Les envois en exécution des } \\
\text { contrats déjà enregistrés étaient } \\
\text { maintenus. Cependant, Vinafood } 2 \text { et } \\
\text { certains exportateurs provinciaux de } \\
\text { denrées alimentaires ont été autorisés } \\
\text { à participer aux appels d'offres de } \\
\text { l'Autorité nationale des Philippines } \\
\text { chargée des approvisionnements } \\
\text { alimentaires publiés en } \\
\text { décembre } 2007 \text { et en janvier } 2008 \text {, } \\
\text { débouchant sur des contrats prévoyant } \\
\text { l'exportation de } 700000 \text { tonnes au } \\
\text { total en } 2008 \text {. }\end{array}$ & $\begin{array}{l}\text { Maintenir } \\
\text { l'approvision } \\
\text { nement } \\
\text { intérieur et } \\
\text { stabiliser les } \\
\text { prix du } \\
\text { marché }\end{array}$ & Riz & 21 juil. 2007 & $\begin{array}{c}18 \text { janv. } \\
2008\end{array}$ & -- & -- \\
\hline
\end{tabular}


Annexe tableau A.10. Mesures à court terme prises : Vietnam (suite)

\begin{tabular}{|c|c|c|c|c|c|c|c|}
\hline \multirow[b]{2}{*}{ Étiquette } & \multirow[b]{2}{*}{ Description de la mesure } & \multirow[b]{2}{*}{ Objectif } & \multirow[b]{2}{*}{ Produit } & \multirow{2}{*}{$\begin{array}{c}\text { Date de } \\
\text { début }\end{array}$} & \multirow[b]{2}{*}{ Date de fin ${ }^{6}$} & \multicolumn{2}{|c|}{$\begin{array}{c}\text { Impact } \\
\text { budgétaire } \\
\text { (milliards VND) }\end{array}$} \\
\hline & & & & & & 2007 & 2008 \\
\hline M6 & $\begin{array}{l}\text { L'enregistrement des contrats } \\
\text { d'exportation a été autorisé à } \\
\text { reprendre, l'objectif d'exportation } \\
\text { pour } 2008 \text { étant fixé à } 4.5 \text { millions de } \\
\text { tonnes, au lieu des } 4.8 \text { millions de } \\
\text { tonnes estimés en septembre } 2007 \text {. } \\
\text { Cependant, la Vietnam Food } \\
\text { Association (VFA) demande } \\
\text { officieusement qu'aucune vente de riz } \\
\text { contenant } 25 \% \text { de brisures ne soit } \\
\text { effectuée - cette qualité de riz } \\
\text { représente environ } 40 \% \text { du volume } \\
\text { des exportations. }\end{array}$ & $\begin{array}{l}\text { Maintenir } \\
\text { l'approvision } \\
\text { nement } \\
\text { intérieur et } \\
\text { stabiliser les } \\
\text { prix du } \\
\text { marché }\end{array}$ & Riz & $\begin{array}{l}18 \text { janv. } \\
2008\end{array}$ & 5 fév. 2008 & -- & -- \\
\hline M6 & $\begin{array}{l}\text { Relèvement des PME pour toutes les } \\
\text { qualités de riz. Les PME pour le riz } \\
\text { contenant } 5 \% \text { et } 25 \% \text { de brisures ont } \\
\text { par exemple été respectivement fixés } \\
\text { à } 385 \text { USD et } 360 \text { USD la tonne pour } \\
\text { les exportations de janvier-février. }\end{array}$ & $\begin{array}{l}\text { Maintenir } \\
\text { l'approvision } \\
\text { nement } \\
\text { intérieur et } \\
\text { stabiliser les } \\
\text { prix du } \\
\text { marché }\end{array}$ & Riz & $\begin{array}{l}18 \text { janv. } \\
2008\end{array}$ & 5 fév. 2008 & -- & -- \\
\hline M6 & $\begin{array}{l}\text { Abrogation des PME, d'où } \\
\text { l'impossibilité pour les négociants } \\
\text { privés de conclure de nouveaux } \\
\text { contrats. Les envois en exécution des } \\
\text { contrats déjà enregistrés ont été } \\
\text { maintenus, tandis que Vinafood } 2 \text { a } \\
\text { continué de participer aux appels } \\
\text { d'offres. Le } 14 \text { mars, la Vietnam Food } \\
\text { Association (VFA) a annoncé qu'elle } \\
\text { interdirait tout nouveau contrat } \\
\text { d'exportation jusqu'à la fin avril. Le } \\
28 \text { avril, cette interdiction a été } \\
\text { prorogée jusqu'en juin. }\end{array}$ & $\begin{array}{l}\text { Assurer des } \\
\text { approvisionne } \\
\text { ments pour } \\
\text { les contrats } \\
\text { avalisés par le } \\
\text { gouvernement }\end{array}$ & Riz & 5 fév. 2008 & 18 juin 2008 & -- & -- \\
\hline M6 & $\begin{array}{l}\text { La VFA a publié de nouvelles } \\
\text { réglementations plus strictes } \\
\text { concernant les exportations de riz. Les } \\
\text { exportateurs doivent détenir au moins } \\
50 \% \text { du volume objet du contrat dans } \\
\text { leurs stocks disponibles, et les prix à } \\
\text { l'exportation devront se conformer } \\
\text { aux indications de prix établies par la } \\
\text { VFA. L'envoi des marchandises devra } \\
\text { avoir lieu dans les deux mois de la } \\
\text { signature du contrat. }\end{array}$ & $\begin{array}{l}\text { Ralentisseme } \\
\text { nt des } \\
\text { exportations }\end{array}$ & Riz & $\begin{array}{l}26 \text { mars } \\
2008\end{array}$ & Déc. 2008 & -- & -- \\
\hline M6 & $\begin{array}{l}\text { L'enregistrement des exportations a } \\
\text { été autorisé à reprendre mais le } \\
\text { volume total était limité à } 3.5 \text { millions } \\
\text { de tonnes pour les neuf mois à courir } \\
\text { jusqu'en septembre, et le PME a en } \\
\text { outre été fixé à } 800 \text { USD la tonne. Ce } \\
\text { montant a été ramené à } 600 \text { USD la } \\
\text { tonne le } 30 \text { juillet puis il a été de } \\
\text { nouveau diminué en septembre. }\end{array}$ & $\begin{array}{l}\text { Maintenir } \\
\text { l'approvision } \\
\text { nement } \\
\text { intérieur et } \\
\text { stabiliser les } \\
\text { prix du } \\
\text { marché }\end{array}$ & Riz & 18 juin 2008 & Déc. 2008 & -- & -- \\
\hline M9 & $\begin{array}{l}\text { Publication de décrets contre les } \\
\text { spéculateurs, interdisant aux non } \\
\text { négociants de faire le commerce de } \\
\text { céréales }\end{array}$ & $\begin{array}{l}\text { Porter un } \\
\text { coup d'arrêt à } \\
\text { la frénésie } \\
\text { d'achats } \\
\text { chaotiques }\end{array}$ & Riz & 28 avr. 2008 & $\begin{array}{c}\text { Toujours en } \\
\text { cours }\end{array}$ & -- & -- \\
\hline M9 & $\begin{array}{l}\text { Création d'une réserve nationale de } \\
\text { riz }\end{array}$ & $\begin{array}{l}\text { Pour lisser les } \\
\text { fluctuations } \\
\text { de l'offre et la } \\
\text { demande }\end{array}$ & Riz & Juin 2008 & $\begin{array}{l}\text { Toujours en } \\
\text { cours }\end{array}$ & -- & 300 \\
\hline
\end{tabular}


Annexe tableau A.10. Mesures à court terme prises : Vietnam (suite)

\begin{tabular}{|c|c|c|c|c|c|c|c|}
\hline \multirow[b]{2}{*}{ Étiquette } & \multirow[b]{2}{*}{ Description de la mesure } & \multirow[b]{2}{*}{ Objectif } & \multirow[b]{2}{*}{ Produit } & \multirow{2}{*}{$\begin{array}{c}\text { Date de } \\
\text { début }\end{array}$} & \multirow[b]{2}{*}{ Date de fin ${ }^{6}$} & \multicolumn{2}{|c|}{$\begin{array}{c}\text { Impact } \\
\text { budgétaire } \\
\text { (milliards VND) }\end{array}$} \\
\hline & & & & & & 2007 & 2008 \\
\hline I1 & $\begin{array}{l}\text { La Banque d'État du Vietnam (State } \\
\text { Bank of Vietnam - SBV) a relevé de } \\
575 \text { points de base (de } 8.25 \% \text { à } 14 \% \text { ) } \\
\text { son taux d'intérêt de référence entre } \\
\text { février } 2008 \text { et juin } 2008^{4}\end{array}$ & $\begin{array}{l}\text { Freiner la } \\
\text { demande et } \\
\text { l'inflation }\end{array}$ & Tous & Fév. 2008 & Oct. 2008 & -- & -- \\
\hline I1 & $\begin{array}{l}\text { La SBV a augmenté le taux de réserve } \\
\text { obligatoires, multiplié par } 1.5-2 \text {. }\end{array}$ & $\begin{array}{l}\text { Maîtriser la } \\
\text { croissance } \\
\text { des liquidités }\end{array}$ & Tous & Juin 2007 & Nov. 2008 & -- & -- \\
\hline $\mathrm{P} 2$ & $\begin{array}{l}\text { Dans le cadre de la réduction générale } \\
\text { des droits de douane, ceux applicables } \\
\text { aux produits d'alimentation animale } \\
\text { ont été abaissés }\end{array}$ & $\begin{array}{l}\text { Réduire les } \\
\text { coûts de } \\
\text { production }\end{array}$ & $\begin{array}{l}\text { Produits } \\
\text { d'alimentatio } \\
\mathrm{n} \text { animale }\end{array}$ & 3 août 2007 & $\begin{array}{l}\text { Toujours en } \\
\text { cours }\end{array}$ & 153 & 701 \\
\hline $\mathrm{P} 2$ & $\begin{array}{l}\text { Exonération de la redevance } \\
\text { d'irrigation pour les ménages ayant } \\
\text { reçu de l'État des espaces terrestres ou } \\
\text { aquatiques pour y pratiquer } \\
\text { l'agriculture, la sylviculture, } \\
\text { l'aquaculture ou la production de sel. }\end{array}$ & $\begin{array}{l}\text { Réduire les } \\
\text { coûts de } \\
\text { production }\end{array}$ & $\begin{array}{l}\text { Cultures } \\
\text { végétales }\end{array}$ & Juil. 2008 & $\begin{array}{l}\text { Toujours en } \\
\text { cours }\end{array}$ & -- & 500 \\
\hline P5 & $\begin{array}{l}\text { Suspension de l'octroi de licences } \\
\text { pour de nouveaux terrains de golf }\end{array}$ & $\begin{array}{l}\text { Maintenir les } \\
\text { terres } \\
\text { disponibles } \\
\text { pour la } \\
\text { production de } \\
\text { riz et protéger } \\
\text { les } \\
\text { agriculteurs } \\
\text { pauvres }\end{array}$ & Riz & Sept. 2008 & $\begin{array}{l}\text { Toujours en } \\
\text { cours }\end{array}$ & -- & -- \\
\hline
\end{tabular}

«-- » : aucun impact budgétaire ; « n.d. » : non disponible.

1. Parmi les plus importantes réductions tarifaires, du point de vue de la perte de recettes consentie par le gouvernement vietnamien, figurent celles dont ont bénéficié la volaille (réduction de $20 \%$ à $12 \%$ ), les poudres de lait (de 10\%-30\% à 5\%-15\%), le maïs (de 5\% à $0 \%$ ) et l'huile de palme (de $30 \%$ à $20 \%$ ).

2. Les recettes budgétaires tirées de la taxe à l'exportation ont sans doute été minimes car les prix à l'exportation sont tombés en dessous du seuil très peu après son instauration. La valeur moyenne mensuelle des exportations de riz du Vietnam est tombée d'environ 970 USD la tonne en juillet à environ 550 USD la tonne en août. Le seuil minimum a été relevé en août en vue de majorer les prix à l'exportation.

3. En outre, les exportateurs peuvent signer des contrats avec tout acheteur sauf l'Autorité nationale chargée des approvisionnements alimentaires aux Philippines, le Bulog en Indonésie, Alimport à Cuba et Bernas en Malaisie. Les contrats portant sur le riz gluant et le riz parfumé ne sont pas soumis à cette restriction.

4. La date de fin correspond au mois où le taux d'intérêt de la politique monétaire a été réduit afin de stimuler l'économie.

5. Les redevances d'irrigation continueront d'être collectées dans les zones où la demande d'eau est supérieure aux possibilités d'approvisionnement, sauf pour les agriculteurs vivant en dessous du seuil de pauvreté. Le ministère de l'Agriculture et du Développement rural estime que cela réduira de 7-10\% les coûts de production supportés par les agriculteurs.

6. La mention « Toujours en cours » indique que la mesure considérée était encore en vigueur au 31 décembre 2009.

Sources:

FAO (2010), Suivi du marché du riz, divers numéros, FAO, Rome, www.fao.org/es/ESC/en/15/70/highlight 71.html.

GAIN-VM7021 (2007), Vietnam: Grain and Feed - Annual 2007, USDA/FAS, 31 mars.

GAIN-VM7057 (2007), Vietnam: Trade Policy Monitoring - Import tariffs of key agricultural and food products amended, USDA/FAS, 16 août.

GAIN-VM7082 (2007), Vietnam: FAIRS Product Specific - Import tariffs of key agricultural and food products amended, USDA/FAS, 14 novembre.

GAIN-VM8023 (2008), Vietnam: Grain and Feed - Annual 2008, USDA/FAS, 6 avril.

GAIN-VM8059 (2008), Vietnam: FAIRS Subject Report - Preferential import tariff reduction, USDA/FAS, 12 août.

GAIN-VM9022 (2009), Vietnam: Trade Policy Monitoring - Import tariffs of key agricultural and food products, USDA/FAS, 23 mars.

GAIN-VM9025 (2009), Vietnam: Grain and Feed - Annual 2009, USDA/FAS, ${ }^{\text {er }}$ avril.

Ministère de l'Agriculture et du Développement rural (2010), Vietnam agricultural news reports, consulté en janvier 2010, http://xttmnew.agroviet.gov.vn/TestE/default.asp.

Slayton, T. (2009), "Rice Price Forensics: How Asian Governments Carelessly Set the World rice Market on Fire", CGD Working Paper, n' 163, Center for Global Development, Washington, DC, www.cgdev.org/content/publications/detail/1421260/.

Banque d'État du Vietnam (2010), Annual Report, diverses années, www.sbv.gov.vn/en/home/index.jsp. 


\section{ANNEXE B. ÉVOLUTIONS DES PRIX DES PRODUITS DE BASE DEPUIS 2006}

Le graphique B.1 de l'annexe montre l'évolution des prix mensuels moyens sur les marchés internationaux de certains produits de base entre janvier 2006 et la mi-2009. La partie supérieure montre l'évolution des prix des quatre produits de base sur lesquels porte essentiellement cette étude ; la partie inférieure indique l'évolution des prix de certains autres produits. En général, les prix internationaux des denrées alimentaires de base, telles que les céréales, les oléagineux et les produits laitiers ont enregistré une augmentation bien plus spectaculaire que ceux des produits tropicaux, tels que le café et le cacao, ou des matières premières, tels que le coton ou le caoutchouc (FAO, 2009c).

Annexe graphique B.1. Évolutions des prix internationaux de certains produits agricoles de base depuis 2006 Indice janvier $2006=100$
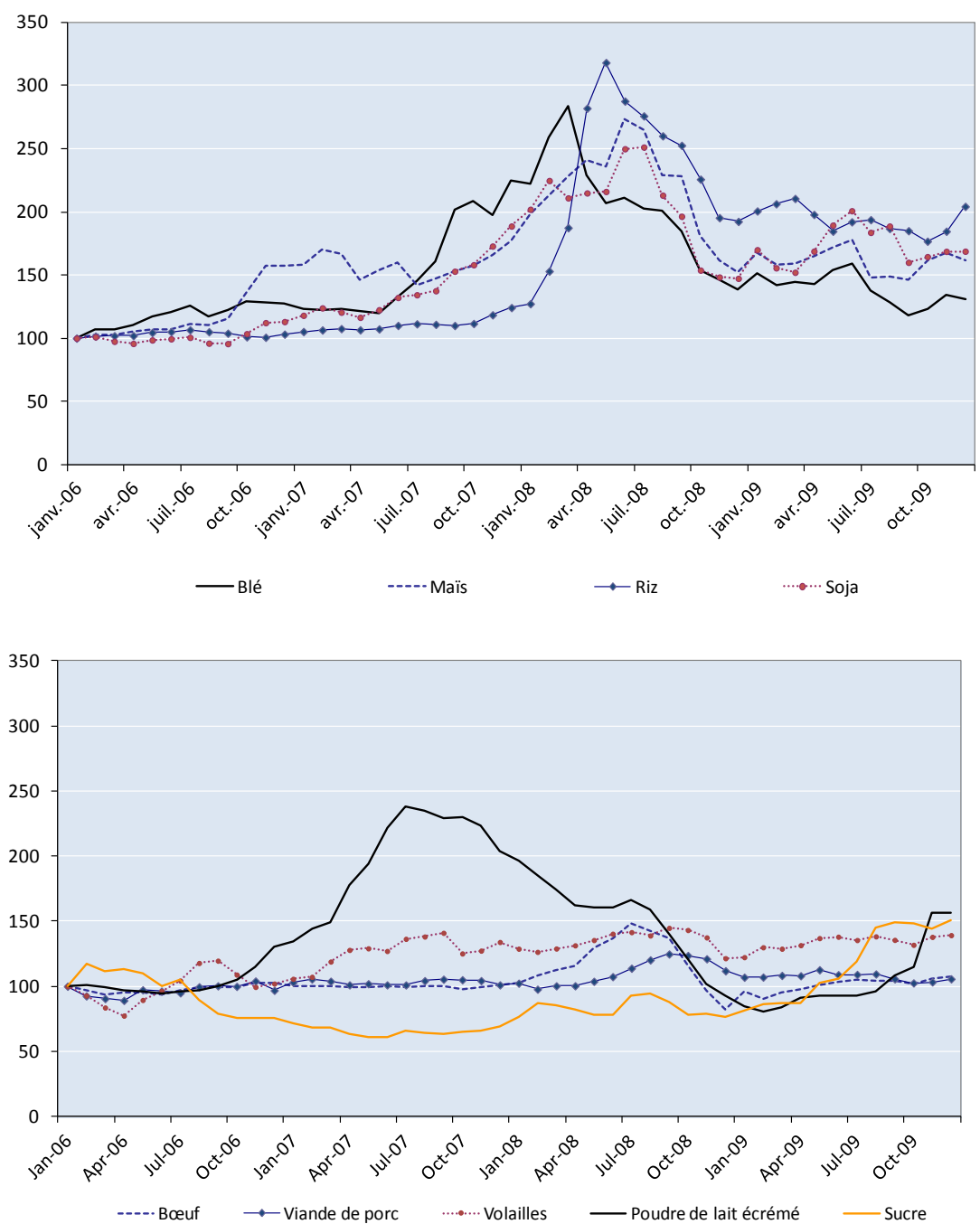

Les prix correspondent à une moyenne mensuelle.

Blé : blé dur rouge d'hiver $n^{\circ} 2$ des États-Unis, f.à.b. Golfe du Mexique

Riz : riz blanc thaïlandais de second choix $100 \% \mathrm{~B}$, f.à.b. Bangkok

Sucre : Prix journalier enregistré par l'ISA

Viande de porc : porc congelé des États-Unis, valeur unitaire à l'exportation

Poudre de lait écrémé : Océanie, prix à l'exportation indicatifs, f.à.b. l'exportation

Soja : soja jaune $n^{\circ} 1$ des États-Unis, f.à.b. Golfe du Mexique

Bœuf : viande de vache d'Australie, désossée, c.a.f. États-Unis

Volailles : Découpes de poulet des États-Unis, valeur unitaire à

Source : FAO, Prix internationaux des produits de base, 2010, www.fao.org/es/esc/prices/PricesServlet.jsp?lang=fr. 
Le prix mensuel moyen des exportations de maïs des États-Unis (maïs jaune $\mathrm{n}^{\circ} 2$ des États-Unis, f.à.b. Golfe du Mexique) a lentement augmenté, passant d'environ 100 USD la tonne en janvier 2006 (qui était également le prix annuel moyen pour 2005) à 118 USD la tonne en septembre 2006. Le prix du maïs a enregistré une forte augmentation de courte durée en octobre-novembre 2006, le prix mensuel moyen augmentant de plus d'un tiers pour atteindre 160 USD la tonne. Il est resté à ce niveau, en moyenne, pendant environ une année. Vers la fin de 2007, le prix à l'exportation du maïs a recommencé à augmenter, enregistrant une hausse de $75 \%$ en huit mois pour atteindre le niveau maximal de 291 USD la tonne en juin 2008. Au cours des six derniers mois de 2008 , le prix mensuel moyen a diminué de $44 \%$, tombant à 156 USD la tonne en décembre. Le prix à l'exportation est demeuré relativement stable autour de ce niveau tout au long de 2009. Au second trimestre 2009, le prix moyen à l'exportation était de $62 \%$ plus élevé qu'au cours de la même période en 2006, où il s'établissait à 176 USD la tonne.

Durant les neuf premiers mois de 2006, le prix mensuel moyen des exportations de soja des ÉtatsUnis (soja jaune $\mathrm{n}^{\circ} 1$, f.à.b. Golfe du Mexique) est demeuré assez stable dans une fourchette de 225 à 235 USD la tonne. Une hausse régulière des prix internationaux a commencé vers octobre 2006 et a duré pendant près de deux ans, atteignant un sommet en juillet 2008 à 586 USD la tonne, soit une augmentation de plus de $160 \%$. Faisant contraste avec cette hausse régulière, le prix international s'est effondré de $40 \%$ dans les trois mois qui ont suivi, tombant à son plus bas niveau de 344 USD par tonne en décembre. Comparativement aux trois autres produits de base, le prix international du soja a régulièrement augmenté dans la première moitié de 2009 , et au milieu de l'année il était de $90 \%$ plus élevé que le prix moyen au second trimestre 2006.

Après avoir augmenté d'environ un-tiers dans la première moitié de 2006, le prix mensuel moyen des exportations de blé des États-Unis (blé dur rouge d'hiver n ${ }^{\circ}$ 2, f.à.b. Golfe du Mexique) est demeuré assez stable pendant près d'une année, s'élevant en moyenne à 210 USD la tonne au cours de la période de 12 mois entre juin 2006 et mai 2007. À la mi-2007, le prix à l'exportation a commencé à augmenter, et au cours des neuf mois suivants il a régulièrement augmenté de près de $140 \%$ pour atteindre un niveau maximal de 482 USD la tonne en mars 2008. Le prix à l'exportation du blé a diminué presque aussi régulièrement qu'il avait augmenté, diminuant d'un peu plus de $50 \%$ en neuf mois entre mars et décembre 2008, date où il se situait à 235 USD la tonne. Le prix mensuel moyen des exportations de blé au premier trimestre 2009 était de près de 250 USD la tonne, soit de $40 \%$ plus élevé que le prix moyen au cours de la même période en 2006.

Le prix mensuel des exportations de riz thaïlandais (riz blanc de second choix $100 \% \mathrm{~B}$, f.à.b. Bangkok) a augmenté plus tardivement et plus rapidement que pour les trois autres produits de base. En 2006 et pendant la plus grande partie de 2007, le prix à l'exportation du riz a été relativement peu affecté par la hausse des prix des autres produits de base, puisqu'il a augmenté de 12\%, passant de 300 USD la tonne en janvier 2006 à un peu moins de 340 USD la tonne en octobre 2007. Dans les derniers mois de 2007, le prix à l'exportation du riz a commencé à augmenter à un rythme un peu plus rapide, avant de prendre davantage de vitesse début 2008. Il a progressé de plus de $150 \%$ dans la période de quatre mois allant de janvier à mai, date où il a atteint son niveau maximal de 963 USD la tonne. De mai à décembre 2008, le prix du riz a baissé de 40\%, tombant à 582 USD par tonne. Au cours du premier semestre 2009, le prix mensuel des exportations est demeuré relativement stable. Il était en moyenne de 580 USD la tonne au second trimestre 2009, soit 84\% de plus qu'au cours de la même période en 2006. 


\section{RÉFÉRENCES}

Abbot, P. (2009), "Development Dimensions of High Food Prices", OCDE, Alimentation, agriculture et pêcheries, Document de travail $\mathrm{n}^{\circ} 15$, OCDE, Paris, http://titania. sourceoecd.org/vl=5160982/cl=22/nw=1/rpsv/cgi-bin $/ w p p d f ? f i l e=5 \mathrm{ksfOrhn3kwb.pdf}$.

Ackah, A. et S. Appleton (2007), "Food Price Changes and Consumer Welfare in Ghana in the 1990s", Credit Research Paper No. 07/03, Centre for Research in Economic Development and International Trade, Université de Nottingham, www.nottingham.ac.uk/economics/credit/research/papers/CP0703.pdf.

Baffes, J. et B. Gardner (2003), "The Transmission of World Commodity Prices to Domestic Markets Under Policy Reforms in Developing Countries", Journal of Economic Policy Reform, Vol. 6, n 3 , septembre, pp. 159-180.

Banque mondiale (2008a), Rising Food and Fuel Prices: Addressing the Risks to Future Generations, Banque mondiale, Washington, DC, http://siteresources.worldbank.org/DEVCOMMEXT/Resources/Food-Fuel.pdf.

Banque mondiale (2008b), Framework Document for Proposed Loans, Credits and Grants in the Amount of USD 1.2 billion Equivalent for a Global Food Crisis Response Program, Banque mondiale, Washington, DC, www.escwa.un.org/rcg/documentation/12sep08/GFRPFrameworkDocument.pdf.

Banque mondiale (2008c), Rising Food Prices: Policy Options and World Bank Response, Banque mondiale, Washington, DC, http://siteresources.worldbank.org/NEWS/Resources/risingfoodprices_backgroundnote_apr08.pdf.

Banque mondiale (2008d), Rising Food Prices: The World Bank's Latin America and Caribbean Region Position Paper, Banque mondiale, Washington, DC, http://go.worldbank.org/R81KALPMS0.

Banque mondiale (2009), Global Economic Prospects 2009, Banque mondiale, Washington, DC, http://web.worldbank.org/WBSITE/EXTERNAL/EXTDEC/EXTDECPROSPECTS/GEPEXT/EXTGE P2009/0,,contentMDK:22002695 pagePK:64167689 piPK:64167673 theSitePK:5530498,00.html.

Benson, T., et al. (2008), "Global Food Crisis: Monitoring and Assessing Impact to Inform Policy Responses", Issue Brief, No. 55, IFPRI - Institut international de recherche sur les politiques alimentaires, Washington, DC, www.ifpri.org/sites/default/files/publications/pr19_1.pdf.

Brown, N., J. Laffan et M. Wright (2008), High Food Prices, Food Security and the International Trading System, Trade and Economic Analysis Branch, Department of Foreign Affairs and Trade, Canberra, paper presented to the Informal National Food Pricing Summit, www.dfat.gov.au/trade/focus/081017_food_security.html\#_edn2.

Childs, N. et J. Kiawu (2009), Factors Behind the Rise in Global Rice Prices in 2008, RCS-09D-01, rapport du Service de recherche économique (Economic Research Service - ERS), ministère de l'Agriculture des États-Unis, Washington, DC, www.ers.usda.gov/Publications/RCS/May09/RCS09D01/RCS09D01.pdf. 
CNUCED (2008), Répondre à la crise alimentaire globale : Les politiques essentielles pour le commerce, l'investissement et les produits de base afin d'assurer la sécurité alimentaire durable et d'atténuer la pauvreté, Conférence de haut niveau sur la sécurité alimentaire mondiale : les défis du changement climatique et des bioénergies, 3-5 Juin, Rome, Italie, http://www.unctad.org/fr/docs/osg20081_fr.pdf.

Dawe, D. (2008), "Have Recent Increases in International Cereal Prices been Transmitted to Domestic Economies? The Experience in Seven Large Asian Countries", ESA Working Paper, n 08-03, avril, Division de l'économie du développement agricole, FAO, Rome, ftp://ftp.fao.org/docrep/fao/010/ai506e/ai506e00.pdf.

De Gregorio, J. (2008), "Monetary Policy and Commodity Prices in Turbulent Times", 2008 IIF Annual Meeting of Latin American Chief Executives, Santiago, Chili, www.bcentral.cl/jdegrego/pdf/jdg02042008.pdf.

Demeke, M., G. Pangrazio et M. Maetz (2008), Country Responses to the Food Security Crisis: Nature and Preliminary Implications of the Policies Pursued, FAO, Rome, www.fao.org/fileadmin/user_upload/ISFP/pdf_for_site_Country_Response_to_the_Food_Security.p df.

Dessus, S., S. Herrera et R. de Hoyos (2008), "The Impact of Food Inflation on Urban Poverty and its Monetary Cost: Some Back-of-the-Envelope Calculations", Policy Research Working Paper 4666, Banque mondiale, Washington, DC, http://econ.worldbank.org.

Dewbre, J. et al. (2008), "High Food Commodity Prices: Will They Stay? Who Will Pay?", Agricultural Economics, Vol. 39, Supplement, pp. 393-403.

FAO (2008a), La flambée des prix des denrées alimentaires: faits, perspectives, effets et actions requises, HLC/08/INF/1, Conférence de haut niveau sur la sécurité alimentaire mondiale : les défis du changement climatique et des bioénergies, 3-5 juin, FAO, Rome, http://www.fao.org/fileadmin/user upload/foodclimate/HLCdocs/HLC08-inf-1-F.pdf.

FAO (2008b), "National Policy Responses to High Food Prices", Economic and Social Perspectives Policy Brief 1, juillet, FAO, Rome, ftp://ftp.fao.org/docrep/fao/010/aj113e/aj113e00.pdf.

FAO (2008c), Policy Measures Taken by Governments to Reduce the Impact of Soaring Food Prices (as of 15 December 2008), Système mondial d'information et d'alerte rapide sur l'alimentation et l'agriculture (SMIAR), FAO, Rome, www.fao.org/giews/english/policy/index.asp.

FAO (2009a), Mesures prises face à la hausse des prix des denrées alimentaires, CCP 09/8, Comité des produits, Soixante-septième session, 20-22 avril, FAO, Rome.

FAO (2009b), Conséquences macroéconomiques et gestion des fluctuations des prix des produits alimentaires, CCP 09/10, Comité des produits, Soixante-septième session, 20-22 avril, FAO, Rome ftp://ftp.fao.org/docrep/fao/meeting/016/k4223f.pdf.

FAO (2009c), The State of Agricultural Commodity Markets 2009, FAO, Rome, www.fao.org/docrep/012/i0854e/i0854e00.htm.

Fonds monétaire international [FMI] (2008a), Food and Fuel Prices - Recent Developments, Macroeconomic Impact and Policy Responses, prepared by the Fiscal Affairs, Policy Development and Review and Research Departments, 30 juin, FMI, Washington, DC, www.imf.org/external/np/pp/fre/2008/063008.pdf. 
FMI (2008b), Food and Fuel Prices - Recent Developments, Macroeconomic Impact and Policy Responses: An Update, prepared by the Fiscal Affairs, Policy Development and Review and Research Departments, 19 septembre, FMI, Washington, DC, www.imf.org/external/np/pp/eng/2008/091908.pdf.

Grosh, M., et al. (2008), Pour la protection et la promotion : Conception et mise en cuvre de filets sociaux de sécurité efficaces, Banque mondiale, Washington, DC, http://siteresources.worldbank.org/SAFETYNETSANDTRANSFERS/Resources/2819451223052223982/ProtectionandPromotion-Overview-Fr.pdf.

James, W., et al. (2008), Food prices and Inflation in Developing Asia: Is Poverty Reduction Coming to an End?, Special report Economics and Research Department, Banque asiatique de développement, Manille, www.adb.org/Documents/reports/food-prices-inflation/food-prices-inflation.pdf.

Janvry, A. de et E. Sadoulet (2008), "The Global Food Crisis: Identification of the Vulnerable and Policy Responses", Agricultural Resource Economics Update, Vol. 12, n² 2, nov/déc, Giannini Foundation of Agricultural Economics, Université de Californie, www.agecon.ucdavis.edu/extension/update/articles/v12n2_7.pdf.

Leyaro, V. (2009), "Commodity Price Changes and Consumer Welfare in Tanzania in the 1990s and 2000s", document présenté à la Conférence de 2009 du Centre d'études sur les économies africaines (CSAE) sur le développement économique en Afrique, St. Catherine's College, Oxford, www.csae.ox.ac.uk/conferences/2009-EDiA/papers/345-Leyaro.pdf.

Ligon, E. (2008), "Food Prices and the Welfare of Poor Consumers", Agricultural Resource Economics Update, Vol. 12, n 2, nov/déc, Giannini Foundation of Agricultural Economics, University of California, www.agecon.ucdavis.edu/extension/update/articles/v12n2_6.pdf.

Lindert, K., et al. (2007), "The Nuts and Bolts of Brazil’s Bolsa Família Program: Implementing Conditional Cash Transfers in a Decentralized Context", SP Discussion Paper $n^{\circ}$ 0709, Banque mondiale, www.ipc-undp.org/publications/cct/brazil/BRBolsaFamiliaDiscussionPaper.pdf.

Lustig, N. (2009), "Coping with Rising Food Prices: Policy Dilemmas in the Developing World”, CGD Working Paper $n^{\circ}$ 164, Center for Global Development, Washington, DC, www.cgdev.org/content/publications/detaile/1421334/.

OCDE (2008), Estimation du soutien aux producteurs et autres indicateurs du soutien à l'agriculture élaborés par l'OCDE : concepts, calculs, interprétation et utilisation (Manuel sur l'ESP), Direction des échanges et de l'agriculture, OCDE

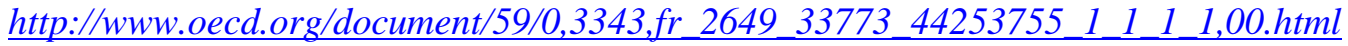

OCDE (2009a), Politiques agricoles des économies émergentes 2009 : Suivi et évaluation, OCDE, Paris.

OCDE (2009b), Forum mondial sur l'agriculture- Perspectives agricoles : préparer l'avenir, OCDE, Paris, www.oecd.org/agriculture/globalforum/june09.

OCDE (2009c), Perspectives agricoles de l'OCDE et de la FAO 2009-2018, OCDE, Paris.

Programme alimentaire mondial [PAM] (2009), Collection « La faim dans le monde », La faim et le rôle des marchés, Publiée par Earthscan, Londres, http://documents.wfp.org/stellent/groups/public/documents/newsroom/wfp209118.pdf.

Rapsomanikis, G., D. Hallam et P. Conforti (2004), "Intégration des marchés et transmission des prix pour certains marchés de cultures vivrières et commerciales de pays en développement : analyse et applications", Rapport sur les marchés des produits 2003-2004, FAO, Rome, pp. 55-82, http://www.fao.org/docrep/007/y5117f/y5117foo.htm. 
Seale, J. Jr., A. Regmi et J. Bernstein (2003), "International Evidence on Food Consumption Patterns", Technical Bulletin $n^{\circ} T B-1904$, ERS, USDA, Washington, DC, www.ers.usda.gov/publications/tb1904/.

Sharma, R. (2003), "The Transmission of World Price Signals: The Concept, Issues and Some Evidence from Asian Cereal Markets", Agricultural Trade and Poverty: Making Policy Analysis Count, OCDE, Paris, pp. 141-160.

Slayton, T. (2009), "Rice Price Forensics: How Asian Governments Carelessly Set the World rice Market on Fire", CGD Working Paper, ${ }^{\circ}$ 163, Center for Global Development, Washington, DC, www.cgdev.org/content/publications/detail/1421260/.

Thapa, G., et al. (2009), "Soaring Food Prices: A Threat or Opportunity in Asia?", BWPI Working Paper, $\mathrm{n}^{\circ}$ 69, Brookings World Poverty Institute, Université de Manchester, Manchester, www.bwpi.manchester.ac.uk/resources/Working-Papers/bwpi-wp-6909.pdf.

Thompson W. et G. Tallard (2010), "Effets potentiels sur les marchés de certaines mesures envisageables dans les économies émergentes pour parer aux futures flambées des prix des produits agricoles de base", OCDE, Alimentation, agriculture et pêcheries, à paraitre, OCDE, Paris.

Timmer, P. (2008), "Causes of High Food Prices", ADB Economic Working Paper Series, $\mathrm{n}^{\circ} 128$, octobre, Banque asiatique de développement, Manille, www.adb.org/Documents/WorkingPapers/2008/Economics-WP128.pdf.

Timmer, P. (2009), “Did Speculation Affect World Rice Prices?”, ESA Working Paper, n 09-07, avril, Division de l'économie du développement agricole, Organisation des Nations Unies pour l'alimentation et l'agriculture, ftp://ftp.fao.org/docrep/fao/011/ak232e/ak232e00.pdf.

UkrAgroConsult (2009), The Public and Private Sectors in the Sustainable Development of the Ukraine Grain Sector, document rédigé pour une table ronde organisée par la FAO et la Banque Européenne pour la Reconstruction et le Développement (BERD) qui s'est tenue le 22 avril, www.eastagri.org/meetings/index.asp? id =40.

Viatte, G., et al. (2009), Responding to the Food Crisis: Synthesis of Medium-Term Measures Proposed in Inter-Agency Assessments, FAO, Rome, www.fao.org/fileadmin/user_upload/ISFP/SR_Web.pdf.

Yu, Tun-Hsiang, et al. (2009). "A Quantitative Analysis of Trade Policy Responses to High Agricultural Commodity Prices", contribution à la Conférence de l'Association internationale des économistes agricoles, 16-22 août, Pékin, Chine, http://ageconsearch.umn.edu/bitstream/51805/2/IAAE 734.pdf.

Zezza, A., et al. (2009), "The Impact of Rising Food Prices on the Poor”, contribution à la Conférence de l'Association internationale des économistes agricoles, 16-22 août, Pékin, Chine, http://ageconsearch.umn.edu/bitstream/51696/2/Zezza\%20et\%20al\%20IAAE-2.pdf. 